\title{
THE USE OF WATER VAPOR AS A REFRIGERANT: IMPACT OF CYCLE MODIFICATIONS ON COMMERCIAL FEASIBILITY
}

Final Report

Date Published - August 2004

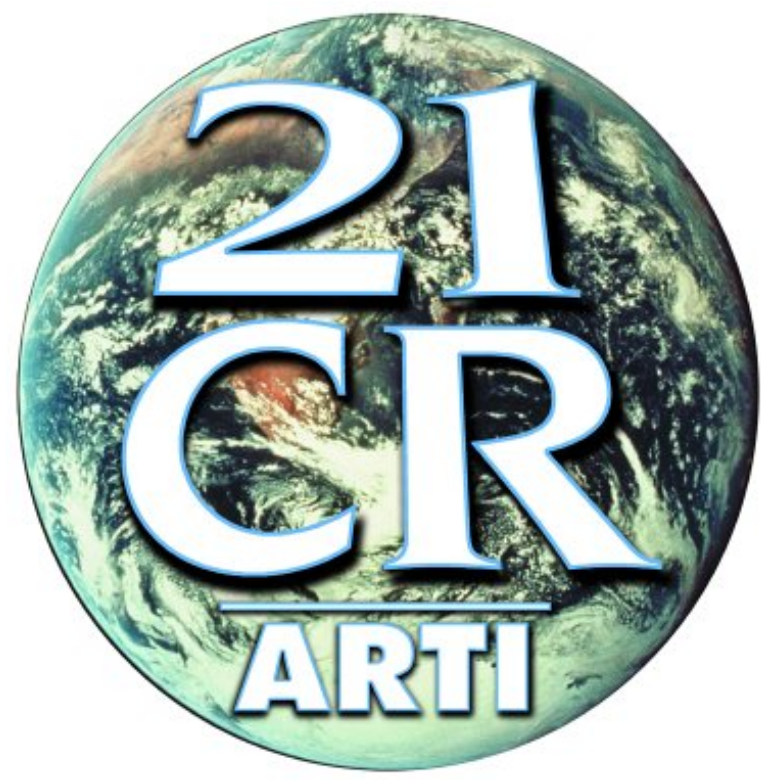

Authors: Brandon F. Lachner Jr, Gregory F. Nellis, and Douglas T. Reindl

UNIVERSITY OF WISCONSIN SOLAR ENERGY LABORATORY

1500 Engineering Drive, Madison, WI 53706

Prepared for the

AIR-CONDITIONING AND REFRIGERATION TECHNOLOGY INSTITUTE

4100 N. Fairfax Drive, Suite 200, Arlington, Virginia 22203

Distribution A - Approved for public release; further dissemination unlimited. 


\section{DISCLAIMER}

This report was prepared as an account of work sponsored by the Air-Conditioning and Refrigeration Technology Institute (ARTI) under its "HVAC\&R Research for the $21^{\text {st }}$ Century" (21CR) program. Neither ARTI, the financial supporters of the 21CR program, or any agency thereof, nor any of their employees, contractors, subcontractors or employees thereof - makes any warranty, expressed or implied; assumes any legal liability or responsibility for the accuracy, completeness, any third party's use of, or the results of such use of any information, apparatus, product, or process disclosed in this report; or represents that its use would not infringe privately owned rights. Reference herein to any specific commercial product, process, or service by trade name, trademark, manufacturer, or otherwise, does not necessarily constitute nor imply its endorsement, recommendation, or favoring by ARTI, its sponsors, or any agency thereof or their contractors or subcontractors. The views and opinions of authors expressed herein do not necessarily state or reflect those of ARTI, the $21 \mathrm{CR}$ program sponsors, or any agency thereof.

Funding for the 21CR program provided by (listed in order of support magnitude):

- U.S. Department of Energy (DOE Cooperative Agreement No. DE-FC05-99OR22674)

- Air-Conditioning \& Refrigeration Institute (ARI)

- Copper Development Association (CDA)

- New York State Energy Research and Development Authority (NYSERDA)

- California Energy Commission (CEC)

- Refrigeration Service Engineers Society (RSES)

- Heating, Refrigeration and Air Conditioning Institute of Canada (HRAI)

Available to the public from

U.S. Department of Commerce

National Technical Information Service

5285 Port Royal Road

Springfield, VA 22161

(703) 487-4650

Available to U.S. Department of Energy and its contractors in paper from

U.S. Department of Energy

Office of Scientific and Technical Information

P.O. Box 62

Oak Ridge, TN 37831

(423) 576-8401 


\title{
THE USE OF WATER VAPOR AS A REFRIGERANT: IMPACT OF CYCLE MODIFICATIONS ON COMMERCIAL FEASIBILITY
}

\author{
Final Report
}

Date Published - August 2004

Authors: Brandon F. Lachner, Jr., Gregory F. Nellis, and Douglas T. Reindl

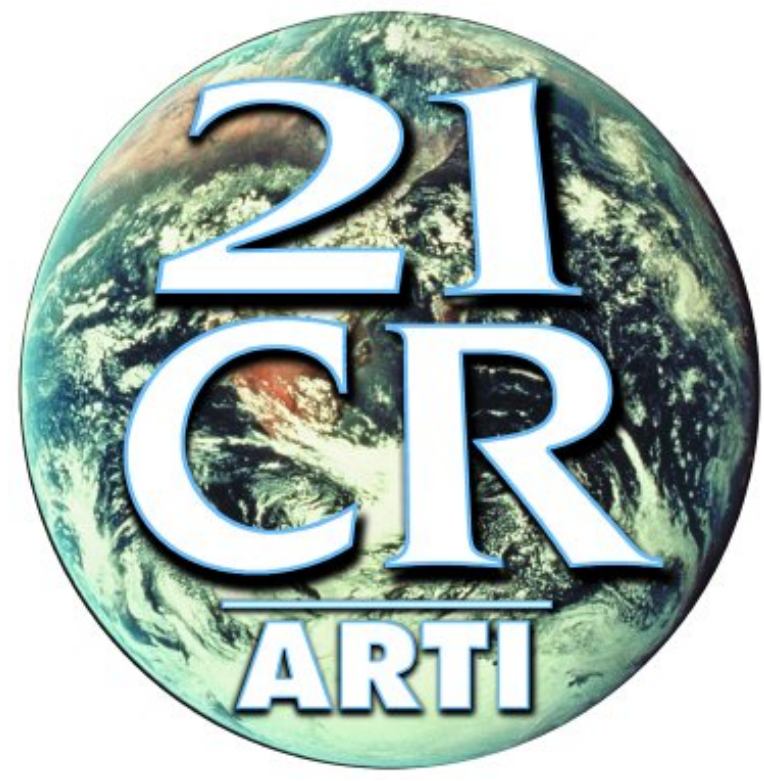

Prepared for the

AIR-CONDITIONING AND REFRIGERATION TECHNOLOGY INSTITUTE

Under ARTI 21CR Program Contract Number 611-10080 



\section{EXECUTIVE SUMMARY}

\section{THE USE OF WATER VAPOR AS A REFRIGERANT: IMPACT OF CYCLE MODIFICATIONS ON COMMERCIAL FEASIBILITY}

The use of water as a refrigerant in a vapor compression chiller does have several attractive attributes. Water is environmentally friendly, low cost, and non-toxic. The thermophysical properties of water are consistent with a vapor compression chiller system that has the potential to achieve a high coefficient of performance (COP). The possibility of using water as both the primary refrigerant and secondary system working fluid offers the potential to implement direct-contact heat exchangers. Direct-contact heat exchangers are both less expensive and can yield improved performance over their indirect heat exchanger counterparts.

Along with the above-mentioned advantages, a water-based vapor compression chiller does have several challenging characteristics. As a point of reference, Table 1 shows that water can compete with R-134a, in terms of its theoretical COP; however, Table 1 also highlights some of the key differences between water (R-718) and R-134a operating between conditions (i.e. saturation temperatures) typically encountered in large tonnage chillers. The major differences are related to cycle parameters such as peak temperature, pressure ratio, operating pressures, and volumetric flow rates.

Figure 1 shows a pressure-volume diagram for the ideal R-134a and water vapor compression cycles. This figure illustrates the several problems with a water-based vapor compression cycle that must be addressed. One of the challenges lies in the compression process itself. Water has an extremely high specific volume at normal suction conditions for chiller applications and also requires a large compression ratio to reject heat from the 
cycle. The superheat loss incurred in the water cycle is nearly two orders of magnitude larger than in the R-134a cycle. On the other hand, Table 1 indicates that throttling loss in a water cycle is relatively low so that the benefit associated with any work extracting expansion device is negligible.

The combination of the large superheat loss and pressure ratio requirements suggest that a multi-stage compression cycle with intercooling may be the most practical and economically-viable alternative. Therefore, various cycle configurations with different methods of intercooling were screened using low-level models. The most attractive cycle configuration is modeled in more detail with component-level models, as described in the second section of the paper. Detailed component models are necessary in order to realistically assess performance and develop relationships between component sizing alternatives and life-cycle costs. A similar analysis of a more conventional R-134a cycle is carried out to provide a meaningful basis of comparison.

Table 1 Characteristics of water v. R-134a refrigeration cycle

\begin{tabular}{|c|c|c|}
\hline Quantity & Water & R-134a \\
\hline Theoretical coefficient of performance, COP & 8.39 & 8.47 \\
\hline Carnot Coefficient of Performance, $\mathrm{COP}_{\text {Carnot }}$ & \multicolumn{2}{|c|}{9.88} \\
\hline $\mathrm{COP} / \mathrm{COP}_{\text {Carnot }}$ & 0.85 & 0.86 \\
\hline Compressor discharge temperature, $\mathrm{T}_{2 \mathrm{~s}}$ & $313^{\circ} \mathrm{F}$ & $101^{\circ} \mathrm{F}$ \\
\hline Throttling loss, $\left(\mathrm{s}_{4}-\mathrm{S}_{3}\right) / \mathrm{Q}, \mathrm{R}^{-1}$ & $4.7 \mathrm{e}-6$ & $3.0 \mathrm{e}-5$ \\
\hline Superheat loss, $\mathrm{S}_{\text {gen,sh }} / \mathrm{Q}^{1}, \mathrm{R}^{-1}$ & $2.83 \mathrm{e}-4$ & $9.0 \mathrm{e}-6$ \\
\hline Compressor inlet specific volume, $\mathrm{v}_{1}, \mathrm{ft}^{3} / \mathrm{lbm}$ & 2111 & 0.88 \\
\hline Compressor suction pressure, $\mathrm{p}_{1}, \mathrm{psia}$ & 0.14 & 54 \\
\hline Compressor saturated suction temperature, $T_{1, \text { sat }}$ & \multicolumn{2}{|c|}{$44^{\circ} \mathrm{F}$} \\
\hline Compressor discharge pressure, $\mathrm{p}_{2}$, psia & 0.82 & 129 \\
\hline Compressor saturated discharge temperature, $T_{2, \text { sat }}$ & \multicolumn{2}{|c|}{$95^{\circ} \mathrm{F}$} \\
\hline Compression ratio, $\mathrm{PR}$ & 5.75 & 2.4 \\
\hline Pressure lift, $\mathrm{p}_{2}-\mathrm{p}_{1}, \mathrm{psia}$ & 0.7 & 75 \\
\hline Compressor inlet volumetric flow rate, $\mathrm{ft}^{3} / \mathrm{s}$ & 6900 & 45 \\
\hline Isentropic enthalpy difference across compressor, $\Delta \mathrm{h}_{\mathrm{s}}$, & 121 & 7.8 \\
\hline
\end{tabular}

1. $\mathrm{S}_{\mathrm{gen}, \mathrm{sh}}$ is the entropy generated by the irreversible de-superheat process 


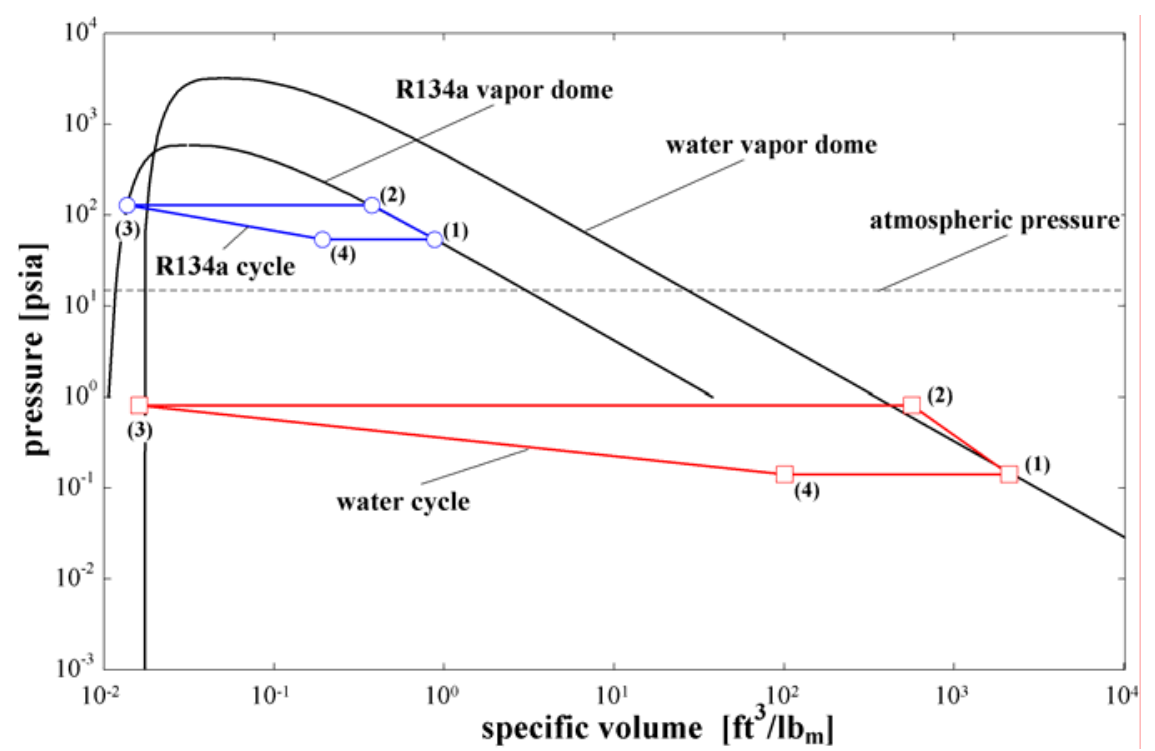

Figure 1 Pressure-specific volume diagrams of ideal water and R-134a vapor compression refrigeration cycles.

\section{CYCLE SELECTION}

Multi-stage compression will be required for a water as refrigerant cycle due to the large pressure ratio and high volumetric flow rate requirements. Therefore, the implementation of intercooling may not add significantly to cycle complexity but does provide substantial gains in system COP, as evidenced by Table 1. Therefore, the focus of the cycle selection study was on comparing a non-intercooled cycle with a number of different cycle configurations capable of providing intercooling, including: economized cycle, flash intercooled cycle, direct intercooled cycle, and indirect intercooled cycle. A liquid subcooled cycle was also investigated. These cycle configurations are compared against the non-intercooled, two-stage compression cycle shown in Figure 6. A schematic of some of these cycle configurations are shown in Figures 2 through 5. All of the cycles are modeled using the Engineering Equation Solver (EES) software (Klein and Alvarado, 2004). Component modeling for these screening models consists of single- 
value performance metrics including a polytropic compression efficiency ${ }^{1}$ (assumed to be $70 \%$ ) and heat exchanger approach temperatures (assumed to be $0^{\circ} \mathrm{C}$ for the evaporator and $0.5^{\circ} \mathrm{C}$ for the condenser). The use of a polytropic rather than isentropic compression efficiency ensures that the overall performance of the compression process was not influenced by the number of compression stages; thereby masking true changes in the system performance related to intercooling between stages.

Figure 6 illustrates the resulting COP for several of the cycle configurations as a function of the number of compression stages. Also shown in Figure 6 is the COP of a non-intercooled R-134a cycle modeled using the same performance metrics. The economized configuration shown in Figure 3 works well in an R-134a cycle but provides very little intercooling in a water cycle. In an economized cycle, the two-phase fluid leaving the expansion valve is separated by phase: the vapor is mixed with the compressor exit stream (superheated steam) in order to provide some cooling while the intermediate pressure/temperature liquid is passed on to the next expansion valve in series. Because the throttling loss in the water cycle is so small (see Table 1), the amount of flash gas produced by each expansion is small and therefore there is very little intercooling realized between stages.

Flash intercooling (Figure 4) uses the direct injection (e.g. via spray) of liquid water into the superheated vapor produced between compression stages. Liquid water from the condenser exit is expanded into the compressor discharge so that the heat of

\footnotetext{
${ }^{1}$ The polytropic efficiency is defined as being equal to the isentropic efficiency at an infinitesimally small pressure ratio. When applied to a finite pressure ratio, a compressor with a given polytropic efficiency will have a lower isentropic efficiency.
} 
compression is absorbed by the process of vaporizing the throttled liquid. The benefit of this cycle configuration is that the temperature of the gas between stages can be reduced dramatically, in theory to the saturation temperature. The drawback of the flash intercooled cycle is that the mass flow rate passing through subsequent compressor stages increases; however, Figure 6 illustrates that the COP of the flash intercooled cycle is significantly improved relative to the non-intercooled cycle. The degree of improvement diminishes with the number of compression stages but a large increase is seen with even a two-stage compression process.

In an indirect intercooled cycle (Figure 5), the intercooling is provided using an indirect contact heat exchanger that cools the intermediate pressure vapor using the condenser cooling water. The drawback of this cycle configuration is that the temperature of the vapor entering the subsequent compressor stage can only be cooled to the condenser supply water temperature plus some approach temperature. Also, a significant amount of condenser water must be used because it is not being evaporated, as in the flash intercooled cycle, and therefore the heat rejection results only in a sensible energy gain. The benefit of this cycle configuration is that the downstream compressors do not have to process additional refrigerant mass as in the flash intercooled cycle. Figure 6 shows that these two effects approximately cancel so that the COP of the indirect intercooled cycle is nearly the same as that of the flash intercooled cycle. In a water cycle, the use of the indirect intercooler presents some significant practical difficulties including added pressure loss between compression stages as well as additional capital costs for the heat exchangers. Although pressure drop is neglected in this simplified analysis, more detailed models show that the pressure drop is a driving 
consideration for system design due to the combination of large volumetric flow rates and low absolute cycle pressures and pressure lift.

The direct intercooled and liquid subcooled cycles were found to be impractical for a water cycle and therefore the performance of these cycles are not presented in Figure 6. Any significant amount of standing water (>1") is associated with a hydrostatic pressure rise that is large relative to the overall pressure lift for the cycle. Therefore, the COP penalty associated with standing water eliminates direct and liquid subcooled cycles from consideration.

The baseline configuration selected for this project is the flash intercooled cycle. This cycle is analyzed in more detail by integrating the component-level models, described in the next subsection, with the thermodynamic system models in order to allow an economic analysis to be carried out, described in the following subsection. Issues that are not captured by this analysis but still important when considering water as a refrigerant are discussed at the end. 


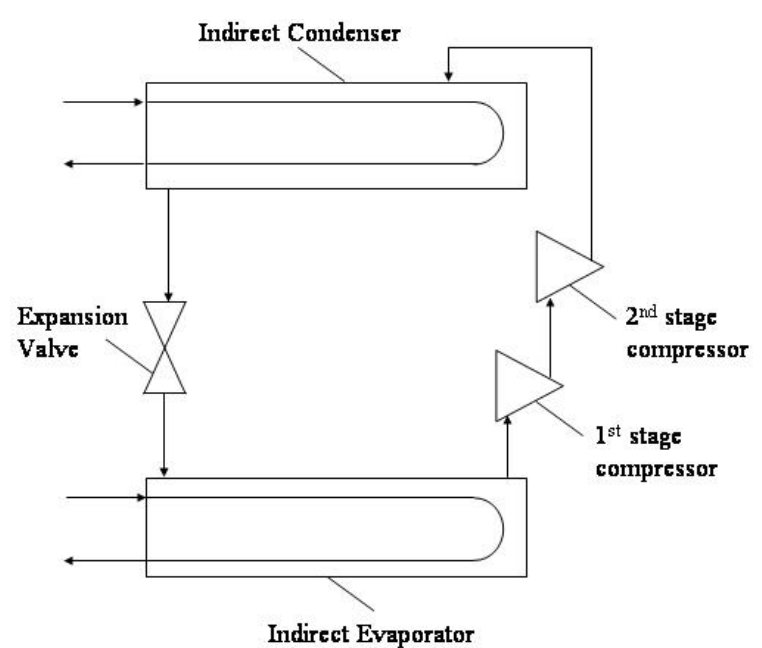

Figure 2. Non-intercooled cycle

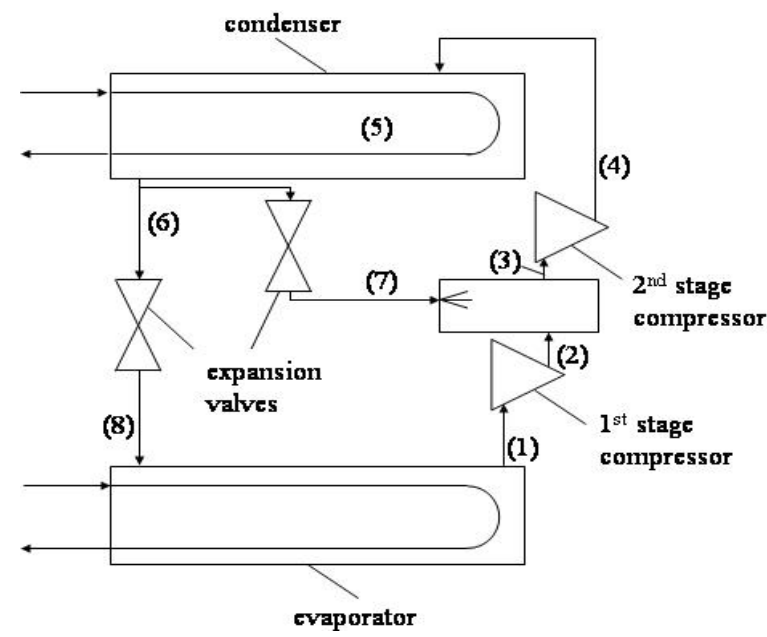

Figure 4. Flash intercooled cycle

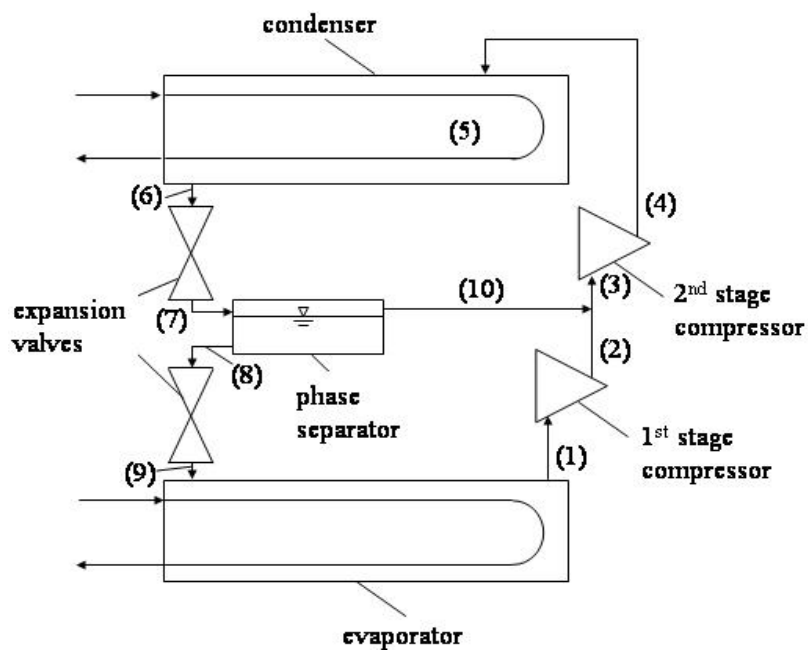

Figure 3. Economized cycle

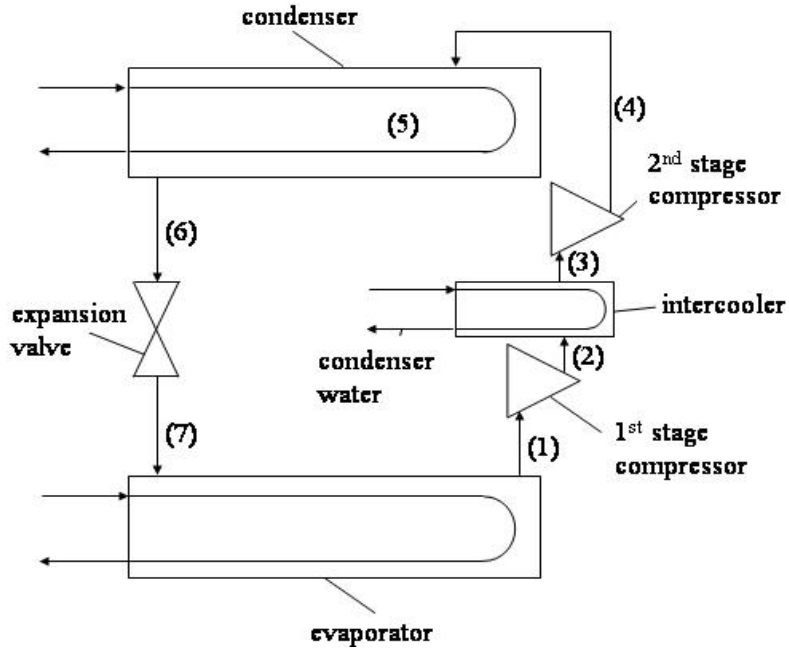

Figure 5. Indirect intercooled cycle

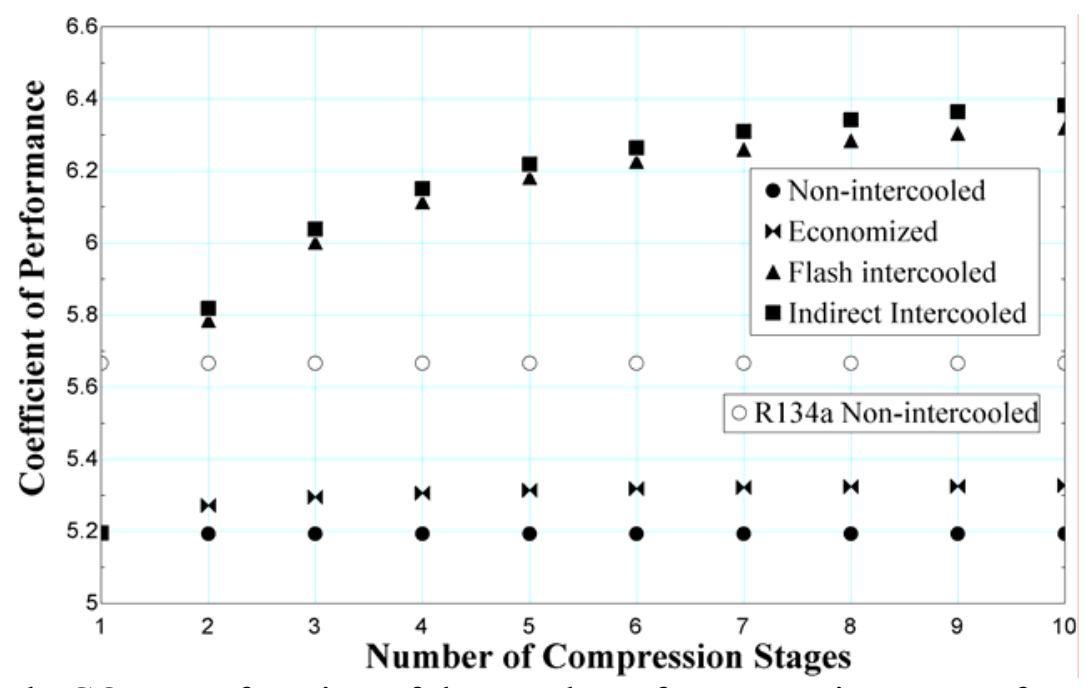

Figure 6 Cycle COP as a function of the number of compression stages for a single stage R-134a cycle and two-stage water cycles that include: non-intercooled, economized, flash intercooled, and indirect intercooled. 


\section{COMPONENT MODELING}

Component models are developed to provide more detailed sizing and performance estimates for the components integrated into a chiller system.

\section{Compressor}

Wight et al. (2000) carried out a detailed scoping analysis of turbo-compressor technology applied to steam compression in a water as a refrigerant cycle. The authors concluded that a single-stage compressor was not feasible for several reasons including: low efficiency (less than $75 \%$ for the radial bladed unit), very large impellers (ca. $20 \mathrm{ft}$ in diameter), and very high tip velocities (ca. $2200 \mathrm{ft} / \mathrm{sec}$ ). The high tip velocities lead to large stresses and high Mach numbers. These factors combine to yield extremely high capital cost and technically-challenging compressor design/development. A two stage centrifugal compressor was also evaluated with improved results. The two-stage configuration was able to achieve predicted efficiencies of $80 \%$ with large impeller diameters but with somewhat lower tip velocities (nominally $1600 \mathrm{ft} / \mathrm{sec}$ ) implying a more technically achievable compressor. The most promising compressor technology identified by Wight et al., in terms of total geometric size and performance, was a multistage axial compressor configuration consisting of between $6 \& 7$ stages. The efficiency of this type of compressor was estimated at $82 \%$ and the size and tip speeds required were reasonable, $4.5 \mathrm{ft}$ and $1400 \mathrm{ft} / \mathrm{sec}$, respectively. The disadvantage is that axial compressors are sophisticated and expensive devices when compared to centrifugal compressors. Therefore, the use of axial compressors is generally limited to high unit cost devices such as jet engines and stationary gas turbines for power production. In contrast, centrifugal compressors are sufficiently inexpensive to allow their use in very 
large capacity refrigeration systems today. Given the inherently high cost of axial compression systems, it seems likely that a multi-stage compression process using centrifugal compressors will be more economically attractive and therefore this technology became the focus of the compressor modeling carried out in this project.

The centrifugal compressor is modeled as a series of thermomechanical processes that the refrigerant transitions through corresponding to the inlet nozzle, impeller, vaneless space, vaned diffuser, and collector. Simple relations are used to establish the performance of each sub-component. Taken together, these sub-component models make up the centrifugal compressor model which is capable of predicting the static and stagnation states of the refrigerant as it moves along a mean-line path between the compressor inlet and the exit. The static and stagnation state points are illustrated graphically in Figure 7 on a T-s diagram (for a steam compression process). The compressor model is used to estimate the performance, size, and cost of both water vapor compressors and R-134a compressors.

(1)

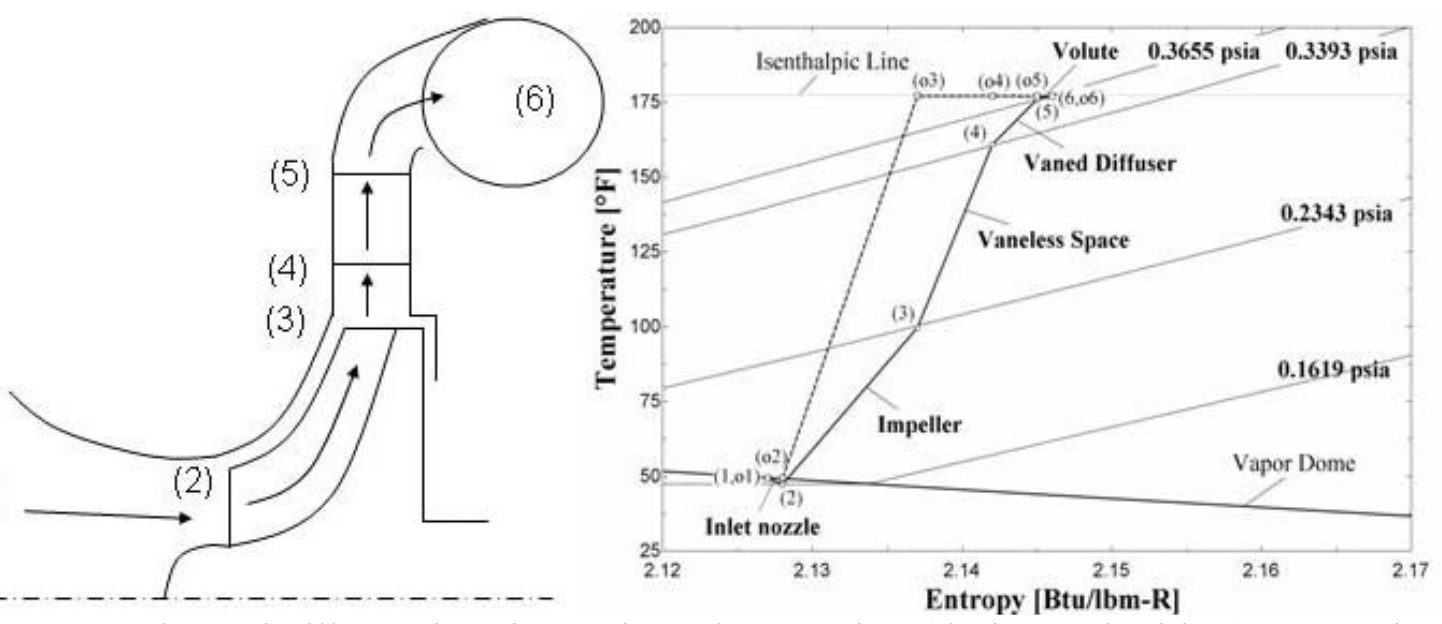

Figure 7 Schematic illustrating the static and stagnation (designated with o) state points within a compressor alongside the corresponding T-s diagram (for a water compression process). 


\section{Heat Exchangers}

The heat exchanger models rely on establishing a given set of geometric and other relationships (i.e. aspect ratio, tube pitch, approach temperature difference, refrigerant velocity) that can be used to develop performance predictions which lead to specific heat exchanger sizes. Both the direct and indirect-contact heat exchangers function similarly in this respect. Based on the discussion above regarding the effect of standing water on cycle performance, heat transfer coefficients for water as refrigerant cycles are based on correlations for falling film evaporation and condensation over tube-banks. The R-134a baseline cycle is assumed to use a flooded evaporator. These heat transfer coefficients are all based on correlations for smooth tubes found in Rohsenow et al. (1998). The Petukhov equation (Incropera and DeWitt, 2002) for internal pipe flow is used on the water side. These relationships allow the overall conductance to be calculated and the heat exchanger geometry is iterated until the calculated conductance meets performance requirements. Conductance values were corrected using a multiplier that accounts for the effect of enhanced surfaces in order to match performance data obtained from several chiller manufacturers for an R-134a chiller in the 1000-ton size range.

The direct contact heat exchanger is assumed to be a shell filled with a packing material having a high specific surface area in order to increase the wetted heat transfer area. The direct contact heat exchanger model predicts the volume of packing material that is required to provide adequate heat transfer surface area given the cycle requirements. Heat transfer coefficients are calculated based on hydrodynamic conditions which are heavily dependent on the allowable refrigerant pressure drop (velocity) through the shell. 
In the water cycle pressure drop becomes a key issue, almost as important as heat transfer surface area. The flexibility of the indirect-contact heat exchanger models to adjust geometric levers in order to control the pressure drop is critical to optimizing the water cycle. Tube pitch and refrigerant velocity are the main levers for controlling pressure drop. Pressure drop in the R-134a cycle is a negligible penalty, thereby making tube pitch a minor concern.

\section{ECONOMIC ANALYSIS}

The viability of a water-based vapor compression refrigeration system revolves around its life-cycle cost competitiveness with current technology. The life-cycle cost of investing in either an R-134a cycle or a water cycle are evaluated using a multiplier method known as the " $\mathrm{P}_{1}-\mathrm{P}_{2}$ " approach (Duffie and Beckman, 1991). The parameter, $\mathrm{P}_{1}$ is a multiplier that establishes the present value of future energy costs. This essentially modifies the operating cost so that it can be compared directly (i.e. in present value) to the capital cost. The parameter, $\mathrm{P}_{2}$, is a multiplier that accounts for all of the economic parameters that pertain to the capital cost of the equipment. This multiplier varies depending on parameters such as the proportion of the capital cost that is financed, tax rates, inflation rates, etc. Using these multipliers, the life-cycle cost (LCC) is defined by the following equation in real (today's) dollars.

$$
\mathrm{LCC}=\mathrm{P}_{1} \cdot \mathrm{C}_{\mathrm{O}}+\mathrm{P}_{2} \cdot \mathrm{C}_{\mathrm{F}}
$$

where $\mathrm{C}_{\mathrm{O}}$ is the yearly operating cost and $\mathrm{C}_{\mathrm{F}}$ is the capital cost. In order for a water as a refrigerant cycle to be a viable economic alternative to more traditional synthetic refrigerant-based cycles, any capital cost premium that is required for the water refrigeration cycle equipment (e.g., the large centrifugal compressors) must be balanced 
by an operating cost saving associated with the most efficient water cycle configurations. Although R-134a has a slightly higher theoretical COP, the water cycle configuration chosen had a high efficiency due to aerodynamic constraints. With this in mind, the maximum allowable first cost is calculated by setting the life-cycle costs of the two cycles, the baseline R-134a cycle and an alternative water cycle, equal to one another in the following way.

$$
\mathrm{C}_{\mathrm{F}, \text { water }}=\frac{\mathrm{P}_{1} \cdot \mathrm{C}_{\mathrm{O}, \mathrm{R} 134 \mathrm{a}}+\mathrm{P}_{2} \cdot \mathrm{C}_{\mathrm{F}, \mathrm{R} 134 \mathrm{a}}-\mathrm{P}_{1} \cdot \mathrm{C}_{\mathrm{O} \text {,water }}}{\mathrm{P}_{2}}
$$

where $\mathrm{C}_{\mathrm{F} \text {,water }}$ is the maximum allowable first cost of a competing water cycle, $\mathrm{C}_{\mathrm{O}, \mathrm{R} 134 \mathrm{a}}$ is the first cost for the R-134a cycle (obtained via a survey of chiller manufacturers), and $\mathrm{C}_{\mathrm{O} \text {,water }}$ and $\mathrm{C}_{\mathrm{O}, \mathrm{R} 134 \mathrm{a}}$ are the yearly operating costs associated with a water and $\mathrm{R}-134 \mathrm{a}$ cycle, respectively. For the four possible permutations of the flash intercooled water cycle considered, Table 2 summarizes the results of a 20 -year life cycle analysis. Table 2 shows both the maximum allowable and the estimated first cost of each water cycle permutation. The permutations correspond to the various combinations of direct and indirect heat exchangers that are possible.

Table 2 Results of 20-year life-cycle cost analysis.

\begin{tabular}{l|c|c} 
Cycle Configuration & $\begin{array}{c}\text { Maximum Allowable } \\
\text { First Cost }\end{array}$ & $\begin{array}{c}\text { Predicted Actual } \\
\text { First Cost }\end{array}$ \\
\hline Indirect Condenser and Evaporator & $\$ 83,700$ & $\$ 1,098,000$ \\
\hline Indirect Condenser / Direct Evaporator & 83,900 & $1,045,900$ \\
\hline Direct Condenser / Indirect Evaporator & 85,900 & $1,048,760$ \\
\hline Direct Condenser and Evaporator & 88,400 & $1,007,600$
\end{tabular}

Table 2 indicates that there is no permutation of a water cycle that will be economically competitive with an R-134a cycle over a 20-year horizon. The most attractive water cycle takes advantage of the cheaper, higher performance direct contact 
heat exchangers to accomplish both the evaporation and condensation functions; the benefit of this is significant but insufficient to overcome the very high cost of the centrifugal compressors. Over a longer expected life-cycle, the maximum allowable first cost will approach the actual first cost. However, the greater likelihood that contamination or fouling issues will degrade the performance of the water cycle may eliminate its advantage over the R-134a cycle. Note that Table 2 provides a convenient means of illustrating the results of this study. The greatest uncertainty in the economic analysis lies in predicting the first cost of a production chiller using water as a refrigerant. However, the maximum allowable first cost that can be tolerated depends only on the current first cost of a centrifugal chiller and the performance of the water and R-134a cycles; these are quantities that can be predicted with some degree of accuracy.

\section{OTHER ISSUES}

This project identified and explicitly modeled many of the significant challenges associated with an economically viable water as a refrigerant cycle, including the high volumetric flow/high pressure ratio compression process as well as the large, indirect contact heat exchangers that would be required. This last drawback may be alleviated somewhat by the possible use of direct-contact heat exchangers. However, there are many additional practical obstacles that are less easily quantified but still extremely important.

The use of direct-contact heat exchangers will come with some penalty associated with contamination within the refrigeration cycle. The possibility of the infiltration of air and other non-condensable gases, solids, and entrained liquids is increased. In particular, a direct-contact condenser used in conjunction with a cooling tower will allow air to be 
absorbed into the water as it circulates through the cooling tower. A significant amount of non-condensable gas in the refrigerant results in a loss of cycle performance and so the non-condensable gases must be purged. The condenser water associated with a 1000-ton chiller system would result in an $1800 \mathrm{cfm}$ volumetric flow rate of air that must be purged from the system, assuming that the water is completely saturated with air. Based on current vacuum pump technology, this flow rate would require nominally 100 horsepower which corresponds to nominally $10 \%$ of the input power and would therefore negate any COP benefit associated with the water cycle. In any case, the purging and filtration units required will add to the capital and operating costs of the system and therefore reinforce the conclusion illustrated by Table 2, that the water as a refrigerant cycle is not economically viable.

Another unique aspect of the water-based vapor compression refrigeration cycle is that the effect of any pressure loss in heat exchangers that operate between 0.01 and 0.1 atm are magnified greatly. Tube pitch in indirect contact heat exchangers and packing density in direct contact heat exchangers are critical design parameters and result in larger heat exchangers than might be expected based on the thermal loading alone.

Finally, there is some concern regarding the presence of droplets at the inlet to the compressor. Because the flow is accelerated to match the impeller blade velocity and angle, the static temperature drops. In a water vapor compressor this effect is quite significant and raises the possibility that condensation will occur at the nozzle exit. In order to avoid this possibility, it is necessary to add some superheat to prevent this situation. The penalty associated with superheat is very large for a water cycle. To first order, the penalty on cycle COP due to superheat is linear and decreases the COP by 
approximately 0.03 for each ${ }^{\circ} \mathrm{F}$ of superheat. However, there is some evidence from the power generation industry to indicate a supersaturated state may result in which case droplets will not form.

\section{SUMMARY}

This project investigated the economic viability of using water as the refrigerant in a 1000-ton chiller application. The most attractive water cycle configuration was found to be a flash-intercooled, two-stage cycle using centrifugal compressors and direct contact heat exchangers. Component level models were developed that could be used to predict the size and performance of the compressors and heat exchangers in this cycle as well as in a baseline, R-134a refrigeration cycle consistent with chillers in use today. A survey of several chiller manufacturers provided information that was used to validate and refine these component models.

The component models were integrated into cycle models that were subsequently used to investigate the life-cycle costs of both an R-134a and water refrigeration cycle. It was found that the first cost associated with the water as a refrigerant cycle greatly exceeded the savings in operating costs associated with its somewhat higher COP. Therefore, the water refrigeration cycle is not an economically attractive option to today's R-134a refrigeration system. There are a number of other issues, most notably the requirements associated with purging non-condensable gases that accumulate in a direct contact heat exchanger, which will further reduce the economic viability of the water cycle. 


\begin{abstract}
The purpose of this research was to investigate the economic feasibility of a water-based vapor compression chiller with a nominal capacity of 1000 tons. Low-level models of various potential cycle configurations have been constructed and the results of simulations for each cycle configuration are described. More detailed component level models were developed to accurately size equipment and predict system performance for the most attractive cycle configuration. These component models address issues that are particularly crucial in water as refrigerant cycles, such as compressor discharge superheat and refrigerant side pressure drop. Where possible, these component models were verified through comparison against the current state-of-the-art technology for large chillers using R-134a as a refrigerant. The capital cost and the expected operating costs are determined to quantify the payback time associated with using water as a refrigerant relative to traditional halocarbon refrigerants currently in use. Other issues that may have an economic impact on the feasibility of water as a viable alternative to R-134a such as the possibility of using direct contact heat exchangers, purging issues, and condensation at the compressor inlet have been discussed.
\end{abstract}




\section{Table of Contents}

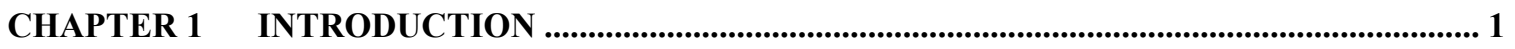

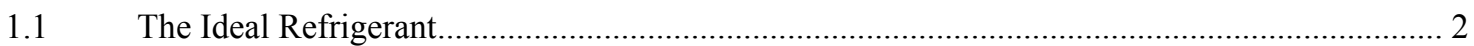

Water as a Refrigerant - Cycle Ramifications ............................................................ 4

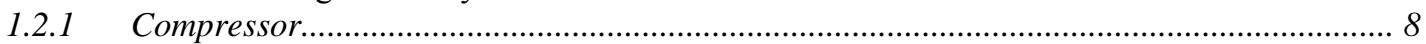

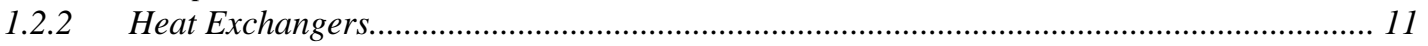

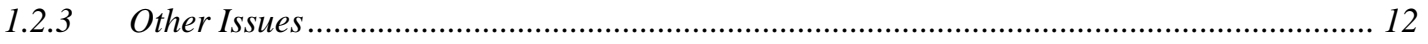

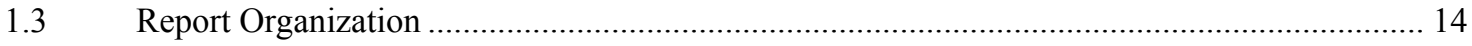

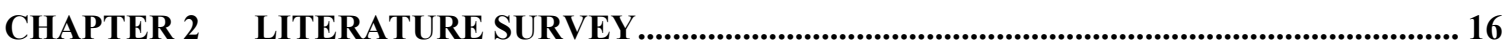

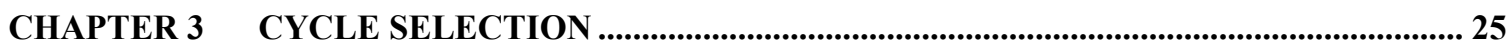

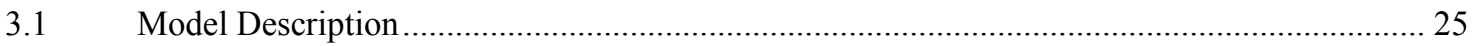

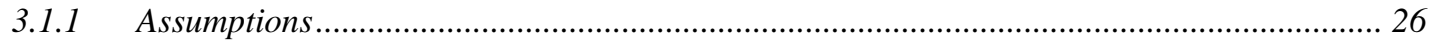

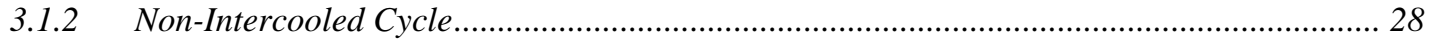

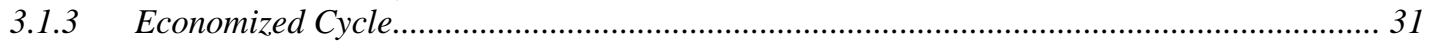

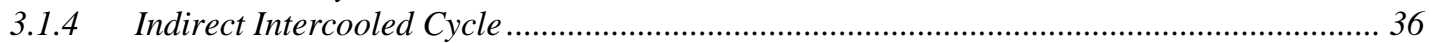

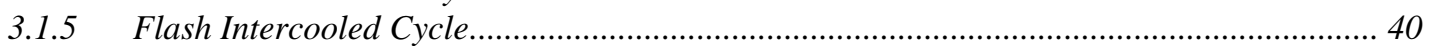

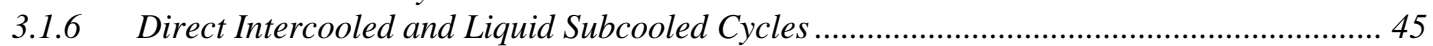

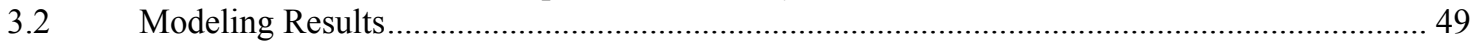

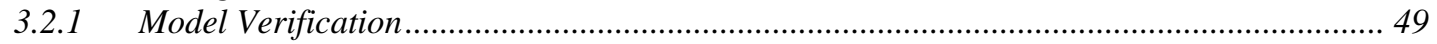

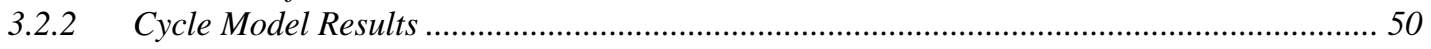

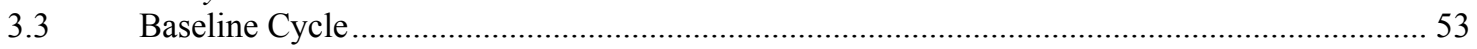

CHAPTER 4 SYSTEM MODEL ........................................................................................60

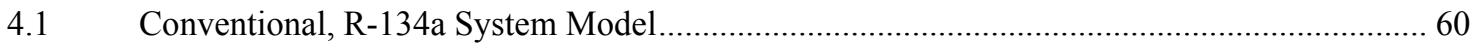

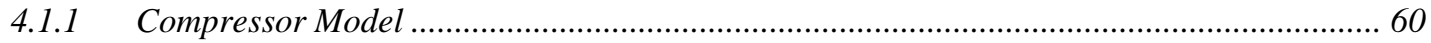

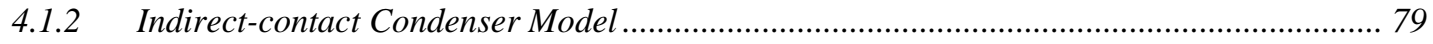

4.1.3 Indirect-contact Evaporator Model ........................................................................... 92

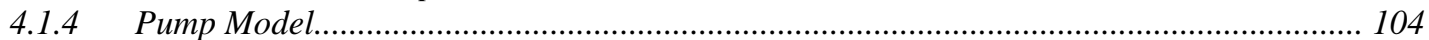

B.2 Baseline, Water System Models ................................................................................. 112

4.2.1 Compressor Model ................................................................................................ 112

4.2.2 Indirect-Contact Condenser Model ............................................................................. 121

4.2.3 Indirect-Contact Evaporator Model ...................................................................... 126

4.2.4 Direct-Contact Condenser Model.......................................................................... 130

4.2.5 Direct-Contact Evaporator Model............................................................................. 141

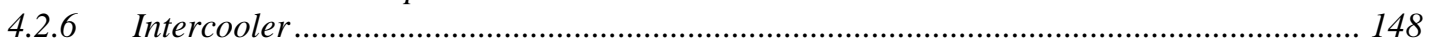

4.2.7 Pump Model............................................................................................................ 150

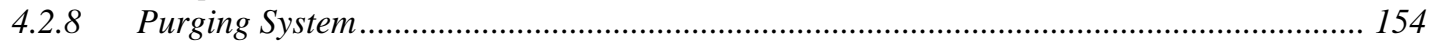

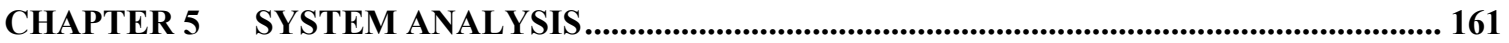

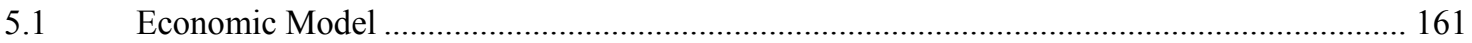

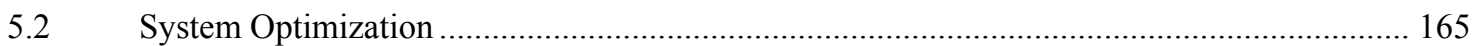

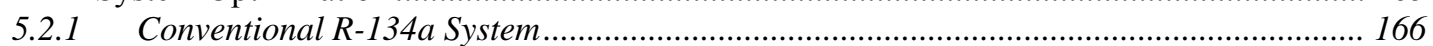

5.2.2 Water, Indirect-Contact Condenser/Indirect-Contact Evaporator System ........................ 169

5.2.3 Water, Direct-Contact Condenser/Indirect-Contact Evaporator System ........................... 173

5.2.4 Water, Indirect-Contact Condenser/Direct-Contact Evaporator System ........................... 175

5.2.5 Water, Direct-Contact Condenser/Direct-Contact Evaporator System............................. 177

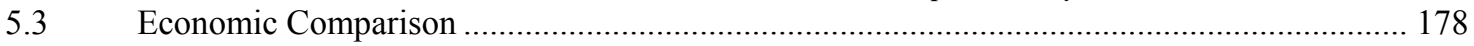

Specification of Preferred Cycle............................................................................ 182

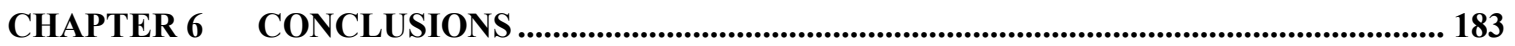

EES Code for four permutations of flash-intercooled water cycles: ............................................ 192

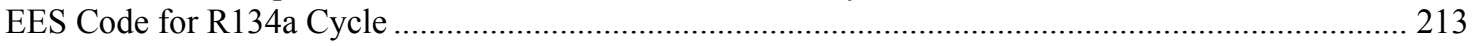




\section{List of Tables}

Table 1 Comparison of refrigerant characteristics (ASHRAE Handbook, 2002).............. 3

Table 2 Characteristics of water $v$. $R$-22 refrigeration cycle ....................................... 6

Table 3 ARTI specified water temperatures................................................................... 26

Table 4 Indexing convention for states within flash intercooled cycle ............................ 55

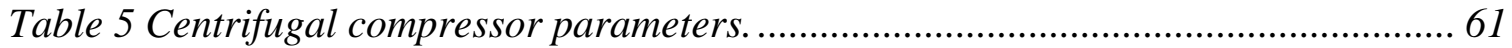

Table 6 Dimensions and performance of predicted $R-134$ a centrifugal compressor....... 77

Table 7 Comparison of compressors and models in real applications with UW - Madison

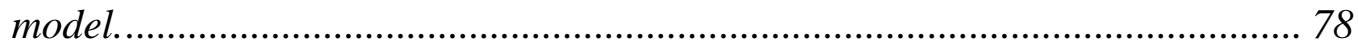

Table 8 Indirect-contact condenser operating parameters. .......................................... 80

Table 9 Indirect-contact condenser dimensions based on ARTI standard conditions...... 91

Table 10 Indirect-contact evaporator design parameters. ........................................... 92

Table 11 Indirect-contact evaporator dimensions based on ARTI standard conditions for $R-134 a$.

Table 12 Density and volumetric flow rates for the high and low pressure compressors using equal pressure ratio staging............................................................... 114

Table 13 Reynolds numbers for centrifugal compressor components with different refrigerants.

Table 14 Size and performance of centrifugal compressors for water and R-134a cycles.

Table 15 Cost of centrifugal compressors using water as a refrigerant.

Table 16 Comparison between discharge temperatures for water and R-134a as refrigerants.

Table 17 Tube heat transfer coefficient comparison between water and R-134a......... 124

Table 18 Indirect-contact condenser dimensions based on ARTI standard conditions for water.

Table 19 Water and R-134a condenser cost comparison. 126

Table 20 Evaporator tube heat transfer coefficient comparison. ................................... 128

Table 21 Comparison of water and $\mathrm{R}-134$ a evaporators. ........................................... 128

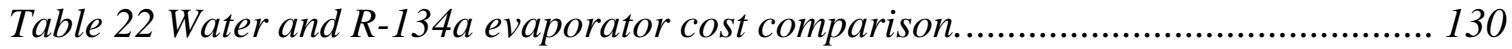

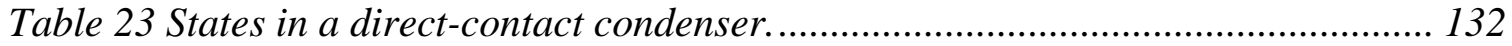

Table 24 Direct-contact condenser dimensions.......................................................... 140

Table 25 Price comparison between direct and indirect condensers. ............................ 141

Table 26 Indexing for states in direct-contact evaporator. .......................................... 142

Table 27 Evaporator dimensional comparison of water direct/indirect and R-134a cycles.

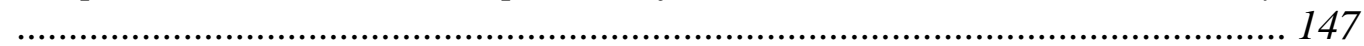

Table 28 Price comparison between direct and indirect evaporators............................ 148

Table 29 Comparison of sizes of direct-contact heat exchangers (packing material used).

Table 30 Comparison of pumping requirements for various heat exchanger configurations.

Table 31 Individual component costs. 166

Table 32 Comparison of average compressor size and cost with model predictions for

$R$-134a chiller.

Table 33 Comparison of industry average $R$-134a evaporator with model predictions. 168

Table 34 Comparison of industry average R-134a condenser with model predictions.. 169 
Table 35 Cost comparison of components in 1000-ton water (indirect condenser/indirect evaporator) and R-134a chiller cycles. ...................................................... 172

Table 36 Comparison of water (indirect condenser/indirect evaporator) and $R-134 a$ evaporators for 1000-ton chiller systems. ....................................................... 173

Table 37 Comparison of water (indirect condenser/indirect evaporator) and $R-134 a$ condensers for 1000-ton chiller systems...................................................... 173

Table 38 Cost comparison of components in 1000-ton water (direct condenser/indirect evaporator) and $R-134$ a chiller cycles. ....................................................... 174

Table 39 Comparison of water and R-134a indirect-contact evaporators for 1000-ton chiller systems........................................................................................ 174

Table 40 Comparison of direct-contact water condenser and R-134a indirect-contact condenser for 1000-ton chiller systems. ........................................................ 175

Table 41 Cost comparison of components in 1000-ton water and R-134a chiller cycles. ……................................................................................................... 176

Table 42 Comparison of direct-contact water evaporator and R-134a indirect-contact evaporator for 1000-ton chiller systems...................................................... 177

Table 43 Comparison of water and R-134a condensers for 1000-ton chiller systems... 177 Table 44 Cost comparison of components in 1000-ton water and R-134a chiller cycles.

Table 45 Comparison of direct-contact water evaporator and $R$-134a indirect-contact evaporator for 1000-ton chiller systems...................................................... 178

Table 46 Comparison of direct-contact water condenser and $R$-134a indirect-contact condenser for 1000-ton chiller systems. ........................................................ 178

Table 47 Results of 20-year life-cycle cost analysis.................................................... 179

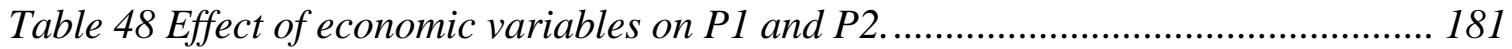

Table 49 Preferred cycle compressor specifications...................................................... 182 


\section{List of Figures}

Figure 1 Schematic of a conventional vapor compression refrigeration cycle.................. 4

Figure 2 Ideal water cycle and R-22 cycles shown on a pressure-volume diagram......... 5

Figure 3 T-s diagram illustrating difference between isentropic and polytropic efficiency.

Figure 4 Cycle schematic for two-stage cycle with no intercooling................................. 29

Figure 5 Temperature-entropy diagram of a two-stage cycle with no intercooling......... 29

Figure 6 Comparison of the cycle COP as a function of the number of compression stages using both polytropic and isentropic compression efficiencies for both water and $R-134$ a as a refrigerant in a non-intercooled cycle. ...................... 31

Figure 7 Schematic of a two-stage economized cycle. ................................................. 32

Figure 8 Schematic of a two-stage economized cycle. .................................................. 33

Figure 9 Expanded view of $1^{\text {st }}$ stage compressor discharge $/ 2^{\text {nd }}$ stage compressor suction states.

Figure 10 Cycle COP as a function of the number of compression stages for economized and non-intercooled cycles for water as a refrigerant.

Figure 11 Cycle COP as a function of the number of compression stages for economized and non-intercooled cycles for both water and $R-134 a$ as a refrigerant........ 36

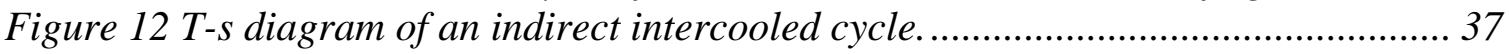

Figure 13 Schematic of a two-stage indirect intercooled cycle......................................... 38

Figure 14 Cycle COP as a function of the number of stages of compression for the indirect intercooled cycle (as well as the non-intercooled and economized cycles).

Figure 15 Cycle COP as a function of the number of compression stages for indirect intercooled, economized, and non-intercooled cycles for both water and $R$ 134 a as a refrigerant.

Figure 16 Schematic of a 2-stage flash intercooled cycle ("Process Water Bleed Off" configuration).

Figure 17 Schematic of a 2-stage flash intercooled cycle ("Condenser Water Bleed-off" configuration).

Figure 18 T-s diagram of a 2-stage flash intercooled cycle ("Process Water Bleed-off" configuration).

Figure 19 Expanded View of the 1st stage compressor discharge (2)/2nd stage compressor suction (3) states for the flash intercooled cycle.

Figure 20 Cycle COP as a function of the number of stages of compression for a flash intercooled cycle using the "Condenser Water Bleed Off" configuration compared with indirect intercooled, economized, and non-intercooled.

Figure 21 Cycle COP as a function of the number of compression stages for indirect intercooled, flash intercooled, economized, and non-intercooled cycles for both water and $R-134$ a as a refrigerant. 45

Figure 22 Schematic of a two-stage direct intercooled cycle......................................... 46

Figure 23 Schematic of a two-stage liquid subcooled cycle ............................................ 47

Figure 24 T-s diagram of a 2-stage direct intercooled cycle . ....................................... 47

Figure 25 COP as a function of the height of any standing refrigerant in the cycle for a 2stage direct intercooled cycle for water and $R-134 a$ as refrigerants. 48 
Figure $26 \mathrm{COP} / \mathrm{COP}$ Carnot based on the models developed here and the results described by van Orshoven et al. (1991) for an array of cycle configurations................ 50

Figure 27 T-s diagram of the baseline cycle selected for this project: A 2-stage flash intercooled cycle...... 54

Figure 28 COP vs. evaporator approach temperature difference for a water 2-stage flash intercooled cycle and a single stage $R$-134a cycle.......................................... 58

Figure 29 COP vs. condenser approach temperature difference for a water 2-stage flash intercooled cycle and a single stage $R$-134a cycle............................................58

Figure $30 \mathrm{COP} v$ s. condenser pressure drop for a water 2-stage flash intercooled cycle and a single stage $R-134$ a cycle...................................................................... 59

Figure 31 Schematic illustrating the components of a centrifugal compressor............... 62

Figure 32 Schematic illustrating the state points within a compressor alongside the corresponding $T$-s diagram ( $R$-134a cycle).

Figure 33 Velocity vector diagrams for the impeller blade hub and shroud inlets.......... 68

Figure 34 Vaned diffuser velocity profiles..................................................................... 75

Figure 35 Schematic of indirect-contact condenser model with $R-134 a$ as a refrigerant.80

Figure 36 Schematic of an indirect-contact evaporator.................................................. 93

Figure 37 Illustration of piping configuration for a 4-story 400,000 ft ${ }^{2}$ building......... 105

Figure 38 Pump curve illustrating the chilled water pump operating conditions for the

Model 15106 G (courtesy of Bell and Gossett, 2003). .................................... 110

Figure 39 Pump curve illustrating the condenser water pump operating for the Model 15108 G (courtesy of Bell and Gossett, 2003). 110

Figure 40 T-s diagram illustrating the savings of flash intercooling............................. 113

Figure 41 T-s diagram of water vapor centrifugal compressor.................................... 116

Figure 42 Mollier diagram illustrating the Wilson line (from El Wakil, 1984). ............. 119

Figure 43 COP vs. compressor suction superheat for a 2-stage flash intercooled cycle.

Figure 44 Plot of refrigerant pressure drop and COP variation with tube pitch........... 123

Figure 45 Plot of condenser shell diameter as a function of tube pitch......................... 124

Figure 46 Schematic of direct-contact condenser with fill media................................... 132

Figure 47 Direct-contact evaporator schematic.......................................................... 143

Figure 48 Operating conditions for condenser water pump for indirect-contact $\mathrm{HX}$ on the pump curve for Model 1510 8G (courtesy of Bell and Gossett, 2003)........... 152

Figure 49 Operating conditions for chilled water pump for indirect-contact $H X$ on the pump curve for Model 1510 6G (courtesy of Bell and Gossett, 2003)........... 153

Figure 50 Operating conditions for condenser water pump for direct-contact $\mathrm{HX}$ on the pump curve for Model 1510 8G (courtesy of Bell and Gossett, 2003)........... 153

Figure 51 Operating condition for chilled water pump for direct-contact HX on the pump curve for Model 1510 6G (courtesy of Bell and Gossett, 2003)..................... 154

Figure 52 Nitrogen solubility in water as a function of temperature and pressure (Bell and Gossett, 1998).................................................................................. 158

Figure 53 Approximate pump power requirement versus air flow rate. ........................ 159

Figure 54 Compressor size for an R-134a cycle as a function of refrigeration capacity reported by industry and predicted by model................................................ 167

Figure 55 Compressor cost for an R-134a cycle as a function of refrigeration capacity reported by industry and predicted by model.................................................. 168 
Figure 56 Operating cost as a function of evaporator pinch-point temperature difference for an indirect condenser/indirect evaporator water cycle.

Figure 57 First capital cost as a function of evaporator pinch-point temperature difference for an indirect condenser/indirect evaporator water cycle........... 170

Figure 58 Life-cycle cost as a function of evaporator pinch-point temperature difference for an indirect condenser/indirect evaporator water cycle.

Figure 59 Life-cycle cost as a function of condenser pinch-point temperature difference for an indirect condenser/indirect evaporator water cycle.

Figure 60 Life-cycle cost as a function of condenser pinch-point temperature difference for a direct condenser/indirect evaporator water cycle.

Figure 61 Life-cycle cost of a water cycle with an indirect-contact condenser and directcontact evaporator as a function of the evaporator pinch-point temperature difference.

Figure 62 Maximum allowable first cost as a function of the lifetime for a water cycle with a direct-contact condenser and direct-contact evaporator...................... 180

Figure 63 Screen capture of diagram window used for EES program............................ 213 


\section{NOMENCLATURE}

$\begin{array}{ll}\mathrm{A}_{\text {building }} & \text { Building Footprint } \\ \mathrm{A}_{\mathrm{HX}} & \text { Heat Transfer Surface Area } \\ \mathrm{AR} & \text { Aspect Ratio } \\ \mathrm{a}_{\mathrm{s}} & \text { Specific Surface Area } \\ \mathrm{c} & 1 \text { for Commercial; 0 for Residential Investment } \\ \mathrm{c}_{5} \mathrm{c}_{4} & \text { Diffuser Velocity Ratio } \\ \mathrm{C}_{\mathrm{F}} & \text { First Capital Cost } \\ \mathrm{C}_{\mathrm{F}, \text { water }} & \text { Water Cycle First Capital Cost } \\ \mathrm{C}_{\mathrm{F}, \mathrm{R}-134 \mathrm{a}} & \text { R-134a Cycle First Capital Cost } \\ \mathrm{Chilled}_{\text {Area }} & \text { Floor Area to be Refrigerated } \\ \dot{\mathrm{C}}_{\text {min }} & \text { Minimum Capacitance } \\ \dot{\mathrm{C}}_{\text {max }} & \text { Maximum Capacitance } \\ \mathrm{C}_{\mathrm{O}} & \text { Operating Cost } \\ \mathrm{C}_{\mathrm{O} \text {,water }} & \text { Water Cycle Operating Cost } \\ \mathrm{C}_{\mathrm{O}, \mathrm{R}-134 \mathrm{a}} & \text { R-134a Cycle Operating Cost } \\ \mathrm{CoolCapacity} & \text { Refrigeration Load } \\ \mathrm{COP} & \text { Coefficient of Performance } \\ \mathrm{COP} & \text { Carnot COP } \\ \mathrm{C}_{\mathrm{p}} & \text { Constant Pressure Specific Heat } \\ \mathrm{C}_{\mathrm{pr}} & \text { Diffuser Coefficient of Pressure Recovery } \\ \mathrm{C}_{\mathrm{r}} & \text { Capacitance Ratio } \\ \mathrm{c}_{\text {slip }} & \text { Refrigerant Slip Velocity } \\ \mathrm{CWB} & \text { Cooling Water Bleed-off Configuration } \\ \mathrm{d} & \text { Market Discount Rate } \\ \mathrm{D} & \text { Ratio of Down Payment to Initial Investment } \\ \mathrm{D}_{\mathrm{HX}} & \text { Shell Diameter } \\ \mathrm{d}_{\text {tip }} & \text { Impeller Blade Tip Diameter } \\ \mathrm{d}_{\text {tube,i }} & \text { Tube Inner Diameter } \\ \mathrm{d}_{\text {tube,o }} & \text { Tube Outer Diameter } \\ \mathrm{f} & \text { Friction Factor } \\ \mathrm{f} & \text { Fuel Inflation Rate } \\ \mathrm{g} & \text { Acceleration Due to Gravity } \\ \mathrm{h}_{1} & \text { Inlet Enthalpy } \\ \mathrm{h}_{2 \mathrm{~s}} & \text { Isentropic Exit Enthalpy } \\ \mathrm{H}_{\text {building }} & \text { Building Height } \\ \mathrm{h}_{\text {exit }} & \text { Exit Enthalpy } \\ \mathrm{h}_{\mathrm{fg}} & \text { Enthalpy of Vaporization } \\ \mathrm{htc} & \text { Heat Transfer Coefficient } \\ \mathrm{I} & \text { General Inflation Rate } \\ \mathrm{j} & \text { Indexed Variable } \\ \mathrm{k} & \text { Thermal Conductivity } \\ \mathrm{L}_{\text {building }} & \text { Building Length } \\ \mathrm{LCC} & \text { Life-Cycle Cost } \\ \mathrm{L}_{\mathrm{HX}} & \text { Shell Length } \\ & \end{array}$




\begin{tabular}{|c|c|}
\hline $\mathrm{L}_{\text {pipe }}$ & "Secondary" Refrigerant Piping Length \\
\hline $\mathrm{L}_{\text {pipe,eff }}$ & Effective Piping Length \\
\hline $\mathrm{L}_{\text {tube,total }}$ & Total Tube Length \\
\hline $\mathrm{m}$ & Annual Mortgage Interest Rate \\
\hline M & Ratio of Maintenance, Insurance, Other Incidental Costs to First Cost \\
\hline$\dot{\mathrm{m}}$ & Mass Flow Rate \\
\hline MACRS & Modified Accelerated Cost Recovery System \\
\hline $\mathrm{Ma}_{4}$ & Impeller Exit Mach Number $\left(\mathrm{Ma}_{\text {shroud }}\right)$ \\
\hline $\mathrm{Ma}_{\mathrm{r} \text { shroud }}$ & Refrigerant Mach Number Relative to Impeller at Shroud \\
\hline $\mathrm{Ma}_{\mathrm{r}, \mathrm{tip}}$ & Refrigerant Mach Number Relative to Impeller at Tip \\
\hline $\mathrm{Ma}_{\text {tip }}$ & Refrigerant Mach Number at the Impeller Tip \\
\hline $\mathrm{N}$ & Period of Economic Analysis (years) \\
\hline $\mathrm{N}_{1}$ & Number of Years Mortgage Payments Contribute to Analysis \\
\hline $\mathrm{N}_{2}$ & Number of Years Depreciation Contributes to Analysis \\
\hline $\mathrm{N}_{\text {baffles }}$ & Number of Baffles \\
\hline $\mathrm{N}_{\mathrm{D}}$ & Depreciation Lifetime (years) \\
\hline $\mathrm{N}_{\mathrm{L}}$ & Term of Loan (years) \\
\hline $\mathrm{N}_{\text {pass }}$ & Number of Tube Passes \\
\hline $\mathrm{n}_{\mathrm{s}}$ & Compressor Specific Speed \\
\hline NSC & Number of Stages of Compression \\
\hline NTU & Number of Transfer Units \\
\hline $\mathrm{N}_{\text {tubes }}$ & Number of Tubes \\
\hline $\mathrm{N}_{\text {tubes,vert }}$ & Number of Tubes Stacked Vertically in Cross-section \\
\hline $\mathrm{N}_{\text {tubes,Xsection }}$ & Number of Tubes in Shell Cross-section \\
\hline $\mathrm{Nu}$ & Nusselt Number \\
\hline ODP & Ozone Depletion Potential \\
\hline $\mathrm{GWP}_{100 \mathrm{yr}}$ & Global Warming Potential (100-yr) \\
\hline $\mathrm{p}$ & Property Tax Rate \\
\hline $\mathrm{P}_{1}$ & First Cost Present Worth Factor \\
\hline $\mathrm{P}_{2}$ & Operating Cost Present Worth Factor \\
\hline $\mathrm{p}_{\text {condenser }}$ & Condenser Saturation Pressure \\
\hline $\mathrm{p}_{\text {chw }}$ & Average Chilled Water Pressure \\
\hline $\mathrm{p}_{\mathrm{cw}}$ & Average Condenser Water Pressure \\
\hline pevaporator & Evaporator Saturation Pressure \\
\hline Pitch \TubeOD & Tube Pitch-to-Tube Outer Diameter Ratio \\
\hline $\operatorname{Pr}$ & Prandtl Number \\
\hline PWB & Process Water Bleed-off Configuration \\
\hline PWF() & Present Worth Factor \\
\hline$\dot{\mathrm{q}}_{\text {evap }}$ & Evaporator Load \\
\hline $\mathrm{r}$ & Effective State and Federal Income Tax Rate \\
\hline$r_{c}$ & Compression Ratio \\
\hline $\mathrm{Re}$ & Reynolds Number \\
\hline $\mathrm{r}_{\text {hub }}$ & Hub Radius \\
\hline$r_{\text {hub-to-tip }}$ & Hub-to-Tip Radius Ratio \\
\hline $\mathrm{r}_{\text {shroud }}$ & Shroud Radius \\
\hline$r_{\text {tip }}$ & Impeller Tip Radius \\
\hline
\end{tabular}




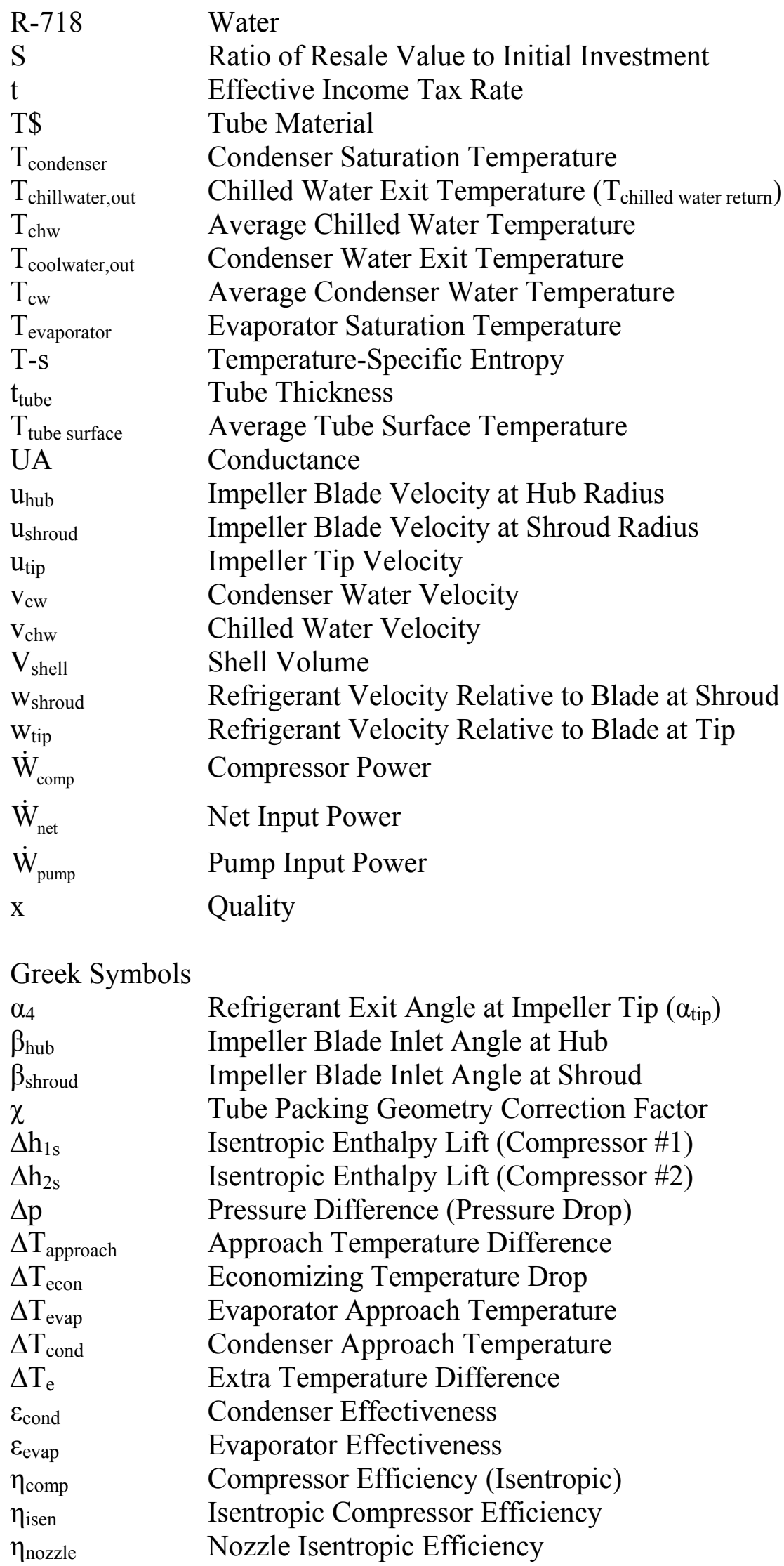




$\begin{array}{ll}\eta_{\text {pack }} & \text { Packing Material Efficiency } \\ \eta_{\text {poly }} & \text { Polytropic Compressor Efficiency } \\ \eta_{\text {pump }} & \text { Isentropic Pump Efficiency } \\ \eta_{\mathrm{TT}, \mathrm{imp}} & \text { Total-to-Total Impeller Efficiency } \\ \eta_{\mathrm{vs}} & \text { Vaneless Space Isentropic Efficiency } \\ \Gamma & \text { Film Reynolds Number } \\ \mu & \text { Dynamic Viscosity } \\ v & \text { Kinematic Viscosity } \\ \rho & \text { Density } \\ \sigma & \text { Impeller Blade Slip Factor }\end{array}$

$\begin{array}{ll}\text { Subscipts } & \\ \text { o } & \text { Stagnation Property } \\ \theta & \text { In the Tangential Direction } \\ \text { atm } & \text { Atmospheric } \\ \mathrm{cw} & \text { Condenser Water } \\ \mathrm{chw} & \text { Chilled Water } \\ \mathrm{f} & \text { Film } \\ \text { is } & \text { Isentropic } \\ \mathrm{l} & \text { Saturated Liquid } \\ \mathrm{ref} & \text { Refrigerant side } \\ \text { req } & \text { Required } \\ \mathrm{s} & \text { Isentropic } \\ \text { tube bank } & \text { Pertaining to the Tube Bank } \\ \mathrm{v} & \text { Saturated Vapor }\end{array}$




\section{CHAPTER 1 INTRODUCTION}

Water is an abundant constituent of the earth's biosphere and it penetrates every aspect of our industrial society. Water finds extensive use as a process fluid (for example in distillation, freeze concentration, or drying processes), as a heat transfer or energy storage medium (for example in central or district heating and cooling systems, stratified chilled water tanks, and ice storage systems), and as a working fluid in power cycles (most commonly in the Rankine power generation cycle).

In refrigeration applications, the use of water as a refrigerant has been limited to absorption systems built around a binary fluid comprised of lithium bromide and water. Absorption refrigeration represents a relatively small percentage of the total airconditioning market in the U.S. The HVAC market in the U.S. is dominated by vapor compression-based refrigeration systems using synthetic refrigerants. A number of factors limit the attractiveness of water-based vapor compression refrigeration cycles. One key factor that makes the use of water as a refrigerant challenging is the process of compressing low pressure, high specific volume vapor to a pressure (and therefore temperature) that is high enough to allow heat rejection from the system.

The use of water as a refrigerant in vapor compression systems does offer several potentially significant advantages. First, water is an ecologically sound refrigerant with no Ozone Depletion Potential (ODP) and a Global Warming Potential $\left(\mathrm{GWP}_{100 \mathrm{yr}}\right)$ less than 1, compared to GWP $100 y$ of 1300 for R-134a (Devotta, 2000). Water itself is low cost and readily available. Also, a water-based refrigeration system could be used in conjunction with direct-contact condensers and evaporators; thereby, serving the role of both refrigerant and primary heat transfer fluid. The elimination of conventional indirect- 
contact heat exchangers can reduce system capital cost, environmental impacts, and increase cycle performance. These advantages warrant a thorough investigation of waterbased refrigeration cycles in order to identify any non-traditional vapor compression cycle configurations that are particularly economically viable and also to determine whether the above-mentioned advantages associated with using direct-contact heat exchangers is sufficient to overcome the disadvantages relevant in the compression process.

\subsection{The Ideal Refrigerant}

The ideal refrigerant will produce the maximum cooling capacity for the minimum input power. This is not the only requirement of a refrigerant in today's world. A good refrigerant should also be safe for the environment and non-toxic (particularly in air-conditioning applications). The desirable characteristics of a refrigerant include, but are not limited to the following:

- high latent heat of vaporization

- reasonable working pressures

- non-toxic

- stable

- non-flammable

- no environmental impacts (no ozone depletion or global warming potential)

- low cost 
- good material compatibility

- readily available

- $\quad$ high theoretical coefficient of performance (COP)

- $\quad$ high thermal conductivity

- low viscosity

- low heat of compression

Table 1 compares several key characteristics for a few common synthetic refrigerants and some natural refrigerants, including water.

Table 1 Comparison of refrigerant characteristics (ASHRAE Handbook, 2002).

\begin{tabular}{|l|c|c|c|c|}
\hline & COP $_{\text {theoretical }}$ & GWP (100 yr) & ODP & $\begin{array}{c}\text { ASHRAE } \\
\text { Group } \\
\text { (Std34) }^{2}\end{array}$ \\
\hline $\mathrm{R}-11$ & 9.10 & 4600 & 1.0 & $\mathrm{~A} 1$ \\
\hline $\mathrm{R}-22$ & 8.48 & 1700 & 0.034 & $\mathrm{~A} 1$ \\
\hline $\mathrm{R}-134 \mathrm{a}$ & 8.47 & 1300 & 0 & $\mathrm{~A} 1$ \\
\hline $\mathrm{R} 123$ & 8.91 & 120 & 0.012 & $\mathrm{~A} 1$ \\
\hline Water & 8.39 & $<1$ & 0 & $\mathrm{~A} 1$ \\
\hline Ammonia & 8.78 & 0 & 0 & $\mathrm{~B} 2$ \\
\hline $\mathrm{CO}_{2}$ & N/A & 1 & 0 & $\mathrm{~A} 1$ \\
\hline
\end{tabular}

1. Theoretical COP compared between the ARTI specified condenser and evaporator temperatures of $95^{\circ} \mathrm{F}$ and $44^{\circ} \mathrm{F}$, respectively. The critical temperature of $\mathrm{CO}_{2}$ is below the condenser temperature.

2. Letter "A" or "B" refers to toxicity class (A - TLV $>400 \mathrm{ppm}$; B - TLV $<400 \mathrm{ppm}$ ); number 1-3 refers to flammability class $\left(1-\right.$ shows no flame propagation; $2-\mathrm{LFL}>0.10 \mathrm{~kg} / \mathrm{m}^{3}, \mathrm{~h}_{\mathrm{c}}<19,000$ $\mathrm{kJ} / \mathrm{kg} ; 3-\mathrm{LFL}>0.10 \mathrm{~kg} / \mathrm{m}^{3}, \mathrm{~h}_{\mathrm{c}}>19,000 \mathrm{~kJ} / \mathrm{kg}$ ) (ASHRAE Std 34)

Notice that the theoretical COP of water is competitive with synthetic refrigerants, it is environmentally attractive, and has the practical characteristics of being nonflammable and non-toxic. 


\subsection{Water as a Refrigerant - Cycle Ramifications}

Water fulfills all of the fundamental requirements of a refrigerant (McLinden, 1987); it is chemically stable, non-toxic, non-flammable, and environmentally benign. However, water has thermophysical characteristics that differ substantially from other refrigerants and result in a refrigeration cycle with some unique characteristics. Figure 1 illustrates a conventional vapor compression cycle operating between a nominal saturated evaporator temperature of $44^{\circ} \mathrm{F}$ and a saturated condensing temperature of $95^{\circ} \mathrm{F}$. Figure 2 illustrates the ideal cycles executed by water and a more conventional refrigerant, R-22, on a pressure-specific volume (log p-log v) diagram.

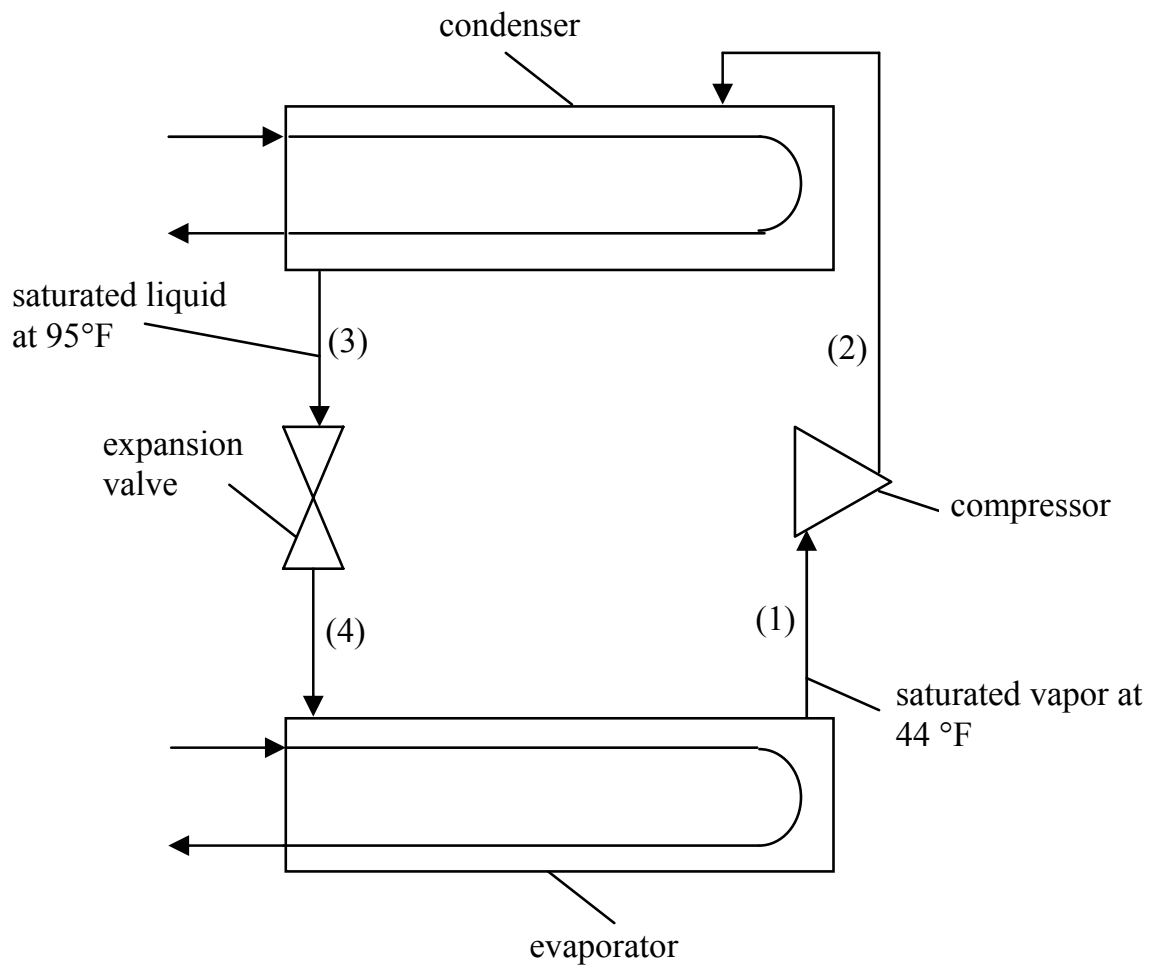

Figure 1 Schematic of a conventional vapor compression refrigeration cycle. 


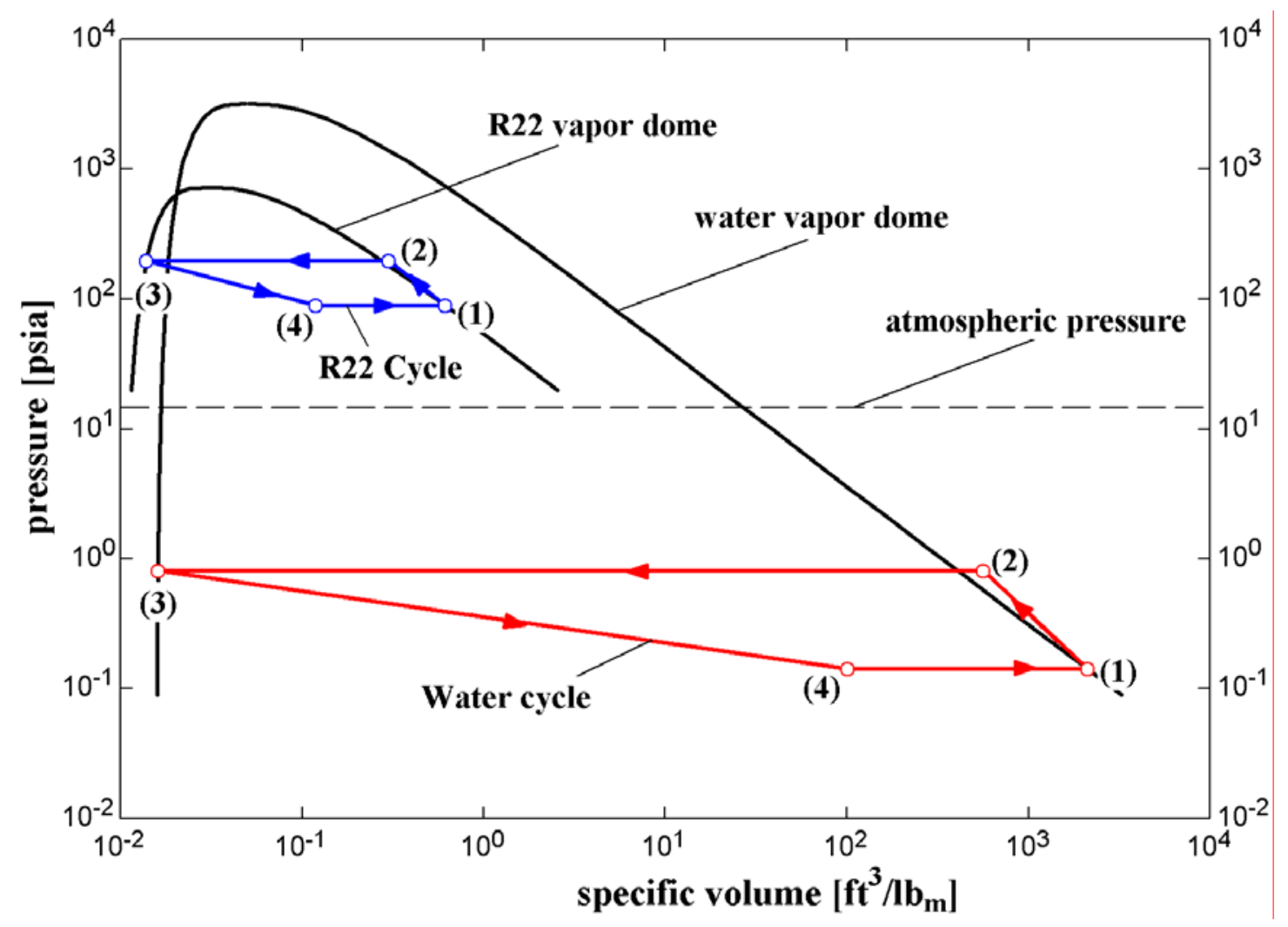

Figure 2 Ideal water cycle and R-22 cycles shown on a pressure-volume diagram.

Notice that the water refrigeration cycle operates at significantly lower pressures and with a considerably higher specific volume at the compressor suction. The required pressure ratio is also larger for the water-based cycle although the absolute pressure difference represented by this pressure ratio is smaller. These characteristics are summarized in Table 2 in the context of a 1000-ton capacity cycle. 
Table 2 Characteristics of water v. R-22 refrigeration cycle.

\begin{tabular}{|c|c|c|}
\hline Quantity & Water & R-22 \\
\hline Theoretical coefficient of performance, COP & 8.39 & 8.48 \\
\hline Carnot Coefficient of Performance, $\mathrm{COP}_{\text {Carnot }}$ & 9.88 & 9.88 \\
\hline $\mathrm{COP} / \mathrm{COP}_{\text {Carnot }}$ & 0.85 & 0.86 \\
\hline Compressor isentropic discharge temperature, $\mathrm{T}_{2 \mathrm{~s}},{ }^{\circ} \mathrm{F}$ & 313 & 118 \\
\hline Throttling loss, $\left(\mathrm{s}_{4}-\mathrm{s}_{3}\right) / \mathrm{Q}, \mathrm{R}^{-1}$ & $4.7 \mathrm{e}-6$ & $2.8 \mathrm{e}-5$ \\
\hline Superheat loss, $\mathrm{S}_{\text {gen,sh }} / \mathrm{Q}^{1}, \mathrm{R}^{-1}$ & $2.83 \mathrm{e}-4$ & $3.5 \mathrm{e}-5$ \\
\hline Compressor inlet specific volume, $\mathrm{v}_{1}, \mathrm{ft}^{3} / \mathrm{lbm}$ & 2111 & 0.61 \\
\hline Compressor suction pressure, $\mathrm{P}_{1}$, psia & 0.14 & 89 \\
\hline Compressor saturated suction temperature, $T_{1, \text { sat }},{ }^{\circ} \mathrm{F}$ & 44 & 44 \\
\hline Compressor discharge pressure, $\mathrm{P}_{2}$, psia & 0.82 & 196 \\
\hline Compressor saturated discharge temperature, $\mathrm{T}_{2, \text { sat }},{ }^{\circ} \mathrm{F}$ & 95 & 95 \\
\hline Compression ratio, PR & 5.75 & 2.2 \\
\hline Pressure lift, $\mathrm{P}_{2}-\mathrm{P}_{1}$, psia & 0.7 & 107 \\
\hline Compressor inlet volumetric flow rate, $\mathrm{ft}^{3} / \mathrm{s}$ & 6900 & 29 \\
\hline Isentropic enthalpy difference across compressor, $\Delta \mathrm{h}_{\mathrm{s}}$, Btu/lbm & 121 & 8.3 \\
\hline
\end{tabular}

1. $\mathrm{S}_{\mathrm{gen}, \mathrm{sh}}$ is the entropy generated by the irreversible de-superheat process

Table 2 is valid only for the simple, ideal vapor compression cycle illustrated in

Figures 1 and 2; yet it illustrates some of the challenges that surround the use of water as a refrigerant. The important conclusions that can be drawn from Table 2 are listed below.

o The ideal COP of water is comparable to that of R-22. This COP increases as the evaporator temperature increases; the intrinsic thermodynamic properties of water make it a poor refrigerant when operating close to its triple point, but these disadvantages do not persist at medium and higher temperatures.

o The specific volume of the fluid entering the compressor is much higher for the water cycle (3500x). Therefore, the volumetric flow rate through the compressor is also much higher $(240 \mathrm{x})$ even though the mass flow rate required is smaller.

o The pressure ratio required by the water cycle is much higher than that for the R-22 cycle (2.6x). 
o The pressure lift across the water compressor is much lower than for the R-22 compressor $(150 \mathrm{x})$. The small pressure difference for water implies reduced forces and suggests that it may be possible to use light construction materials because both axial and radial loads on the bearings related to the aerodynamic forces will be relatively small.

o The theoretical compressor discharge temperature is much higher for the water cycle and therefore the superheat loss is larger than for the R-22 cycle (8x). As a result, effort aimed at reducing the superheat loss through advanced cycle configurations is anticipated to have a large impact on cycle efficiency.

o The entropy generated by the throttling process as shown for water is much lower than that generated by the desuperheat process $(1 / 60 x)$. As a result, any effort aimed at replacing the throttle with a more isentropic device, such as a work-extracting expander, will result in only minimal performance gain.

o The sub-ambient pressures that characterize the water cycle create the potential for air infiltration. In a practical cycle, some provision must be made to constantly remove noncondensible gases from the refrigerant and prevent it from building up in the cycle. This will require additional hardware and result in a parasitic power loss.

o In many commercial air-conditioning applications, water is used as the heat transfer medium in both the condenser and evaporator. Therefore, waterbased refrigeration systems can be used in conjunction with direct-contact evaporators and condensers. This can reduce the complexity and size of the 
heat exchangers leading to a reduction in capital cost with equal to or better performance.

\subsubsection{Compressor}

The largest obstacle facing the development of water-based refrigeration cycles is associated with the compressor. Compressors that simultaneously satisfy the requirements of high pressure ratio, high volumetric flow capacity, high efficiency, and low capital cost are not currently available. In order to meet the stated project objective of 'assessing feasibility', it is important that the models developed here for simulating the compression hardware be physically realistic and account for issues related to the mechanical aspects of the compressor; in particular its very large size.

The flow capacity of positive displacement compressors is small relative to the needs of vacuum water vapor compression. The cyclic motion of reciprocating compressors results in large accelerations and decelerations that limit speed and therefore the volumetric throughput. The relative motion between the piston and cylinder results in unavoidable frictional losses that become large in relation to the power required to compress the relatively small amount of low density fluid that can be processed during each stroke. Other types of positive displacement compressors such as screw, rolling piston, liquid ring, rotating vane, trochoidal and scroll devices also lack the flow capacity and low-pressure efficiency that is required by a water vapor refrigeration cycle. Consequently, positive displacement compressors will not be considered or modeled during this project.

A more promising alternative for water vapor compression is the dynamic compressor. Axial and centrifugal compressors can be used to efficiently achieve both 
large pressure ratio and large volumetric flow rates (Cohen, et al, 1987). In a dynamic compressor, the work transmitted to the fluid per unit mass (the head rise) is related to the change in the angular momentum of the flow as it passes through the impeller. The blade velocities that can be achieved therefore limit the head rise that can be imparted. Centrifugal forces, compressibility effects, and rotordynamic limitations all conspire to restrict the enthalpy rise per compression stage to nominally 20-30 Btu/l $\mathrm{b}_{\mathrm{m}}$ (Neerken, 1980), which is significantly less than the required value listed in Table 2. This implies that multi-staging must be used and, given the large superheat loss described earlier, it seems likely that intercooling will also be required.

There are several potential advantages associated with the use of water in a vapor compression refrigeration system. Unfortunately, efforts to implement such a system have been impeded by the lack of a suitable water vapor compressor. Therefore, the Air Conditioning and Refrigeration Technology Institute (ARTI) sponsored a Phase I project during which Concepts ETI, Inc. carried out a scoping study in order to determine compressor configurations best suited to water-based vapor compression refrigeration systems (Wight et al, 2000). The results of this study showed that very large compressor impellers with only moderate efficiencies could be achieved.

In particular, the scoping calculations indicated that single stage centrifugal compressors were unattractive due to low efficiency (less than $75 \%$ for the radial bladed unit), very large impellers (20 ft in diameter), and very high tip velocities $(2200 \mathrm{ft} / \mathrm{sec})$. The high tip velocities imply large stresses and high Mach numbers and therefore will require considerable technical development time and capital cost. A two stage centrifugal compressor was also evaluated and the results were slightly better. This 
configuration could achieve efficiencies of nominally $80 \%$, required large impeller diameters, but had somewhat reduced tip velocities (nominally $1600 \mathrm{ft} / \mathrm{sec}$ ) suggesting a more technically achievable compressor.

The more promising compressor technology identified by the scoping study, in terms of total geometric size and performance, was a multistage axial compressor configuration consisting of between $6 \& 7$ stages without intercooling. The efficiency of this type of compressor was nominally $82 \%$ and the impeller diameter and tip speeds were much more reasonable, $4.5 \mathrm{ft}$ and $1400 \mathrm{ft} / \mathrm{sec}$, respectively. Although the axial flow compressor was characterized by high compressor efficiency, the lack of intercooling resulted in very high superheat losses; therefore, the overall system efficiency was low. Also, axial compressors are extremely sophisticated and expensive devices to manufacture when compared to centrifugal compressors. The use of axial compressors is generally limited to high unit cost devices such as jet engines and power plants. In contrast, centrifugal compressors are sufficiently inexpensive to allow their use in very large capacity refrigeration systems today. The stated objective of this project is to develop a credible economic analysis of alternative water-based refrigeration cycles. Given the inherently high cost of axial compression systems, it seems likely that a multistage compression process using centrifugal compressors will be more economically attractive and therefore this compression technology provides the focus of the compressor modeling carried out in this project.

The results of the Concepts ETI study were not sufficiently optimistic to justify investing large amounts of resources in pursuing the development of a water-based system utilizing a conventional vapor compression cycle configuration. However, a 
further study is warranted in order to determine whether more unique cycles might be economically viable. This report presents the results of this additional study. In particular, cycles that directly address the large superheat loss within a water refrigeration cycle via various types of intercooling configurations are investigated. Also, those cycles that fully exploit the potential for direct-contact evaporation and condensation are thoroughly examined and an economic analysis is prepared in order to determine whether this unique advantage for the water as a refrigerant cycle is sufficient to balance the cost of the large centrifugal compressors that are required.

\subsubsection{Heat Exchangers}

The use of water as a refrigerant allows the possibility of choosing either direct or indirect-contact heat exchangers. Direct-contact heat exchange is an option because water is an effective and convenient heat transport fluid and because it is non-toxic. Regardless of the type of heat exchanger that is selected, the extremely low pressures at which a water cycle operates and the large volumetric flow rates make pressure drop an important consideration that must be considered when assessing the feasibility of using water as a refrigerant.

On the basis of economics, the size of a heat exchanger for a high pressure refrigerant like R-134a is largely determined by the approach temperature. Generally, decreasing approach temperatures leads to increased heat exchanger cost. However, it is important to realize that what is a reasonable and even negligible pressure drop for an R134a heat exchanger would be devastating to the $\mathrm{COP}$ of a water cycle. For water, a very low pressure refrigerant, pressure drop is a factor that dominates the size of the heat exchanger and dictates its aspect ratio. The primary means of minimizing pressure drop 
is through the reduction of the refrigerant side velocity, which translates into a larger heat exchanger. This is a major difference between water as a refrigerant and more typical high pressure refrigerants and the heat exchanger design process for a water system is significantly different as a consequence. Models capable of carefully examining this effect for both direct and indirect-contact heat exchangers in a water cycle are described in this report.

\subsubsection{Other Issues}

On the surface, the ability to use direct-contact heat exchangers appears to translate into a substantial capital cost reduction when compared to indirect-contact heat exchangers. Upon closer inspection, however, the use of a direct-contact condenser introduces a potentially significant problem in accumulating noncondensables in the system. These noncondensables must be continuously purged in order to maintain a high cycle COP as they represent an additional compressor load and also degrade the heat exchanger performance. Water that is in intimate contact with air as it passes through a cooling tower rejecting heat will absorb significant quantities of air. This air will pass out of solution after it is injected into the condenser and accumulate in the refrigeration cycle. A significant amount of power will be required to remove the noncondensables from the system at the very low pressures associated with the refrigeration cycle and return them to atmospheric pressure. Issues related to noncondensable gas purging are explored in more detail in Section 4.2.8, although the cost of purging is not factored into the overall economic analysis because of the uncertainty in estimating the rate at which noncondensables enter the system. Also, it is understood that if water is found to 
compare poorly with other refrigerants without considering the cost of purging then this comparison will only get worse when purging is considered.

Refrigerant filtration is an issue that will also become important in open cycles, i.e. cycles that have direct-contact heat exchangers. With cooling towers will come foreign contaminants, such as solids in suspension and other contaminants.

Other issues that are somewhat unique to water as a refrigerant include blade erosion due to saturated liquid-vapor mixtures at the compressor impeller inlet and the cycle's sensitivity to pressure loss in piping and hydrostatic pressure variations.

Blade erosion may be an important consideration for water as a refrigerant. As the saturated vapor passes through the compressor inlet nozzle it accelerates and therefore its static temperature and pressure drops. Therefore, it is possible that the inlet to the compressor impeller may lie within the vapor dome; this introduces the possibility of condensation and therefore of small liquid droplets forming and eroding the impeller blades. It will be shown in this report that the propensity for this to occur is higher in a water as a refrigerant cycle and the amount and cycle impact of the superheat required to prevent this is also higher. With impeller blade diameters measured in feet rather than inches, maintenance costs associated with blade erosion will be much higher than with other refrigerants. Anecdotal evidence related by chiller manufacturers suggests that it may be possible for high quality saturated liquid-vapor mixtures to exist at the inlet to centrifugal compressor impeller without causing significant damage. Also, experience from the power generation industry provides some insight into how far into the vapor dome steam can penetrate as it leaves a power turbine prior to causing damage related to condensation. The so-called "Wilson line" (an imaginary line that runs within the vapor 
dome parallel to the saturated vapor line) has been established as a way of predicting whether condensation will occur within a turbine rotor and cause erosion damage. Condensation at the compressor inlets is explored in greater detail in Section 4.2.1.3.

A final issue that is unique, at least in terms of its magnitude, to water vapor refrigeration cycles is pressure loss due to the flow of high specific volume vapor through any practically sized piping or flow passages of any sort. Care must be taken to minimize length and maximize cross-sectional area in any region within the system where there is vapor flow. Pressure loss in any portion of a water cycle has a much greater impact than it would on a high pressure refrigerant cycle. This issue is explored more thoroughly in the sections describing the heat exchanger design and modeling.

\subsection{Report Organization}

This report consists of six chapters and two appendices. The background literature that was acquired and examined as part of carrying out this work is listed in Chapter 2. Chapter 3 presents a comprehensive evaluation of several possible cycle configurations using a common set of assumptions. The result of this evaluation is the selection of the most attractive, baseline cycle configuration which is subsequently modeled in more detail in Chapters 4 and 5. Chapter 4 presents a detailed description of each of the component level models that are developed in order to provide a more indepth analysis of the baseline cycle. The component level models provide estimates of the physical size, performance, and capital cost of the most important components that are required to implement the baseline cycle as well as a conventional R-134a vapor compression cycle that serves as a benchmark. Chapter 5 exercises the system model that results from integrating the various component models with the thermodynamic cycle 
model. The baseline cycle is optimized by selecting the size of the components that minimize the overall cost associated with purchasing and operating a chiller over its life. Four configurations of the baseline water refrigeration cycle are considered, corresponding to the four possible permutations of direct and indirect-contact heat exchangers. The benchmark R-134a cycle is also optimized in this way in order to provide a reasonable basis of comparison. Finally, the break-even capital cost of the most attractive water vapor cycle is determined; this is the premium that the operator can afford to pay for a water cycle as compared with the R-134a machine due to its somewhat higher performance. This analysis is independent of the assumptions for the capital cost of the water cycle (which requires some significant extrapolations from the current stateof-the-art) and therefore provides a more concrete indication of the economic viability of the water cycle. The conclusions of the report are summarized in Chapter 6. 


\section{CHAPTER 2 LITERATURE SURVEY}

This chapter is a review of the literature describing previous work that has considered the use of water as a refrigerant in a vapor compression cycle. This survey was limited to the subjects of refrigeration cycle analysis, refrigeration cycle comparison, alternative refrigerants, vapor compression refrigeration cycle components, and economics. A complete list of the literature reviewed during the course of this project is included in References. A description of those papers that were found to be most useful for this project follows.

Abdel-Khalik, S., Ghiaasiaan, S., Ren, W., Schoonover, K., 1996, “Mechanistic modeling of desuperheater performance", ISA Transactions, pp. 45-51.

This paper describes the development of a 3-D model of a direct-contact heat exchanger. The results of the model show that the heat transfer coefficient is largely dependent on droplet size. It showed that the smaller droplets tended to evaporate more quickly, but that cooling rate dropped quickly over time as the droplets evaporated.

Banquet, F., Degueurce, B., Denisart, J.P., Favrat, D., 1984, "Use of twin screw compressor for steam compression", $2^{\text {nd }}$ Int'l Symposium on the Large Scale Applications of Heat Pumps, pp. 189 - 196.

This paper explores the idea of using screw compressors in vapor compression cycles involving steam compression. The volumetric flow rates for the compressors 
described in this paper are $\sim 250$ times lower than those necessary for the 1,000 ton refrigeration application considered in this project.

Beckman, W.A., Klein, S.A., Van Orshoven, D, 1993, "The use of water as a refrigerant - an exploratory investigation", Journal of Energy Resources Technology, ASME Transactions, v. 115 , pp. $257-263$.

This paper provides a comprehensive look at the thermodynamic cycle implications associated with using water as a potential refrigerant. The COP values for a water-based cycle are compared with those of more conventional refrigerants for several cycle configurations. The problem of compression is described. The paper includes a qualitative examination of the relative merit of the various competing technologies.

Blaise, J.C., Yuan, Q.S. , 1988, "Water - a working fluid for CFC replacement”, Rev. Int. Froid, v. 11, pp. $243-247$.

This paper explores the use of water as the working fluid in heat pumps utilizing vapor compression cycles. The cycle considered uses direct water injection in the compressor to minimize the superheat generated during the compression process. The heat exchangers are both assumed to be indirect-contact with a shell-and-tube geometry. The expansion valve is coupled to a liquid level detector to maintain a certain level in the heat exchanger reservoirs. Yuan and Blaise concluded that water fits well as a working fluid in heat pumps, although there is room for improvement in the compression process as well as in the intercooling process. 
Boehm, R., Brickman, R., 1995, "Numerical simulation and comments on sieve tray spray-column direct-contact heat exchangers", ASME Journal of Heat Transfer, v. 317, n. 1, pp. 459-467.

This paper gives insight into the heat transfer characteristics of two immiscible liquids in direct-contact with each other. Although, most of the information in this paper is not relevant to this project, it does suggest that packing material significantly improves heat transfer in direct-contact heat exchangers.

Braun, J.E., 1988, "Methodologies for the design and control of central cooling plants", Ph.D. Thesis, University of Wisconsin - Madison.

This thesis provides information on centrifugal compressor size and performance in a chiller application utilizing halocarbon refrigerants. The study includes field data collected at the Dallas/Ft. Worth Airport central plant chilling systems that was used to verify the component models developed in this project. The specific information is valuable and extensive, including dimensions and estimates of heat transfer coefficients for indirect-contact condensers and evaporators in a conventional refrigeration cycle. 
DeAndres, M., Hoo, E., Zangrando, F., 1996, "Performance of direct-contact heat and mass exchangers with steam-gas mixtures at subatmospheric pressures", Int'l Journal of Heat and Mass Transfer, v. 39, n. 5, pp. 965 - 973.

This paper outlines an experiment using direct-contact heat exchangers at subatmospheric pressures. The cycle uses both co- and countercurrent heat exchangers. The heat exchangers utilize metal packing to increase the surface area. The specific surface area used in this system was approximately $250 \mathrm{~m}^{2} / \mathrm{m}^{3}$. Various methods of filtration are used to remove noncondensables such as nitrogen and oxygen, as well as to remove mist prior to the compressor inlet. These heat exchangers are used both as heat exchangers and as intercoolers.

D’Orsi, N.C., Le Drew, B.A., Tsukasa, Y., Wight, S.E., “The efficiency limits of water vapor compressors", Concepts, ETI, Contract \#: 605-10010

This paper presents a thorough examination of turbomachine-based compression in the context of a vapor compression system using water as the refrigerant. Single- and multi-stage compression processes with both axial and centrifugal compressors are modeled and evaluated in terms of performance and size; no attempt was made to relate these parameters back to the economics of the cycle. At a much more cursory level, the use of both indirect and flash intercooling between compression stages was evaluated as a technique for improving performance and also in terms of the effect that the intercooling process would have on the compressor performance and size. Specific parameters and 
issues that were considered include: the implication of running multiple compressors on a common shaft, diffuser design parameters, compressor blade angles, and specific speed.

Goodheart, K.A., 2000, "Low firing temperature absorption chiller system", M.S. Thesis, University of Wisconsin - Madison.

This thesis evaluates the economics of low temperature absorption chillers. Absorption chillers use water as a refrigerant, albeit not in a vapor compression cycle. One component in the absorption chiller is an indirect-contact heat exchanger operating between the fluid streams that represent either the refrigeration load or the heat rejection medium and evaporating or condensing water. Therefore, this thesis provides a valuable benchmark for heat transfer coefficient correlations that are applicable to the indirect evaporators and condensers in a water-based vapor compression system. The thesis also contains useful information relative to cost analyses for many components that are common to both absorption and vapor compression chillers.

Kauffeld, M. Minds, G., Madsboll, H., 1996, "Water as Refrigerant", Danish Technological Institute, Refrigeration and Heat Pump Technology, Scanref, Leder.

The purpose of this paper is to describe the water vapor compression process currently used at the LEGO Systems plant. The refrigeration load at the plant is between $1300 \mathrm{~kW}$ and $1700 \mathrm{~kW}$, approximately half of the load that we are investigating. In this operation, the authors state that COPs in the range of $7-13$ have been observed. The compression is accomplished entirely using a two-stage centrifugal compressor. The 
compressor is fitted with thin titanium blades that, at low speeds are slightly swept and straighten at high speeds. The authors claim impeller tip speeds approaching $400 \mathrm{~m} / \mathrm{s}$, close to $80-90 \%$ of sound speed. The system is an open system with direct-contact heat exchangers. Therefore, de-aeration is mentioned as one of the gaseous filtrating processes. The specifics of this process or of other filtration processes are not described.

Knasiak, K.F., Schick, R.J., 2000, "Spray characterization for wet compression gas cooling applications", Proceedings of the $8^{\text {th }}$ Int'l Conference on Liquid Atomization and Spray Systems, Pasadena, CA.

This paper explores the idea of using atomizers as flash intercoolers in compression systems. The author describes the different types of atomizers and analyzes the quality of their spray characteristics. The experiments were done with air as the medium; however, the results can be applied to water vapor compression systems as well. Some of the information presented in this paper was used during the initial evaluation of spray-based direct-contact heat exchangers.

Kuhnl-Kinel, J., "New age water chillers with water as refrigerant.", CERN, Geneva, Switzerland.

This paper looks at the possibility of refrigerating water by employing flash evaporation made possible through the use of a large vacuum pump operating on water vapor. The cycle also required the use of a direct-contact heat exchanger for the 
condensation process. Very little specific information beyond the cycle concept was presented.

Liu, Z., Soedel, W., 1995, "A Mathematical Model for Simulating Liquid and Vapor Two-Phase Compression Processes and Investigating Slugging Problems in Compressors", HVAC\&R Research, v. 1, n. 2, pp. 99-109.

This paper presents a mathematical model of liquid slugging in reciprocating compressors. The authors find that there is a quality of suction vapor below which there is a high risk of liquid slugging that can lead to physical damage of the compressor. The paper provides an understanding of the relationship between the kinematics of reciprocating compressors and the tendency to slug.

Mitchley, S.R., Sheer, T.J., 1998, "Vacuum Boiling in a Water Vapour Refrigeration System”, Proceedings from Natural Working Fluids Conference '98, IIR, Oslo, Norway, pp. 53-61.

This paper presents an overview of some of the issues related to the design of a vacuum water vapor refrigeration system using a direct-contact heat exchanger for the purpose of a mine-shaft refrigeration system. An experimental set-up and procedure is described that allows visualization of vacuum phase change for water in a forced flow system consistent with the direct heat exchange components associated with a water vapor refrigeration system. 
One key point in this paper is that convection alone from a free surface is not very effective for heat exchange. According to the authors, "this means that the cooling of the bulk of the water is limited by the convection heat transfer rate once these temperature gradients have been established." In order to maintain the high heat transfer coefficient anticipated in a direct-contact heat exchanger, the authors assert that continuous violent boiling is necessary, because it is always exposing new water to the vacuum pressure and temperature gradients cannot be established. The authors indicate the need for flashing throughout the heat exchanger. Some of the results obtained with the direct-contact heat exchangers suggest that there was a substantial pressure drop over the length of the heat exchanger due to the high specific volume of the gas.

Mohtadi, M., Rao, D., 1983, "Novel direct-contact heat exchanger system for efficient heating of water", Proceedings of the ASME-JSME Thermal Engineering Joint Conference, Honolulu, v. 2, pp. 113-122.

This is a paper about direct-contact heat exchangers, where a gas is combusted in the cavitation region of cold water flow, in order to heat that water. Excellent heat transfer results were found and this is an example of high heat transfer characteristics in a gas and water in direct-contact heat exchanger system.

Mussulman, R., Warrington, R., 1983, "Analysis of a liquid/gas direct-contact heat exchanger concept", Journal of Energy, v. 7, n. 6, pp. 732-734. 
This paper is a summary of a model developed to represent both water cooling air and water warming air. One interesting result from this paper is the difference in ratio of ideal mass flow of air to the mass flow of water, depending on which fluid was heating the other. 


\section{CHAPTER 3 CYCLE SELECTION}

This chapter describes the cycle selection process. A number of vapor compression cycle configurations are analyzed using consistent, component-level performance metrics as opposed to the more detailed, geometry-based component level models that are described in Chapter 4. This approach allows the consideration and optimization of several cycles in order to determine the most attractive variation for detailed analysis. The vapor compression cycles considered varied, primarily, in the method of intercooling and included: non-intercooled, economized, flash intercooled, indirect intercooled, and direct intercooled. A liquid subcooled cycle is also analyzed as a variation of direct intercooling. These cycles and their predicted performance are described in detail in the following sections.

\subsection{Model Description}

In order to model the relatively complex cycle configurations associated with two- or more stages of intercooling, it is necessary to use modeling software capable of solving systems of simultaneous equations. The Engineering Equation Solver (EES) software has this ability along with the additional feature of a library of built-in thermophysical property functions for many fluids, including any refrigerant of interest, as well as the capability for providing a customizable, user-friendly graphical user interface. Therefore, EES was selected for all of the modeling described in this and later sections.

The first subsection describes the underlying component level performance assumptions used to carry out the comparison. The following subsections describe the 
non-intercooled, economized, flash intercooled, indirect intercooled, direct intercooled, and liquid subcooled refrigeration cycles.

\subsubsection{Assumptions}

A consistent set of component performance metrics and operating conditions are used to create a meaningful baseline for comparison among the refrigeration cycle configurations. All of the refrigeration cycles are sized to provide 1000 tons of refrigeration. The chilled water and the condenser water are supplied and returned at the temperatures specified by ARTI in Table 3.

Table 3 ARTI specified water temperatures.

\begin{tabular}{|r|r|r|c|}
\hline \multirow{2}{*}{ Evaporator } & Chilled Water Supply Temperature & $44^{\circ} \mathrm{F}$ & \multirow{2}{*}{$\Delta \mathrm{T}_{\text {chw }}=-10^{\circ} \mathrm{F}$} \\
\cline { 2 - 3 } & Chilled Water Return Temperature & $54^{\circ} \mathrm{F}$ & \\
\hline \multirow{2}{*}{ Condenser } & Condenser Water Supply Temperature & $95^{\circ} \mathrm{F}$ & \multirow{2}{*}{$\Delta \mathrm{T}_{\mathrm{cw}}=+10^{\circ} \mathrm{F}$} \\
\cline { 2 - 3 } & Condenser Water Return Temperature & $85^{\circ} \mathrm{F}$ & \\
\hline
\end{tabular}

In this part of the analysis, the evaporator and condenser are assumed to have no pressure drop and the approach temperature difference is assumed to be zero for all condensers and $0.5^{\circ} \mathrm{F}$ for all evaporators. The polytropic efficiency of all compressors is set at $70 \%$.

It is important to note here that the compressor polytropic efficiency is different from the traditional isentropic efficiency commonly used in simplified thermodynamic cycle analyses. The isentropic efficiency of a compressor is, inherently, dependent on the pressure ratio at which it is operating. The polytropic efficiency is an engineering tool that can be used to compare multistage compression processes without confounding differences in performance by the differences in isentropic efficiency that occurs as the pressure ratio across each stage varies. That is, a different amount of power is required to 
achieve a given pressure ratio if two stages are used rather than one stage even if the same isentropic efficiency is assumed for each individual compressor. This behavior can mask true variations in the cycle coefficient of performance related to multi-staging.

The polytropic efficiency is defined as being equal to the isentropic efficiency at an infinitesimally small pressure ratio. When applied to a finite pressure ratio, a compressor with a given polytropic efficiency will have a lower isentropic efficiency. This is illustrated in Figure 3 on a temperature-entropy diagram in which the compression process for two compressors, one with an isentropic efficiency of 0.70 and the other with a polytropic efficiency of 0.70 , are overlaid on the same plot. Notice that the compressor with the polytropic efficiency of 0.70 requires more work than the one with an isentropic efficiency of 0.70 . This effect is related to the divergence of the isobars on a T-s diagram; the isentropic power gets larger as one moves toward higher entropy.

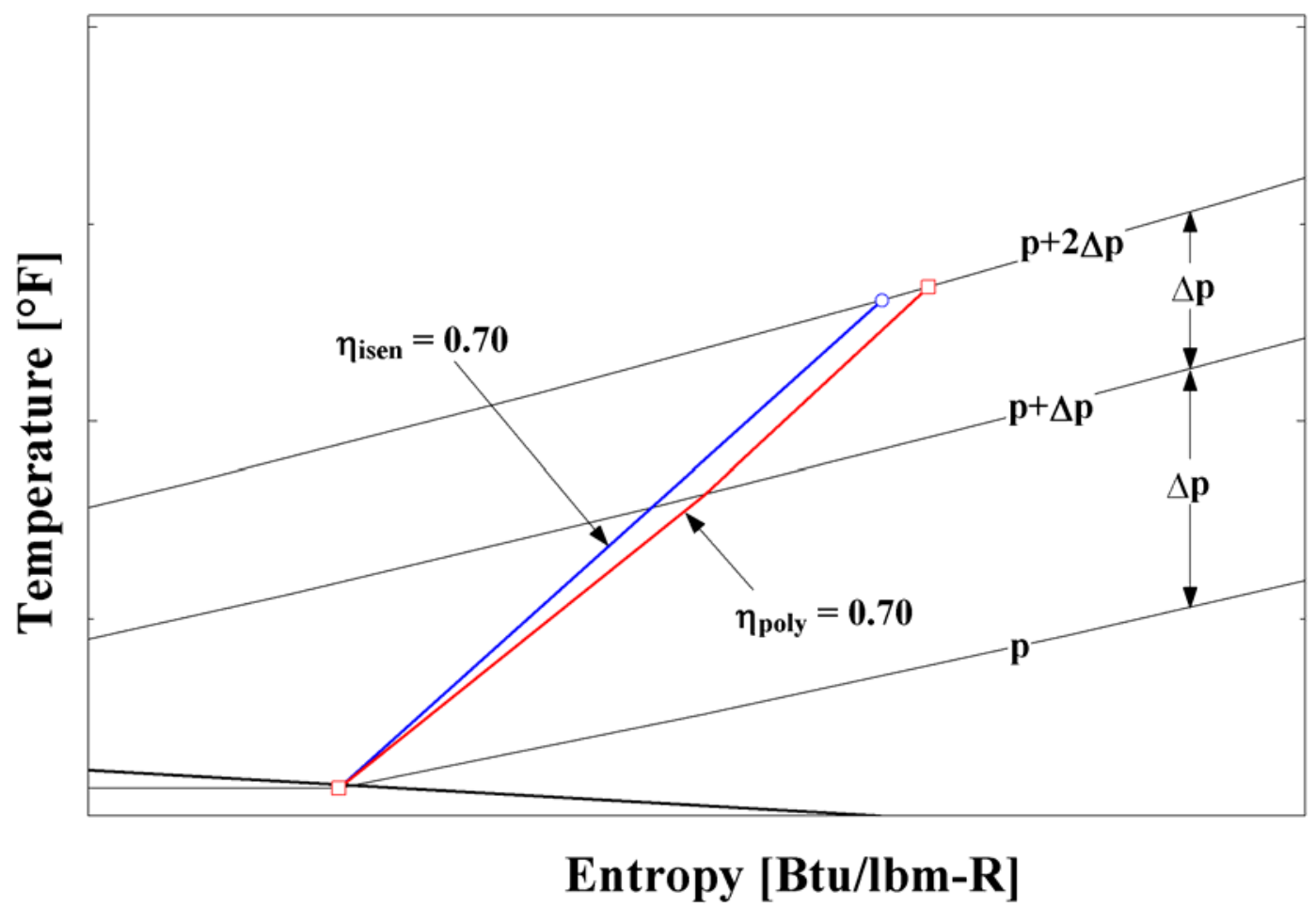

Figure 3 T-s diagram illustrating difference between isentropic and polytropic efficiency. 
The advantage of using polytropic efficiency in our calculations is that it makes one compressor operating at a given polytropic efficiency across a large pressure ratio compare equitably with two compressors operating between the same pressure ratio but in two stages. Figure 6, for example, illustrates the different results obtained when using isentropic and polytropic efficiency to predict the COP of a multi-stage refrigeration cycle. To convert from polytropic efficiency to isentropic efficiency, the pressure difference between the suction and discharge of each compressor must be broken into many very small pressure differences $(\Delta \mathrm{p})$. The polytropic efficiency is then applied to a compressor running between these infinitely small pressure differences. The isentropic efficiency is then determined using the following equation.

$$
\eta_{\text {isen }}=\frac{\mathrm{h}_{2 \mathrm{~s}}-\mathrm{h}_{1}}{\mathrm{~h}_{\text {exit }}-\mathrm{h}_{1}}
$$

where $\mathrm{h}_{\text {exit }}$ is defined as the theoretical discharge enthalpy obtained from the integration process, $\mathrm{h}_{2 \mathrm{~s}}$ is the discharge enthalpy for an adiabatic and reversible compressor operating with the same discharge pressure, and $\mathrm{h}_{1}$ is the suction enthalpy.

\subsubsection{Non-Intercooled Cycle}

An EES model of a non-intercooled cycle with an arbitrary number of compression stages is shown in Figure 4 with two compression stages. Figure 5 shows the thermodynamic cycle on a T-s diagram. 


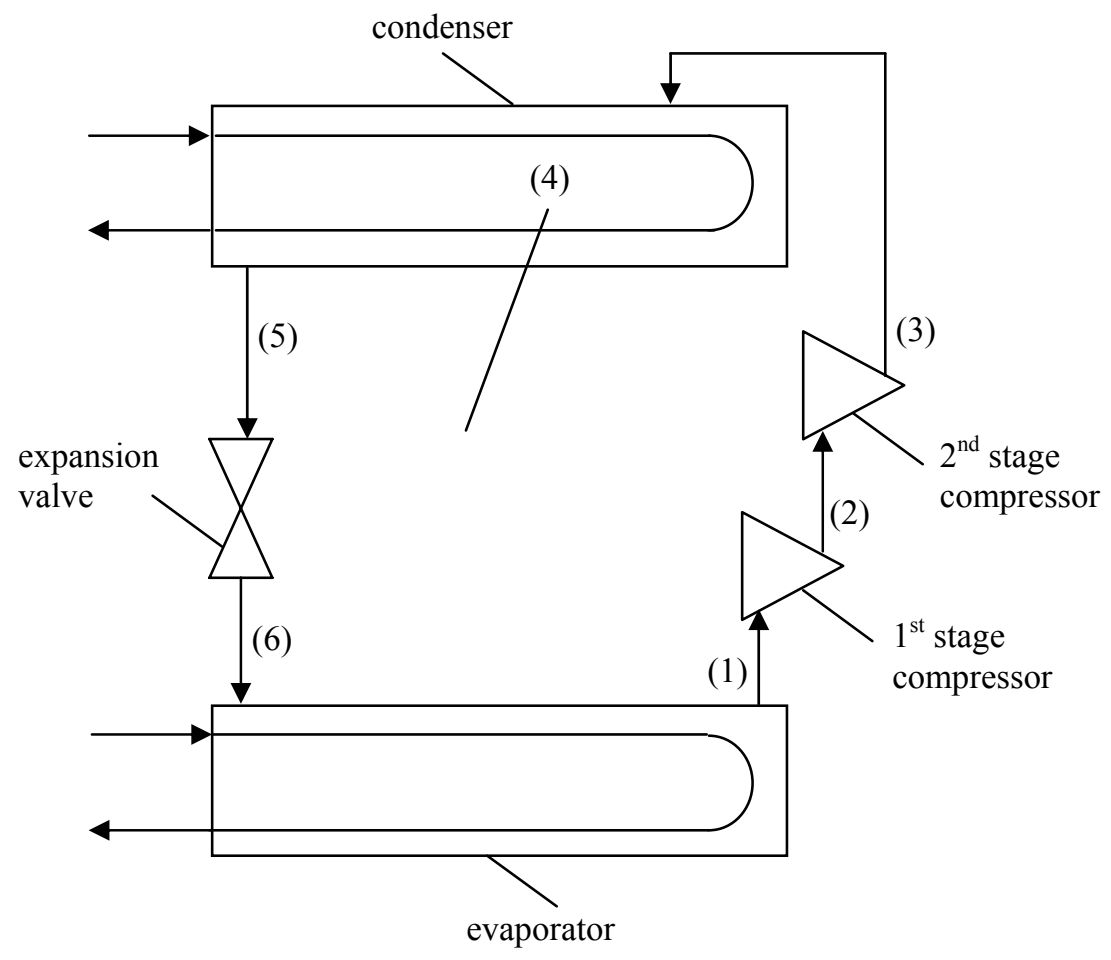

Figure 4 Cycle schematic for two-stage cycle with no intercooling.

* Note: state (4) is included for clarity relative to the T-s diagram shown in Figure 5.

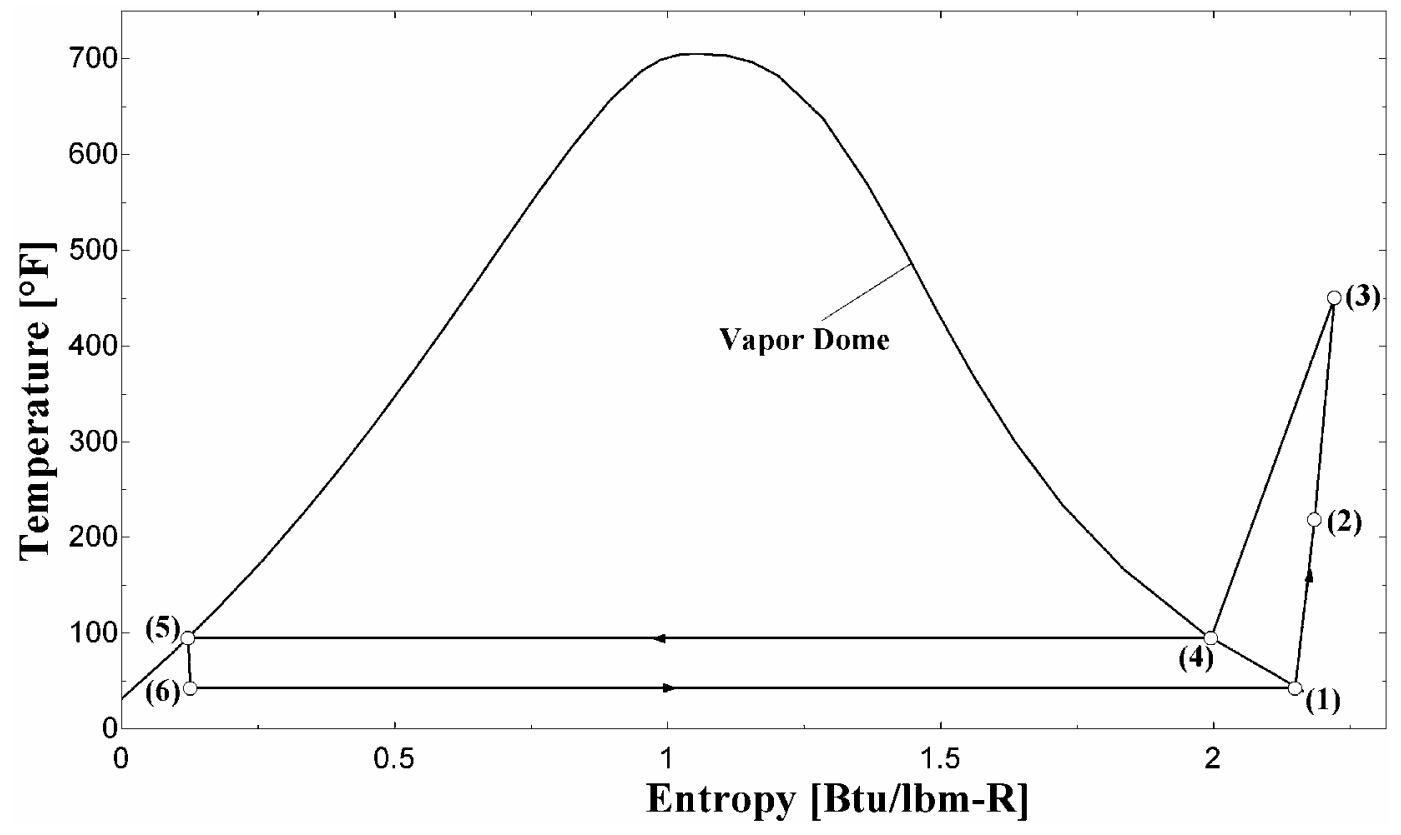

Figure 5 Temperature-entropy diagram of a two-stage cycle with no intercooling. 
The individual stage pressure ratios can be specified in a number of ways. The two techniques supported by our model are: (1) equal isentropic work done per stage $\left(\Delta \mathrm{h}_{1 \mathrm{~s}}=\Delta \mathrm{h}_{2 \mathrm{~s}}\right)$, and (2) equal pressure ratio $\left(\mathrm{r}_{\mathrm{c}}\right)$ per stage ${ }^{2}$. With no intercooling, the cycle performance is insensitive to the distribution of the overall pressure ratio between the various stages due to the use of the compressor polytropic efficiency.

Once the pressures at each state point are set, the model determines all of the properties for each of the state points. The saturated vapor properties at the compressor inlet are determined using the ARTI-specified chilled water temperature minus the specified evaporator approach temperature difference. The exit properties for each stage of compression can be obtained using the compressor efficiency and pressure ratios. The system high-side pressure is established by the ARTI-specified condenser water temperature and the assumed condenser approach temperature difference. In the condenser, the refrigerant vapor must first be desuperheated along a line of constant pressure to the vapor dome and then condensed to a saturated liquid. The expansion process is assumed to be isenthalpic. No refrigerant-side pressure drops are accounted for in the condenser or the evaporator in this simplified model. After defining all of the states on a T-s diagram, it is possible to calculate the coefficient of performance (COP) of the cycle, determine the mass flow rate through each component, and calculate other quantities of interest such as the compressor inlet volumetric flow rate.

Figure 6 shows the variation of the COP with the number of compression stages using the assumptions described in Section 3.1.1 for a non-intercooled cycle using both a constant isentropic and polytropic compressor efficiency per stage. As a baseline, the

\footnotetext{
${ }^{2} r_{c}=\sqrt[N S C]{\frac{p_{\text {condenser }}}{p_{\text {evaporator }}}}$ where NSC is the number of stages of compression.
} 
water cycle is compared with an R-134a cycle (assuming a polytropic compressor efficiency of $70 \%$ ). When isentropic efficiency is used, the efficiency decreases with increasing stages of compression, eventually approaching the polytropic efficiency line.

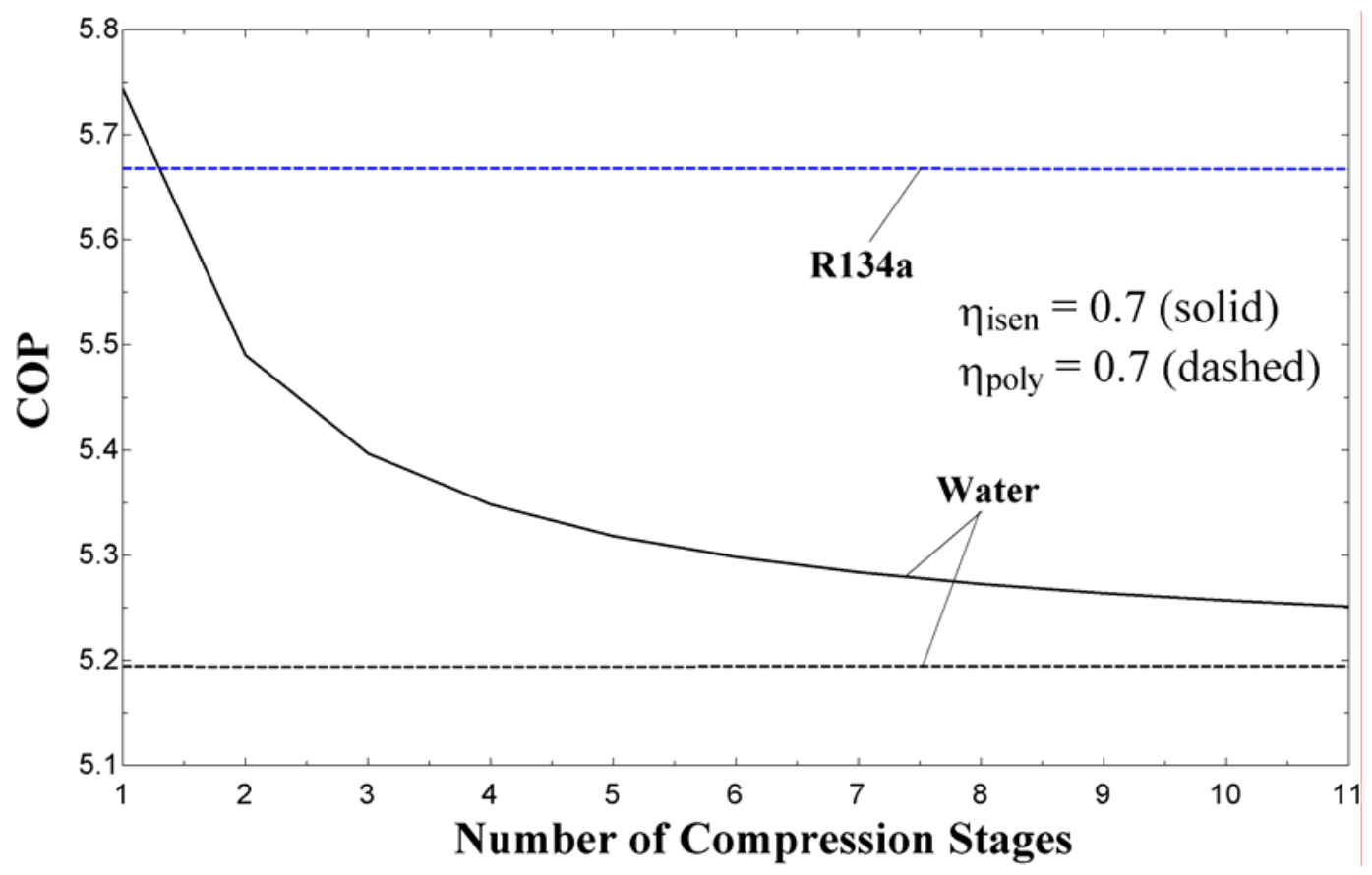

Figure 6 Comparison of the cycle COP as a function of the number of compression stages using both polytropic and isentropic compression efficiencies for both water and $\mathrm{R}-134 \mathrm{a}$ as a refrigerant in a non-intercooled cycle.

\subsubsection{Economized Cycle}

An economizer is one type of intercooling configuration that can be used between compression stages in order to lower the superheat irreversibilities that otherwise dominate water-based refrigeration. Multi-stage expansion is used and the pressures at the exit of each expansion stage are set to the intermediate pressures of the compression stages. The 2-phase fluid leaving each expansion valve is separated by phase: the vapor is mixed with the compressor exit stream (superheated steam) in order to provide some cooling while the cool liquid is passed on to the next expansion valve in series. 
A cycle schematic of a 2-stage, economized cycle is shown in Figure 7.

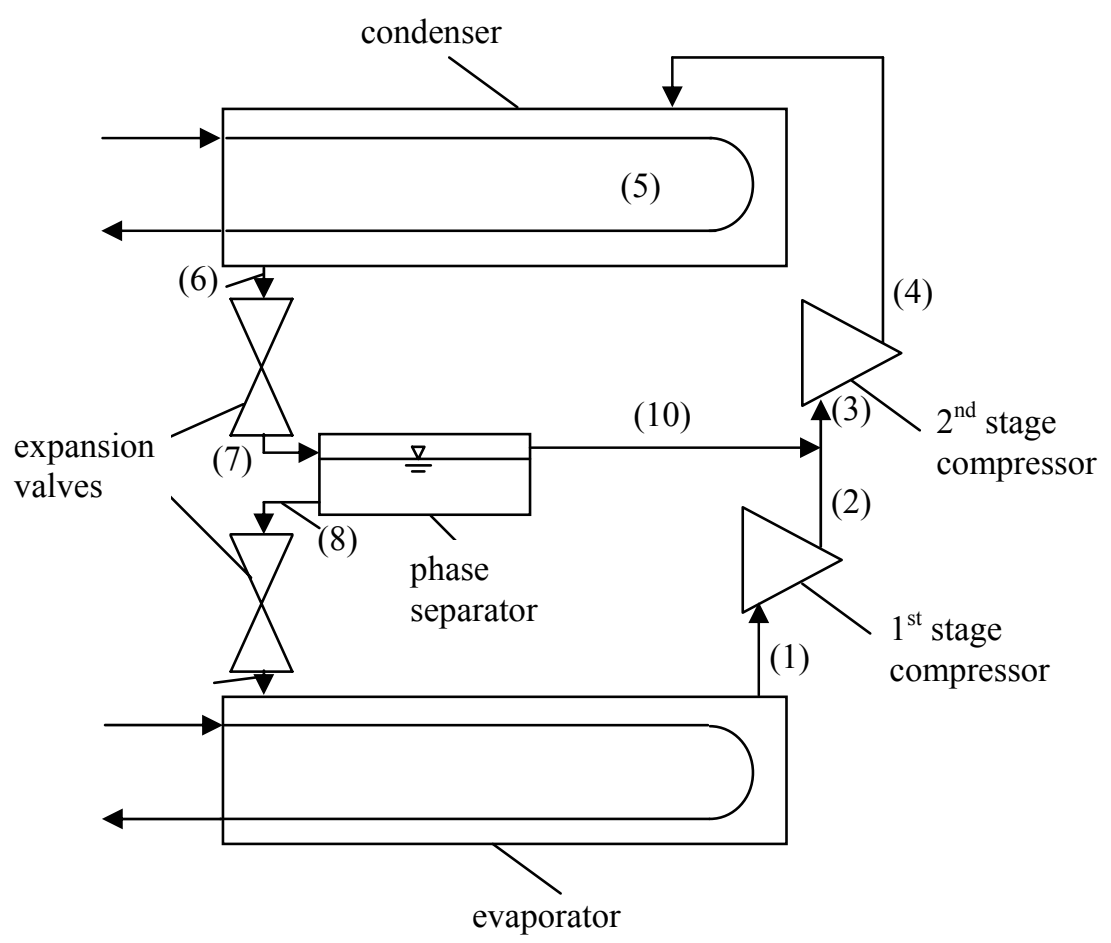

Figure 7 Schematic of a two-stage economized cycle.

Figure 8 illustrates the cycle on a T-s diagram. Figure 9 illustrates an exploded view of the $1^{\text {st }}$ stage compressor discharge and $2^{\text {nd }}$ stage compressor suction states. These states lie on an isobar but are separated slightly in temperature due to the addition of the vapor; the temperature difference between these states reflects the influence of intercooling. Note the relatively small change in temperature $\left(\Delta \mathrm{T}_{\text {econ }} \approx 2{ }^{\circ} \mathrm{F}\right)$, indicating a relatively small intercooling effect. Flash gas after an expansion valve is the result of irreversibilities in the expansion process. A refrigerant like R-22 or R-134a have large throttling losses and therefore will have more flash gas than a similar water cycle. Economizing is therefore a more effective cycle configuration for those typical refrigerants. On the other hand, a water cycle has relatively low throttling losses and this makes the economized cycle a poor choice. 


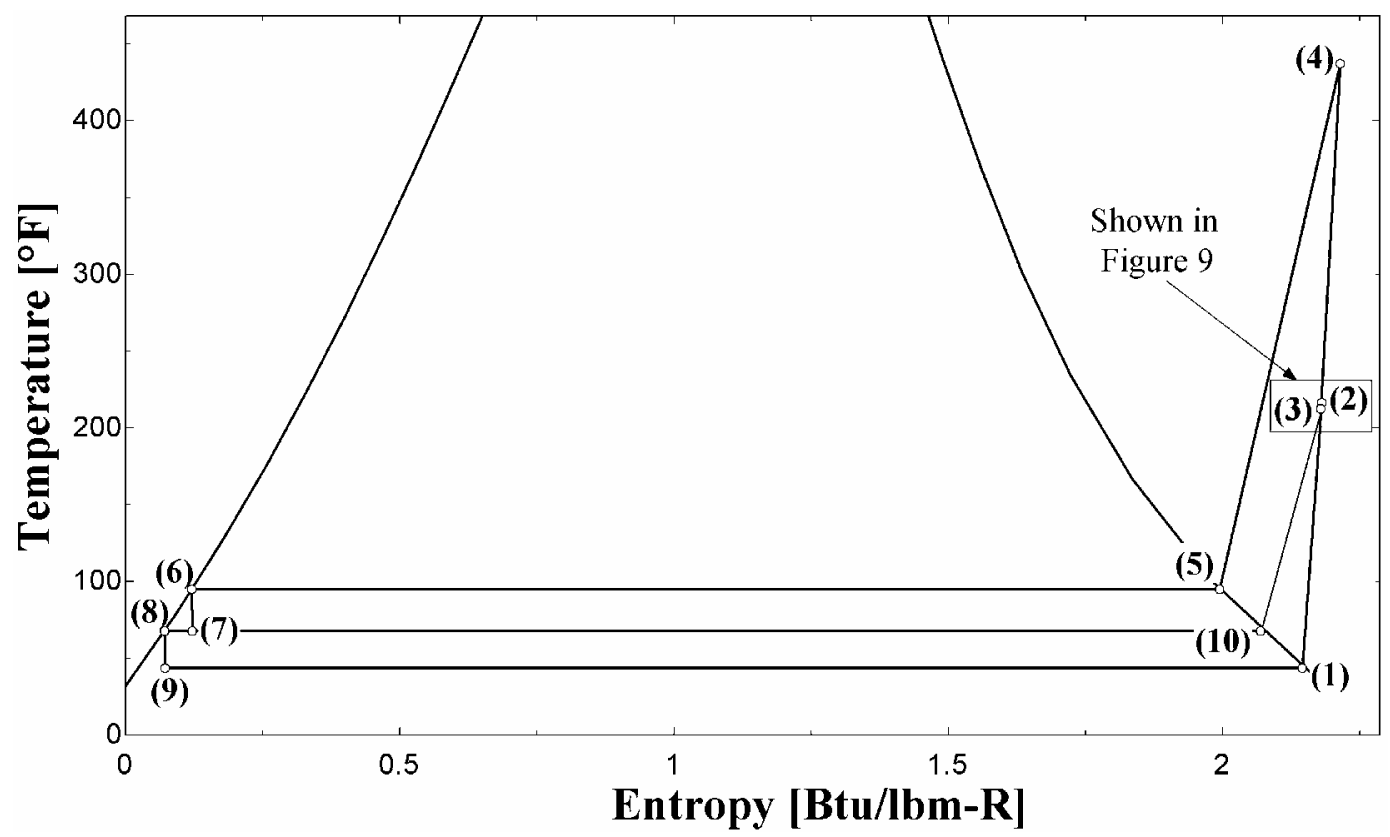

Figure 8 Schematic of a two-stage economized cycle.

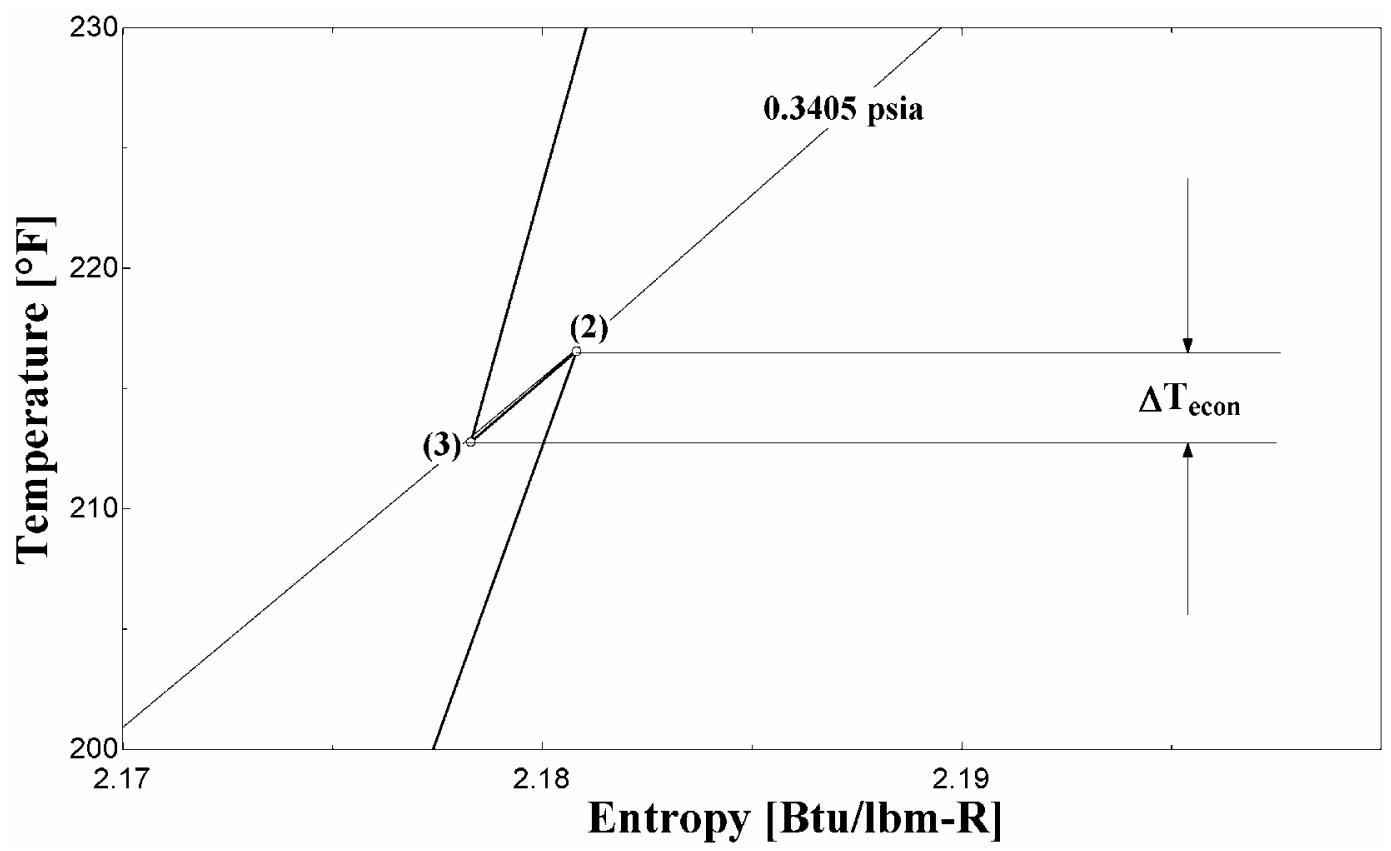

Figure 9 Expanded view of $1^{\text {st }}$ stage compressor discharge $/ 2^{\text {nd }}$ stage compressor suction states.

The pressures at each state point are initially set based on the condenser and evaporator temperatures and the stage pressure ratios. With pressures assigned (again based on either equal stage pressure ratios or equal isentropic enthalpy rise), the inlet and 
exit conditions for each of the expansion valves can be determined. The compressor inlet and exit properties are determined, progressing from the suction side of the first stage compressor through each subsequent compression process. Saturated vapor is assumed to enter the first stage compressor. The compressor exit state is related only to the specified polytropic efficiency and pressure ratio. The inlet to the next compressor (stage 2) is obtained from an energy and mass balance that captures the mixing process associated with the vapor obtained from the economizer. This process continues until all compressor inlet and exit states are defined. The model computes the cycle COP and other parameters of interest.

Figure 10 illustrates the calculated COP for the economized cycle. Also shown in Figure 10 are the previous results for the non-intercooled cycle for comparison. Notice that the increase in the cycle performance associated with economizing is insignificant; consequently, this cycle option can be eliminated from further consideration due to the small marginal benefit that accompanies the more complex system. 


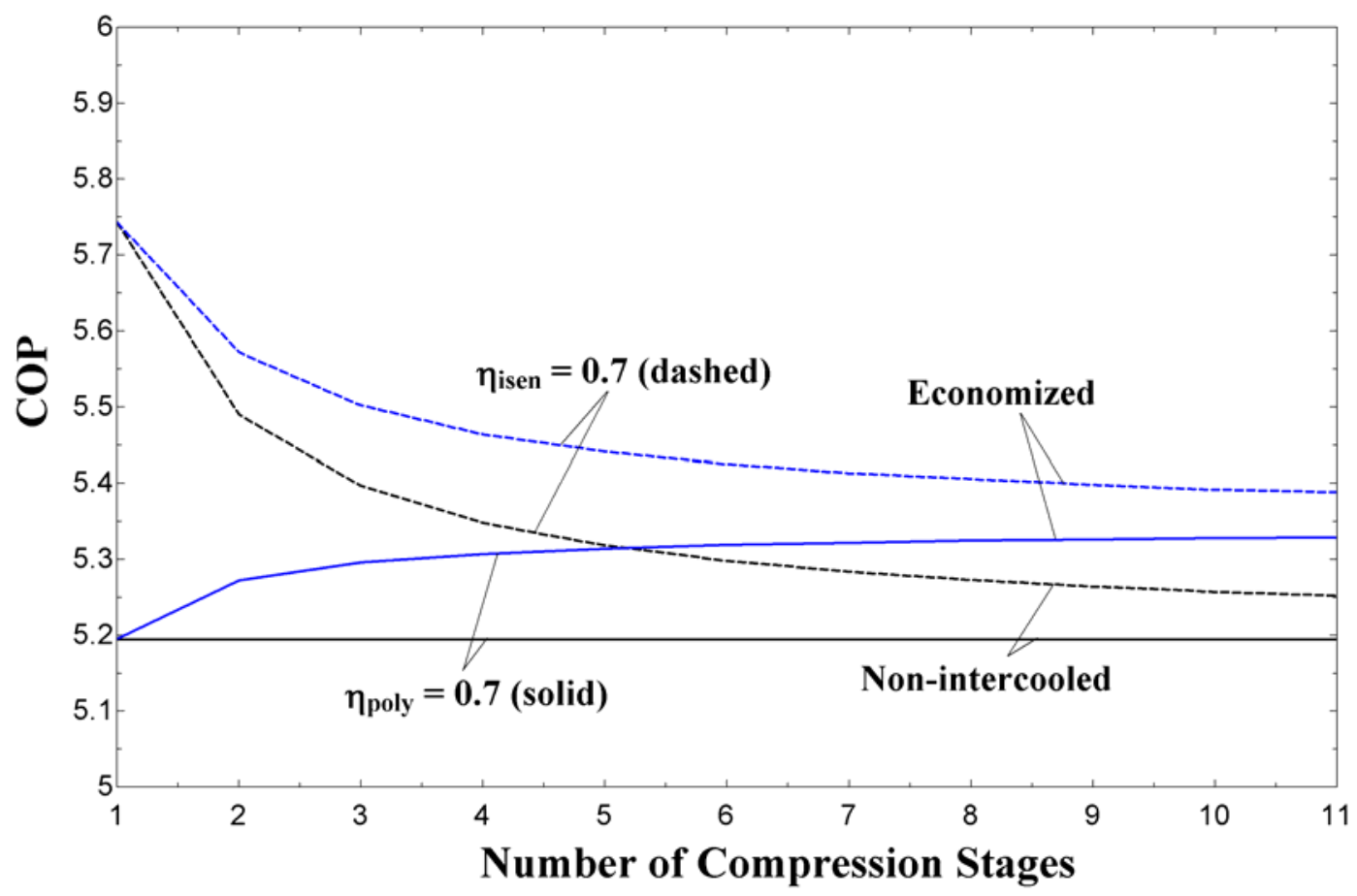

Figure 10 Cycle COP as a function of the number of compression stages for economized and non-intercooled cycles for water as a refrigerant.

Figure 11 is a comparison of water and R-134a as a refrigerant in both nonintercooled and economized cycles. The compressors are all assumed to have a polytropic efficiency of $70 \%$. Notice the much larger increase in efficiency associated with the R-134a cycle. Because there is more irreversibility in the throttling process in an R-134a cycle resulting in more flash gas, the desuperheating process is augmented and the quality of the saturated liquid vapor mixture entering the evaporator is increased. 


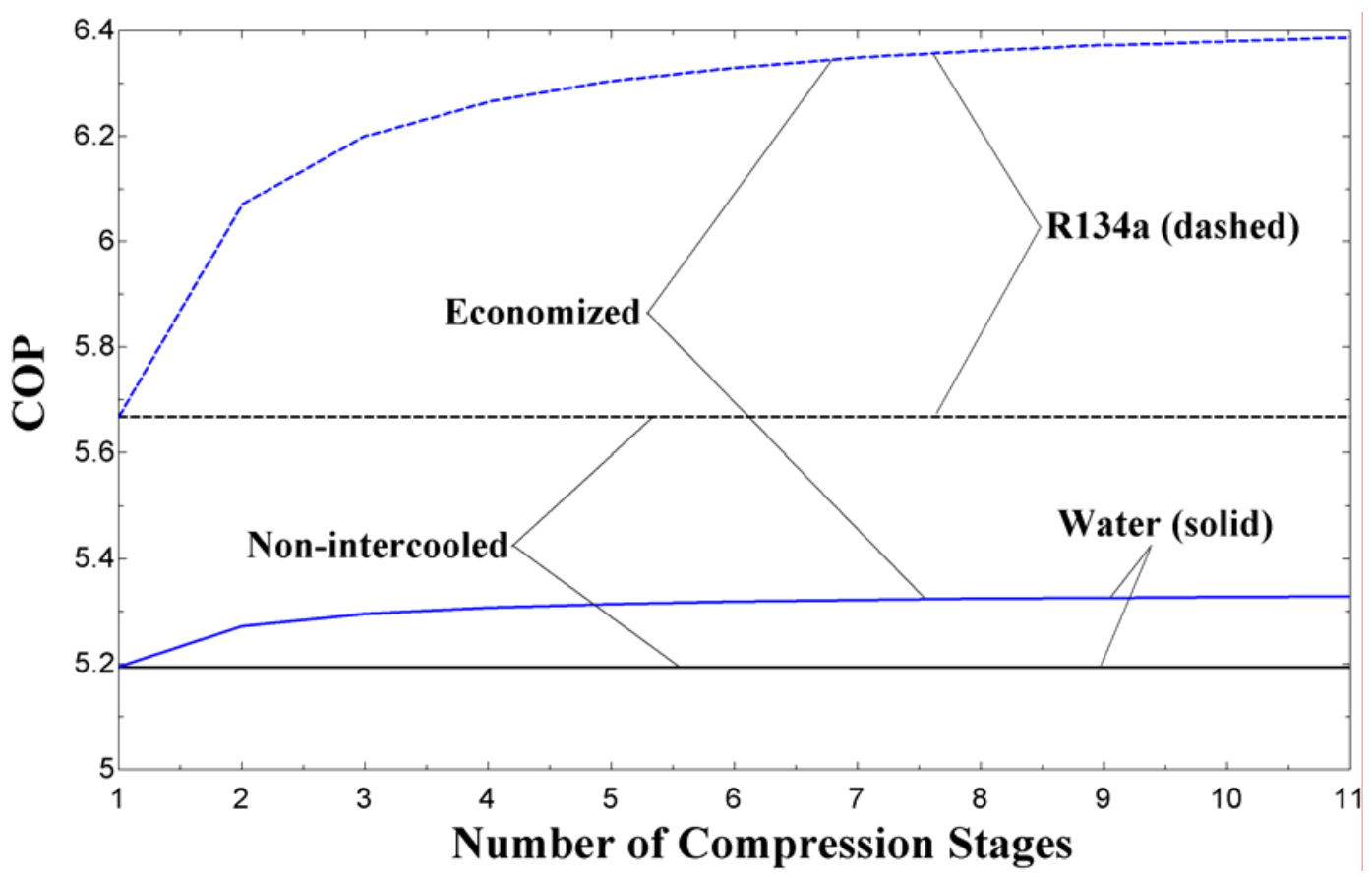

Figure 11 Cycle COP as a function of the number of compression stages for economized and non-intercooled cycles for both water and R-134a as a refrigerant.

\subsubsection{Indirect Intercooled Cycle}

In an indirect intercooled cycle, the vapor leaving the lower pressure compressor stages can be cooled to near the condenser water temperature (with some approach temperature). Instead of utilizing the latent heat of the condenser water by evaporating it, the heat rejection results in a sensible energy gain by the condenser water. The result is that a significant amount of condenser water must be used in order to affect any significant intercooling on the vapor; the benefit of this configuration is that there is no mass addition to the downstream compressors. However, the temperature of the refrigerant vapor leaving each compressor can only be reduced to the condenser water inlet temperature rather than all the way to the vapor dome as in the flash intercooling configuration, described in a subsequent section. Also, the indirect intercooler heat 
exchanger presents an additional pressure drop between compression stages (pressure drop is neglected in this simplified analysis but will be a design concern in an actual system built using this arrangement). Finally, there will be significant capital costs associated with the indirect intercooling heat exchangers. Figure 12 is a T-s diagram for a two-stage indirect intercooled cycle. Figure 13 is a schematic of this two-stage cycle.

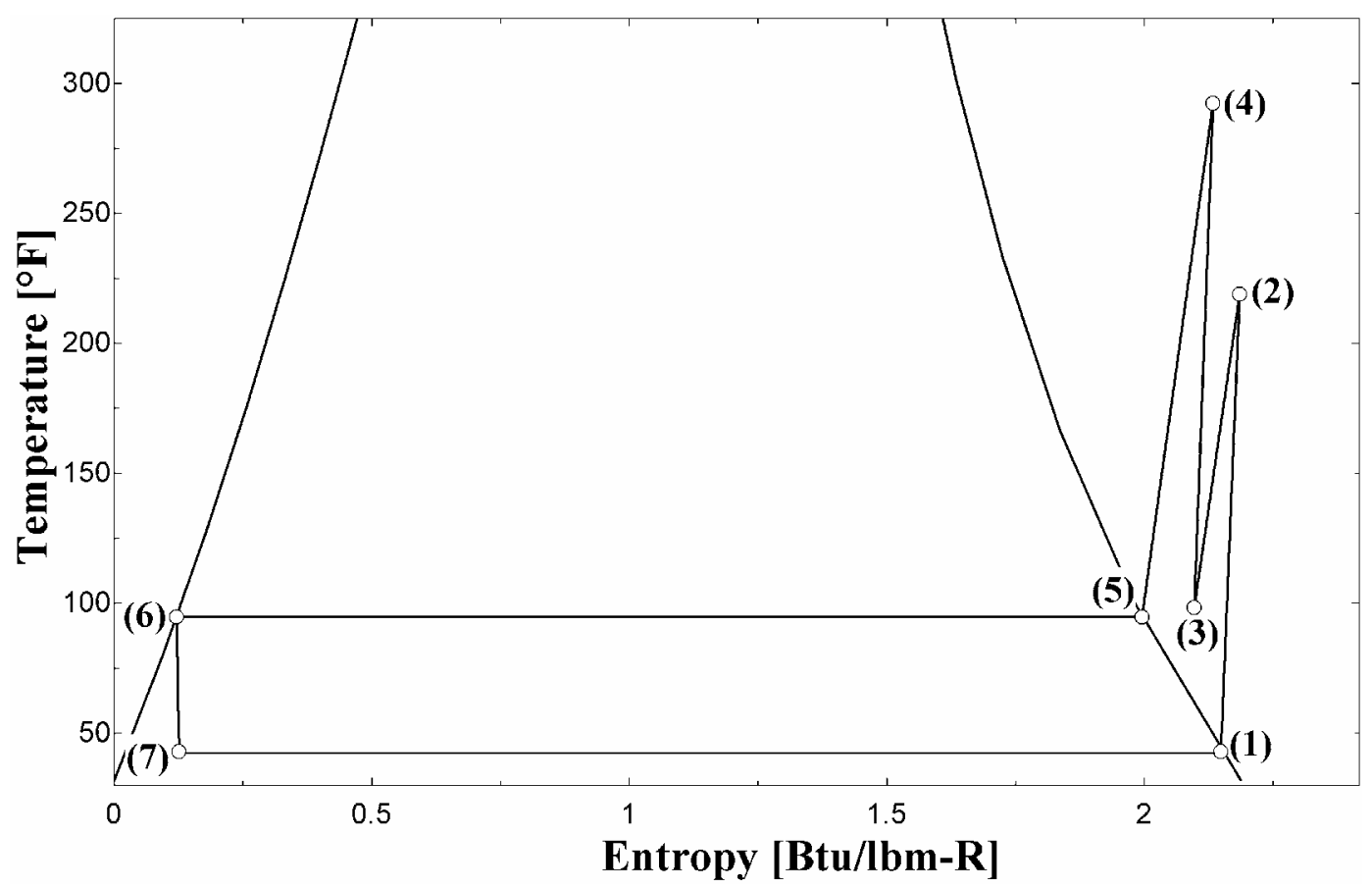

Figure $12 \mathrm{~T}$-s diagram of an indirect intercooled cycle. 


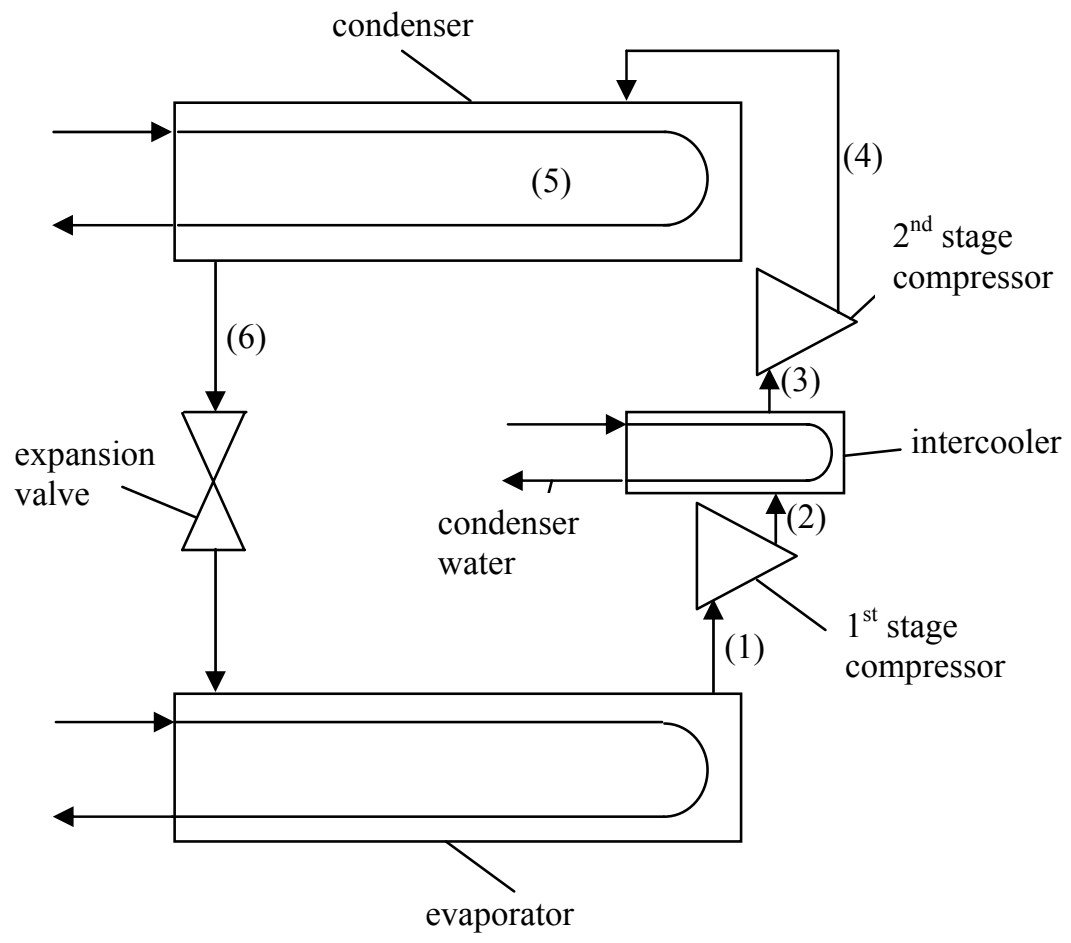

Figure 13 Schematic of a two-stage indirect intercooled cycle.

Figure 14 shows the predicted variation of the cycle COP with the number of stages of compression for an indirect intercooled cycle as well as the previouslypresented refrigeration cycle configurations (non-intercooled and economized). Results are shown both for constant isentropic and polytropic compressor efficiency. Note the significant performance increase associated with the indirect intercooled cycle. 


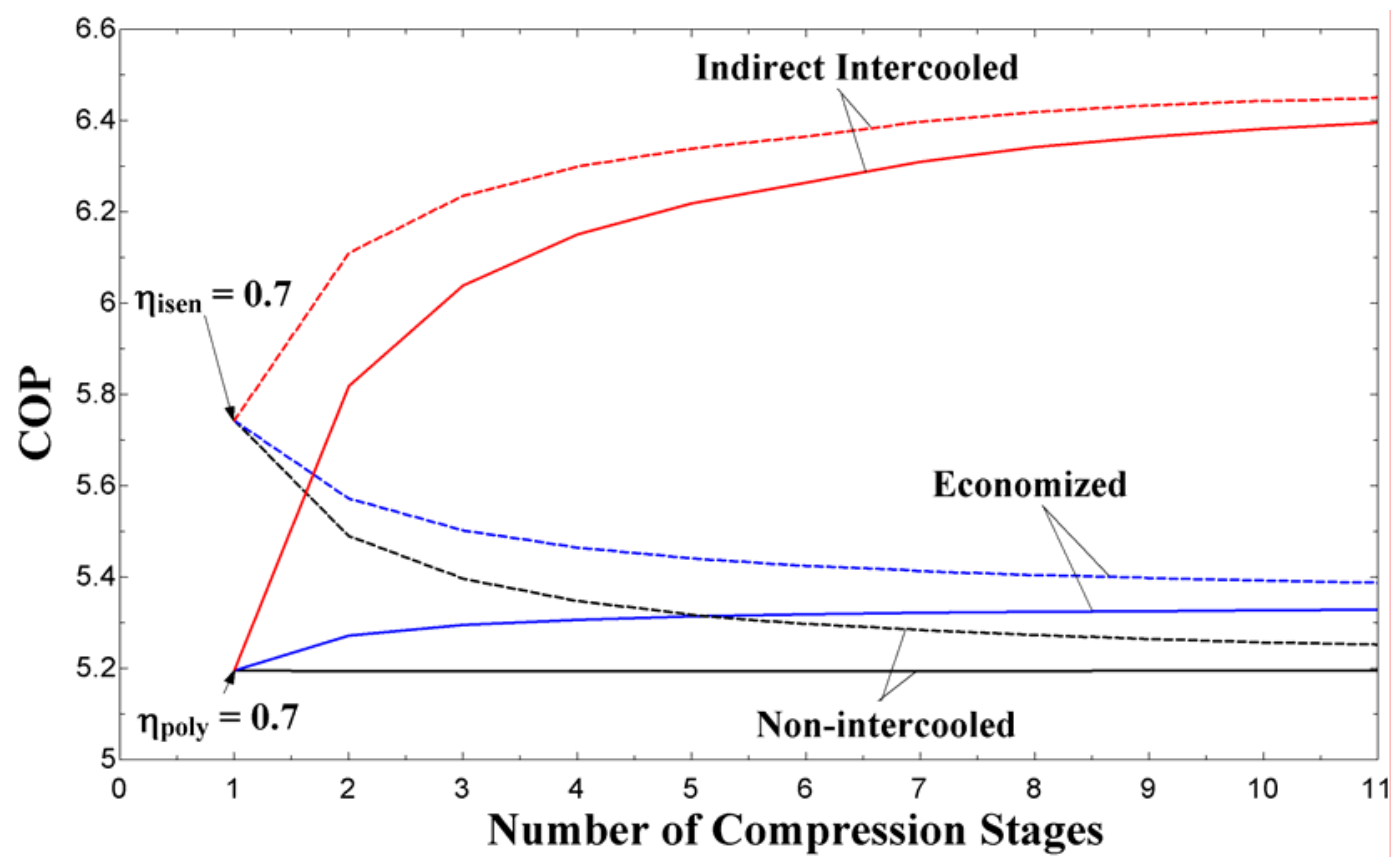

Figure 14 Cycle COP as a function of the number of stages of compression for the indirect intercooled cycle (as well as the non-intercooled and economized cycles).

Figure 15 overlays the polytropic efficiency curves from Figure 14 onto the similar curves for an R134a cycle. A curve associated with indirect intercooling is not added to Figure 15 because it is impossible to utilize indirect intercooling for R-134a as there is not enough superheat generated in the compression process to allow intercooling in this configuration where the intercooling temperature is limited by the condenser water temperature. The exit temperatures of the intermediate stage compressor exits do not reach the condenser water temperature. 


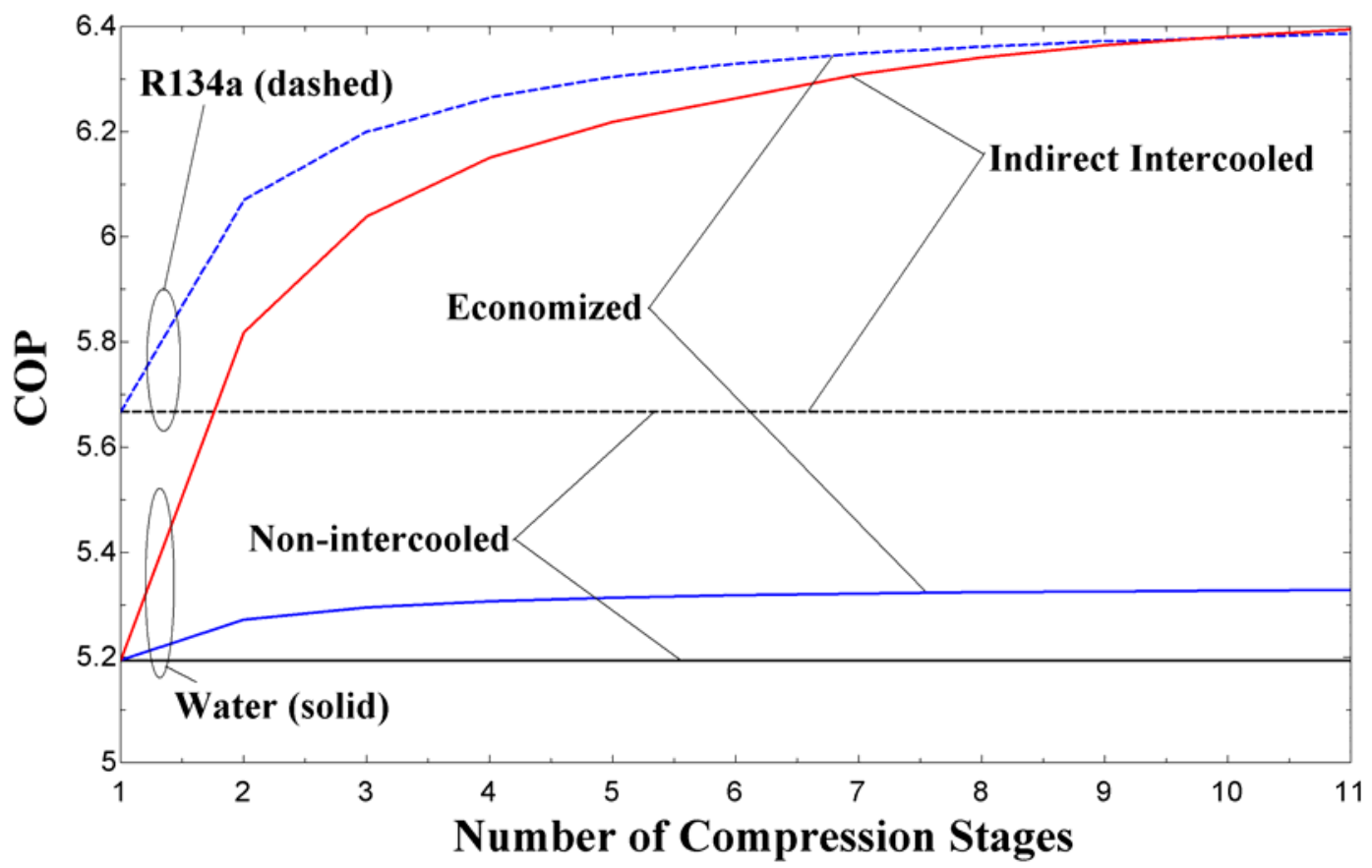

Figure 15 Cycle COP as a function of the number of compression stages for indirect intercooled, economized, and non-intercooled cycles for both water and R-134a as a refrigerant.

\subsubsection{Flash Intercooled Cycle}

Flash intercooling is another technique for reducing the superheat that is generated in the compression process. There are two alternative configurations that can be used to provide flash intercooling. Water at the condenser exit can be expanded to the compressor intermediate pressure and subsequently injected as a fine spray into the compressor exit stream, vaporizing and absorbing discharge superheat. This configuration is referred to as "Process Water Bleed Off" (PWB). A variation on this design is a second technique that involves using an open cycle in which a portion of the condenser water is throttled to the intermediate pressure and injected as a fine spray, performing the same task that the condenser exit stream does in the PWB cycle configuration. If this method is used, then the added mass flow from the outside source 
must be removed at some other point in the cycle requiring a pump. The open cycle also introduces potential filtration issues (purging noncondensables, particle and chemical contaminant removal, etc.) that are not explored in this idealized cycle. This type of flash intercooling is referred to as the "Condenser Water Bleed Off" configuration (CWB).

The two configurations are illustrated schematically in Figures 16 and 17. The T-s diagram for the PWB configuration is illustrated in Figure 18. The effect of intercooling is magnified in Figure 19 and shows clearly that the flash intercooling is much more effective than the economizer approach, previously shown in Figure 9.

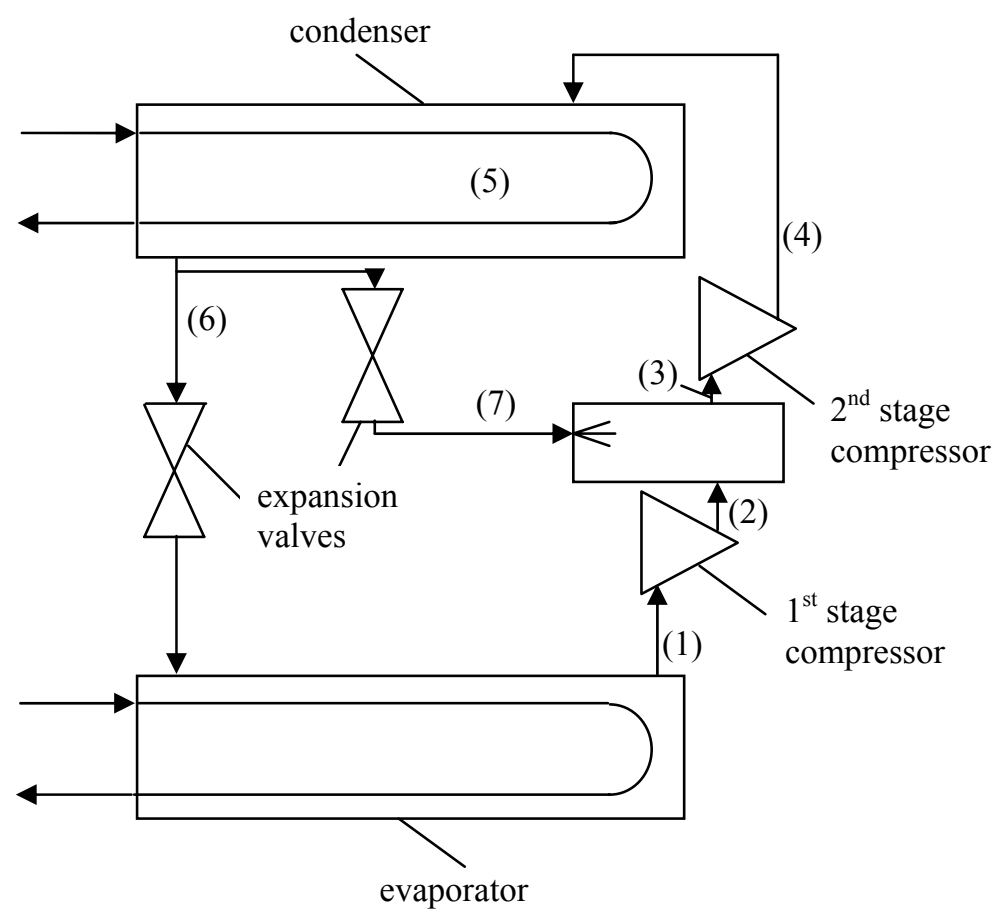

Figure 16 Schematic of a 2-stage flash intercooled cycle ("Process Water Bleed Off" configuration). 


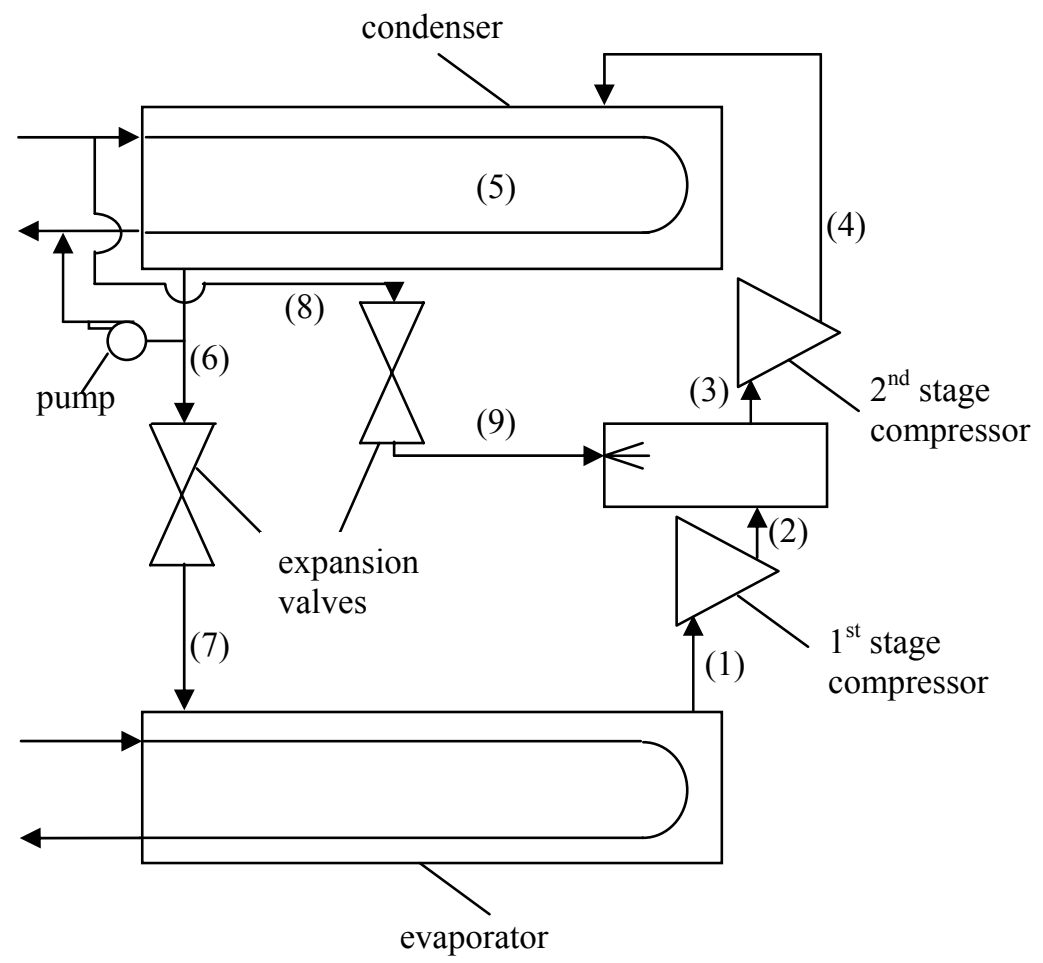

Figure 17 Schematic of a 2-stage flash intercooled cycle ("Condenser Water Bleed-off" configuration).

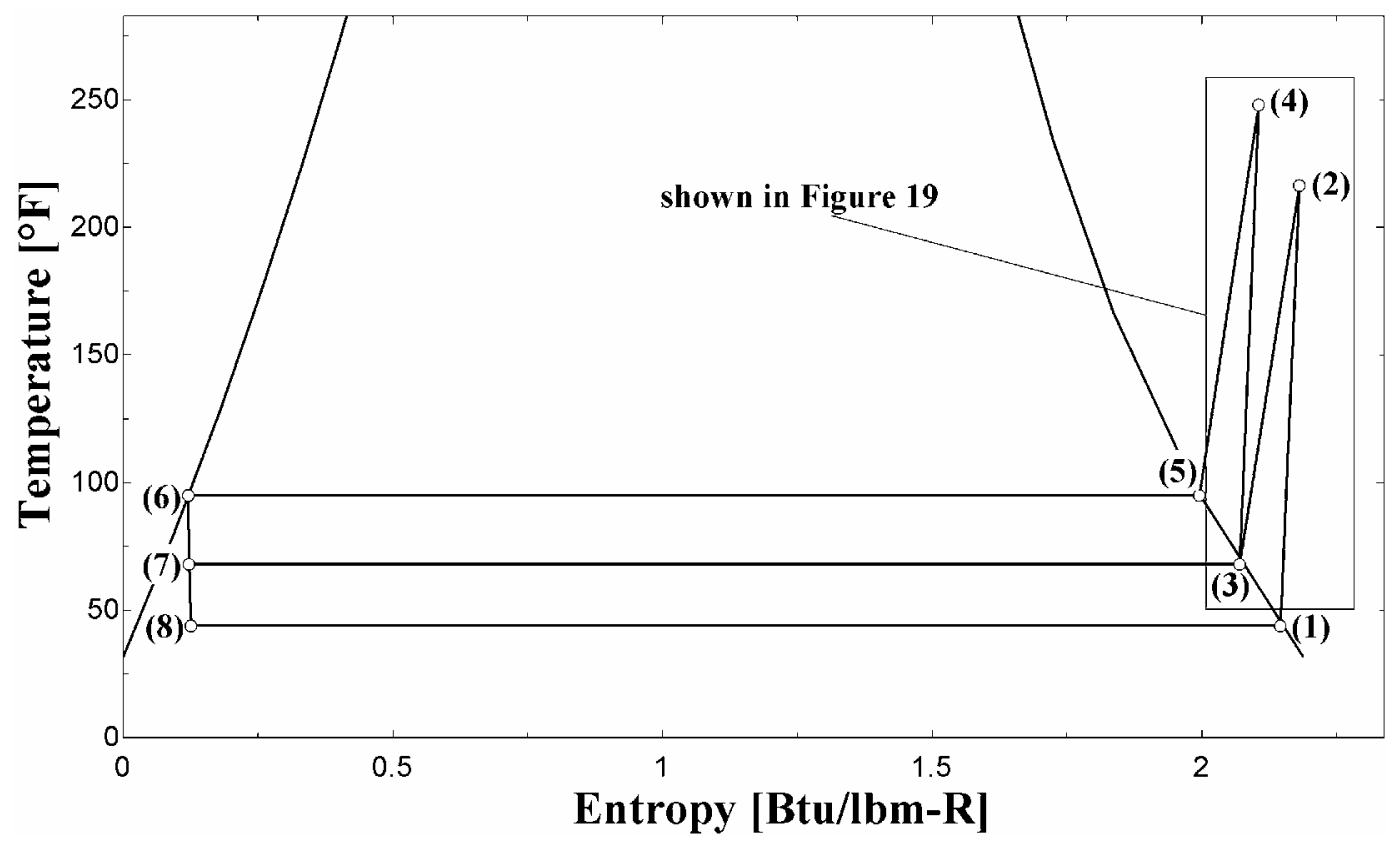

Figure 18 T-s diagram of a 2-stage flash intercooled cycle ("Process Water Bleed-off" configuration). 


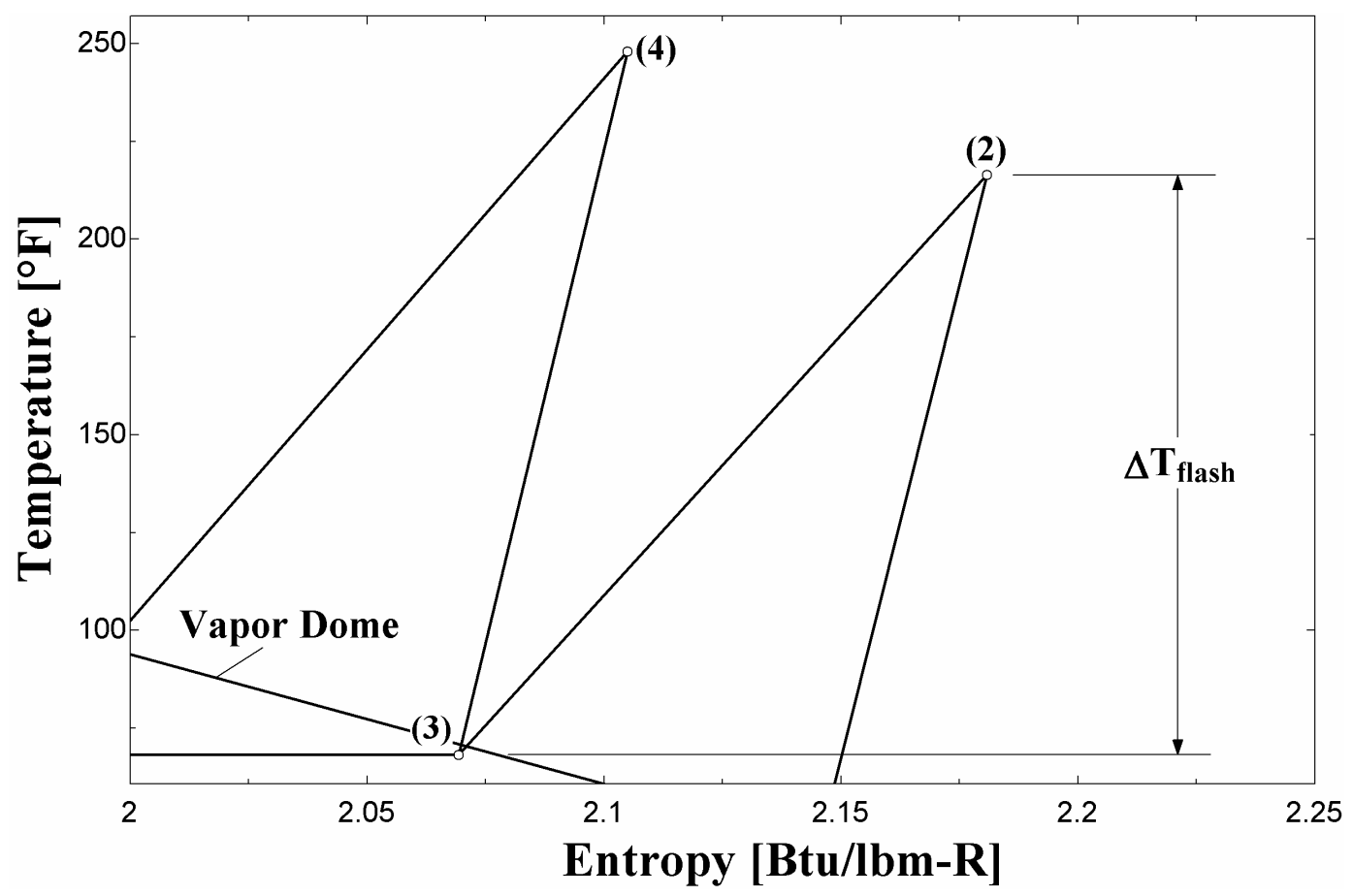

Figure 19 Expanded View of the 1st stage compressor discharge (2)/2nd stage compressor suction (3) states for the flash intercooled cycle.

Figure 20 illustrates the cycle COP as a function of the number of compression stages for a flash intercooled cycle in the CWB configuration. Both economized and flash intercooled cycles perform significantly better than the non-intercooled cycle. However, the flash intercooled cycle shows a somewhat larger performance benefit. 


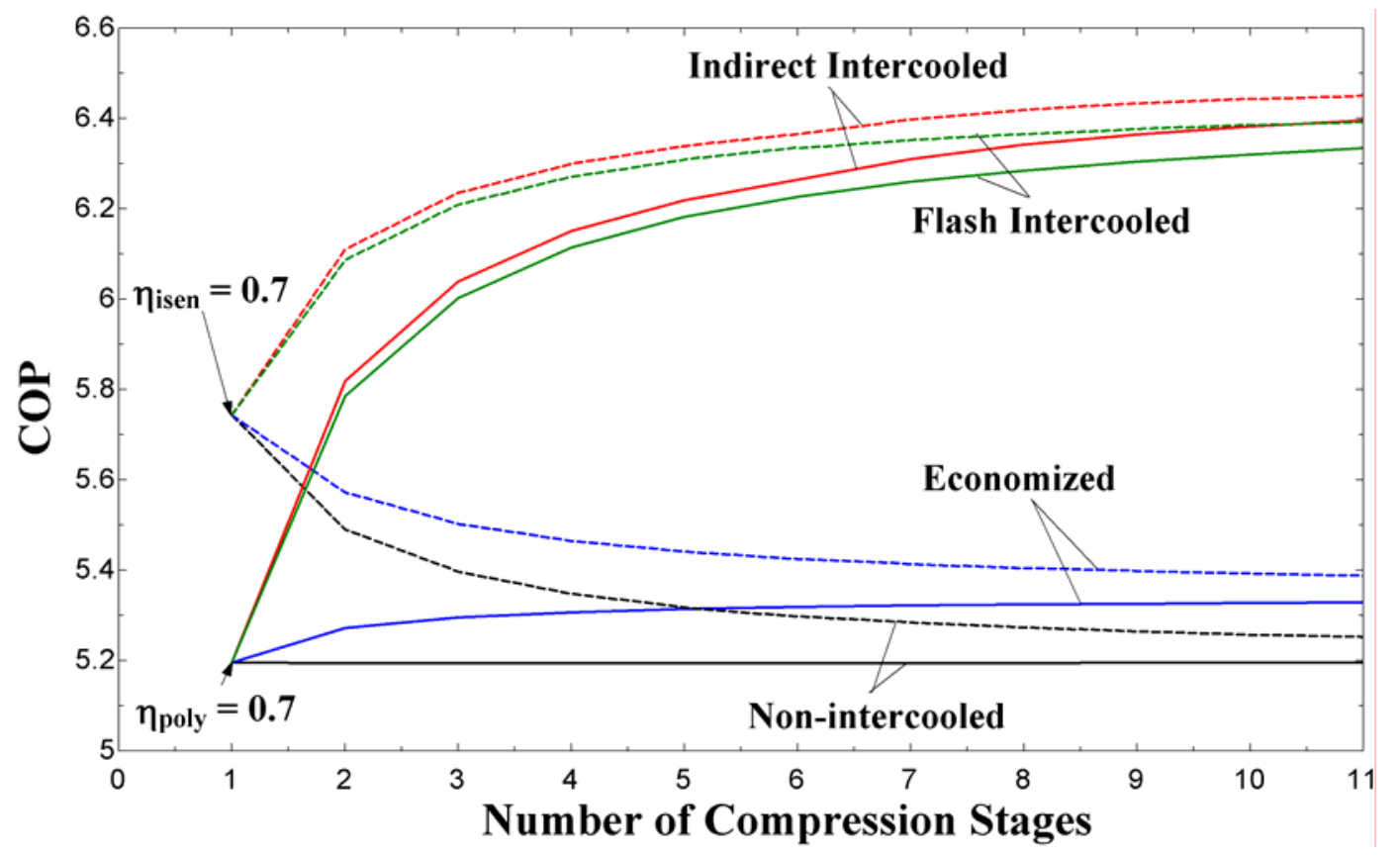

Figure 20 Cycle COP as a function of the number of stages of compression for a flash intercooled cycle using the "Condenser Water Bleed Off" configuration compared with indirect intercooled, economized, and non-intercooled.

When comparing water with an R-134a cycle, the merit of flash intercooling relative to economizing is reversed. This is due to the fact that the irreversibility in the throttling process for $\mathrm{R}-134 \mathrm{a}$ is significantly higher than that of water, creating more flash gas. In the economized cycle, this gas can affect a larger amount of intercooling while reducing what is a relatively high evaporator inlet quality. In the case of flash intercooling an R-134a cycle, the throttling losses are large and the mass added in upstream compressors penalizes the cycle. Figure 21 illustrates the different effects of flash intercooling and economizing for water and R-134a. 


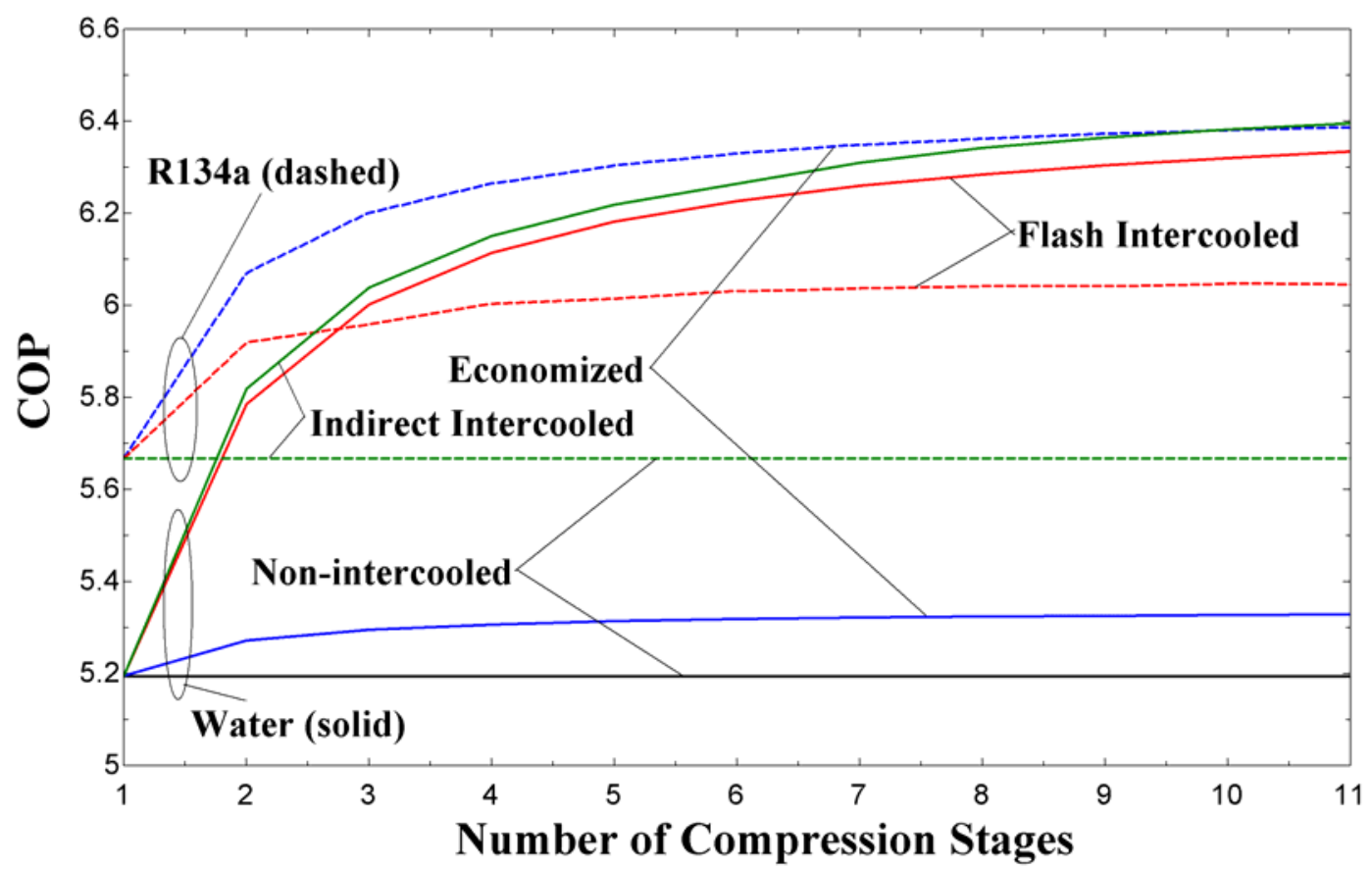

Figure 21 Cycle COP as a function of the number of compression stages for indirect intercooled, flash intercooled, economized, and non-intercooled cycles for both water and $\mathrm{R}-134 \mathrm{a}$ as a refrigerant.

Note that the flash and indirect-intercooled cycles significantly out-perform the other options for water as a refrigerant and the relative simplicity of the flash intercooled cycle over the indirect intercooled cycle makes it the best option. Indirect intercooling shows the best performance, but it is only slightly better than the flash intercooled cycle. The marginal benefit of the indirect intercooled cycle over the flash intercooled cycle is not likely to justify the added cost of an extra indirect-contact intercooler; a piece of equipment that is substantially more expensive than a direct-contact heat exchanger. For this reason, the indirect intercooled cycle was abandoned.

\subsubsection{Direct Intercooled and Liquid Subcooled Cycles}

Direct intercooled and liquid subcooled cycles rely on heat transfer between superheated vapor bubbles pumped through a liquid medium. The vapor leaving the low 
pressure compressor "bubbles up" through standing liquid refrigerant obtained from an upper stage expansion process. Upon reaching the surface of the liquid, the saturated vapor can be sent to the high stage compressor. The liquid subcooled cycle utilizes a modification to the direct intercooled cycle. An indirect heat exchanger is submerged in the direct-contact heat exchanger that is intercooling at an intermediate temperature and pressure. This serves to cool the liquid leaving the condenser to a temperature below its saturation temperature. Figures 22 and 23 are schematics of a 2-stage direct intercooled cycle and a 2-stage liquid subcooled cycle, respectively. Figure 24 is a T-s diagram of a 2-stage direct intercooled cycle.

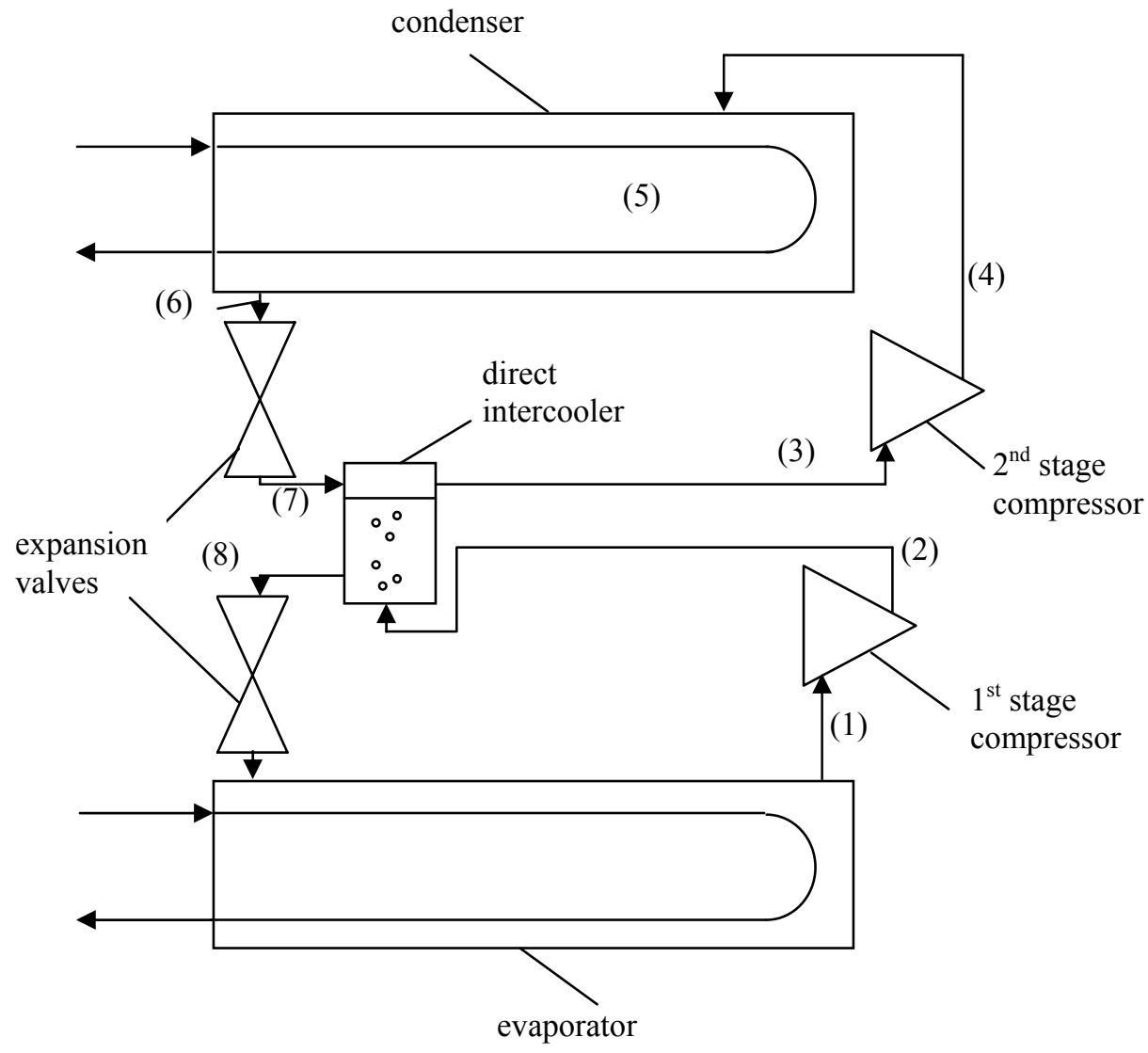

Figure 22 Schematic of a two-stage direct intercooled cycle. 


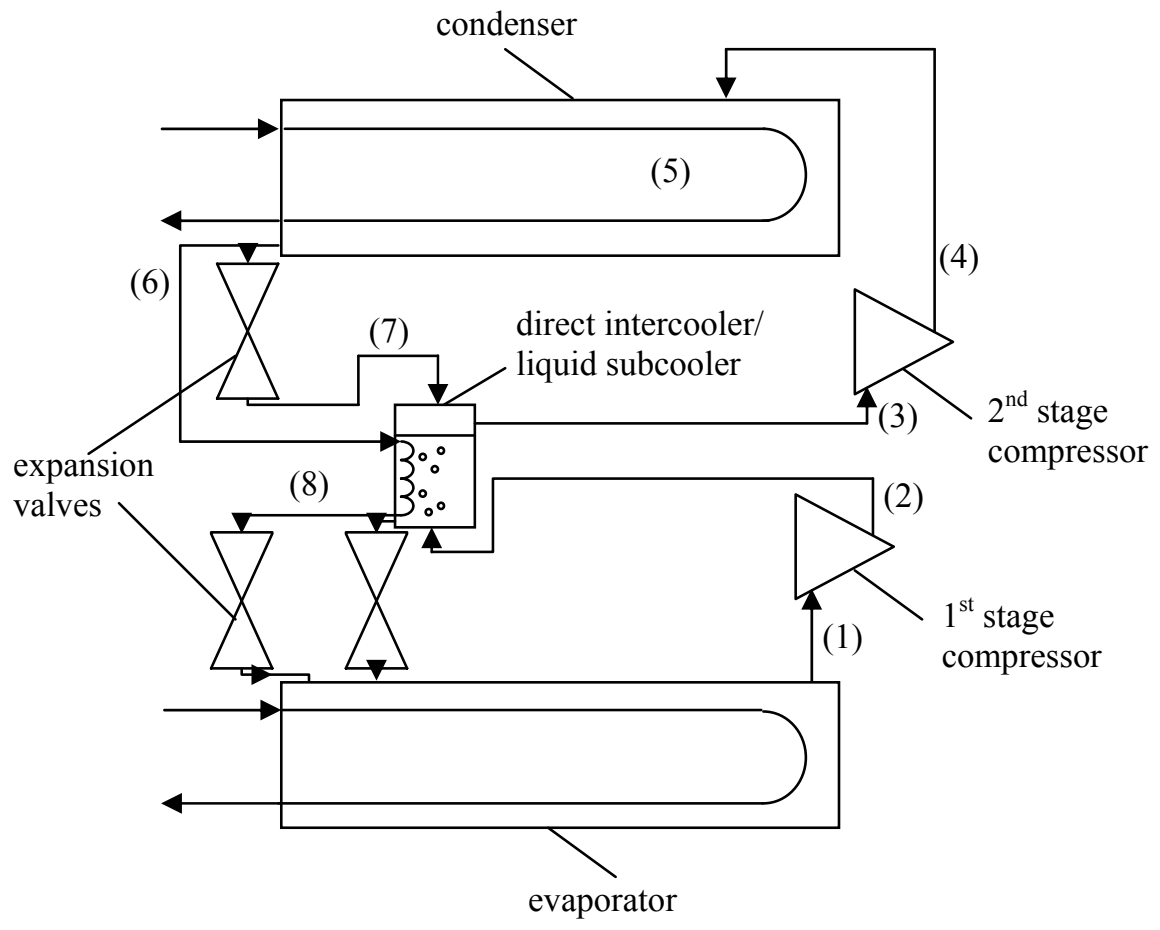

Figure 23 Schematic of a two-stage liquid subcooled cycle.

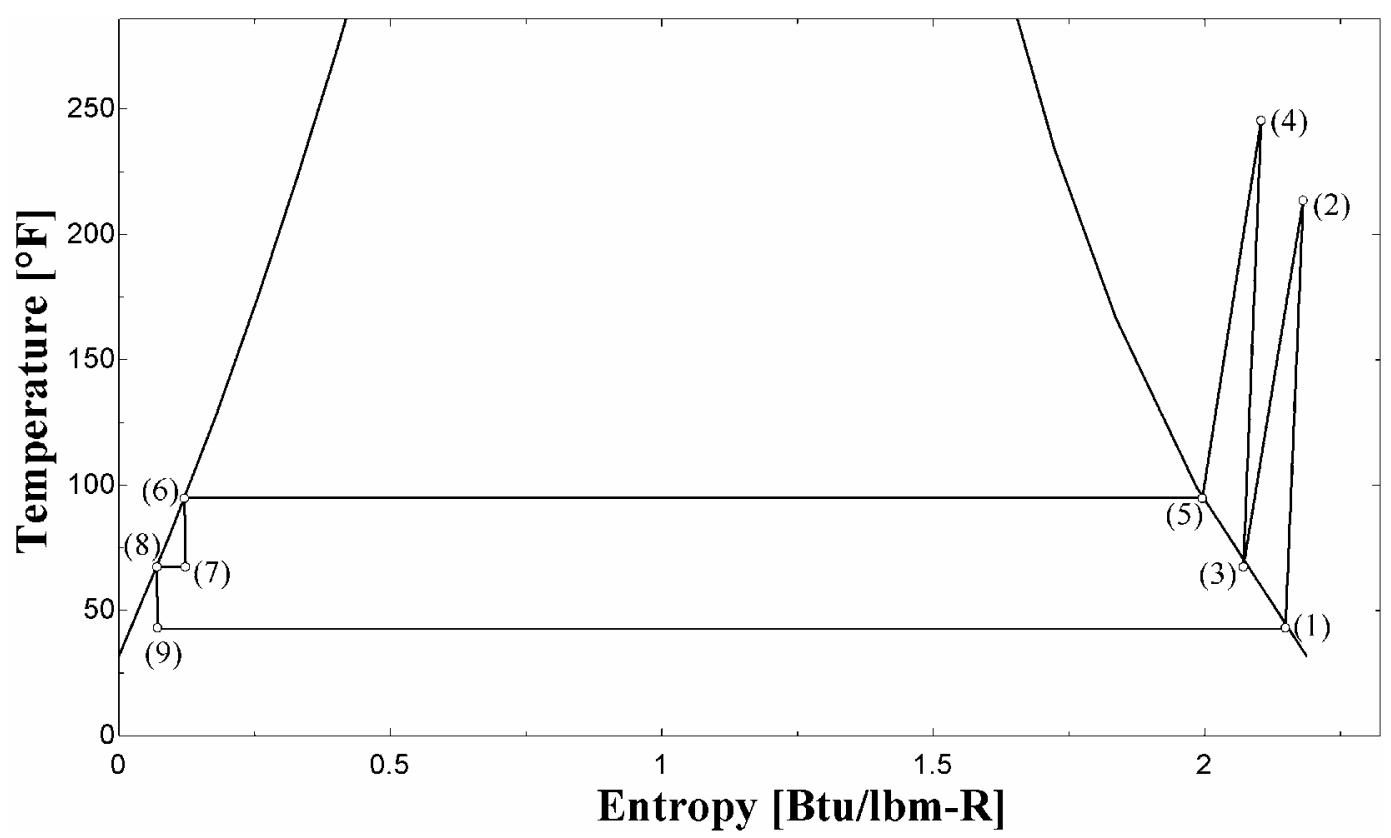

Figure 24 T-s diagram of a 2-stage direct intercooled cycle.

Direct intercooled and liquid sub-cooled cycle configurations were rejected for this application due to the unique characteristics of water as a refrigerant. These cycles 
both require some liquid depth of refrigerant (e.g. for the direct intercooled cycle, state (2) will be at a higher pressure than state (3)). The very low pressures and low pressure differences within the water refrigeration cycle together with the relatively high density of liquid water make it very sensitive to any hydrostatic pressure gradients within the cycle. Figure 25 illustrates the effect of a small hydrostatic pressure rise (associated with a small amount of standing water) on the COP of the direct and liquid subcooled refrigeration cycle (assuming a 2-stage system with perfect compressors and heat exchangers).

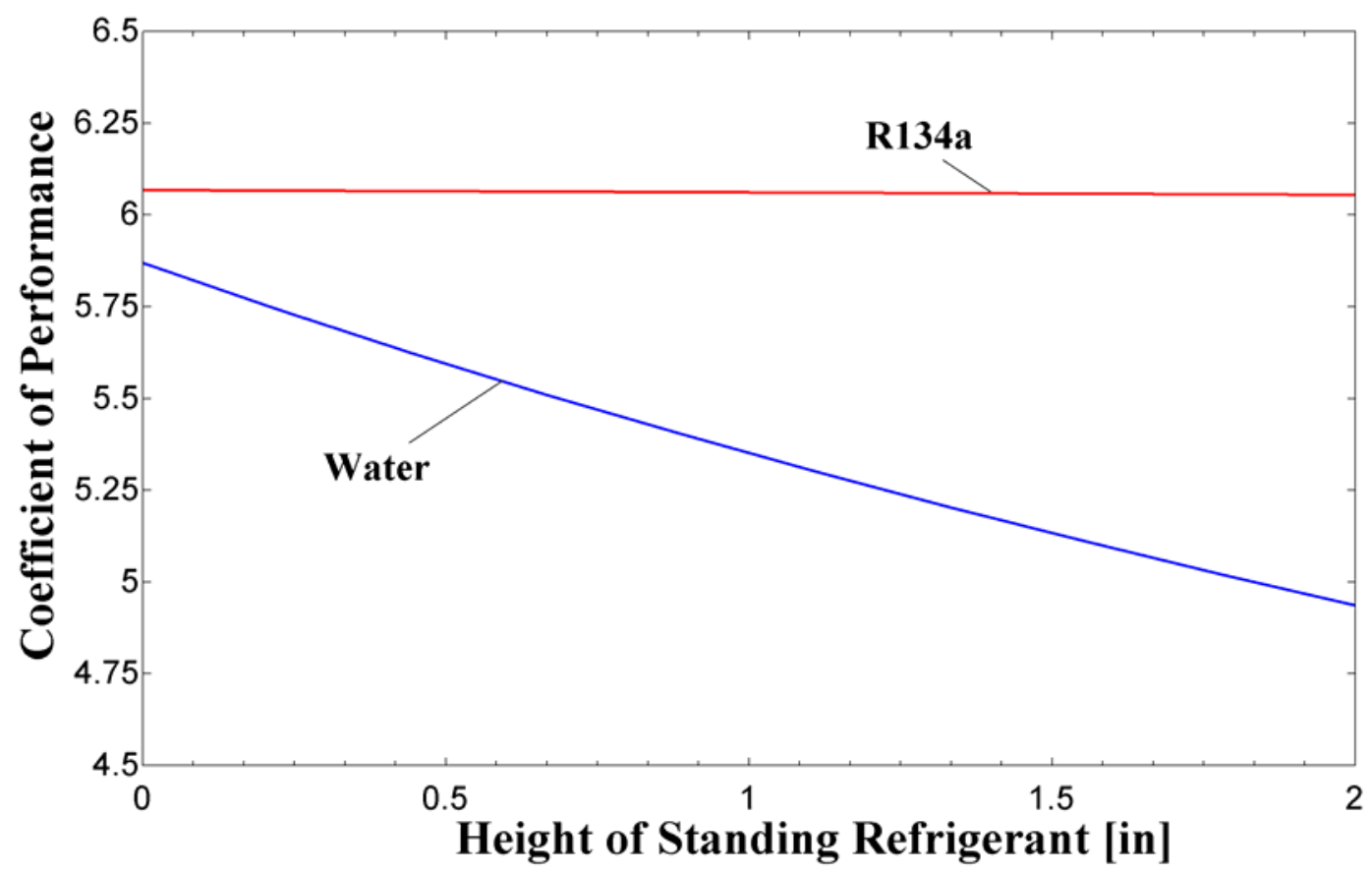

Figure $25 \mathrm{COP}$ as a function of the height of any standing refrigerant in the cycle for a 2stage direct intercooled cycle for water and R-134a as refrigerants.

Note that the small pressure differences and large density of liquid water make the water cycle extremely sensitive to hydrostatic head which precludes the use of cycle configurations that require standing water. The R-134a cycle is very insensitive to the hydrostatic head because the overall pressure lift is so much larger. 


\subsection{Modeling Results}

This section serves as a check for the accuracy of the thermodynamic assumptions and calculations performed for each cycle configuration before moving on to the component models. It also wraps up the conclusions drawn from the cycle modeling.

\subsubsection{Model Verification}

The cycle models presented in Section 3.1 were manipulated so that the assumptions used are nearly the same as those described by van Orshoven et al. (1991). In this limit, the results of the models developed here compare favorably with the published results. The ratio of the cycle COP to the COP of a reversible cycle operating between the condenser temperature and the evaporator temperature $\left(\mathrm{COP} / \mathrm{COP} \mathrm{Carnot}_{\mathrm{t}}\right)$ is shown as a function of the condenser water exit temperature. This COP ratio is compared with the single- and multi-stage (7-stage) non-intercooled, economized, flash intercooled, and indirect intercooled cycles. The $\mathrm{COP}_{\text {Carnot }}$ is calculated using the following equation.

$$
\mathrm{COP}_{\text {Carnot }}=\frac{1}{\left(\frac{\mathrm{T}_{\text {condenser }}}{\mathrm{T}_{\text {evaporator }}}-1\right)}
$$

where all temperatures are on the absolute scale.

Figure 26 shows a comparison of our calculations with van Orshoven et al. (1991). There are slight differences between the results, most notably between the performance of the indirect intercooled and the flash intercooled. It is not clear where these small discrepancies come from but it is possible that the earlier report used different database for water properties or slightly different assumptions for the component performance. 


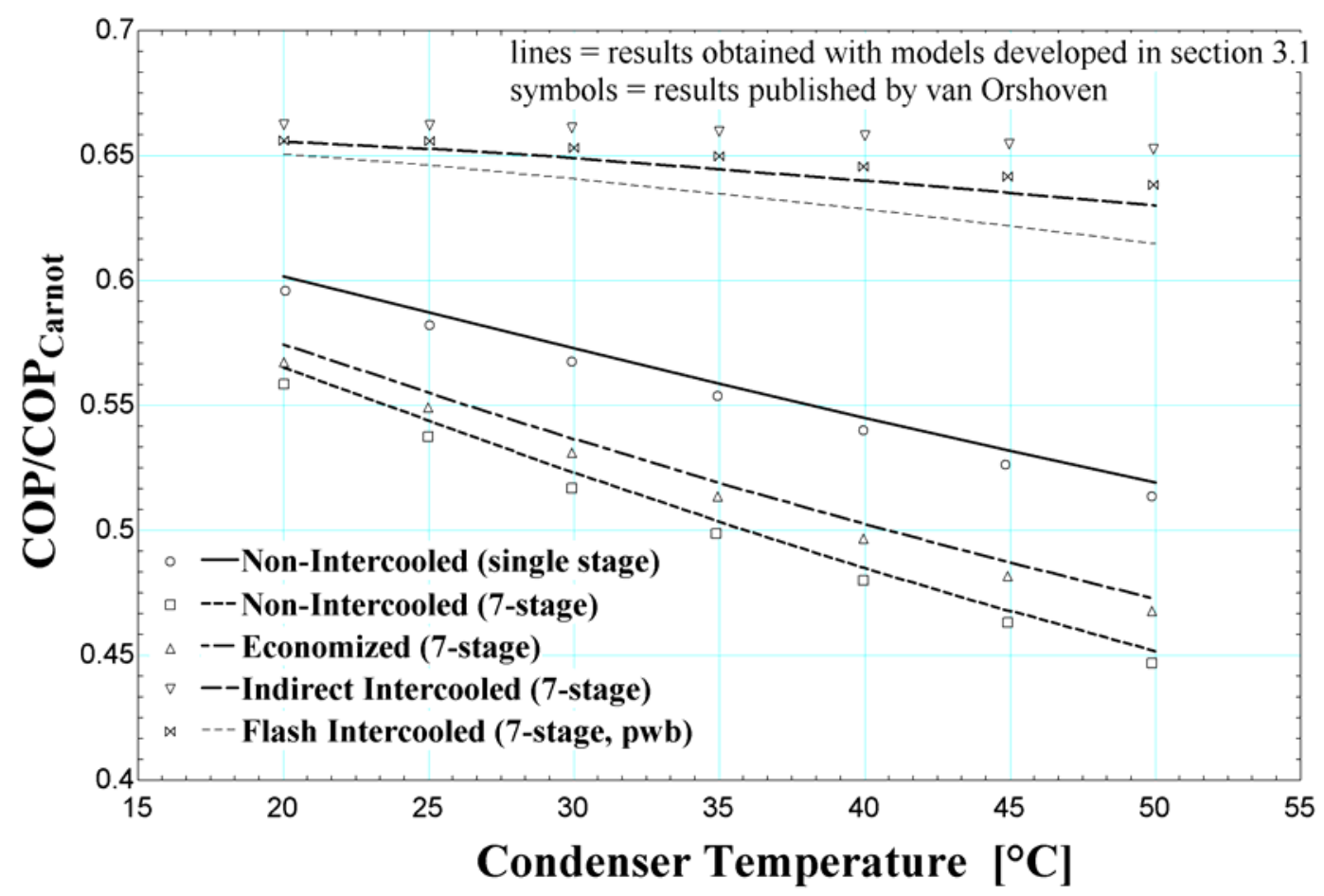

Figure $26 \mathrm{COP} / \mathrm{COP}_{\text {Carnot }}$ based on the models developed here and the results described by van Orshoven et al. (1991) for an array of cycle configurations.

\subsubsection{Cycle Model Results}

Figure 20 illustrates the predicted COP as a function of the number of stages for the non-intercooled cycle, the economized cycle, the flash intercooled cycle, and the indirect intercooled cycle. Figure 20 was prepared for the baseline conditions specified by ARTI using a consistent set of assumed component-level performance parameters $\left(\Delta \mathrm{T}_{\text {evap }}=0.5^{\circ} \mathrm{C}, \Delta \mathrm{T}_{\text {cond }}=0^{\circ} \mathrm{C}, \eta_{\text {comp }}=70 \%\right)$. These component performance parameters can be changed to reflect different types of hardware (e.g. indirect vs. direct condensers and evaporators) and the distribution of the stage pressure ratios can be varied (e.g. equal pressure ratio vs. equal isentropic head) but the general conclusions that are drawn from these cycle analyses will not change. These conclusions are as follows:

o The non-intercooled water cycle has a relatively low coefficient of performance. The primary loss mechanism in the cycle is related to the large 
amount of superheat associated with the compression process and there is very little throttling loss. Therefore any beneficial cycle configuration will provide a large amount of intercooling in a multi-stage compression process.

o Economizing provides only a small amount of intercooling leading to a marginal improvement in the performance of the non-intercooled cycle. The small amount of intercooling is related to the small amount of entropy produced in the expansion processes.

o Economizing for an R-134a cycle is the most attractive configuration for this refrigerant from a thermodynamic basis. The larger throttling losses (compared with water) complement the lower superheat loss well.

o Flash intercooling in water as refrigerant cycles is an effective method of improving cycle performance as it provides a relatively large amount of intercooling using only direct-contact heat exchangers, components that should have a small capital cost penalty.

o Flash intercooling does not suit an R-134a cycle because the throttling loss is not addressed with flash intercooling and superheating is a minor loss in this cycle.

o Indirect intercooling is slightly better than flash intercooling because the upper stage compressors do not have to carry the extra mass flow rate associated with the flash vapor; however, indirect intercooling requires relatively expensive indirect heat exchangers that are not likely to be justified by the marginal improvement over flash intercooling. 
o Indirect intercooling does not work in an R-134a cycle using the temperatures specified by ARTI. There is not enough superheat in the low pressure compressor to reach even the condenser water temperature. Thus, desuperheating is not possible and the cycle acts like a non-intercooled cycle.

o Direct intercooling, as shown in the cycle schematic in Figure 22, is not feasible for water as a refrigerant. The low operating pressures of a water cycle precludes any standing water in a direct-contact heat exchanger. Figure 25 illustrates the penalty of any height of standing water.

o The liquid subcooled cycle is not feasible for the same reason that the direct intercooled cycle is unattractive; it uses standing water. In addition, it has the added expense of another heat exchanger. For this reason, this cycle was never modeled.

o Flash intercooling is the cycle of choice for water as a refrigerant because it provides significant intercooling in a practical configuration using only directcontact heat exchangers.

Based on the discussion above it is possible to select the flash intercooled cycle as the most appropriate cycle configuration. The 2-stage flash intercooled cycle is referred to as the baseline cycle and the thermodynamic model used to predict the performance of the baseline cycle is described in detail in the following section. The component level models described in Chapter 4 are implemented as sub-modules in the framework of the baseline cycle model described here. 


\subsection{Baseline Cycle}

The baseline cycle selected for this project is a 2-stage flash intercooled cycle. This section describes the specific calculations required to predict the performance of the baseline cycle. The condenser water and chilled water temperatures and the required cooling load are known from the ARTI-specified conditions. The temperatures allow the calculation of the nominal high and low pressures in the cycle based on the properties of the refrigerant and the approach temperature differences in each heat exchanger. The pressures at the other state points can be determined based on the number of stages and the algorithm selected to divide the pressure ratio among the stages.

$$
\begin{aligned}
& \mathrm{p}_{\text {condenser }}=\operatorname{pressure}\left(\text { Steam }, \mathrm{T}=\mathrm{T}_{\text {condenser }}, \mathrm{x}=0\right) \\
& \mathrm{p}_{\text {evaporator }}=\operatorname{pressure}\left(\text { Steam }, \mathrm{T}=\mathrm{T}_{\text {evaporator }}, \mathrm{x}=0\right)
\end{aligned}
$$

$\mathrm{T}_{\text {condenser }}$ and $\mathrm{T}_{\text {evaporator }}$ are determined from:

$$
\begin{aligned}
& \mathrm{T}_{\text {condenser }}=\mathrm{T}_{\text {coolwater,out }}+\Delta \mathrm{T}_{\text {cond }} \\
& \mathrm{T}_{\text {evaporator }}=\mathrm{T}_{\text {chillwater,out }}-\Delta \mathrm{T}_{\text {evap }}
\end{aligned}
$$

where $\Delta \mathrm{T}_{\text {cond }}$ and $\Delta \mathrm{T}_{\text {evap }}$ are the condenser and evaporator approach temperatures, respectively. 
It was found that the most computationally efficient model resulted when equal pressure ratios were used for each compression stage. The overall performance of the cycle was not heavily dependent on how the pressure ratio was divided and therefore the results from this point forward are based on applying equal pressure ratio across each compression stage. The variables in the EES model are indexed to indicate the various state points within the cycle. This convention adds versatility to the program in that the number of stages of compression (NSC) can be changed to an arbitrary integer value without requiring a new program. Table 4 summarizes the convention for the indexed variables. The T-s diagram for the baseline cycle is repeated in Figure 27 for reference.

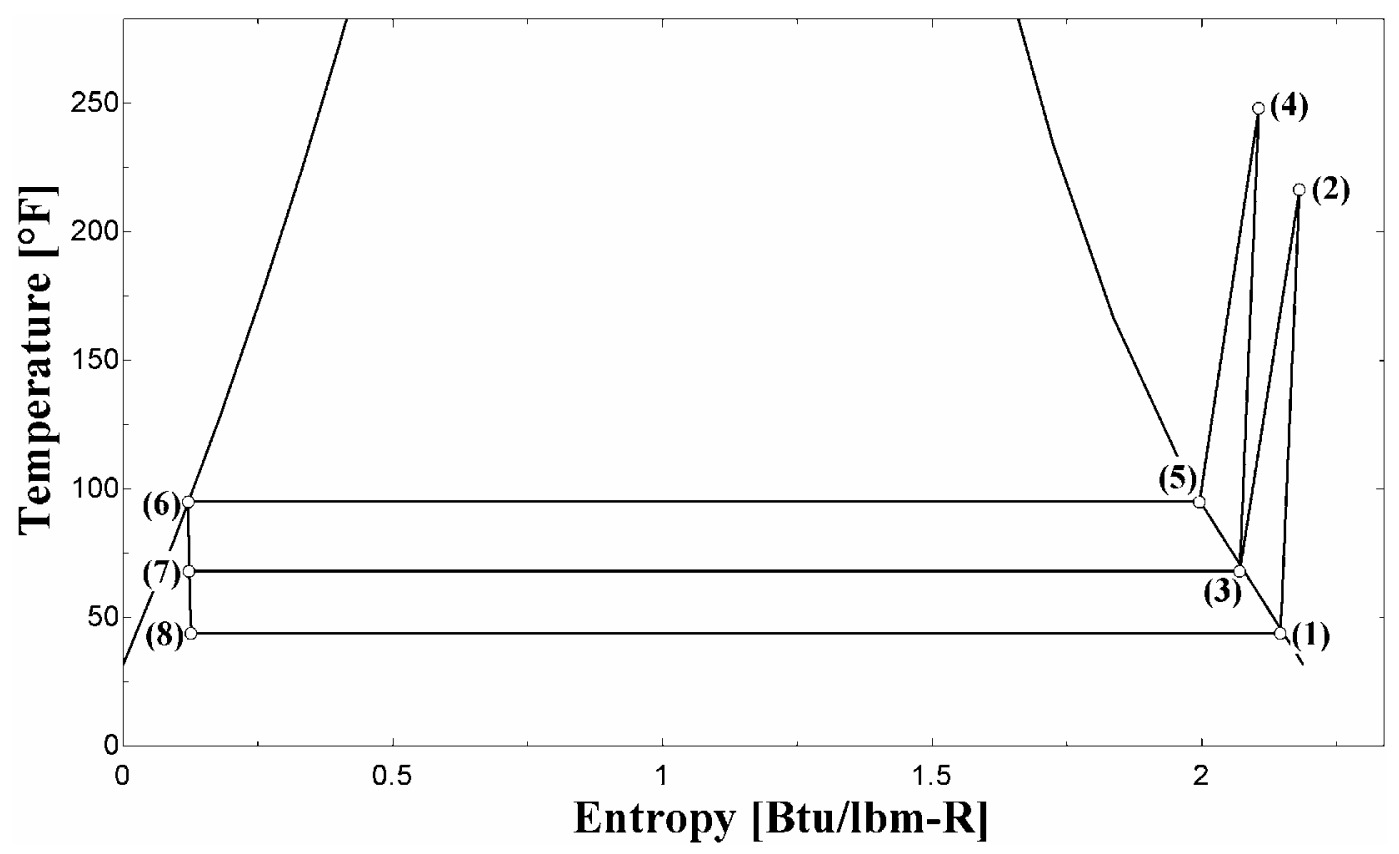

Figure 27 T-s diagram of the baseline cycle selected for this project: A 2-stage flash intercooled cycle. 
Table 4 Indexing convention for states within flash intercooled cycle.

\begin{tabular}{l|l|l} 
Location & Range & Index of State \\
\hline Compressor inlet & $\mathrm{j}=1 . . \mathrm{NSC}$ & $2 * \mathrm{j}-1$ \\
Compressor exit & $\mathrm{j}=1 . . \mathrm{NSC}$ & $2 * \mathrm{j}$ \\
Valve inlet & $\mathrm{j}=1 . . \mathrm{NSC}$ & $2 * \mathrm{NSC}+1+\mathrm{j}$ \\
Final valve exit & - & $3 * \mathrm{NSC}+2$ \\
On the vapor dome & - & $2 * \mathrm{NSC}+1$
\end{tabular}

The compressor suction pressures are calculated (assuming equal pressure ratios).

$$
p_{2 \cdot j-1}=p_{\text {evaporator }} \cdot\left(\frac{p_{\text {condenser }}}{p_{\text {evaporator }}}\right)^{\frac{j-1}{N S C}} \text { for } j=1 \text { to NSC }
$$

The compressor discharge pressures are calculated.

$$
p_{2 \cdot j}=p_{\text {evaporator }} \cdot\left(\frac{p_{\text {condenser }}}{p_{\text {evaporator }}}\right)^{\frac{j}{N S C}} \text { for } j=1 \text { to NSC }
$$

The final indexed pressures are the intermediate expansion valve pressures.

$$
\mathrm{p}_{2 \cdot \mathrm{NSC}+1+\mathrm{j}}=\mathrm{p}_{2 \cdot(\mathrm{NSC}+1-\mathrm{j})} \text { for } \mathrm{j}=1 \text { to NSC }
$$

Pressure losses are calculated for the heat exchangers using the heat exchanger component model as described in Chapter 4. These pressure losses are applied to the pressure calculated above. The final expansion valve exit pressure is set equal to the pressure at state (1) and, for clarity in reading the T-s diagram, a state is defined at the high pressure level with a quality of 1 (see Figure 17).

All of the properties associated with the valve inlet and exit stages can be computed using the valve pressures and enthalpies (all valves are assumed isenthalpic). 


$$
\begin{array}{r}
h_{2 \cdot \mathrm{NSC}+2+\mathrm{j}}=\mathrm{h}_{2 \cdot \mathrm{NSC}+1+\mathrm{j}} \text { for } \mathrm{j}=1 \text { to NSC } \\
\mathrm{x}_{2 \cdot \mathrm{NSC}+2+\mathrm{j}}=\text { quality(Steam, } \mathrm{p}=\mathrm{p}_{2 \cdot \mathrm{NSC}+2+\mathrm{j}}, \mathrm{h}=\mathrm{h}_{2 \cdot \mathrm{NSC}+2+\mathrm{j}} \text { ) for } \mathrm{j}=1 \text { to NSC } \\
\mathrm{s}_{2 \cdot \mathrm{NSC}+2+\mathrm{j}}=\operatorname{entropy}\left(\operatorname{Steam}, \mathrm{p}=\mathrm{p}_{2 \cdot \mathrm{NSC}+2+\mathrm{j}}, \mathrm{h}=\mathrm{h}_{2 \cdot \mathrm{NSC}+2+\mathrm{j}} \text { ) for } \mathrm{j}=1\right. \text { to NSC } \\
\mathrm{T}_{2 \cdot \mathrm{NSC}+2+\mathrm{j}}=\text { temperature(Steam, } \mathrm{p}=\mathrm{p}_{2 \cdot \mathrm{NSC}+2+\mathrm{j}}, \mathrm{h}=\mathrm{h}_{2 \cdot \mathrm{NSC}+2+\mathrm{j}} \text { ) for } \mathrm{j}=1 \text { to NSC } \\
\mathrm{v}_{2 \cdot \mathrm{NSC}+2+\mathrm{j}}=\text { volume}\left(S t e a m, p=p_{2 \cdot \mathrm{NSC}+2+\mathrm{j}}, \mathrm{h}=\mathrm{h}_{2 \cdot \mathrm{NSC}+2+\mathrm{j}} \text { ) for } \mathrm{j}=1\right. \text { to NSC }
\end{array}
$$

The properties associated with the compressor exit states can be determined using the compressor polytropic efficiency $\left(\eta_{\text {compressor }}\right)$. This polytropic efficiency is predicted by the compressor component level model described in Chapter 4.

$$
\begin{array}{r}
\left.\mathrm{h}_{\mathrm{is}, 2 \cdot \mathrm{j}}=\text { enthalpy(Steam, } \mathrm{p}=\mathrm{p}_{2 \cdot \mathrm{j}}, \mathrm{s}=\mathrm{s}_{2 \cdot \mathrm{j}-1}\right) \text { for } \mathrm{j}=1 \text { to NSC } \\
\mathrm{h}_{2 \cdot \mathrm{j}}=\mathrm{h}_{2 \cdot \mathrm{j}-1}+\frac{\mathrm{h}_{\text {is }, 2 \cdot \mathrm{j}}-\mathrm{h}_{2 \cdot \mathrm{j}-1}}{\eta_{\text {compressor }}} \text { for } \mathrm{j}=1 \text { to NSC } \\
\mathrm{T}_{2 \cdot \mathrm{j}}=\text { temperature }\left(\mathrm{p}=\mathrm{p}_{2 \cdot \mathrm{j}}, \mathrm{h}=\mathrm{h}_{2 \cdot \mathrm{j}}\right) \text { for } \mathrm{j}=1 \text { to NSC } \\
\left.\mathrm{s}_{2 \cdot \mathrm{j}}=\text { entropy(Steam, } \mathrm{p}=\mathrm{p}_{2 \cdot \mathrm{j}}, \mathrm{h}=\mathrm{h}_{2 \cdot \mathrm{j}}\right) \text { for } \mathrm{j}=1 \text { to NSC } \\
\left.\mathrm{v}_{2 \cdot \mathrm{j}}=\text { volume(Steam, } \mathrm{p}=\mathrm{p}_{2 \cdot \mathrm{j}}, \mathrm{h}=\mathrm{h}_{2 \cdot \mathrm{j}}\right) \text { for } \mathrm{j}=1 \text { to NSC }
\end{array}
$$

The mass flow rate through each subsequent compressor increases due to the mass added in each of the flash intercooling processes. The mass flow rate in each compressor can be computed based on an energy balance on the intercoolers:

$$
\begin{array}{r}
\dot{\mathrm{m}}_{\text {flash }, 2 \cdot \mathrm{NSC}+1+\mathrm{j}}=\dot{\mathrm{m}}_{\mathrm{NSC}+1-\mathrm{j}} \cdot\left(\frac{\mathrm{h}_{2 \cdot \mathrm{NSC}-2 \cdot \mathrm{j}+3}-\mathrm{h}_{2 \cdot \mathrm{NSC}-2 \cdot \mathrm{j}+2}}{\mathrm{~h}_{2 \cdot \mathrm{NSC}+\mathrm{j}+1}-\mathrm{h}_{2 \cdot \mathrm{NSC}-2 \cdot \mathrm{j}+3}}\right) \text { for } \mathrm{j}=2 \text { to NSC } \\
\dot{\mathrm{m}}_{\mathrm{j}}=\dot{\mathrm{m}}_{\mathrm{j}-1}+\dot{\mathrm{m}}_{\text {flash }, 3 \cdot \mathrm{NSC}-\mathrm{j}+3} \text { for } \mathrm{j}=2 \text { to NSC }
\end{array}
$$

Note that the amount of liquid needed for the refrigerant injection is calculated by assuming that the compressor inlet states are maintained at the saturated vapor line plus some temperature difference related to the performance of the intercoolers. The COP can be computed using the required compressor power, $\dot{\mathrm{W}}_{\text {comp }}$, and the specified cooling load.

$$
\dot{\mathrm{q}}_{\text {evap }}=1000 \text { tons }\left(1.2\left(10^{7}\right) \mathrm{Btu} / \mathrm{h}\right)
$$




$$
\begin{gathered}
\dot{\mathrm{W}}_{\text {comp }}=\sum_{\mathrm{j}=1}^{\mathrm{NSC}} \dot{\mathrm{m}}_{\mathrm{NSC}+1-\mathrm{j}} \cdot\left(\mathrm{h}_{2 \cdot \mathrm{j}}-\mathrm{h}_{2 \cdot \mathrm{j}-1}\right) \\
\mathrm{COP}=\frac{\dot{\mathrm{q}}_{\text {evap }}}{\dot{\mathrm{W}}_{\text {net }}}
\end{gathered}
$$

where $\dot{\mathrm{W}}_{\text {net }}$ includes the compressor power and additional power to the run the pumps, as predicted by the pump component models described in Chapter 4.

The flash intercooled cycle model described here provides the framework for the detailed system model, allowing the component models to be separately integrated. Using this flash intercooled model, it is possible to estimate the effect of varying the heat exchanger approach temperatures on the cycle performance. Figures 28 and 29 show how the COP varies with the condenser and evaporator approach temperature differences, respectively, for the baseline water cycle as well as a single stage R-134a cycle. The model is also able to provide some indication of the differences between a water cycle and an R-134a cycle in terms of how a heat exchanger pressure drop affects the cycle performance. Figure 30 shows how the COP is affected by pressure drop for a 2-stage flash intercooled water cycle and for a single stage R-134a cycle. 


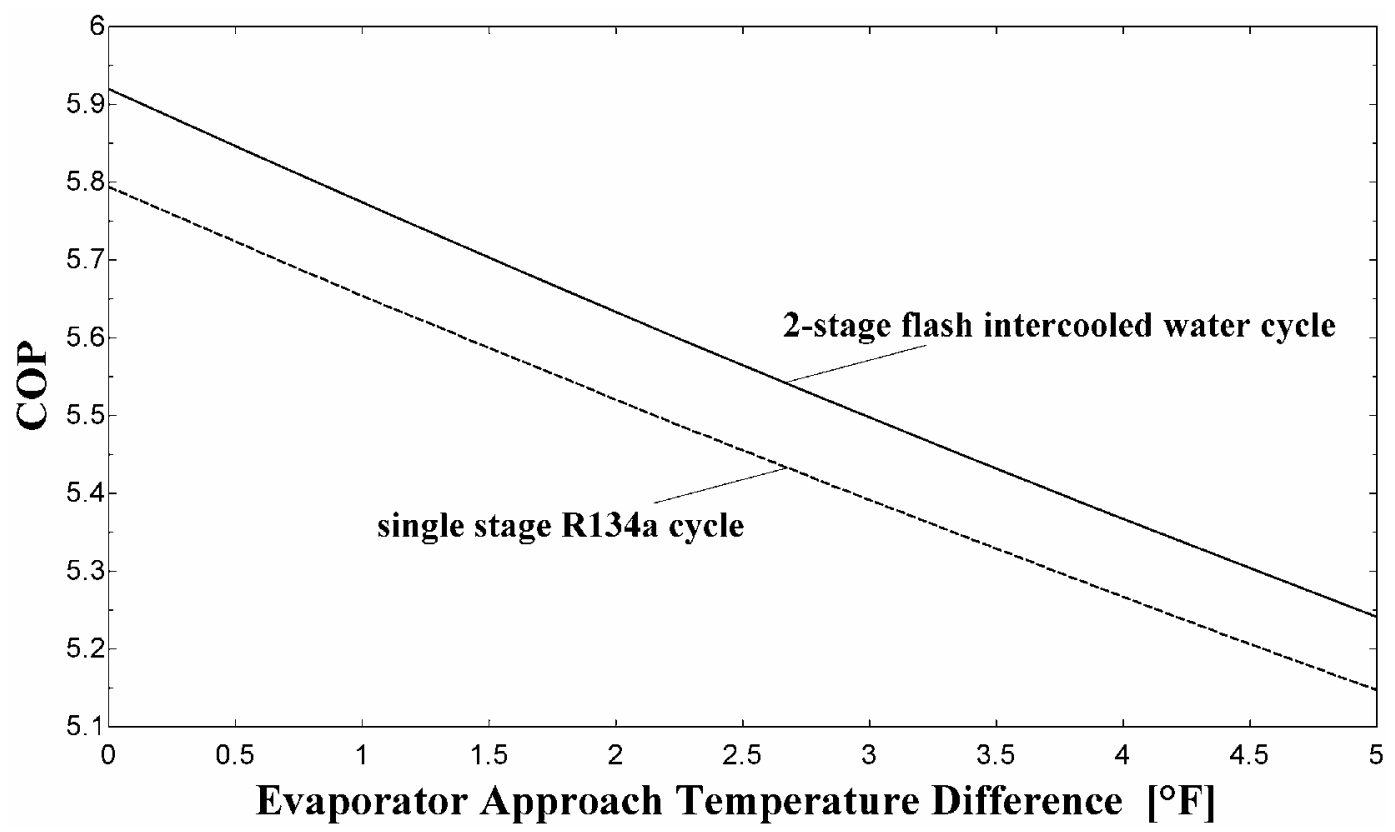

Figure 28 COP vs. evaporator approach temperature difference for a water 2-stage flash intercooled cycle and a single stage R-134a cycle.

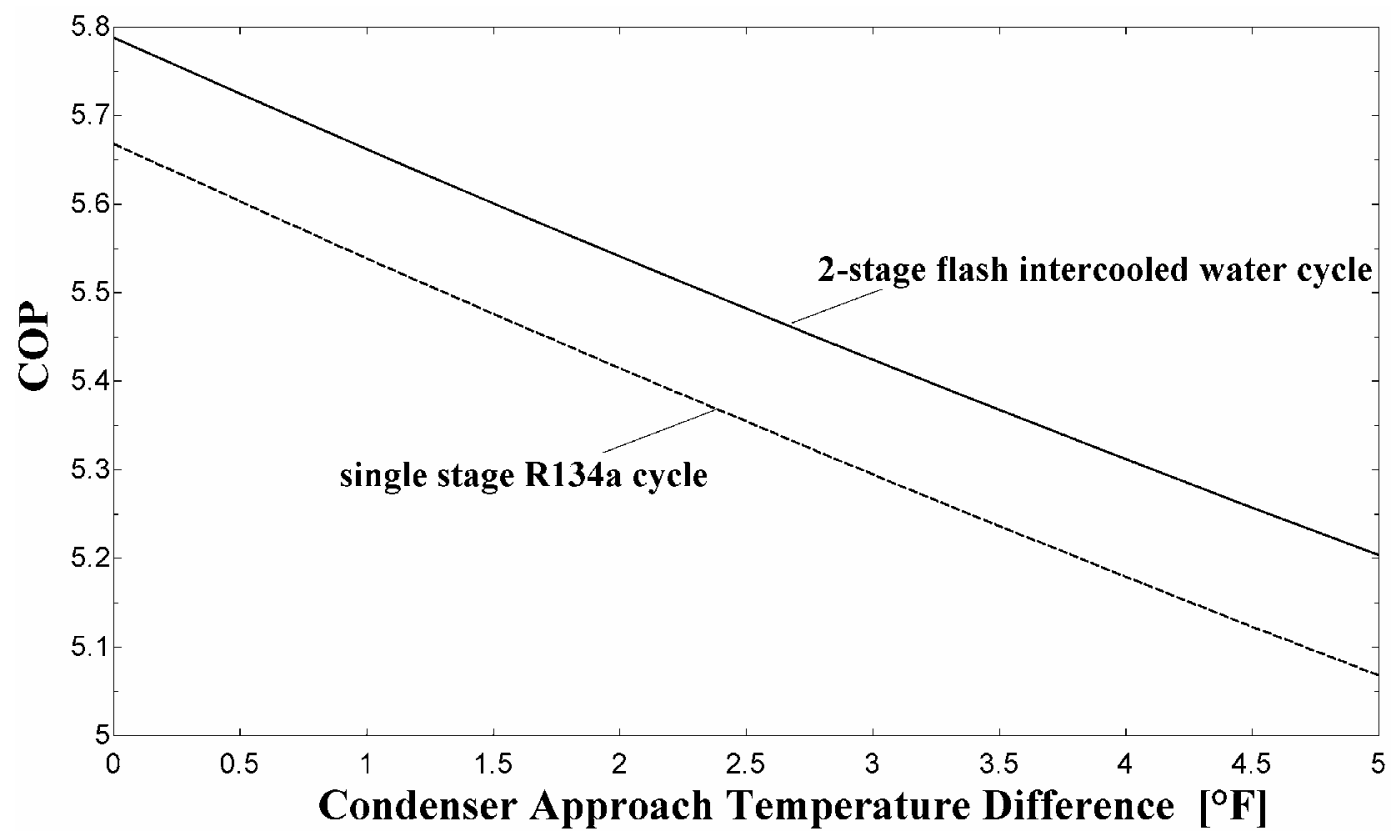

Figure 29 COP vs. condenser approach temperature difference for a water 2-stage flash intercooled cycle and a single stage R-134a cycle. 


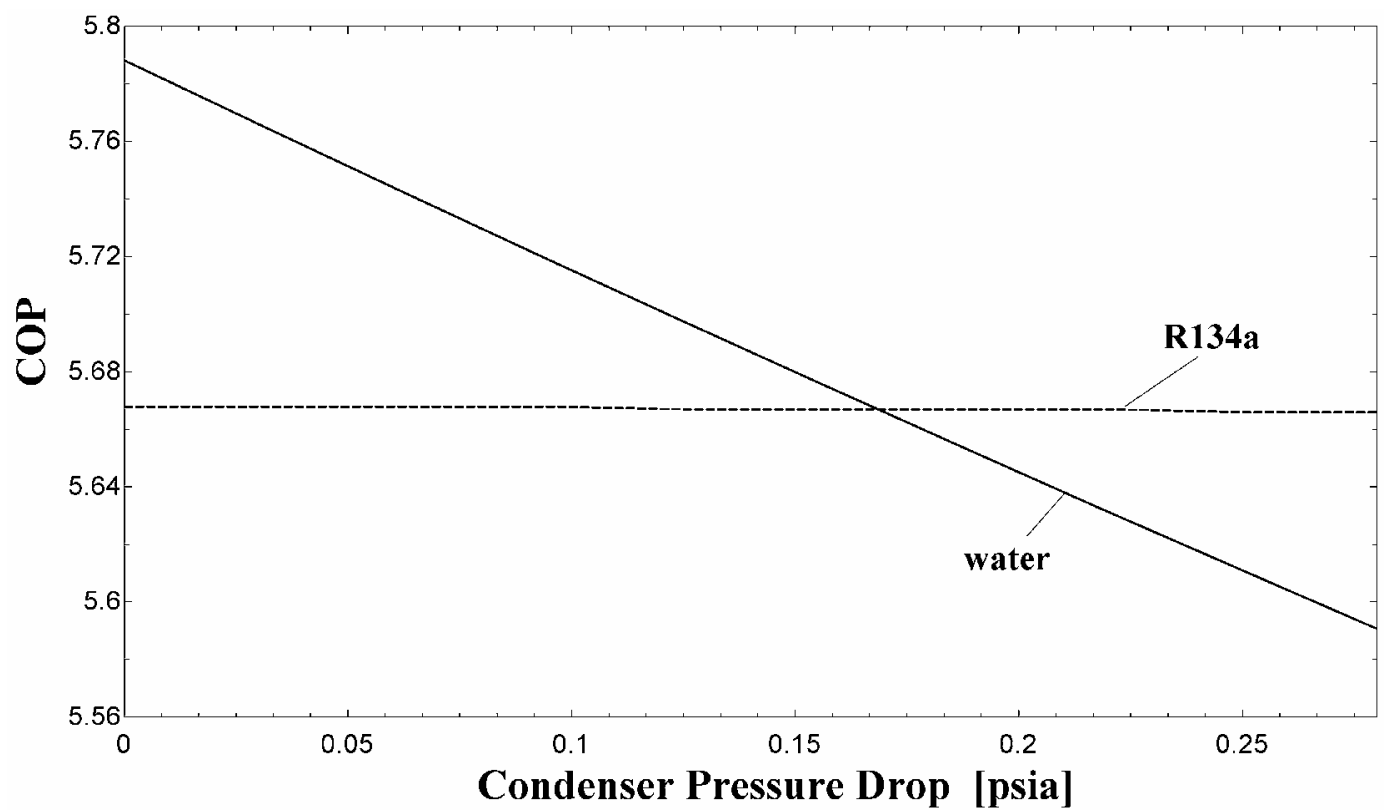

Figure 30 COP vs. condenser pressure drop for a water 2-stage flash intercooled cycle and a single stage R-134a cycle. 


\section{CHAPTER 4 SYSTEM MODEL}

This chapter presents a description of the detailed component models developed to create the full system models of the refrigeration cycles analyzed and compared in Chapter 5. The baseline system model for R-134a as a refrigerant is a single-stage centrifugal compressor, using a falling film condenser, and flooded evaporator. This cycle provides a useful point of comparison for the water-based cycles in the economic analysis. The most attractive water cycle configuration identified in Chapter 3 is the flash-intercooled, two-stage cycle using a permutation of direct and indirect-contact heat exchangers. The first section of this chapter describes the models for each of the components used in the conventional, R-134a system model and the second section describes the models for each of the components that are required for the water system models.

\subsection{Conventional, R-134a System Model}

This section is divided into four parts corresponding to detailed descriptions of the key components of a conventional single-stage R-134a cycle. In Section 4.1.1, the model of a single-stage centrifugal compressor model is described. In Section 4.1.2, the falling film indirect-contact, R-134a-to-water condenser model is presented. Section 4.1.3 describes the indirect-contact, R-134a-to-water flooded evaporator model. Section 4.1.4 describes the models of the chilled water and condenser water pumps.

\subsubsection{Compressor Model}

The operating conditions characterizing large chillers lead to the use of centrifugal compressors in industry today. Other compressor designs are not able to meet 
the efficiency targets and consequently they are not economically competitive. The compressor model described in this section is a single-stage centrifugal compressor.

\subsubsection{Performance}

The centrifugal compressor model is developed using the Engineering Equation Solver (EES) software so that it can easily be integrated with a single-stage R-134a refrigeration cycle model. The compressor model is used to estimate the performance, size, and cost of an R-134a compressor. The model builds upon simple relations for the performance of the sub-components that make up the centrifugal compressor, the nozzle, impeller, vaneless space, vaned diffuser, and the collector, in order to predict the static and stagnation states that the refrigerant experiences along a mean-line path between the compressor inlet and the exit. Table 5 shows the various parameters that are used in the model to capture the characteristics of the centrifugal compressor.

Table 5 Centrifugal compressor parameters.

\begin{tabular}{l|l|l} 
Parameter & Symbol & Default value* \\
\hline Slip factor & $\sigma$ & 0.9 \\
Nozzle efficiency & $\eta_{\text {nozzle }}$ & 0.95 \\
Hub-to-tip ratio & $\mathrm{r}_{\text {hub-to-tip }}$ & 0.2 \\
Impeller blade diffusion & Diffusion & 0.8 \\
Impeller blade total-to-total efficiency & $\eta_{\mathrm{TT} \text {,imp }}$ & 0.9 \\
\hline Vaneless space efficiency & $\eta_{\mathrm{vs}}$ & 0.95 \\
Mach number at vaneless space exit & $\mathrm{Ma}_{4}$ & 0.8 \\
Diffuser coefficient of pressure recovery & $\mathrm{C}_{\mathrm{pr}}$ & 0.7 \\
Diffuser inlet-to-exit velocity ratio & $\mathrm{c}_{5} / \mathrm{c}_{4}$ & 0.25 \\
Specific Speed & $\mathrm{n}_{\mathrm{s}}$ & 0.58 \\
\hline \multicolumn{2}{|l}{ * Value used in calculations in 4.1 .1} & \multicolumn{2}{|l}{}
\end{tabular}

Figure 31 is a schematic of a centrifugal compressor. The compressor model is broken into five distinct components and therefore six state points are calculated 
corresponding to the inlets to the nozzle, impeller blade, vaneless space, vaned diffuser, volute, and the compressor exit.

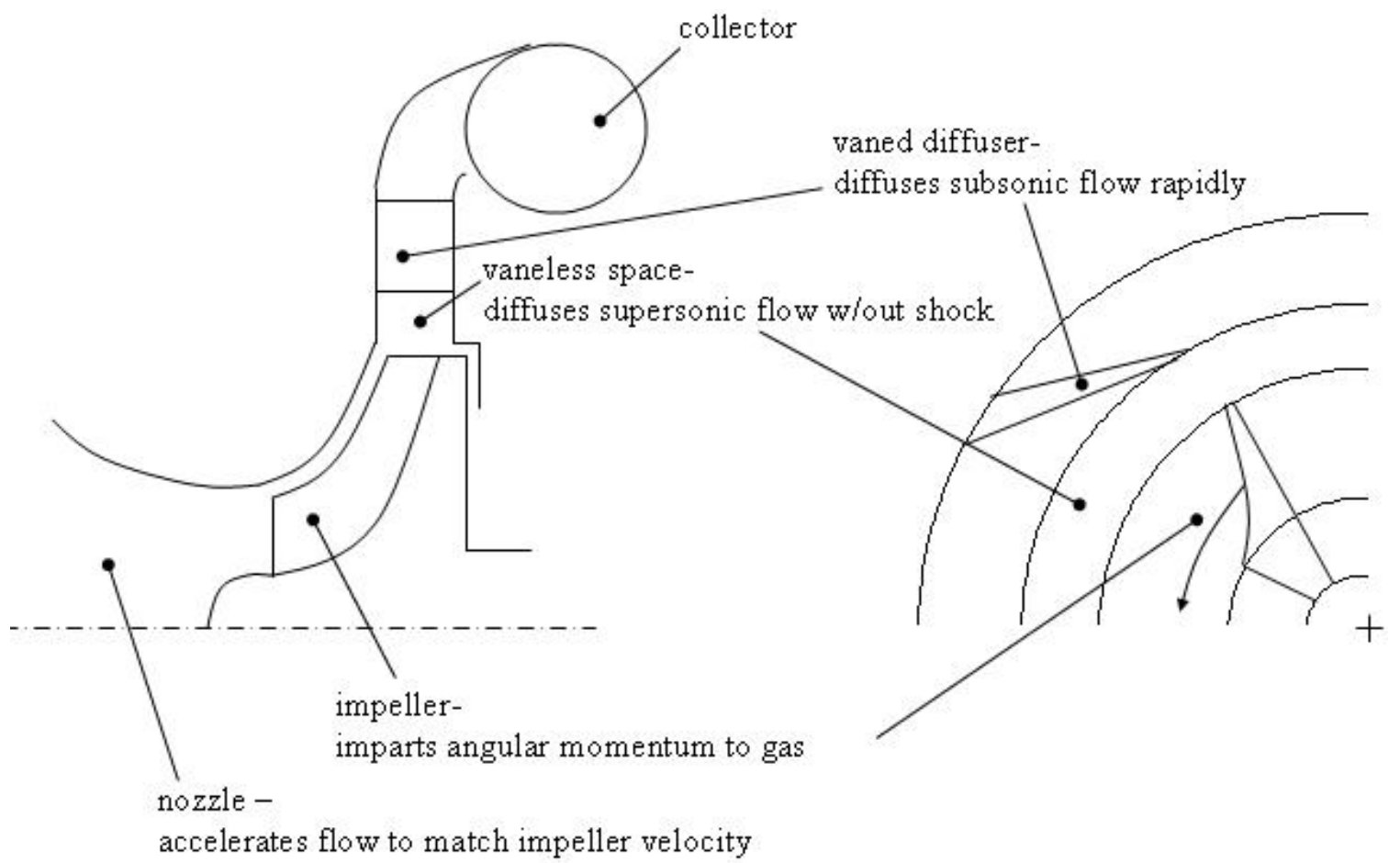

Figure 31 Schematic illustrating the components of a centrifugal compressor.

The state points are illustrated graphically in Figure 32 on a T-s diagram; both the static and stagnation state points are shown.
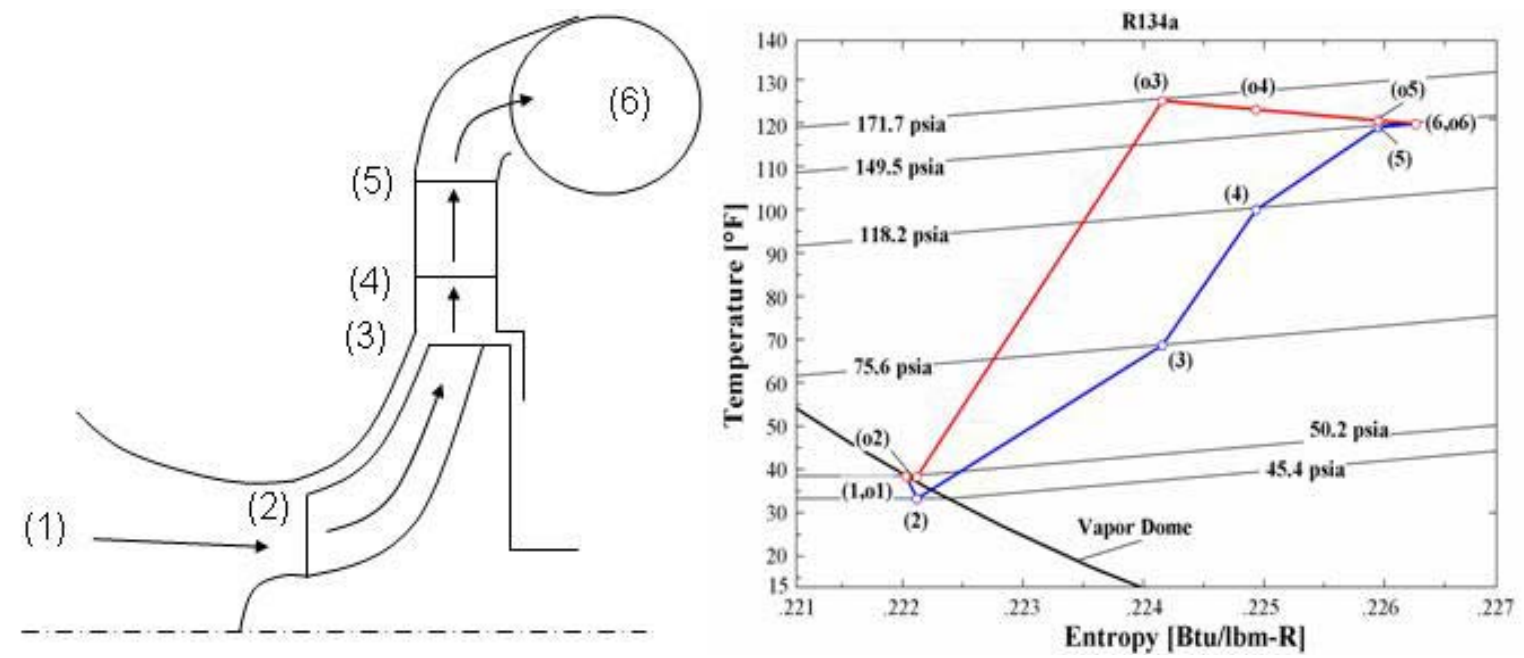

Figure 32 Schematic illustrating the state points within a compressor alongside the corresponding T-s diagram (R-134a cycle). 
There are a few notable features illustrated in Figure 32. If a design requirement is the prevention of condensation within the nozzle then some amount of superheat must be present at the compressor inlet. Figure 32 illustrates the drop in static temperature through the nozzle that is caused by the increase in velocity; this process presents the possibility that the refrigerant will enter the vapor dome, as illustrated in the figure. For $\mathrm{R}-134 \mathrm{a}$ the amount of superheat required to prevent condensation is relatively small and therefore does not impact the cycle significantly.

Notice that the entire change in the stagnation enthalpy occurs as it passes through the impeller blade and that states (o3), (o4), and (o5) lie on a line of constant enthalpy. This is because the impeller blade is the only component that imparts a work transfer to the refrigerant. All of the other components, excluding the nozzle, are in place in order to convert kinetic energy into static pressure. The purpose of the nozzle is to match the refrigerant velocity to the impeller blade velocity and thereby avoid incidence losses.

The first parameter that the compressor model calculates is the overall compressor pressure ratio, the ratio of the pressure at the condenser inlet to the evaporator exit.

$$
\mathrm{r}=\frac{\mathrm{p}_{\text {condenser,in }}}{\mathrm{p}_{\text {evaporator,out }}}
$$

In order to determine the initial centrifugal compressor state, the nozzle inlet, it is necessary to find the evaporator exit conditions. The chilled water return temperature is an ARTI-specified condition. The approach temperature difference of the evaporator and the condenser are specified in the model and will vary with the evaporator and condenser sizes. Details of the evaporator and condenser sub-programs are explained in subsequent sections. Thus, the evaporator exit temperature is equal to the chilled water return 
temperature minus the evaporator approach temperature. The evaporator pressure is calculated based on the assumption of saturated vapor refrigerant at the evaporator temperature. The inlet to the compressor (State 1) is set at the evaporator exit pressure. Superheat can be considered in the evaporator to prevent condensation in the compressor; however it is neglected in this single stage R-134a cycle model. The following equations are used to fully define the compressor inlet state, beginning with the inlet temperature:

$$
\mathrm{T}_{1}=\mathrm{T}_{\text {chilled water return }}-\Delta \mathrm{T}_{\text {approach }}
$$

All of the other state properties for the evaporator exit/compressor inlet are calculated assuming the refrigerant is saturated at the temperature, $\mathrm{T}_{1}$. These properties are calculated using the internal EES property relations for R-134a (Tillner-Roth and Baehr, 1994).

$$
\begin{array}{r}
\mathrm{p}_{1}=\operatorname{pressure}\left(\mathrm{R} 134 \mathrm{a}, \mathrm{T}=\mathrm{T}_{1}, \text { quality }=1\right) \\
\mathrm{h}_{1}=\operatorname{enthalpy}\left(\mathrm{R} 134 \mathrm{a}, \mathrm{T}=\mathrm{T}_{1}, \text { quality }=1\right) \\
\mathrm{s}_{1}=\operatorname{entropy}\left(\mathrm{R} 134 \mathrm{a}, \mathrm{T}=\mathrm{T}_{1}, \text { quality }=1\right) \\
\mathrm{V}_{1}=\operatorname{volume}\left(\mathrm{R} 134 \mathrm{a}, \mathrm{T}=\mathrm{T}_{1}, \text { quality }=1\right)
\end{array}
$$

The refrigerant is approximated as being stagnant (i.e., zero velocity) at the compressor inlet, thus the stagnation and static properties are identical.

$$
\mathrm{p}_{\mathrm{o}, 1}=\mathrm{p}_{1} ; \mathrm{T}_{\mathrm{o}, 1}=\mathrm{T}_{1} ; \mathrm{h}_{\mathrm{o}, 1}=\mathrm{h}_{1} ; \mathrm{s}_{\mathrm{o}, 1}=\mathrm{s}_{1}
$$

If the inlet velocity were non-zero then the stagnation enthalpy would be defined instead by Equation (4.5), where $\mathrm{c}_{1}$ is the refrigerant velocity at the inlet.

$$
\mathrm{h}_{\mathrm{o}, 1}=\mathrm{h}_{1}+\frac{\mathrm{c}_{1}^{2}}{2}
$$

The compressor model establishes intermediate state properties by iterating toward the desired pressure ratio through variation of the overall compressor isentropic 
efficiency. The assumed isentropic efficiency relates compressor inlet enthalpy $\left(\mathrm{h}_{\mathrm{o}, 1}\right)$, the compressor exit isentropic stagnation enthalpy $\left(\mathrm{h}_{\mathrm{o}, 6 \mathrm{~s}}\right)$, and the actual compressor exit stagnation enthalpy $\left(\mathrm{h}_{\mathrm{o}, 6}\right)$. The exit stagnation enthalpy is determined here based on the assumed efficiency and again at the end of the iteration process. Only when the value of the exit stagnation enthalpy predicted by the model matches the required value is the iteration process complete.

$$
\eta_{\mathrm{c}}=\frac{\mathrm{h}_{\mathrm{o}, 6 \mathrm{~s}}-\mathrm{h}_{\mathrm{o}, 1}}{\mathrm{~h}_{\mathrm{o}, 6}-\mathrm{h}_{\mathrm{o}, 1}}
$$

The isentropic exit stagnation enthalpy is defined.

$$
\mathrm{h}_{\mathrm{o}, 6 \mathrm{~s}}=\text { enthalpy }\left(\mathrm{R} 134 \mathrm{a}, \mathrm{p}=\mathrm{r} \cdot \mathrm{p}_{\mathrm{o}, 1}, \mathrm{~s}=\mathrm{s}_{1}\right)
$$

The required change in stagnation enthalpy from compressor inlet to exit $\left(\Delta \mathrm{h}_{\mathrm{o}}\right)$ is used in many later calculations.

$$
\Delta \mathrm{h}_{\mathrm{o}}=\mathrm{h}_{\mathrm{o}, 6}-\mathrm{h}_{\mathrm{o}, 1}
$$

The impeller tip tangential velocity $\left(\mathrm{u}_{\mathrm{tip}}\right)$ is known from Euler's relationship for angular momentum according to the slip factor, $\sigma$, and the change in stagnation enthalpy, $\Delta \mathrm{h}_{\mathrm{o}}$.

$$
\mathrm{u}_{\text {tip }}=\sqrt{\frac{\Delta \mathrm{h}_{\mathrm{o}}}{\sigma}}
$$

The first process that the refrigerant will undergo upon entering the centrifugal compressor is acceleration through a nozzle from state (1) to state (2). No work is imparted to the refrigerant, however the nozzle is not ideal and therefore the process generates entropy. The stagnation enthalpy remains constant, but the stagnation pressure 
and temperature drop, while the stagnation entropy rises. The stagnation enthalpies of states $(0,1)$ and $(0,2)$ are set equal.

$$
\mathrm{h}_{\mathrm{o}, 2}=\mathrm{h}_{\mathrm{o}, 1}
$$

The nozzle exit velocity is set at $35 \%$ of the tip velocity, as shown by Equation (4.11). The 0.35 factor ensures that the average impeller inlet blade angle is slightly less than 70 deg. which is near optimal based on minimizing the average relative velocity of the fluid with respect to the impeller (this is the velocity that scales the kinetic energy in the impeller and therefore dictates many of the dominant losses in the compressor).

$$
\mathrm{c}_{2}=0.35 \cdot \mathrm{u}_{\mathrm{tip}}
$$

The nozzle exit enthalpy is related to the stagnation enthalpy and the absolute refrigerant velocity.

$$
\mathrm{h}_{2}=\mathrm{h}_{\mathrm{o}, 2}+\frac{\mathrm{c}_{2}^{2}}{2}
$$

The acceleration of the refrigerant through the nozzle is accompanied by losses such as viscous shear and separation. These losses are estimated using an efficiency value for converging nozzles $\left(\eta_{\text {nozzle }}\right)$. With the nozzle efficiency specified and the static exit enthalpy determined from Equation (4.12), the isentropic stagnation enthalpy is determined in Equation (4.13). This is used to determine the pressure at the nozzle exit.

$$
\eta_{\text {nozzle }}=\frac{\mathrm{h}_{\mathrm{o}, 2}-\mathrm{h}_{2}}{\mathrm{~h}_{\mathrm{o}, 2 \mathrm{~s}}-\mathrm{h}_{2}}
$$

The pressure is determined using the nozzle exit isentropic stagnation enthalpy and the entropy at nozzle inlet.

$$
\mathrm{p}_{2}=\operatorname{pressure}\left(\mathrm{R} 134 \mathrm{a}, \mathrm{h}=\mathrm{h}_{\mathrm{o}, 2 \mathrm{~s}}, \mathrm{~s}=\mathrm{s}_{1}\right)
$$


All of the other static state properties for the nozzle exit are calculated using EES built-in thermophysical property functions, with the enthalpy and pressure known.

$$
\begin{array}{r}
\mathrm{T}_{2}=\text { temperature }\left(\mathrm{R} 134 \mathrm{a}, \mathrm{p}=\mathrm{p}_{2}, \mathrm{~h}=\mathrm{h}_{2}\right) \\
\mathrm{s}_{2}=\operatorname{entropy}\left(\mathrm{R} 134 \mathrm{a}, \mathrm{p}=\mathrm{p}_{2}, \mathrm{~h}=\mathrm{h}_{2}\right) \\
\mathrm{v}_{2}=\operatorname{volume}\left(\mathrm{R} 134 \mathrm{a}, \mathrm{p}=\mathrm{p}_{2}, \mathrm{~h}=\mathrm{h}_{2}\right) \\
\mathrm{x}_{2}=\text { quality }\left(\mathrm{R} 134 \mathrm{a}, \mathrm{p}=\mathrm{p}_{2}, \mathrm{~h}=\mathrm{h}_{2}\right)
\end{array}
$$

The nozzle exit stagnation properties are calculated using the stagnation enthalpy and entropy.

$$
\begin{aligned}
& \mathrm{s}_{\mathrm{o}, 2}=\mathrm{s}_{1} \\
& \mathrm{~T}_{\mathrm{o}, 2}=\operatorname{temperature}\left(\mathrm{R} 134 \mathrm{a}, \mathrm{h}=\mathrm{h}_{\mathrm{o}, 2}, \mathrm{~s}=\mathrm{s}_{\mathrm{o}, 2}\right) \\
& \mathrm{p}_{\mathrm{o}, 2}=\operatorname{pressure}\left(\mathrm{R} 134 \mathrm{a}, \mathrm{h}=\mathrm{h}_{\mathrm{o}, 2}, \mathrm{~s}=\mathrm{s}_{\mathrm{o}, 2}\right)
\end{aligned}
$$

The second process that the refrigerant undergoes is a work input via the impeller blade, from state (2) to state (3). The impeller rotational velocity $(\Omega)$ is calculated using the definition of the specific speed $\left(\mathrm{n}_{\mathrm{s}}\right)($ Balje, 1981).

$$
\Omega=\frac{\mathrm{n}_{\mathrm{s}} \cdot \Delta \mathrm{h}_{\mathrm{o}}^{3 / 4}}{\sqrt{\dot{\mathrm{m}} \cdot \mathrm{v}_{1}}}
$$

The tip radius $\left(\mathrm{r}_{\text {tip }}\right)$ is calculated using the impeller rotational velocity and the tip speed.

$$
\mathrm{r}_{\text {tip }}=\frac{\mathrm{u}_{\text {tip }}}{\Omega}
$$

The flow enters the impeller in the axial direction. The hub radius $\left(\mathrm{r}_{\text {hub }}\right)$ is defined by the hub-to-tip radius ratio ( $\mathrm{r}_{\text {hub-to-tip }}$ ), which is specified as a model input parameter.

$$
\mathrm{r}_{\text {hub }}=\mathrm{r}_{\text {tip }} \cdot \mathrm{r}_{\text {hub-to-tip }}
$$

The shroud radius ( $\mathrm{r}_{\text {shroud }}$ ) is found using mass conservation.

$$
\mathrm{r}_{\text {shroud }}=\sqrt{\mathrm{r}_{\text {hub }}^{2}+\frac{\dot{\mathrm{m}} \cdot \mathrm{v}_{2}}{\mathrm{c}_{2} \cdot \pi}}
$$


Using velocity vector diagrams as a visual tool, it is possible to determine the velocity relative to the impeller blade $\left(\mathrm{w}_{2}\right)$, the absolute velocity $\left(\mathrm{c}_{2}\right)$, and the impeller blade velocity $\left(\mathrm{u}_{2}\right)$ at both the hub (subscript ' $\mathrm{h}$ ') and the shroud (subscript 's'). Characteristic hub and shroud velocity vector diagrams are shown in Figure 33.

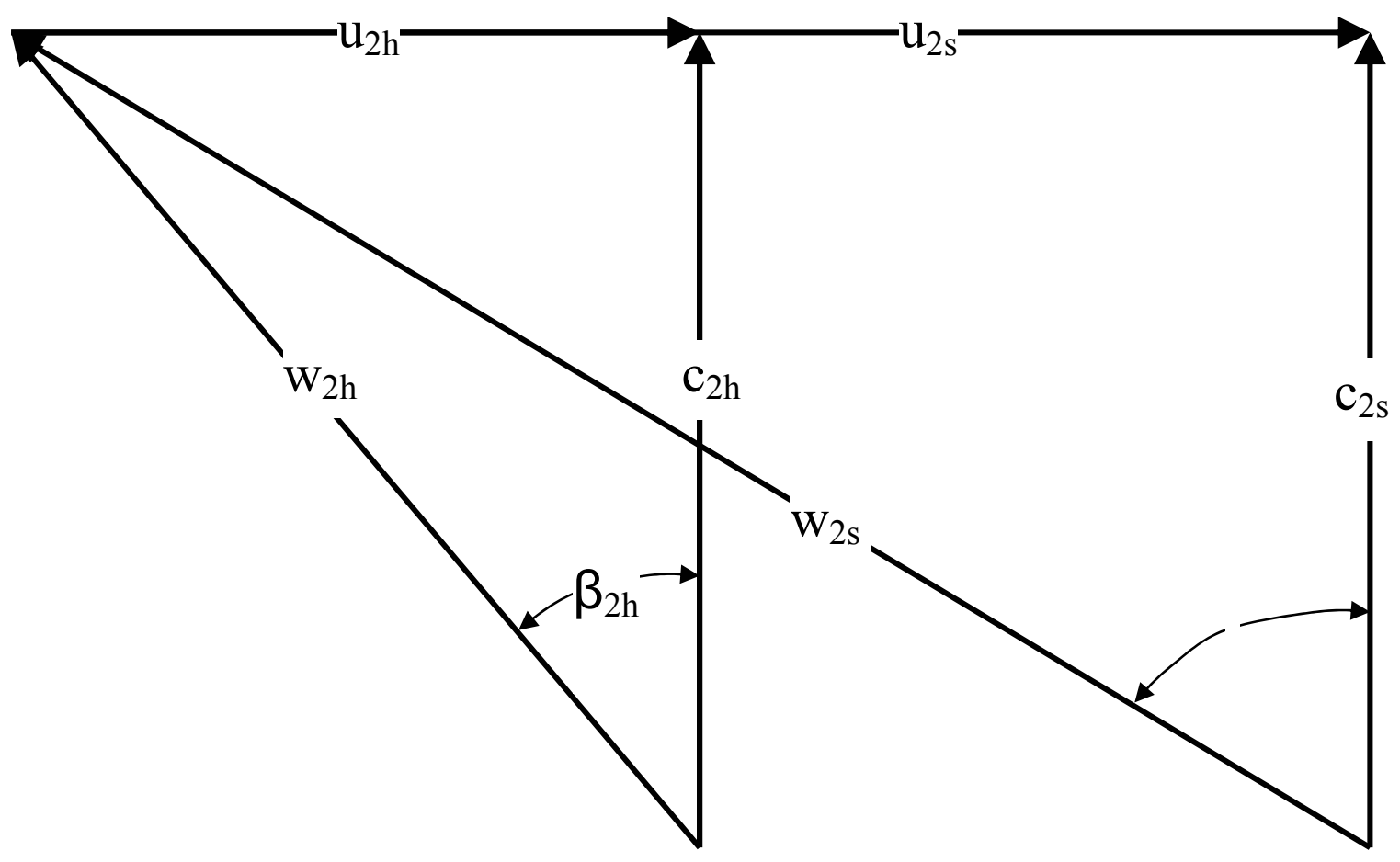

Figure 33 Velocity vector diagrams for the impeller blade hub and shroud inlets.

The impeller tangential velocities at the hub ( $\mathrm{u}_{\text {hub }}$ ) and at the shroud ( $\mathrm{u}_{\text {shroud }}$ ) are calculated using the associated impeller blade radii and the rotational velocity.

$$
\begin{gathered}
\mathrm{u}_{\text {hub }}=\mathrm{r}_{\text {hub }} \cdot \Omega \\
\mathrm{u}_{\text {shroud }}=\mathrm{r}_{\text {shroud }} \cdot \Omega
\end{gathered}
$$

The impeller inlet blade angles are set such that there is zero fluid impingement on the blades, which is a source of loss. The impeller inlet blade angles vary from the hub $\left(\beta_{\text {hub }}\right)$ to the shroud $\left(\beta_{\text {shroud }}\right)$ due to the difference between the impeller blade 
tangential velocities from the hub to the shroud. These blade angles are calculated according to:

$$
\begin{aligned}
\beta_{\text {hub }} & =\arctan \left(\frac{\mathrm{u}_{\text {hub }}}{\mathrm{c}_{2}}\right) \\
\beta_{\text {shroud }} & =\arctan \left(\frac{\mathrm{u}_{\text {shroud }}}{\mathrm{c}_{2}}\right)
\end{aligned}
$$

Next, the compressor model calculates the refrigerant velocity relative to the inlet shroud ( $\mathrm{w}_{\text {shroud }}$ ) in Equation (4.25). If the relative Mach number is too large (i.e. greater than about 0.8 ), then the relative velocity will be set equal to a fraction of the speed of sound at that state.

$$
\mathrm{w}_{\text {shroud }}=\sqrt{\mathrm{u}_{\text {shroud }}^{2}+\mathrm{c}_{2}^{2}}
$$

The Mach number relative to the shroud $\left(\mathrm{Ma}_{\mathrm{r}, \mathrm{shroud}}\right)$ is found using EES built-in functions for sound speed evaluated at state (2) temperature and pressure.

$$
\mathrm{Ma}_{\mathrm{r}, \text { shroud }}=\frac{\mathrm{w}_{\text {shroud }}}{\text { soundspeed }\left(\mathrm{R} 134 \mathrm{a}, \mathrm{T}=\mathrm{T}_{2}, \mathrm{p}=\mathrm{p}_{2}\right)}
$$

The absolute Mach number at the shroud $\left(\mathrm{Ma}_{\text {shroud }}\right)$ is calculated in the same manner as the relative Mach number at the shroud.

$$
\mathrm{Ma}_{\text {shroud }}=\frac{\mathrm{c}_{2}}{\text { soundspeed(R134a, } \left.\mathrm{T}=\mathrm{T}_{2}, \mathrm{p}=\mathrm{p}_{2}\right)}
$$

Along the impeller blade, momentum diffusion will occur which will slow the relative velocity of the flow. The deceleration of the flow in the direction of adverse pressure gradient will tend to cause separation. The diffusion is defined as the ratio of the relative velocity at the tip to the relative velocity at the shroud. If the diffusion is 
maintained above a certain level, then separation can be avoided. This requirement is enforced by:

$$
\mathrm{w}_{\text {tip }}=\text { diffusion } \cdot \mathrm{w}_{\text {shroud }}
$$

The refrigerant leaving the impeller will not exactly follow the blade due to its inertia. Instead, the refrigerant deviates or "slips" between the impeller and the shroud. This is accounted for by the slip factor, which is the deviation between the tangential velocities that the fluid has to the tangential velocity it would have if it perfectly followed the blade. Equation (4.29) shows how the relative velocity is affected by slip at the impeller tip, where $c_{\text {slip }}$ is subtracted from the relative velocity at the impeller exit.

$$
\mathrm{c}_{\text {slip }}=(1-\sigma) \cdot \mathrm{u}_{\text {tip }}
$$

Equation (4.30) shows how the absolute velocity $\left(c_{3}\right)$ is affected by slip and indirectly by the diffusion.

$$
\mathrm{c}_{3}=\sqrt{\left(\mathrm{w}_{\text {tip }}^{2}-\mathrm{c}_{\text {slip }}^{2}\right)+\mathrm{u}_{\mathrm{tip}}^{2} \cdot \sigma^{2}}
$$

The compressor model determines the impeller exit stagnation state properties. The actual impeller exit stagnation enthalpy $\left(\mathrm{h}_{0,3}\right)$ is known because it is equal to the stagnation enthalpy at the compressor exit $\left(\mathrm{h}_{\mathrm{o}, 6}\right)$, which was calculated in Equation (4.6). Given an impeller total-to-total efficiency, the isentropic compressor exit enthalpy can be determined from the following equation.

$$
\mathrm{h}_{\mathrm{o}, 3 \mathrm{~s}}=\mathrm{h}_{\mathrm{o}, 2}+\eta_{\mathrm{TT}, \text { imp }} \cdot\left(\mathrm{h}_{\mathrm{o}, 3}-\mathrm{h}_{\mathrm{o}, 2}\right)
$$

Although the static pressure of the fluid at the impeller exit is increased relative to the suction pressure, this is not the final compressor exit pressure. An additional static 
pressure rise can be recovered in a series of downstream diffusers. The impeller exit stagnation pressure is defined by the following equation.

$$
\mathrm{p}_{\mathrm{o}, 3}=\operatorname{pressure}\left(\mathrm{R} 134 \mathrm{a}, \mathrm{h}=\mathrm{h}_{\mathrm{o}, 3 \mathrm{~s}}, \mathrm{~s}=\mathrm{s}_{2}\right)
$$

The remaining stagnation state properties are determined using the pressure and the enthalpy.

$$
\begin{aligned}
& \mathrm{T}_{\mathrm{o}, 3}=\text { temperature }\left(\mathrm{R} 134 \mathrm{a}, \mathrm{p}=\mathrm{p}_{\mathrm{o}, 3}, \mathrm{~h}=\mathrm{h}_{\mathrm{o}, 3}\right) \\
& \mathrm{s}_{\mathrm{o}, 3}=\operatorname{entropy}\left(\mathrm{R} 134 \mathrm{a}, \mathrm{p}=\mathrm{p}_{\mathrm{o}, 3}, \mathrm{~h}=\mathrm{h}_{\mathrm{o}, 3}\right)
\end{aligned}
$$

The static state properties at the impeller exit are determined using the relationship between the stagnation enthalpy and the absolute velocity and the fact that the stagnation entropy is equal to the static entropy.

$$
\begin{gathered}
\mathrm{h}_{3}=\mathrm{h}_{\mathrm{o}, 3}-\frac{\mathrm{c}_{3}^{2}}{2} \\
\mathrm{~s}_{3}=\mathrm{s}_{0,3}
\end{gathered}
$$

The remaining static state properties are calculated using the static enthalpy and entropy at the impeller exit.

$$
\begin{array}{r}
\mathrm{p}_{3}=\operatorname{pressure}\left(\mathrm{R} 134 \mathrm{a}, \mathrm{h}=\mathrm{h}_{3}, \mathrm{~s}=\mathrm{s}_{3}\right) \\
\mathrm{T}_{3}=\operatorname{temperature}\left(\mathrm{R} 134 \mathrm{a}, \mathrm{h}=\mathrm{h}_{3}, \mathrm{~s}=\mathrm{s}_{3}\right) \\
\mathrm{v}_{3}=\operatorname{volume}\left(\mathrm{R} 134 \mathrm{a}, \mathrm{h}=\mathrm{h}_{3}, \mathrm{~s}=\mathrm{s}_{3}\right)
\end{array}
$$

Under normal operating conditions, the absolute Mach numbers at the impeller inlet are low. At the exit of the impeller, the Mach numbers can approach unity or higher, particularly if a high pressure ratio is required. This is an adverse condition for recovering static pressure since shock losses result in low coefficients of pressure recovery. These shock losses can be avoided using a gradual deceleration to subsonic speeds through a vaneless space. The third process the refrigerant undergoes is therefore 
static pressure recovery through a vaneless diffuser. In operating conditions where the impeller Mach number is greater than 0.8 , the compressor model assumes that a vaneless space exists. The extent of the vaneless space depends on the Mach number at the impeller exit. The Mach number at the impeller exit $\left(\mathrm{Ma}_{\text {tip }}\right)$ is calculated by:

$$
\mathrm{Ma}_{\text {tip }}=\frac{\mathrm{c}_{3}}{\text { soundspeed }\left(\mathrm{R} 134 \mathrm{a}, \mathrm{T}=\mathrm{T}_{3}, \mathrm{p}=\mathrm{p}_{3}\right)}
$$

The Mach number relative to the impeller tip $\left(\mathrm{Ma}_{\mathrm{r}, \mathrm{tip}}\right)$ is calculated by:

$$
\mathrm{Ma}_{\mathrm{r}, \text { tip }}=\frac{\mathrm{w}_{\text {tip }}}{\operatorname{soundspeed}\left(\mathrm{R} 134 \mathrm{a}, \mathrm{T}=\mathrm{T}_{3}, \mathrm{p}=\mathrm{p}_{3}\right)}
$$

Using conservation of angular momentum and vector diagrams, the components of the refrigerant velocity exiting the impeller blade can be found using the following equations, where the absolute tangential velocity is $\mathrm{c}_{\theta, 3}$ and the absolute meanline velocity is $\mathrm{c}_{\text {mean,3. }}$.

$$
\begin{aligned}
& \mathrm{c}_{\theta, 3}=\mathrm{u}_{\text {tip }}-\mathrm{c}_{\text {slip }} \\
& \mathrm{c}_{\text {mean }, 3}=\sqrt{\mathrm{w}_{\text {tip }}^{2}-\mathrm{c}_{\text {slip }}^{2}}
\end{aligned}
$$

The angle at which the refrigerant leaves the impeller tip $\left(\alpha_{\text {tip }}\right)$ can be calculated using the absolute velocities calculated above.

$$
\tan \left(\alpha_{\text {tip }}\right)=\frac{\mathrm{c}_{\theta, 3}}{\mathrm{c}_{\text {mean }, 3}}
$$

The compressor model now calculates the vaneless space exit stagnation properties. The stagnation enthalpy $\left(\mathrm{h}_{\mathrm{o}, 4}\right)$ remains unchanged.

$$
\mathrm{h}_{\mathrm{o}, 4}=\mathrm{h}_{\mathrm{o}, 3}
$$


The stagnation pressure at the vaneless space exit $\left(\mathrm{p}_{\mathrm{o}, 4}\right)$ is related to the stagnation pressure at state (3), by the vaneless space efficiency. This is shown in Equation (4.42).

$$
\mathrm{p}_{\mathrm{o}, 4}=\mathrm{p}_{\mathrm{o}, 3} \cdot \eta_{\mathrm{vs}}
$$

The stagnation temperature $\left(\mathrm{T}_{0,4}\right)$ and entropy $\left(\mathrm{s}_{0,4}\right)$ are:

$$
\begin{array}{r}
\mathrm{T}_{\mathrm{o}, 4}=\operatorname{temperature}\left(\mathrm{R} 134 \mathrm{a}, \mathrm{p}=\mathrm{p}_{\mathrm{o}, 4}, \mathrm{~h}=\mathrm{h}_{\mathrm{o}, 4}\right) \\
\mathrm{s}_{\mathrm{o}, 4}=\operatorname{entropy}\left(\mathrm{R} 134 \mathrm{a}, \mathrm{p}=\mathrm{p}_{\mathrm{o}, 4}, \mathrm{~h}=\mathrm{h}_{\mathrm{o}, 4}\right)
\end{array}
$$

The velocity at the vaneless space exit is set at a fraction of sound speed in order to avoid shock. For the R-134a compressor, the Mach number is set to be 0.8 as a design condition for the vaneless space exit. The Mach number $\left(\mathrm{Ma}_{4}\right)$ is calculated in the following equation to determine the refrigerant exit velocity $\left(\mathrm{c}_{4}\right)$.

$$
\mathrm{Ma}_{4}=\frac{\mathrm{c}_{4}}{\text { soundspeed }\left(\mathrm{R} 134 \mathrm{a}, \mathrm{h}=\mathrm{h}_{4}, \mathrm{~s}=\mathrm{s}_{4}\right)}
$$

The compressor model determines the static state of the vaneless space exit. The static enthalpy $\left(h_{4}\right)$ can be found using the refrigerant velocity and the stagnation enthalpy.

$$
\mathrm{h}_{4}=\mathrm{h}_{\mathrm{o}, 4}-\mathrm{c}_{4}
$$

The static entropy $\left(s_{4}\right)$ is equal to the stagnation entropy at the vaneless space exit.

$$
\mathrm{S}_{4}=\mathrm{S}_{\mathrm{o}, 4}
$$

The remaining static properties are calculated in the following equations using the static enthalpy and entropy.

$$
\begin{array}{r}
\mathrm{T}_{4}=\operatorname{temperature}\left(\mathrm{R} 134 \mathrm{a}, \mathrm{h}=\mathrm{h}_{4}, \mathrm{~s}=\mathrm{s}_{4}\right) \\
\mathrm{p}_{4}=\operatorname{pressure}\left(\mathrm{R} 134 \mathrm{a}, \mathrm{h}=\mathrm{h}_{4}, \mathrm{~s}=\mathrm{s}_{4}\right) \\
\mathrm{v}_{4}=\operatorname{volume}\left(\mathrm{R} 134 \mathrm{a}, \mathrm{h}=\mathrm{h}_{4}, \mathrm{~s}=\mathrm{s}_{4}\right)
\end{array}
$$


The components of the vaneless space exit velocity can be calculated using conservation of angular momentum and vector diagrams. The relationship between the angle the refrigerant leaves the vaneless space $\left(\alpha_{4}\right)$ to the angle it leaves from the impeller tip is:

$$
\tan \left(\alpha_{\text {tip }}\right) \cdot \mathrm{v}_{3}=\tan \left(\alpha_{4}\right) \cdot \mathrm{v}_{4}
$$

The angle the refrigerant leaves the vaneless space relates the components of the absolute refrigerant velocity, $\mathrm{c}_{\theta, 4}$ and $\mathrm{c}_{\text {mean, }, 4}$, in the following relationship.

$$
\tan \left(\alpha_{4}\right)=\frac{\mathrm{c}_{\theta, 4}}{\mathrm{c}_{\text {mean }, 4}}
$$

The sum of these components yields the absolute velocity at the vaneless space exit.

$$
\mathrm{c}_{4}=\sqrt{\mathrm{c}_{\theta, 4}^{2}+\mathrm{c}_{\text {mean }, 4}^{2}}
$$

The fourth process the refrigerant undergoes is the static pressure recovery through a vaned diffuser. In the diffuser, the velocity of the gas is reduced in order to elevate the static pressure. The design of an efficient diffuser is complex because gas is moving against the pressure gradient. If the deceleration of the flow is too rapid, flow separation may result disrupting the process of converting kinetic energy into pressure energy. Flow separation is an undesirable characteristic in diffusers because it leads to large stagnation pressure losses. Figure 34 shows a diffuser and illustrates the velocity profile of flow in the regimes before separation, on the verge of separation, and at separation. 


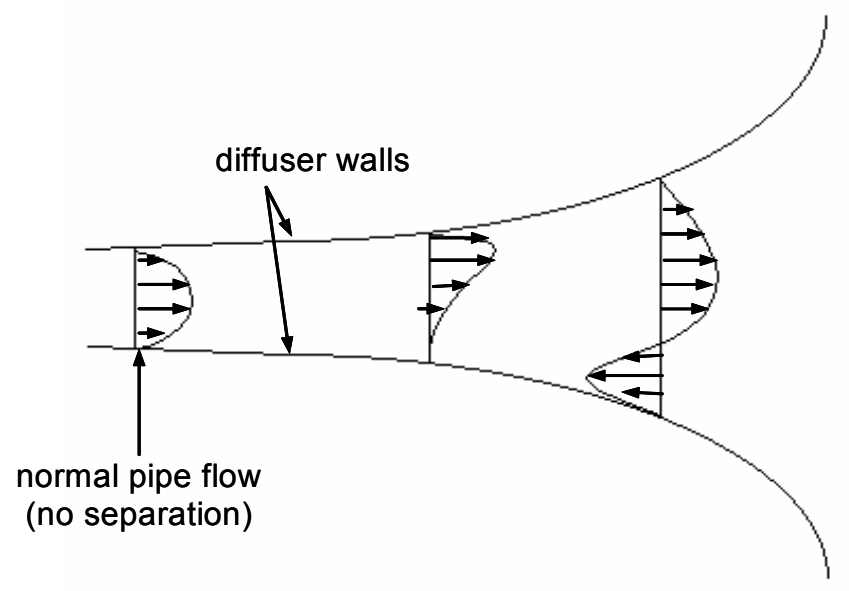

Figure 34 Vaned diffuser velocity profiles.

The coefficient of pressure recovery $\left(\mathrm{C}_{\mathrm{pr}}\right)$ is a parameter that is used to quantify the performance of the vaned diffuser.

$$
\mathrm{p}_{5}=\mathrm{C}_{\mathrm{pr}} \cdot\left(\mathrm{p}_{\mathrm{o}, 4}-\mathrm{p}_{4}\right)+\mathrm{p}_{4}
$$

The static enthalpy at state (5) is related to the stagnation enthalpy of the vaneless space exit $\left(\mathrm{h}_{\mathrm{o}, 4}\right)$ and the refrigerant velocity $\left(\mathrm{c}_{5}\right)$.

$$
\mathrm{h}_{5}=\mathrm{h}_{\mathrm{o}, 4}-\mathrm{c}_{5}
$$

The refrigerant velocity at the exit of the vaned diffuser is calculated using a specified velocity ratio $\left(\mathrm{c}_{5} / \mathrm{c}_{4}\right)$. The velocity ratio is similar to the diffusion parameter used in the impeller in that it is set at a reasonable value in order to avoid separation losses.

$$
\mathrm{c}_{5}=\mathrm{c}_{4} \cdot \mathrm{c}_{5} / \mathrm{c}_{4}
$$

The remaining static state properties are calculated.

$$
\begin{array}{r}
\mathrm{s}_{5}=\operatorname{entropy}\left(\mathrm{R} 134 \mathrm{a}, \mathrm{p}=\mathrm{p}_{5}, \mathrm{~h}=\mathrm{h}_{5}\right) \\
\mathrm{T}_{5}=\operatorname{temperature}\left(\mathrm{R} 134 \mathrm{a}, \mathrm{p}=\mathrm{p}_{5}, \mathrm{~h}=\mathrm{h}_{5}\right) \\
\mathrm{v}_{5}=\operatorname{volume}\left(\mathrm{R} 134 \mathrm{a}, \mathrm{p}=\mathrm{p}_{5}, \mathrm{~h}=\mathrm{h}_{5}\right)
\end{array}
$$

The stagnation enthalpy at the vaned diffuser exit is equated to the vaneless diffuser exit. 


$$
\mathrm{h}_{\mathrm{o}, 5}=\mathrm{h}_{\mathrm{o}, 4}
$$

The stagnation entropy at the vaned diffuser exit is equated to the static entropy at the same state.

$$
\mathrm{S}_{0,5}=\mathrm{S}_{5}
$$

The remaining stagnation properties for the vaned diffuser are calculated.

$$
\begin{array}{r}
\mathrm{T}_{\mathrm{o}, 5}=\text { temperature }\left(\mathrm{R} 134 \mathrm{a}, \mathrm{h}=\mathrm{h}_{\mathrm{o}, 5}, \mathrm{~s}=\mathrm{s}_{\mathrm{o}, 5}\right) \\
\mathrm{p}_{\mathrm{o}, 5}=\operatorname{pressure}\left(\mathrm{R} 134 \mathrm{a}, \mathrm{h}=\mathrm{h}_{\mathrm{o}, 5}, \mathrm{~s}=\mathrm{s}_{\mathrm{o}, 5}\right)
\end{array}
$$

The last process the refrigerant undergoes is collection into a large space; the final deceleration of the fluid prior to sending it on to the condenser. The refrigerant does not recover any more static pressure and the stagnation enthalpy remains constant. The refrigerant is assumed to be stagnant $\left(\mathrm{c}_{6}=0\right)$ in the collector (volute). The stagnation enthalpy is constant from the vaned diffuser to the volute.

$$
\mathrm{h}_{\mathrm{o}, 6}=\mathrm{h}_{\mathrm{o}, 5}
$$

The stagnation pressure at the volute exit is equated to the static pressure at the vaned diffuser exit.

$$
\mathrm{p}_{\mathrm{o}, 6}=\mathrm{p}_{5}
$$

The remaining stagnation state properties at the volute exit are calculated using the stagnation enthalpy and pressure in the following equations.

$$
\begin{array}{r}
\mathrm{s}_{\mathrm{o}, 6}=\operatorname{entropy}\left(\mathrm{R} 134 \mathrm{a}, \mathrm{p}=\mathrm{p}_{\mathrm{o}, 6}, \mathrm{~h}=\mathrm{h}_{\mathrm{o}, 6}\right) \\
\mathrm{T}_{\mathrm{o}, 6}=\operatorname{temperature}\left(\mathrm{R} 134 \mathrm{a}, \mathrm{p}=\mathrm{p}_{\mathrm{o}, 6}, \mathrm{~h}=\mathrm{h}_{\mathrm{o}, 6}\right)
\end{array}
$$

The static state properties are equated to the stagnation state properties of the volute. 


$$
\begin{aligned}
& \mathrm{T}_{6}=\mathrm{T}_{\mathrm{o}, 6} \\
& \mathrm{p}_{6}=\mathrm{p}_{\mathrm{o}, 6} \\
& \mathrm{~h}_{6}=\mathrm{h}_{\mathrm{o}, 6} \\
& \mathrm{~s}_{6}=\mathrm{s}_{\mathrm{o}, 6}
\end{aligned}
$$

After calculating $\mathrm{h}_{\mathrm{o}, 6}$ in Equation (4.61), the model compares the associated compressor efficiency with the initial guess. If they do not agree, then additional iterations occur until the efficiency calculation matches the initial guess.

\subsubsection{Size}

The result of the equations described above provides an estimate of the compressors size and performance. This section summarizes the size of a typical R-134a centrifugal compressor intended for a 1000-ton refrigeration cycle at the ARTI standard conditions.

Table 6 shows the size and operating speed of the R-134a centrifugal compressor.

Table 6 Dimensions and performance of predicted R-134a centrifugal compressor.

\begin{tabular}{l|l} 
Parameter & Value \\
\hline Tip Diameter & $12.23 \mathrm{inch}$ \\
Rotational speed & $10,000 \mathrm{rpm}$ \\
Pressure ratio & 2.578 \\
Specific Speed & $0.63 \mathrm{rad}$ \\
Isentropic efficiency & $82.5 \%$
\end{tabular}

Table 7 provides some measure of validation of the compressor model as it is capable of successfully predicting the size and performance of the centrifugal compressor used in today's conventional R-134a chiller. 
Table 7 Comparison of compressors and models in real applications with UW - Madison model.

\begin{tabular}{|c|c|c|c|c|c|c|c|c|c|}
\hline \multirow[b]{2}{*}{ 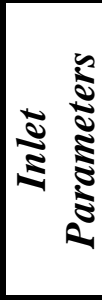 } & \multirow[b]{2}{*}{$\begin{array}{l}\text { Refrigerant } \\
\text { Inlet temp } \\
\text { Inlet pressure } \\
\text { Pressure ratio } \\
\text { Mass flow rate } \\
\text { Capacity }\end{array}$} & \multicolumn{2}{|c|}{ Water } & \multicolumn{2}{|c|}{$\mathrm{CCl}_{3} \mathrm{~F}(\mathrm{R}-11)$} & \multicolumn{2}{|c|}{$\mathrm{CCl}_{2} \mathrm{~F}_{2}(\mathrm{R}-12)$} & \multicolumn{2}{|c|}{$R 500$} \\
\hline & & \multicolumn{2}{|c|}{$\begin{array}{c}5.6^{\circ} \mathrm{C} \\
0.90 \mathrm{kPa} \\
6.60 \\
1.41 \mathrm{~kg} / \mathrm{s} \\
1000 \mathrm{ton}\end{array}$} & \multicolumn{2}{|c|}{$\begin{array}{c}2.0^{\circ} \mathrm{C} \\
40.2 \mathrm{kPa} \\
4.47 \\
6.89 \mathrm{~kg} / \mathrm{s} \\
300 \mathrm{ton}\end{array}$} & \multicolumn{2}{|c|}{$\begin{array}{c}2.0^{\circ} \mathrm{C} \\
319.8 \mathrm{kPa} \\
3.00 \\
10.23 \mathrm{~kg} / \mathrm{s} \\
300 \mathrm{ton}\end{array}$} & \multicolumn{2}{|c|}{$\begin{array}{c}11.4^{\circ} \mathrm{C} \\
418.5 \mathrm{kPa} \\
2.10 \\
42.24 \mathrm{~kg} / \mathrm{s} \\
1810 \mathrm{ton}\end{array}$} \\
\hline & Source & \begin{tabular}{|c|}
$\begin{array}{c}\text { Concepts } \\
\mathrm{ETI}^{1}\end{array}$ \\
\end{tabular} & $\begin{array}{c}\text { UW - } \\
\text { Madison } \\
\end{array}$ & \begin{tabular}{|l|} 
Wiesner, \\
Caswell $^{2}$ \\
\end{tabular} & \begin{tabular}{|c|}
$U W-$ \\
Madison \\
\end{tabular} & $\begin{array}{l}\text { Wiesner, } \\
\text { Caswell }^{2} \\
\end{array}$ & $\begin{array}{c}\text { UW - } \\
\text { Madison }\end{array}$ & $\begin{array}{l}\text { Braun, } \\
\text { D/F } W^{3} \\
\end{array}$ & $\begin{array}{c}\text { UW - } \\
\text { Madison }\end{array}$ \\
\hline \multirow{4}{*}{ 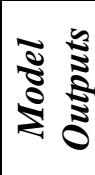 } & Rotation speed & $4800 \mathrm{rpm}$ & $5381 \mathrm{rpm}$ & $7610 \mathrm{rpm}$ & $6920 \mathrm{rpm}$ & $11870 \mathrm{rpm}$ & $10600 \mathrm{rpm}$ & $3900 \mathrm{rpm}$ & 3762 rpm \\
\hline & Efficiency (isen) & 0.747 & 0.764 & 0.766 & 0.763 & 0.780 & 0.749 & 0.697 & 0.708 \\
\hline & Tip radius & $1.33 \mathrm{~m}$ & $1.23 \mathrm{~m}$ & $0.24 m$ & $0.26 \mathrm{~m}$ & $0.09 \mathrm{~m}$ & $0.15 \mathrm{~m}$ & $0.36 \mathrm{~m}$ & $0.41 \mathrm{~m}$ \\
\hline & Tip velocity & $670 \mathrm{~m} / \mathrm{s}$ & $670 \mathrm{~m} / \mathrm{s}$ & $195 \mathrm{~m} / \mathrm{s}$ & $190 \mathrm{~m} / \mathrm{s}$ & $173 \mathrm{~m} / \mathrm{s}$ & $171 \mathrm{~m} / \mathrm{s}$ & $146 \mathrm{~m} / \mathrm{s}$ & $160 \mathrm{~m} / \mathrm{s}$ \\
\hline
\end{tabular}

1. Orsi, LeDrew, Wight, Yoshinaka, 2000, "The Efficiency Limits of Water Vapor Compressors", TM \# 762, Concepts ETI

2. Wiesner, Caswell, 1960, "How Refrigerant Properties Affect Impeller Dimensions", ASHRAE Journal, October, pp. 31-37.

3. Braun, 1988, "Methodologies for the Design and Control of Central Cooling Plants", Ph.D. Thesis, UWMadison.

\subsubsection{Cost}

The cost of a centrifugal compressor is estimated based on a combination of the fixed costs that go into the R\&D, labor, overhead together with the material and manufacturing costs that are assumed to be a function of the impeller tip diameter. In order to obtain an accurate estimate of the size and performance of the centrifugal compressor as well as the indirect contact heat exchangers described in subsequent sections, a survey was prepared and sent to several U.S. chiller manufacturers. The responses to this survey were averaged by ARI and provided as inputs for this project. For a 1000-ton R-134a chiller, the average cost of a compressor alone is found to be approximately $\$ 20,000$. The fixed cost is assumed to be approximately $40 \%$ of the total cost and the remaining cost accounted for in the materials. This is based on the assumption that these compressors are sold in high volume. The ability of this equation to predict the cost of a centrifugal compressor is discussed further in Chapter 5. The material cost is correlated to the tip diameter according to: 


$$
\text { MaterialCost }=\frac{\$ 25}{\text { in }^{2}} \cdot \mathrm{d}_{\text {tip }}^{2}+\frac{\$ 765}{\text { in }} \cdot \mathrm{d}_{\text {tip }}
$$

\subsubsection{Indirect-contact Condenser Model}

R-134a chillers employ indirect-contact heat exchangers (i.e. the secondary heat rejection fluid is not the same as the primary refrigerant). Here we assume a falling film condenser arranged in a shell-and-tube geometry as used in a conventional chiller, with water (the cooling medium) circulating in the tubes and refrigerant falling over the tubes in the shell. To account for surface enhancements, the governing equations for determining the heat transfer coefficients in the indirect-contact condenser model are calibrated such that the size and cost are approximately equivalent to an industry-average (based on the data supplied by ARI).

\subsubsection{Performance}

This section is a description of the indirect-contact heat exchanger model for the $\mathrm{R}-134 \mathrm{a}$ system. The model is developed in the EES environment and will ultimately be used to estimate the performance, size and cost of the typical shell and tube condenser that is appropriate for a 1000-ton refrigeration cycle. This is an iterative model that takes heat transfer and pressure loss into consideration when matching the heat exchanger dimensions with desired performance. Table 8 shows the user-specified parameters that contribute to the prediction of the heat exchanger design. 
Table 8 Indirect-contact condenser operating parameters.

\begin{tabular}{l|c|c} 
Parameter & Symbol & Default value \\
\hline Approach temperature & $\Delta \mathrm{T}_{\text {cond }}$ & varied \\
Condenser water velocity & $\mathrm{v}_{\mathrm{cw}}$ & $2.5 \mathrm{ft} / \mathrm{s}$ \\
Tubing material & $\mathrm{T} \$$ & 'Copper' \\
Tube pitch-to-tube OD ratio & Pitch $\$ tubeOD & 1.5 \\
Heat exchanger aspect ratio & $\mathrm{AR}$ & 5 \\
\hline Refrigerant mass flow rate & $\dot{\mathrm{m}}_{\mathrm{ref}}$ & Based on \\
Refrigerant inlet temperature & $\mathrm{T}_{1}$ & cycle \\
Condenser water inlet temperature & $\mathrm{T}_{3}$ & conditions \\
Condenser water exit temperature & $\mathrm{T}_{4}$ &
\end{tabular}

Figure 35 is a schematic of a shell and tube condenser similar to what would be seen in a typical R-134a chiller. The condenser water absorbs the latent heat of condensation as it flows through the tubes inside the shell. The refrigerant condenses as it passes through the surrounding shell. The condenser water temperature increases by $10^{\circ} \mathrm{F}$ as it passes through the tubes (from $85^{\circ} \mathrm{F}$ to $95^{\circ} \mathrm{F}$ ).

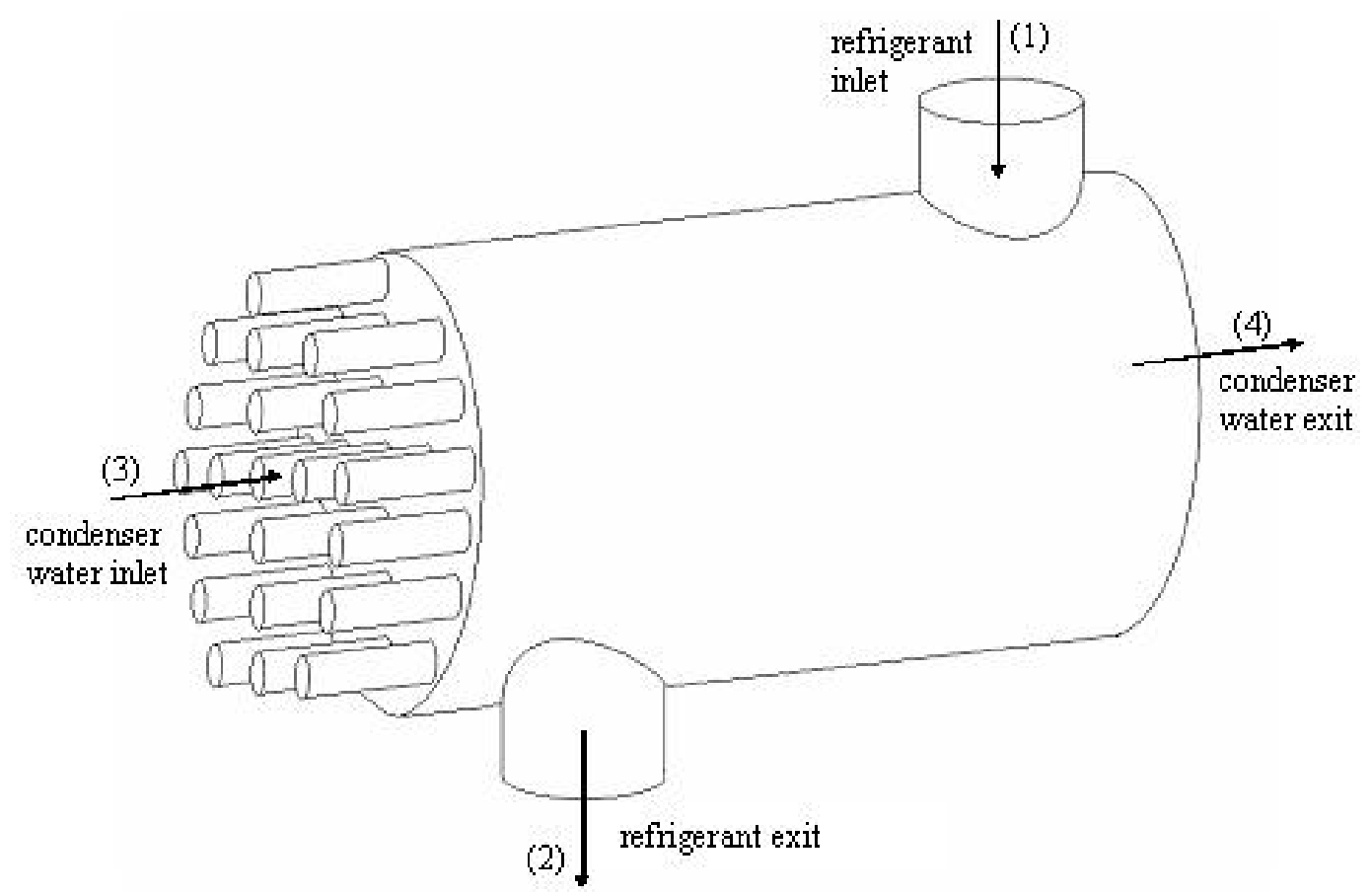

Figure 35 Schematic of indirect-contact condenser model with R-134a as a refrigerant. 
The size and performance of an indirect-contact heat exchanger are related; larger size will lead to better performance at the expense of higher capital cost. The inlet conditions for the shell-and-tube condenser model are set both by the exit parameters of the centrifugal compressor model (on the refrigerant side), and by ARTI specified condenser water conditions (on the water side). The state of the refrigerant at the inlet to the condenser is known from the centrifugal compressor model output.

$$
\begin{aligned}
& \text { (state 1) HX model Compressor model (state 6) } \\
& \mathrm{T}_{1}=\mathrm{T}_{6} \\
& \mathrm{p}_{1}=\mathrm{p}_{6} \\
& \mathrm{~s}_{1}=\mathrm{s}_{6} \\
& \mathrm{v}_{1}=\mathrm{v}_{6} \\
& \mathrm{~h}_{1}=\mathrm{h}_{6}
\end{aligned}
$$

State (2) in the heat exchanger is based on the heat transfer between the condenser water and the refrigerant and the refrigerant pressure loss. State (2) is defined before any of the dimensions are determined; the dimensions follow from this definition. Using the assumption that the refrigerant leaves the condenser a saturated liquid $\left(x_{2}=0\right)$, only the pressure at the condenser exit need be determined. The refrigerant pressure drop $\left(\Delta \mathrm{p}_{\text {ref,req }}\right)$ is specified and used to compute $\mathrm{p}_{2}$.

$$
\begin{aligned}
& \mathrm{x}_{2}=0 \\
& \mathrm{p}_{2}=\left(1-\Delta \mathrm{p}_{\text {ref,req }}\right) \cdot \mathrm{p}_{1}
\end{aligned}
$$

The pressure and the quality at the refrigerant side exit determine the remainder of the state properties.

$$
\begin{array}{r}
\mathrm{T}_{2}=\text { temperature }\left(\mathrm{R} 134 \mathrm{a}, \mathrm{p}=\mathrm{p}_{2}, \mathrm{x}=\mathrm{x}_{2}\right) \\
\mathrm{h}_{2}=\operatorname{enthalpy}\left(\mathrm{R} 134 \mathrm{a}, \mathrm{p}=\mathrm{p}_{2}, \mathrm{x}=\mathrm{x}_{2}\right) \\
\mathrm{s}_{2}=\operatorname{entropy}\left(\mathrm{R} 134 \mathrm{a}, \mathrm{p}=\mathrm{p}_{2}, \mathrm{x}=\mathrm{x}_{2}\right) \\
\mathrm{v}_{2}=\operatorname{volume}\left(\mathrm{R} 134 \mathrm{a}, \mathrm{p}=\mathrm{p}_{2}, \mathrm{x}=\mathrm{x}_{2}\right)
\end{array}
$$


The heat transfer rate is dependent on the temperature gradient from the condenser water to the refrigerant, therefore the condenser water states are determined first. Using the numbering convention from Figure 35, the ARTI standard temperature conditions for the condenser water entering and leaving the condenser are as follows.

$$
\begin{aligned}
\mathrm{T}_{3} & =85^{\circ} \mathrm{F} \\
\mathrm{T}_{4} & =95^{\circ} \mathrm{F}
\end{aligned}
$$

The pressure of the incoming condenser water, $\left(p_{3}\right)$, is assumed to be the sum of atmospheric pressure and the pressure required to overcome frictional losses $\left(\Delta \mathrm{p}_{\mathrm{cw}, \text { req }} \cdot \mathrm{p}_{4}\right.$ where $\Delta \mathrm{p}_{\mathrm{cw} \text {,req }}$ is the fraction of absolute pressure that is lost).

$$
\mathrm{p}_{3}=\left(1+\Delta \mathrm{p}_{\mathrm{cw}, \mathrm{req}}\right) \cdot \mathrm{p}_{4}
$$

The pressure and the temperature at the condenser water inlet determine the remainder of the state properties.

$$
\begin{array}{r}
\mathrm{h}_{3}=\operatorname{enthalpy}\left(\text { Water, } \mathrm{T}=\mathrm{T}_{3}, \mathrm{p}=\mathrm{p}_{3}\right) \\
\mathrm{s}_{3}=\operatorname{entropy}\left(\text { Water, } \mathrm{T}=\mathrm{T}_{3}, \mathrm{p}=\mathrm{p}_{3}\right) \\
\mathrm{v}_{3}=\operatorname{volume}\left(\text { Water, } \mathrm{T}=\mathrm{T}_{3}, \mathrm{p}=\mathrm{p}_{3}\right)
\end{array}
$$

The pressure at the condenser water exit, $\left(\mathrm{p}_{4}\right)$, is assumed to be atmospheric.

$$
\mathrm{p}_{4}=\mathrm{p}_{\mathrm{atm}}
$$

The pressure and temperature at the condenser water exit determine the remainder of the state properties.

$$
\begin{array}{r}
\mathrm{h}_{4}=\operatorname{enthalpy}\left(\text { Water, } \mathrm{T}=\mathrm{T}_{4}, \mathrm{p}=\mathrm{p}_{4}\right) \\
\mathrm{s}_{4}=\operatorname{entropy}\left(\text { Water, } \mathrm{T}=\mathrm{T}_{4}, \mathrm{p}=\mathrm{p}_{4}\right) \\
\mathrm{v}_{4}=\operatorname{volume}\left(\text { Water, } \mathrm{T}=\mathrm{T}_{4}, \mathrm{p}=\mathrm{p}_{4}\right)
\end{array}
$$

With all of the state properties known for the indirect-contact condenser, heat transfer and hydrodynamic effects must be considered in order to link the assumed 
performance with the required geometry. In order to evaluate the heat transfer coefficient for the condenser water as it passes through tubes, the average condenser water temperature $\left(\mathrm{T}_{\mathrm{cw}}\right)$ is required.

$$
\mathrm{T}_{\mathrm{cw}}=\frac{\mathrm{T}_{3}+\mathrm{T}_{4}}{2}
$$

The average pressure of the condenser water $\left(\mathrm{p}_{\mathrm{cw}}\right)$ from inlet to the exit is calculated.

$$
\mathrm{p}_{\mathrm{cw}}=\frac{\mathrm{p}_{3}+\mathrm{p}_{4}}{2}
$$

The constant pressure specific heat $\left(\mathrm{C}_{\mathrm{p}, \mathrm{cw}}\right)$, density $\left(\rho_{\mathrm{cw}}\right)$, viscosity $\left(\mu_{\mathrm{cw}}\right)$, thermal conductivity $\left(\mathrm{k}_{\mathrm{cw}}\right)$, and Prandtl number $\left(\mathrm{Pr}_{\mathrm{cw}}\right)$ of the condenser water are determined at the average temperature in the following equations, using EES built-in thermophysical property functions.

$$
\begin{array}{r}
\mathrm{C}_{\mathrm{p}, \mathrm{cw}}=\mathrm{cp}\left(\text { Water, } \mathrm{T}=\mathrm{T}_{\mathrm{cw}}, \mathrm{p}=\mathrm{p}_{\mathrm{cw}}\right) \\
\rho_{\mathrm{cw}}=\operatorname{density}\left(\text { Water, } \mathrm{T}=\mathrm{T}_{\mathrm{cw}}, \mathrm{p}=\mathrm{p}_{\mathrm{cw}}\right) \\
\mu_{\mathrm{cw}}=\operatorname{viscosity}\left(\text { Water, } \mathrm{T}=\mathrm{T}_{\mathrm{cw}}, \mathrm{p}=\mathrm{p}_{\mathrm{cw}}\right) \\
\mathrm{k}_{\mathrm{cw}}=\operatorname{conductivity}\left(\text { Water, } \mathrm{T}=\mathrm{T}_{\mathrm{cw}}, \mathrm{p}=\mathrm{p}_{\mathrm{cw}}\right) \\
\operatorname{Pr}_{\mathrm{cw}}=\operatorname{Prandtl}\left(\text { Water, } \mathrm{T}=\mathrm{T}_{\mathrm{cw}}, \mathrm{p}=\mathrm{p}_{\mathrm{cw}}\right)
\end{array}
$$

The tube is assumed to be a standard copper $3 / 4$ " OD tube and therefore the tube inner diameter $\left(\mathrm{d}_{\text {tube, }}\right)$ is:

$$
\left.\mathrm{d}_{\text {tube, } \mathrm{i}}=0.70 \text { [in }\right]
$$

The tube thickness ( $\mathrm{t}_{\text {tube }}$ ) is assumed to be a standard thickness for a $3 / 4$ " tube (McMasterCarr, 2003).

$$
\mathrm{t}_{\text {tube }}=0.025 \text { [in] }
$$


The shell is assumed to be baffled to allow the refrigerant to pass over the tubes more than once. The presence of the baffles affects only the vapor velocity and therefore the refrigerant pressure drop, which is not large in any case. The number of baffles $\left(\mathrm{N}_{\text {baffles }}\right)$ is user-specified. An unbaffled heat exchanger can be simulated by setting this parameter to 1 .

The conductance of the tube material is calculated using the thermal conductivity of copper $\left(\mathrm{k}_{\text {tube }}\right)$ evaluated at the average tube temperature. Thermal conductivity values of select solids are calculated using built-in EES functions.

$$
\mathrm{k}_{\text {tube }}=\mathrm{k}_{-}\left(\text {Copper, } \mathrm{T}_{\mathrm{cw}}\right)
$$

Refrigerant side properties are required to calculate heat transfer coefficients for condensation over a bank of tubes. Evaluation of heat transfer coefficients for two-phase flow typically requires saturated liquid and saturated vapor properties. The density of saturated liquid $\left(\rho_{1, \text { ref }}\right)$ and saturated vapor $\left(\rho_{\mathrm{v}, \mathrm{ref}}\right)$, thermal conductivity of saturated liquid $\left(\mathrm{k}_{1, \mathrm{ref}}\right)$, the viscosity of saturated liquid $\left(\mu_{1, \mathrm{ref}}\right)$ and saturated vapor $\left(\mu_{\mathrm{v}, \mathrm{ref}}\right)$, and the enthalpy of vaporization $\left(\mathrm{h}_{\mathrm{fg}, \text {,ref }}\right)$ of $\mathrm{R}-134 \mathrm{a}$ are evaluated at the exit temperature in the following equations.

$$
\begin{array}{r}
\rho_{1, \text { ref }}=\operatorname{density}\left(\mathrm{R} 134 \mathrm{a}, \mathrm{T}=\mathrm{T}_{2}, \mathrm{x}=0\right) \\
\rho_{\mathrm{v}, \text { ref }}=\operatorname{density}\left(\mathrm{R} 134 \mathrm{a}, \mathrm{T}=\mathrm{T}_{2}, \mathrm{x}=1\right) \\
\mathrm{k}_{\mathrm{l}, \text { ref }}=\text { conductivity }\left(\mathrm{R} 134 \mathrm{a}, \mathrm{T}=\mathrm{T}_{2}, \mathrm{x}=0\right) \\
\mu_{1, \text { ref }}=\operatorname{viscosity}\left(\mathrm{R} 134 \mathrm{a}, \mathrm{T}=\mathrm{T}_{2}, \mathrm{x}=0\right) \\
\mu_{\mathrm{v}, \text { ref }}=\operatorname{viscosity}\left(\mathrm{R} 134 \mathrm{a}, \mathrm{T}=\mathrm{T}_{2}, \mathrm{x}=1\right) \\
\mathrm{h}_{\mathrm{fg}, \text { ref }}=\text { enthalpy }\left(\mathrm{R} 134 \mathrm{a}, \mathrm{T}=\mathrm{T}_{2}, \mathrm{x}=1\right)-\mathrm{h}_{2}
\end{array}
$$

The final group of equations required to determine heat transfer coefficients and pressure drop are related to the tube bundle inside the heat exchanger shell. The tube outer diameter $\left(\mathrm{d}_{\text {tube,o }}\right)$ is a function of the tube inner diameter and the tube thickness. 


$$
\mathrm{d}_{\text {tube, } \mathrm{o}}=\mathrm{d}_{\text {tube, } \mathrm{i}}+2 \cdot \mathrm{t}_{\text {tube }}
$$

The distance between tube centers, or the tube pitch (pitch), is calculated using a design parameter that relates the tube outer diameter to the desired pitch (pitch $\backslash$ tubeOD). The pitch to tube outer diameter ratio is set based on the heat exchanger geometry data provided by manufacturers. If the refrigerant pressure loss is larger than a specified limit then the pitch can be increased.

$$
\text { pitch }=\text { pitch } \backslash \text { tubeOD } \cdot \mathrm{d}_{\text {tube,o }}
$$

The shell cross-sectional area $\left(\mathrm{A}_{\mathrm{HX}}\right)$ is related to the shell diameter $\left(\mathrm{D}_{\mathrm{HX}}\right)$.

$$
\mathrm{A}_{\mathrm{HX}}=\frac{\pi}{4} \cdot \mathrm{D}_{\mathrm{HX}}^{2}
$$

The number of tubes in the bundle enclosed by the cross-sectional area of the shell ( $\left.\mathrm{N}_{\text {tubes,Xsection }}\right)$ is calculated using a unit cell of the pattern.

$$
\mathrm{N}_{\text {tubes,Xsection }}=\frac{\mathrm{A}_{\mathrm{HX}}}{2 \cdot \mathrm{pitch}^{2} \cdot \sin \left(\frac{\pi}{3}\right) \cdot \cos \left(\frac{\pi}{3}\right)}
$$

The number of tubes that are vertically stacked in the bundle $\left(\mathrm{N}_{\text {tubes,vert }}\right)$ is calculated using the heat exchanger shell diameter and the tube pitch.

$$
\mathrm{N}_{\text {tubes, vert }}=\frac{\mathrm{D}_{\mathrm{HX}}}{\text { pitch }}
$$

The aspect ratio of the heat exchanger shell is not controlled well by the required pressure drop and so the aspect ratio is a design variable that can be independently set; practically this corresponds to a multiple tube pass heat exchanger. The aspect ratio (AR) is related to the heat exchanger length and diameter.

$$
\mathrm{AR}=\frac{\mathrm{L}_{\mathrm{HX}}}{\mathrm{D}_{\mathrm{HX}}}
$$


Because the heat exchanger typically has more than one tube-pass $\left(\mathrm{N}_{\text {pass }}\right)$, the total tube length $\left(\mathrm{L}_{\text {tube,total }}\right)$ is required to determine the condenser water side pressure drop.

$$
\mathrm{L}_{\text {tube,total }}=\mathrm{N}_{\text {pass }} \cdot \mathrm{L}_{\mathrm{HX}}
$$

The number of tubes in the shell cross-section is related to the number of tube-passes and the number of tubes.

$$
\mathrm{N}_{\text {tubes,Xsection }}=\mathrm{N}_{\text {pass }} \cdot \mathrm{N}_{\text {tubes }}
$$

The overall required conductance of the heat exchanger $\left(\mathrm{UA}_{\text {req }}\right)$ can be determined using the $\varepsilon$-NTU method. The required conductance is calculated using the following equations. The effectiveness $\left(\varepsilon_{\text {cond }}\right)$ is:

$$
\varepsilon_{\text {cond }}=\frac{\mathrm{T}_{4}-\mathrm{T}_{3}}{\mathrm{~T}_{2}-\mathrm{T}_{3}}
$$

The number of transfer units (NTU) is defined for a shell and tube heat exchanger with a zero capacitance ratio (i.e. $\mathrm{C}_{\mathrm{r}}=\frac{\dot{\mathrm{C}}_{\min }}{\dot{\mathrm{C}}_{\max }}=0$ ) by the following equation. Note that this approximation for the condenser neglects effects due to the refrigerant de-superheating region and the pressure loss; however, calculations show that these effects are small for the R-134a cycle.

$$
\mathrm{NTU}=-\ln \left(1-\varepsilon_{\text {cond }}\right)
$$

The required conductance is defined in terms of the NTU, the condenser water mass flow rate $\left(\dot{\mathrm{m}}_{\mathrm{cw}}\right)$, and the constant pressure specific heat of the condenser water.

$$
\mathrm{UA}_{\mathrm{req}}=\mathrm{NTU} \cdot \dot{\mathrm{m}}_{\mathrm{cw}} \cdot \mathrm{C}_{\mathrm{p}, \mathrm{cw}}
$$


The condenser water mass flow rate is determined by an energy balance between the refrigerant and the condenser water.

$$
\dot{\mathrm{m}}_{\mathrm{cw}} \cdot\left(\mathrm{h}_{4}-\mathrm{h}_{3}\right)=\dot{\mathrm{m}}_{\mathrm{ref}} \cdot\left(\mathrm{h}_{1}-\mathrm{h}_{2}\right)
$$

The fluid velocity $\left(\mathrm{v}_{\mathrm{cw}}\right)$ is required to determine the pressure drop as well as the Reynolds number for the heat transfer coefficient calculation. For this model, the fluid velocity is set to be $2.5 \mathrm{ft} / \mathrm{s}$, a value that balances the build up of solid contamination in the tubes resulting from sluggish flow against vibration and other effects of high velocity flow. This velocity determines the required number of tubes using conservation of mass through the tubes.

$$
\mathrm{v}_{\mathrm{cw}}=\frac{\dot{\mathrm{m}}_{\mathrm{cw}}}{\rho_{\mathrm{cw}} \cdot \mathrm{N}_{\text {tubes }} \cdot \frac{\pi}{4} \cdot \mathrm{d}_{\text {tube, } \mathrm{i}}^{2}}
$$

The condenser water Reynolds number is calculated.

$$
\operatorname{Re}_{\mathrm{cw}}=\frac{\rho_{\mathrm{cw}} \cdot \mathrm{v}_{\mathrm{cw}} \cdot \mathrm{d}_{\mathrm{tube}, \mathrm{i}}}{\mu_{\mathrm{cw}}}
$$

The condenser water friction factor $\left(\mathrm{f}_{\mathrm{cw}}\right)$ is calculated using a correlation for turbulent flow through smooth tubes (Incropera and DeWitt, 2002).

$$
\mathrm{f}_{\mathrm{cw}}=\left(0.79 \cdot \ln \left(\mathrm{Re}_{\mathrm{cw}}\right)-1.64\right)^{-2}
$$

This friction factor, along with the Reynolds and Prandtl numbers, is used to calculate the Nusselt number associated with the condenser water passing through the tubing. The condenser water Nusselt number $\left(\mathrm{Nu}_{\mathrm{cw}}\right)$ is calculated using a correlation for turbulent flow (Incropera and DeWitt, 2002). 


$$
\mathrm{Nu}_{\mathrm{cw}}=\frac{\left(\frac{\mathrm{f}_{\mathrm{cw}}}{8}\right) \cdot \operatorname{Re}_{\mathrm{cw}} \cdot \operatorname{Pr}_{\mathrm{cw}}}{1.07+12.7 \cdot\left(\frac{\mathrm{f}_{\mathrm{cw}}}{8}\right)^{1 / 2} \cdot\left(\operatorname{Pr}_{\mathrm{cw}}^{2 / 3}-1\right)}
$$

The definition of the Nusselt number leads to the calculation of the condenser water heat transfer coefficient.

$$
\mathrm{htc}_{\mathrm{cw}}=\frac{\mathrm{Nu}_{\mathrm{cw}} \cdot \mathrm{k}_{\mathrm{cw}}}{\mathrm{d}_{\text {tube, }}}
$$

The condenser water conductance $\left(\mathrm{UA}_{\mathrm{cw}}\right)$ is calculated using the heat transfer coefficient and the area over which heat transfer is taking place.

$$
\mathrm{UA}_{\mathrm{cw}}=\mathrm{htc}_{\mathrm{cw}} \cdot \mathrm{N}_{\text {tubes }} \cdot \pi \cdot \mathrm{d}_{\text {tube, } \mathrm{i}} \cdot \mathrm{L}_{\text {tube,total }}
$$

The condenser water side pressure $\operatorname{drop}\left(\Delta \mathrm{p}_{\mathrm{cw}}\right)$ is calculated using the friction factor.

$$
\Delta \mathrm{p}_{\mathrm{cw}}=\mathrm{f}_{\mathrm{cw}} \cdot \rho_{\mathrm{cw}} \cdot \frac{\mathrm{v}_{\mathrm{cw}}^{2}}{2} \cdot \frac{\mathrm{L}_{\text {tube,total }}}{\mathrm{d}_{\text {tube, } \mathrm{i}}}
$$

The tube conductance $\left(\mathrm{UA}_{\text {tube }}\right)$ is related to conduction through the tubes and is calculated using the thermal conductivity of the tubing.

$$
\mathrm{UA}_{\text {tube }}=\frac{2 \cdot \pi \cdot \mathrm{k}_{\text {tube }} \cdot \mathrm{L}_{\mathrm{HX}} \cdot \mathrm{N}_{\text {tubes, Xsection }}}{\ln \left(\frac{\mathrm{d}_{\text {tube, } \mathrm{o}}}{\mathrm{d}_{\text {tube, } \mathrm{i}}}\right)}
$$

The shell-side heat transfer coefficient correlation requires the tube surface temperature. The tube surface temperature ( $\left.\mathrm{T}_{\text {tube,surface }}\right)$ is approximated using the average condenser water temperature.

$$
\mathrm{T}_{\text {tube,surface }}=\mathrm{T}_{\mathrm{cw}}
$$


The refrigerant side heat transfer coefficient ( $h \mathrm{ht}_{\mathrm{ref}}$ ) is calculated using a correlation applicable to falling film condensation over a bank of horizontal tubes. (Incropera and DeWitt, 2002).

$$
\mathrm{htc}_{\text {ref }}=0.729 \cdot\left(\frac{\operatorname{abs}\left(\mathrm{g} \cdot \rho_{1, \text { ref }} \cdot\left(\rho_{1, \text { ref }}-\rho_{\mathrm{v}, \text { ref }}\right) \cdot \mathrm{k}_{1, \text { ref }}^{3} \cdot \mathrm{h}_{\mathrm{fg}, \text { ref }}\right)}{\operatorname{abs}\left(\mu_{1, \text { ref }} \cdot\left(\mathrm{T}_{1}-\mathrm{T}_{\text {tube, surface }}\right) \cdot \mathrm{d}_{\text {tube, } \mathrm{o}}\right)}\right)^{1 / 4}
$$

where $g$ is the acceleration due to gravity.

The conductance on the refrigerant side is calculated using the heat transfer coefficient and the effective heat transfer area.

$$
\mathrm{UA}_{\text {ref }}=\mathrm{htc}_{\text {ref }} \cdot \mathrm{N}_{\text {tubes }} \cdot \pi \cdot \mathrm{d}_{\text {tube, } \mathrm{o}} \cdot \mathrm{L}_{\text {tube, total }}
$$

The refrigerant side pressure drop is a function of the maximum refrigerant velocity $\left(\mathrm{v}_{\text {max,ref }}\right)$, which is calculated using conservation of mass applied at the shell midplane.

$$
\mathrm{v}_{\text {max }, \text { ref }}=\frac{\dot{\mathrm{m}}_{\text {ref }}}{\rho_{\mathrm{v}, \text { ref }} \cdot \mathrm{L}_{\mathrm{HX}} \cdot\left(\frac{\mathrm{D}_{\mathrm{HX}}-\mathrm{N}_{\text {tubes, vert }} \cdot \mathrm{d}_{\text {tube, }}}{\mathrm{N}_{\text {baffles }}}\right)}
$$

The maximum refrigerant Reynolds number $\left(\mathrm{Re}_{\mathrm{max}, \mathrm{ref}}\right)$ is calculated based on the maximum refrigerant velocity.

$$
\operatorname{Re}_{\text {max,ref }}=\frac{\rho_{\mathrm{v}, \text { ref }} \cdot \mathrm{v}_{\text {max,ref }} \cdot \mathrm{d}_{\text {tube,o }}}{\mu_{\mathrm{v}, \text { ref }}}
$$

The friction factor for a tube bank ( $f_{\text {tube,bank }}$ ) is assumed to be constant based on the calculated Reynolds number and the correlation shown in Incropera and DeWitt (2002).

$$
\mathrm{f}_{\text {tube,bank }}=0.6
$$

The correction factor for a tube bank $\left(\chi_{\text {tube, bank }}\right)$ based on the packing pattern is: 


$$
\chi_{\text {tube,bank }}=1.25
$$

The refrigerant side pressure drop over the tube bank is calculated using the following correlation for flow through a tube bank, found in Incropera and DeWitt (2002).

$$
\Delta \mathrm{p}_{\text {ref }}=\mathrm{N}_{\text {tubes, vert }} \cdot \mathrm{N}_{\text {baffles }} \cdot \chi_{\text {tube, bank }} \cdot \mathrm{f}_{\text {tube, bank }} \cdot \rho_{\mathrm{v}, \text { ref }} \cdot \frac{\mathrm{v}_{\text {max,ref }}^{2}}{2}
$$

The total conductance of the heat exchanger is related to the sum of the conductances of the tube side, the actual tubes, and the shell side. These are equated in Equation (4.106). Multipliers accounting for surface enhancements are set such that the condenser size matches closely with industry average, as supplied by ARI (2004).

$$
\mathrm{UA}_{\text {total }}=\left(\left(2 \cdot \mathrm{UA}_{\mathrm{cw}}\right)^{-1}+\mathrm{UA}_{\text {tube }}^{-1}+\left(9.5 \cdot \mathrm{UA}_{\text {ref }}\right)^{-1}\right)^{-1}
$$

The required conductance calculated using the $\varepsilon-\mathrm{NTU}$ method is equated to the total conductance calculated above.

$$
\mathrm{UA}_{\text {req }}=\mathrm{UA}_{\text {total }}
$$

In a similar manner, the model equates the required tube-side pressure drop, a design parameter for the condenser water side, to the pressure drop calculated in the pressure drop equations.

$$
\frac{\Delta \mathrm{p}_{\mathrm{cw}}-\Delta \mathrm{p}_{\mathrm{cw}, \mathrm{req}}}{\Delta \mathrm{p}_{\mathrm{cw}, \text { req }}}=0
$$

The heat exchanger model varies the geometry (shell length and diameter) until the specified performance targets are met using a non-linear, root finding technique. 


\subsubsection{Size}

The design parameters that were used in the calculation of the size of an indirectcontact condenser (Table 9) using R-134a as a refrigerant are listed in Table 8. Further optimization of this heat exchanger is described in Chapter 5.

Table 9 Indirect-contact condenser dimensions based on ARTI standard conditions.

\begin{tabular}{l|l|l} 
Dimension & Symbol & Value \\
\hline Heat exchanger length & $\mathrm{L}_{\mathrm{HX}}$ & $13 \mathrm{ft}$ \\
Heat exchanger diameter & $\mathrm{D}_{\mathrm{HX}}$ & $36 \mathrm{in}$ \\
Number of tubes & $\mathrm{N}_{\text {tubes }}$ & 910 \\
Tube pitch & Pitch & 0.975 in
\end{tabular}

The ability of the model to approximately predict the size of a falling film condenser consistent with those found in today's 1000-ton chillers is some measure of its validation.

\subsubsection{Cost}

Cost data supplied by the manufacturers is used to estimate that the total cost for a shell-and-tube condenser sized for a 1000-ton R-134a chiller. The estimated cost is $\$ 18,300$. The material cost is assumed to scale with size and is divided roughly into two parts: the shell cost and the tube costs. The shell cost is assumed to be a function of the volume enclosed by the shell $\left(\mathrm{V}_{\text {shell }}\right)$ in cubic feet. The total tube cost is assumed to be a function of the tube length. The fixed costs associated with fabricating shell-and-tube condensers on a mass scale are approximately $60 \%$ of the sum of the shell and tube costs.

$$
\begin{gathered}
\text { Shell Cost }=\left(\frac{\$ 25.50}{\mathrm{ft}^{3}}\right) \cdot \frac{\pi}{4} \cdot \mathrm{D}_{\mathrm{HX}, \mathrm{ft}}^{2} \cdot \mathrm{L}_{\mathrm{HX}, \mathrm{ft}} \\
\text { Tube Cost }=\left(\frac{\$ 0.76}{\mathrm{ft}}\right) \cdot \mathrm{N}_{\text {tubes }} \cdot \mathrm{L}_{\mathrm{HX}, \mathrm{ft}}
\end{gathered}
$$




\subsubsection{Indirect-contact Evaporator Model}

An indirect-contact evaporator for a typical R-134a chiller is similar to the condenser: the major difference being that more care is taken to minimize the approach temperature. The design, performance, and cost of an indirect-contact evaporator for a 1000-ton R-134a chiller are described in the following sections. To account for surface enhancements, the governing equations for determining the heat transfer coefficients in the indirect-contact evaporator model are calibrated such that the size and cost are approximately equivalent to an industry-average (ARI 2004).

\subsubsection{Performance}

This section is a description of an indirect-contact evaporator model constructed in the EES environment. Table 10 shows the user-specified design parameters used to predict the performance, size and cost of an evaporator.

Table 10 Indirect-contact evaporator design parameters.

\begin{tabular}{l|c|c} 
Parameter & Symbol & Default value \\
\hline Approach temperature & $\Delta \mathrm{T}_{\text {evap }}$ & varied \\
Chilled water velocity & $\mathrm{v}_{\text {chw }}$ & $3 \mathrm{ft} / \mathrm{s}$ \\
Tubing material & $\mathrm{T} \$$ & 'Copper' \\
Tube pitch-to-tube OD ratio & Pitch $\$ tubeOD & 1.4 \\
Heat exchanger aspect ratio & $\mathrm{AR}(\mathrm{L} / \mathrm{D})$ & 5 \\
\hline Refrigerant mass flow rate & $\dot{\mathrm{m}}_{\text {ref }}$ & $52.3 \mathrm{lbm} / \mathrm{s}$ \\
Refrigerant inlet enthalpy & $\mathrm{h}_{1}$ & $45.12 \mathrm{Btu} / \mathrm{lbm}$ \\
Chilled water inlet temperature & $\mathrm{T}_{3}$ & $54^{\circ} \mathrm{F}$ \\
Chilled water exit temperature & $\mathrm{T}_{4}$ & $44^{\circ} \mathrm{F}$
\end{tabular}

Figure 36 is a schematic of a shell and tube evaporator, the refrigerant is assumed to be on the shell side and transferring heat in the pool boiling regime for a flooded evaporator. 


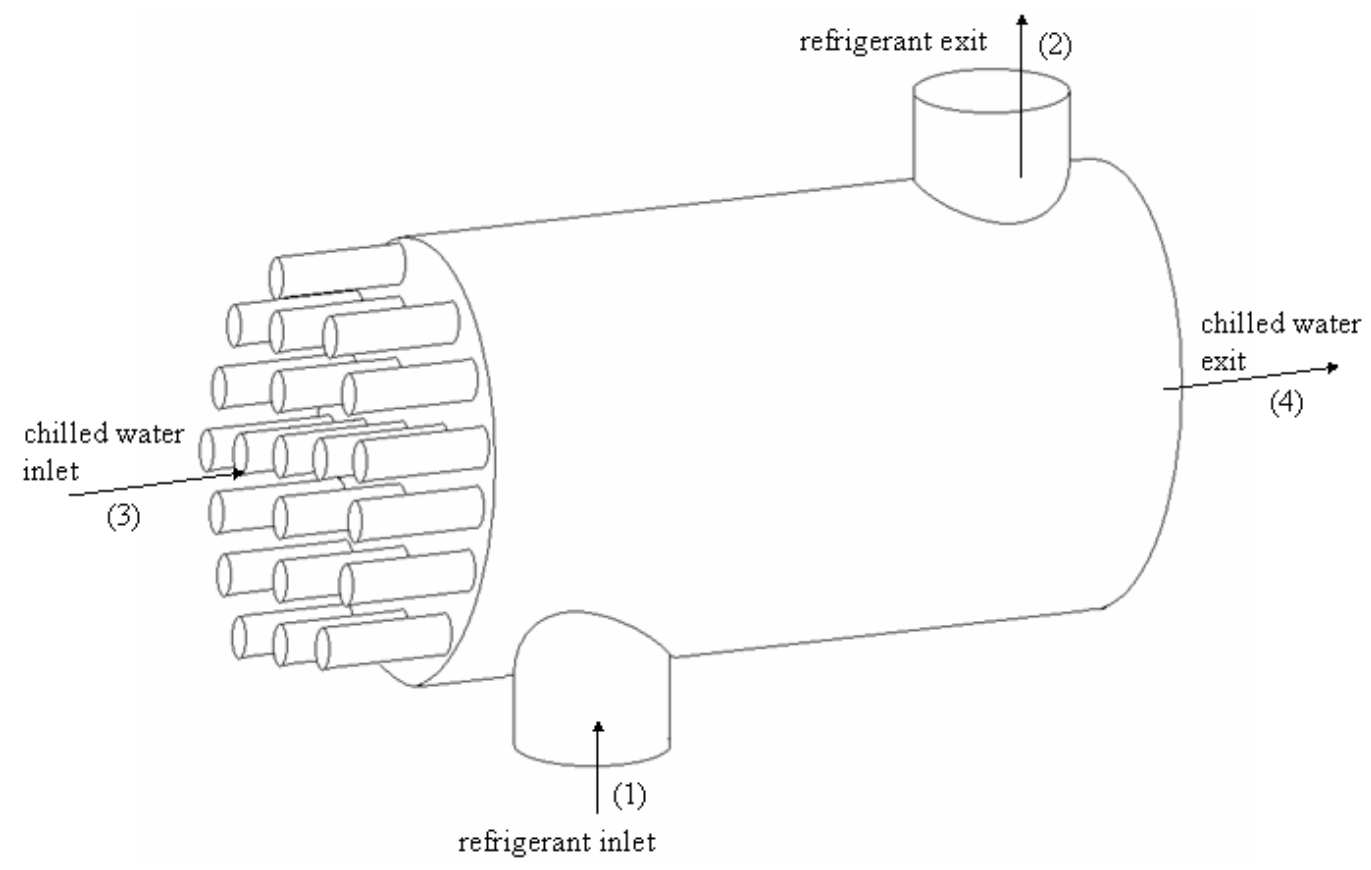

Figure 36 Schematic of an indirect-contact evaporator.

The chilled water flows through the tubes and rejects heat to the evaporating refrigerant. The chilled water temperature drops by $10^{\circ} \mathrm{F}$ passing through the tubes. The remainder of this section is devoted to the determination of the size and performance of an indirect-contact evaporator.

Analysis of any real heat exchangers shows that increasing size yields better heat exchanger performance, but not without penalty. Increasing heat exchanger size (effective heat transfer area) an incremental amount follows the law of diminishing returns. With penalties in pressure drop, in driving temperature gradient, and in cost, the size of a heat exchanger can be optimized for a particular application. The maximum allowable pressure drops, required approach temperatures, and the length of service, among other factors can dictate the optimal heat exchanger size. One goal of this project is to determine the trade-offs for varying component sizes en route to finding an optimal configuration. With a high pressure refrigerant like R-134a, large absolute pressure drops 
are not as significant as with low pressure refrigerants like R-123 and water. The pressure drop, relative to the refrigerant absolute pressure, is a parameter critical in the cycle performance. For the evaporator model, the pressure drop for the refrigerant side is determined based on the tube spacing (pitch). On the water side, the pressure drop is determined based on the water velocity and the fluid friction through the tubing.

Immediately prior to entering the evaporator, the refrigerant is throttled through an expansion device (usually an orifice plate in large chillers), which is assumed to be isenthalpic. This allows the condenser exit enthalpy to be set equal to the enthalpy of the evaporator inlet.

$$
\mathrm{h}_{1}=\mathrm{h}_{\text {cond,out }}
$$

The refrigerant inlet temperature $\left(T_{1}\right)$ is determined by the approach temperature difference $\left(\Delta \mathrm{T}_{\text {evap }}\right)$, which is a design parameter.

$$
\mathrm{T}_{1}=\mathrm{T}_{4}-\Delta \mathrm{T}_{\text {evap }}
$$

The remainder of the state properties for the refrigerant side evaporator inlet is calculated using the enthalpy and temperature.

$$
\begin{array}{r}
\mathrm{x}_{1}=\operatorname{quality}\left(\mathrm{R} 134 \mathrm{a}, \mathrm{T}=\mathrm{T}_{1}, \mathrm{~h}=\mathrm{h}_{1}\right) \\
\mathrm{p}_{1}=\operatorname{\operatorname {pressure}}\left(\mathrm{R} 134 \mathrm{a}, \mathrm{T}=\mathrm{T}_{1}, \mathrm{~h}=\mathrm{h}_{1}\right) \\
\mathrm{s}_{1}=\operatorname{entropy}\left(\mathrm{R} 134 \mathrm{a}, \mathrm{T}=\mathrm{T}_{1}, \mathrm{~h}=\mathrm{h}_{1}\right) \\
\mathrm{v}_{1}=\operatorname{volume}\left(\mathrm{R} 134 \mathrm{a}, \mathrm{T}=\mathrm{T}_{1}, \mathrm{~h}=\mathrm{h}_{1}\right)
\end{array}
$$

The refrigerant exit pressure $\left(\mathrm{p}_{2}\right)$ is related to the refrigerant pressure drop $\left(\Delta \mathrm{p}_{\mathrm{ref}}\right)$ which will ultimately be related to the heat exchanger geometry.

$$
\mathrm{p}_{2}=\mathrm{p}_{1}-\Delta \mathrm{p}_{\text {ref }}
$$


For R-134a as a refrigerant, the refrigerant side pressure drop is small relative to the absolute pressure. The refrigerant exit properties are calculated using the assumption that the R-134a is a saturated vapor at the evaporator exit.

$$
\mathrm{x}_{2}=1
$$

The remainder of the state properties at the refrigerant exit is calculated using the pressure and quality.

$$
\begin{array}{r}
\mathrm{T}_{2}=\text { temperature }\left(\mathrm{R} 134 \mathrm{a}, \mathrm{p}=\mathrm{p}_{2}, \mathrm{x}=\mathrm{x}_{2}\right) \\
\mathrm{h}_{2}=\operatorname{enthalpy}\left(\mathrm{R} 134 \mathrm{a}, \mathrm{p}=\mathrm{p}_{2}, \mathrm{x}=\mathrm{x}_{2}\right) \\
\mathrm{s}_{2}=\operatorname{entropy}\left(\mathrm{R} 134 \mathrm{a}, \mathrm{p}=\mathrm{p}_{2}, \mathrm{x}=\mathrm{x}_{2}\right) \\
\mathrm{v}_{2}=\operatorname{volume}\left(\mathrm{R} 134 \mathrm{a}, \mathrm{p}=\mathrm{p}_{2}, \mathrm{x}=\mathrm{x}_{2}\right)
\end{array}
$$

The model calculates the chilled water inlet and exit conditions using the ARTI specified temperatures and the water side pressure drop.

$$
\mathrm{p}_{3}=\left(1+\frac{\mathrm{p}_{\text {drop, chw }}}{100}\right) \cdot \mathrm{p}_{\text {atm }}
$$

The remainder of the state properties for the chilled water inlet is determined using the temperature and the pressure.

$$
\begin{array}{r}
\mathrm{h}_{3}=\operatorname{enthalpy}\left(\mathrm{R} 134 \mathrm{a}, \mathrm{T}=\mathrm{T}_{3}, \mathrm{p}=\mathrm{p}_{3}\right) \\
\mathrm{s}_{3}=\operatorname{entropy}\left(\mathrm{R} 134 \mathrm{a}, \mathrm{T}=\mathrm{T}_{3}, \mathrm{p}=\mathrm{p}_{3}\right) \\
\mathrm{v}_{3}=\operatorname{volume}\left(\mathrm{R} 134 \mathrm{a}, \mathrm{T}=\mathrm{T}_{3}, \mathrm{p}=\mathrm{p}_{3}\right) \\
\mathrm{x}_{3}=\operatorname{quality}\left(\mathrm{R} 134 \mathrm{a}, \mathrm{T}=\mathrm{T}_{3}, \mathrm{p}=\mathrm{p}_{3}\right)
\end{array}
$$

The chilled water exit temperature $\left(\mathrm{T}_{4}\right)$ is:

$$
\mathrm{T}_{4}=\mathrm{T}_{\text {chillwater,out }}
$$

The chilled water exit is at atmospheric pressure.

$$
\mathrm{p}_{4}=\mathrm{p}_{\mathrm{atm}}
$$


The remainder of the chilled water exit state properties is determined in the following equations.

$$
\begin{array}{r}
\mathrm{h}_{4}=\operatorname{enthalpy}\left(\mathrm{R} 134 \mathrm{a}, \mathrm{T}=\mathrm{T}_{4}, \mathrm{p}=\mathrm{p}_{4}\right) \\
\mathrm{s}_{4}=\operatorname{entropy}\left(\mathrm{R} 134 \mathrm{a}, \mathrm{T}=\mathrm{T}_{4}, \mathrm{p}=\mathrm{p}_{4}\right) \\
\mathrm{v}_{4}=\operatorname{volume}\left(\mathrm{R} 134 \mathrm{a}, \mathrm{T}=\mathrm{T}_{4}, \mathrm{p}=\mathrm{p}_{4}\right) \\
\mathrm{x}_{4}=\operatorname{quality}\left(\mathrm{R} 134 \mathrm{a}, \mathrm{T}=\mathrm{T}_{4}, \mathrm{p}=\mathrm{p}_{4}\right)
\end{array}
$$

The evaporator model can calculate the size and geometry of the evaporator corresponding to the end states just determined. The chilled water properties are required to determine the water side heat transfer coefficient and pressure drop and are based on the average pressure $\left(\mathrm{p}_{\text {chw }}\right)$ and temperature $\left(\mathrm{T}_{\text {chw }}\right)$.

$$
\begin{aligned}
& \mathrm{p}_{\text {chw }}=\frac{\mathrm{p}_{3}+\mathrm{p}_{4}}{2} \\
& \mathrm{~T}_{\text {chw }}=\frac{\mathrm{T}_{3}+\mathrm{T}_{4}}{2}
\end{aligned}
$$

The following properties are calculated for the chilled water: the constant pressure specific heat $\left(\mathrm{C}_{\mathrm{p}, \mathrm{chw}}\right)$, density $\left(\rho_{\text {chw }}\right)$, viscosity $\left(\mu_{\text {chw }}\right)$, thermal conductivity $\left(\mathrm{k}_{\mathrm{chw}}\right)$, and Prandtl number $\left(\operatorname{Pr}_{\text {chw }}\right)$.

$$
\begin{array}{r}
\mathrm{C}_{\mathrm{p}, \text { chw }}=\mathrm{cP}\left(\text { 'Water', } \mathrm{p}=\mathrm{p}_{\text {chw }}, \mathrm{T}=\mathrm{T}_{\text {chw }}\right) \\
\rho_{\text {chw }}=\operatorname{density}\left(\text { 'Water, } \mathrm{p}=\mathrm{p}_{\text {chw }}, \mathrm{T}=\mathrm{T}_{\text {chw }}\right) \\
\mu_{\text {chw }}=\text { viscosity('Water', } \mathrm{p}=\mathrm{p}_{\text {chw }}, \mathrm{T}=\mathrm{T}_{\text {chw }} \text { ) } \\
\mathrm{k}_{\text {chw }}=\text { conductivity('Water', } \mathrm{p}=\mathrm{p}_{\text {chw }}, \mathrm{T}=\mathrm{T}_{\text {chw }} \text { ) } \\
\left.\operatorname{Pr}_{\text {chw }}=\operatorname{prandtl('Water',~} \mathrm{p}=\mathrm{p}_{\text {chw }}, \mathrm{T}=\mathrm{T}_{\text {chw }}\right)
\end{array}
$$

The tube inner diameter $\left(\mathrm{d}_{\text {tube, }}\right)$ and thickness ( $\left.\mathrm{t}_{\text {tube }}\right)$ is assumed to be consistent with a standard 3/4" OD refrigeration tube (McMaster-Carr, 2003).

$$
\begin{aligned}
& \mathrm{d}_{\text {tube, } \mathrm{i}}=0.70[\mathrm{in}] \\
& \mathrm{t}_{\text {tube }}=0.025[\mathrm{in}]
\end{aligned}
$$


The tube outer diameter is calculated using the tube thickness and the inner diameter.

$$
\mathrm{d}_{\text {tube, } \mathrm{o}}=\mathrm{d}_{\text {tube, } \mathrm{i}}+2 \cdot \mathrm{t}_{\text {tube }}
$$

The separation between tube centers (pitch) is determined by a design parameter that is a ratio of the tube pitch to the tube outer diameter (pitch $\backslash$ tubeOD).

$$
\text { pitch }=\text { pitch } \backslash \text { tubeOD } \cdot \mathrm{d}_{\text {tube,o }}
$$

The cross-sectional area of the shell is determined in terms of the diameter, assuming a cylindrical geometry.

$$
\mathrm{A}_{\mathrm{HX}}=\frac{\pi}{4} \cdot \mathrm{D}_{\mathrm{HX}}^{2}
$$

The evaporator aspect ratio (AR), a design parameter, is used to calculate the ratio between the shell length and the diameter.

$$
\mathrm{AR}=\frac{\mathrm{L}_{\mathrm{HX}}}{\mathrm{D}_{\mathrm{HX}}}
$$

The maximum number of tubes that can be vertically stacked including the tube pitch on top of each other and still fit inside the shell $\left(\mathrm{N}_{\text {tubes,vert }}\right)$ is calculated using the shell diameter and the tube pitch.

$$
\mathrm{N}_{\text {tubes, vert }}=\frac{\mathrm{D}_{\mathrm{HX}}}{\text { pitch }}
$$

The number of tubes that will fit in the shell cross section is found using a geometric relationship that relates the area to the tube array pattern. The tube array is assumed to be hexagonal closed packed.

$$
\mathrm{N}_{\text {tubes }, \text { Xsection }}=\frac{\mathrm{A}_{\mathrm{HX}}}{2 \cdot \mathrm{pitch}^{2} \cdot \sin \left(\frac{\pi}{3}\right) \cdot \cos \left(\frac{\pi}{3}\right)}
$$


The number of tubes in the shell cross section dictates the number of tube-passes that are required to provide adequate heat transfer area. The number of tube-passes $\left(\mathrm{N}_{\text {pass }}\right)$ is determined.

$$
\mathrm{N}_{\text {pass }}=\frac{\mathrm{N}_{\text {tubes, Xsection }}}{\mathrm{N}_{\text {tubes }}}
$$

The total length of the tubes $\left(\mathrm{L}_{\text {tube,total }}\right)$ is calculated using the number of tube passes and the shell length in the following equation.

$$
\mathrm{L}_{\text {tube,total }}=\mathrm{N}_{\text {pass }} \cdot \mathrm{L}_{\mathrm{HX}}
$$

In order to determine the actual tube conductance $\left(\mathrm{UA}_{\text {tube }}\right)$, the thermal conductivity of the tube is calculated using EES built in thermophysical property functions for solids.

$$
\mathrm{k}_{\text {tube }}=\mathrm{k}_{-}\left(\text {Copper, } \frac{\mathrm{T}_{3}+\mathrm{T}_{4}}{2}\right)
$$

The evaporator model calculates the refrigerant side properties required to determine the shell side conductance. These properties are calculated for saturated liquid and vapor at the evaporator exit temperature. The density of saturated liquid $\left(\rho_{1, \text { ref }}\right)$ and saturated vapor $\left(\rho_{\mathrm{v}, \text { ref }}\right)$, thermal conductivity of saturated liquid $\left(\mathrm{k}_{\mathrm{v}, \mathrm{ref}}\right)$ and saturated vapor $\left(\mathrm{k}_{1, \text { ref }}\right)$, the enthalpy of vaporization $\left(\mathrm{h}_{\mathrm{fg}, \mathrm{ref}}\right)$, the viscosity of saturated liquid $\left(\mu_{1, \text { ref }}\right)$ and saturated vapor $\left(\mu_{\mathrm{v}, \mathrm{ref}}\right)$, the constant pressure specific heat of the vapor $\left(\mathrm{C}_{\mathrm{p}, \mathrm{v}, \mathrm{ref}}\right)$, the kinematic viscosity of the vapor $\left(v_{v, \text { ref }}\right)$, and the Prandtl number $\left(\mathrm{Pr}_{\mathrm{ref}}\right)$ all of R-134a evaluated at the exit temperature are calculated. 


$$
\begin{array}{r}
\rho_{1, \text { ref }}=\operatorname{density}\left(\mathrm{R} 134 \mathrm{a}, \mathrm{T}=\mathrm{T}_{2}, \mathrm{x}=0\right) \\
\rho_{\mathrm{v}, \text { ref }}=\operatorname{density}\left(\mathrm{R} 134 \mathrm{a}, \mathrm{T}=\mathrm{T}_{2}, \mathrm{x}=1\right) \\
\mathrm{k}_{1, \text { ref }}=\operatorname{conductivity}\left(\mathrm{R} 134 \mathrm{a}, \mathrm{T}=\mathrm{T}_{2}, \mathrm{x}=0\right) \\
\mathrm{k}_{\mathrm{v}, \mathrm{ref}}=\operatorname{conductivity}\left(\mathrm{R} 134 \mathrm{a}, \mathrm{T}=\mathrm{T}_{2}, \mathrm{x}=1\right) \\
\mathrm{hfg}_{\mathrm{ref}}=\mathrm{h}_{2}-\operatorname{enthalpy}\left(\mathrm{R} 134 \mathrm{a}, \mathrm{T}=\mathrm{T}_{2}, \mathrm{x}=0\right) \\
\mu_{1, \text { ref }}=\operatorname{viscosity}\left(\mathrm{R} 134 \mathrm{a}, \mathrm{T}=\mathrm{T}_{2}, \mathrm{x}=0\right) \\
\mu_{\mathrm{v}, \mathrm{ref}}=\operatorname{viscosity}\left(\mathrm{R} 134 \mathrm{a}, \mathrm{T}=\mathrm{T}_{2}, \mathrm{x}=1\right) \\
\mathrm{C}_{\mathrm{p}, \mathrm{v}}=\mathrm{cP}\left(\mathrm{R} 134 \mathrm{a}, \mathrm{T}=\mathrm{T}_{2}, \mathrm{x}=1\right) \\
\operatorname{Pr}_{\text {ref }}=\operatorname{Prandt}\left(\mathrm{R} 134 \mathrm{a}, \mathrm{T}=\mathrm{T}_{2}, \mathrm{x}=1\right) \\
v_{\mathrm{v}, \mathrm{ref}}=\frac{\mu_{\mathrm{v}, \mathrm{ref}}}{\rho_{\mathrm{v}, \mathrm{ref}}}
\end{array}
$$

The evaporator model calculates the required heat transfer $\left(\dot{\mathrm{Q}}_{\text {evap }}\right)$ and the required chilled water mass flow rate using energy balances.

$$
\begin{gathered}
\dot{\mathrm{Q}}_{\text {evap }}=\dot{\mathrm{m}}_{\text {ref }} \cdot\left(\mathrm{h}_{2}-\mathrm{h}_{1}\right) \\
\dot{\mathrm{m}}_{\text {chw }}=\frac{\dot{\mathrm{Q}}_{\text {evap }}}{\mathrm{C}_{\mathrm{p}, \mathrm{chw}} \cdot\left(\mathrm{T}_{3}-\mathrm{T}_{4}\right)}
\end{gathered}
$$

The total required conductance is calculated using effectiveness-NTU relationships $(\varepsilon-\mathrm{NTU})$. The effectiveness ( $\left.\varepsilon_{\text {evap }}\right)$ is defined by:

$$
\varepsilon_{\text {evap }}=\frac{\mathrm{T}_{3}-\mathrm{T}_{4}}{\mathrm{~T}_{3}-\mathrm{T}_{2}}
$$

The number of transfer units (NTU) is defined in the following relationship, based on the assumption that the capacitance ratio is equal to zero (i.e. $\mathrm{C}_{\mathrm{r}}=\frac{\dot{\mathrm{C}}_{\min }}{\dot{\mathrm{C}}_{\max }}=0$ ).

$$
\mathrm{NTU}=-\ln \left(1-\varepsilon_{\text {evap }}\right)
$$

The required overall heat exchanger conductance is calculated using the definition of NTU. 


$$
\mathrm{UA}_{\text {req }}=\mathrm{NTU} \cdot \dot{\mathrm{m}}_{\mathrm{chw}} \cdot \mathrm{C}_{\mathrm{p}, \mathrm{chw}}
$$

The water side heat transfer coefficient is determined in the following group of equations. The chilled water velocity is also used to determine the pressure drop through the piping. It is calculated using a Nusselt number correlation that depends on the chilled water velocity $\left(\mathrm{v}_{\mathrm{chw}}\right)$, Reynolds number $\left(\mathrm{Re}_{\mathrm{chw}}\right)$, and friction factor $\left(\mathrm{f}_{\mathrm{chw}}\right)$. The friction factor is calculated using a correlation for turbulent flow through smooth tubes (Incropera and DeWitt, 2002). The Reynolds number and friction factor are calculated.

$$
\begin{gathered}
\operatorname{Re}_{\text {chw }}=\frac{\rho_{\text {chw }} \cdot \mathrm{v}_{\text {chw }} \cdot \mathrm{d}_{\text {tube }, \mathrm{i}}}{\mu_{\text {chw }}} \\
\mathrm{f}_{\text {chw }}=\left(0.79 \cdot \ln \left(\operatorname{Re}_{\text {chw }}\right)-1.64\right)^{-2}
\end{gathered}
$$

The chilled water velocity is set to $3 \mathrm{ft} / \mathrm{s}$ and therefore the number of tubes is set by:

$$
\mathrm{v}_{\text {chw }}=\frac{\dot{\mathrm{m}}_{\mathrm{chw}}}{\rho_{\text {chw }} \cdot \mathrm{N}_{\text {tubes }} \cdot \frac{\pi}{4} \cdot \mathrm{d}_{\text {tube }, \mathrm{i}}^{2}}
$$

The pressure drop is specified as a percentage of its absolute pressure $\left(\Delta \mathrm{p}_{\text {chw,req }}\right)$.

$$
\Delta \mathrm{p}_{\text {chw }, \text { req }}=\left(\frac{\mathrm{p}_{\text {drop,chw }}}{100}\right) \cdot \mathrm{p}_{4}
$$

The Nusselt number is calculated by: (Incropera and DeWitt, 2002).

$$
\operatorname{Nus}_{\text {chw }}=\frac{\left(\frac{f_{\text {chw }}}{8}\right) \cdot \operatorname{Re}_{\text {chw }} \cdot \operatorname{Pr}_{\text {chw }}}{1.07+12.7 \cdot\left(\frac{f_{\text {chw }}}{8}\right)^{1 / 2} \cdot\left(\operatorname{Pr}_{\text {chw }}^{2 / 3}-1\right)}
$$

Using the definition of the Nusselt number, the heat transfer coefficient for the water side is calculated. 


$$
h t c_{\text {chw }}=\frac{\mathrm{Nus}_{\text {chw }} \cdot \mathrm{k}_{\mathrm{chw}}}{\mathrm{d}_{\text {tube, } \mathrm{i}}}
$$

Thus the water side conductance $\left(\mathrm{UA}_{\mathrm{chw}}\right)$ is calculated using the definition of conductance.

$$
\mathrm{UA}_{\text {chw }}=\mathrm{htc}_{\text {chw }} \cdot \mathrm{N}_{\text {tube, } \mathrm{Xsection}} \cdot \pi \cdot \mathrm{d}_{\text {tube, } \mathrm{i}} \cdot \mathrm{L}_{\mathrm{HX}}
$$

The pressure drop is calculated according to:

$$
\Delta \mathrm{p}_{\text {chw }}=\mathrm{f}_{\text {chw }} \cdot \rho_{\text {chw }} \cdot \frac{\mathrm{v}_{\text {chw }}^{2}}{2} \cdot\left(\frac{\mathrm{L}_{\text {tube,total }}}{\mathrm{d}_{\text {tube }, \mathrm{i}}}\right)
$$

The tube conductance $\left(\mathrm{UA}_{\text {tube }}\right)$ is calculated.

$$
\mathrm{UA}_{\text {tube }}=\frac{2 \cdot \pi \cdot \mathrm{k}_{\text {tube }} \cdot \mathrm{L}_{\mathrm{HX}} \cdot \mathrm{N}_{\text {tubes }, \mathrm{X} \text { section }}}{\ln \left(\frac{\mathrm{d}_{\text {tube }, \mathrm{o}}}{\mathrm{d}_{\text {tube }, \mathrm{i}}}\right)}
$$

The following equations calculate the shell side heat transfer coefficient and the pressure drop. The characteristic driving temperature gradient $\left(\Delta \mathrm{T}_{\mathrm{e}}\right)$ is approximated by:

$$
\Delta \mathrm{T}_{\mathrm{e}}=\mathrm{T}_{\text {tube,surface }}-\mathrm{T}_{1}
$$

where $T_{\text {tube,surface }}$ is the average temperature of the chilled water.

The heat transfer coefficient is determined using a Nusselt number correlation. The amount of superheat required to promote nucleation sites for boiling is calculated using a modified enthalpy of vaporization (Incropera and DeWitt, 2002).

$$
\mathrm{hfg}^{\prime}=\mathrm{hfg}_{\mathrm{ref}}+0.80 \cdot \mathrm{C}_{\mathrm{p}, \mathrm{v}} \cdot \Delta \mathrm{T}_{\mathrm{e}}
$$

A Nusselt number correlation appropriate for pool boiling around smooth circular cylinders is given by Incropera and DeWitt (2002). 


$$
\operatorname{Nus}_{\text {ref }}=\mathrm{C} \cdot\left(\frac{\mathrm{g} \cdot\left(\rho_{\mathrm{l}, \mathrm{ref}}-\rho_{\mathrm{v}, \mathrm{ref}}\right) \cdot \mathrm{hfg}^{\prime} \cdot \mathrm{d}_{\text {tube,o }}^{3}}{v_{\mathrm{v}, \text { ref }} \cdot \mathrm{k}_{\mathrm{v}, \text { ref }} \cdot \Delta \mathrm{T}_{\mathrm{e}}}\right)^{1 / 4}
$$

where $\mathrm{g}$ is the acceleration due to gravity and $\mathrm{C}$ is a constant that scales the Nusselt number based on the geometry. For horizontal cylinders, $\mathrm{C}$ is set to 0.62 (Incropera and DeWitt, 2002). Using the definition of the Nusselt number, the refrigerant side heat transfer coefficient is calculated.

$$
\mathrm{htc}_{\text {ref }}=\frac{\mathrm{Nus}_{\mathrm{ref}} \cdot \mathrm{k}_{\mathrm{l}, \mathrm{ref}}}{\mathrm{d}_{\text {tube,o }}}
$$

The refrigerant side conductance is calculated using the heat transfer coefficient and the effective heat transfer area.

$$
\mathrm{UA}_{\text {ref }}=\mathrm{htc}_{\text {ref }} \cdot \mathrm{N}_{\text {pass }} \cdot \mathrm{N}_{\text {tubes }} \cdot \pi \cdot \mathrm{d}_{\text {tube, } \mathrm{o}} \cdot \mathrm{L}_{\mathrm{HX}}
$$

The pressure drop is calculated for flow of a liquid around a bank of tube using the same correlation used in the condenser model. The pressure loss is found to be very small $(<<1$ psi). For this reason, the refrigerant inlet and exit pressures are considered equal.

The individual conductances are combined in the following equation. The overall conductance is adjusted to account for enhanced surfaces. Multipliers are set such that the evaporator size matches closely with industry average, as suggested by supplied data (ARI 2004).

$$
\mathrm{UA}_{\text {total }}=\left(\left(2 \cdot \mathrm{UA}_{\mathrm{chw}}\right)^{-1}+\mathrm{UA}_{\text {tube }}^{-1}+\left(3.5 \cdot \mathrm{UA}_{\mathrm{ref}}\right)^{-1}\right)^{-1}
$$

The calculated conductance is set equal to the required conductance resulting in a constraint on the heat exchanger geometry. 


$$
\frac{\mathrm{UA}_{\text {total }}-\mathrm{UA}_{\text {req }}}{\mathrm{UA}_{\text {req }}}=0
$$

The calculated water side pressure drop is set equal to the required pressure drop, providing a second constraint on the geometry.

$$
\frac{\Delta \mathrm{p}_{\text {chw }}-\Delta \mathrm{p}_{\text {chw,req }}}{\Delta \mathrm{p}_{\text {chw,req }}}=0
$$

\subsubsection{Size}

The design parameters that were used in the calculation of the size of an indirect evaporator model for R-134a as a refrigerant are listed in Table 11.

Table 11 Indirect-contact evaporator dimensions based on ARTI standard conditions for R-134a.

\begin{tabular}{l|l|l} 
Dimension & Symbol & Value \\
\hline Heat exchanger length & $\mathrm{L}_{\mathrm{HX}}$ & $14 \mathrm{ft}$ \\
Heat exchanger diameter & $\mathrm{D}_{\mathrm{HX}}$ & $42 \mathrm{in}$ \\
Number of tubes & $\mathrm{N}_{\text {tubes,Xsection }}$ & 690 \\
Tube pitch & Pitch & 1.05 in
\end{tabular}

\subsubsection{Cost}

Cost data supplied by ARI is used to estimate that the total cost for a shell-andtube evaporator sized for a 1000 -ton R-134a chiller will be $\$ 19,900$. The material cost is assumed to scale with size and is divided roughly into two parts: the shell cost and the tube costs. The shell cost is assumed to be a function of the volume enclosed by the shell $\left(\mathrm{V}_{\text {shell }}\right)$ in cubic feet. The total tube cost is assumed to be a function of the tube length. The fixed costs associated with fabricating shell-and-tube evaporators on a mass scale are approximately $60 \%$ of the sum of the shell and tube costs.

$$
\text { Shell Cost }=\left(\frac{\$ 22.20}{\mathrm{ft}^{3}}\right) \cdot \frac{\pi}{4} \cdot \mathrm{D}_{\mathrm{HX}, \mathrm{ft}}^{2} \cdot \mathrm{L}_{\mathrm{HX}, \mathrm{ft}}
$$




$$
\text { Tube Cost }=\left(\frac{\$ 0.91}{\mathrm{ft}}\right) \cdot \mathrm{N}_{\text {tubes }} \cdot \mathrm{L}_{\mathrm{HX}, \mathrm{ft}}
$$

\subsubsection{Pump Model}

Pumps are required to circulate a working fluid through a building in order to meet cooling loads and for circulating water to reject heat from the refrigeration system. The most common working fluid circulated in large building air-conditioning systems is water. For both cooling and heat rejection, a pump has to develop sufficient head to overcome fluid friction pressure losses through system components (heat exchangers, piping, control valves, spray nozzles, etc.) and any hydrostatic head imbalance for open hydronic systems (heat rejection systems). In addition to developing sufficient head, pumps must move the required volume flow rate of fluid to meet cooling loads and reject heat. Pump selection requires that estimates of both flow rates and head be calculated.

The required flow rate is calculated using an energy balance through each heat exchanger. This balance is performed within the respective heat exchanger subprograms. The R-134a heat exchanger subprograms also have enough information to allow the pressure drops on the tube-side (water) to be estimated. The head loss through the evaporator will be a fraction of the total system head loss. The total system head loss is dependent on the design velocities for water piping, length of chilled water piping (supply and return), coil pressure drop, presence of fittings (elbows, tees), and control valves. For the purposes of this project, the total head loss for a chilled water piping system will be developed based on rules-of-thumb for a typical building. The first step is to estimate the total floor space capable of being conditioned with the nominal chiller 
capacity available. For commercial buildings, peak cooling loads are on the order of 400 $\mathrm{ft}^{2} /$ ton (ASHRAE, 1997).

$$
\text { Chilled Area }=400 \frac{\mathrm{ft}^{2}}{\text { ton }} \cdot \text { CoolCapacity }
$$

where 'CoolCapacity' is the chiller capacity in tons.

Thus, a 1000-ton chiller can be expected to meet the air-conditioning requirements of a building with an area of approximately $400,000 \mathrm{ft}^{2}$. For the piping loss calculation, the building is assumed to have 4-stories, each of which is $100,000 \mathrm{ft}^{2}$. The maximum pressure loss for the pipe is characterized by the greatest distance the water must travel through the building. For both the condenser water and chilled water piping systems, these lengths are illustrated schematically in Figure 37.

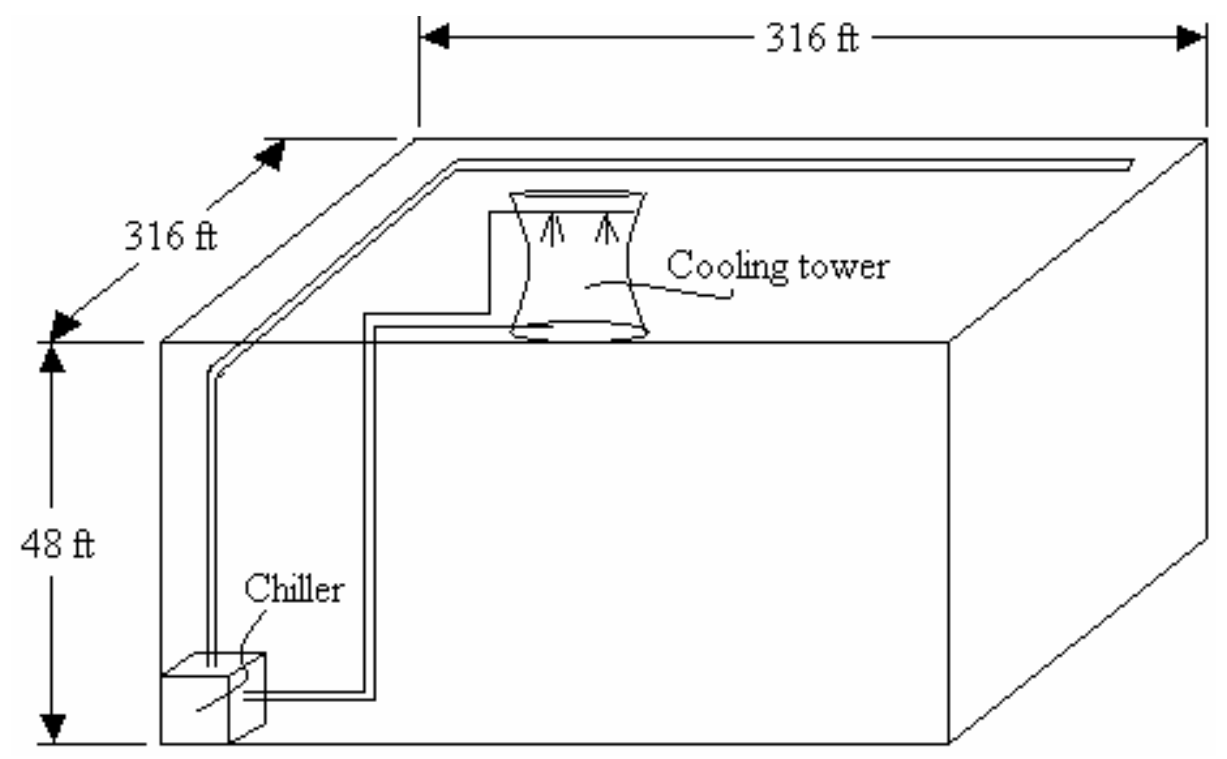

Figure 37 Illustration of piping configuration for a 4-story $400,000 \mathrm{ft}^{2}$ building.

A rule-of-thumb method used by Bell and Gossett (Bell and Gossett/ITT, 1998) to ball park estimate the pressure drop in piping for chilled water applications relevant to this study is as follows: 
1. estimate the longest run of pipe,

2. include an allowance of $50 \%$ for fittings to determine the effective pipe length, and

3. multiply the effective pipe length by a loss rate of 0.04 ( $4 \mathrm{ft}$ per $100 \mathrm{ft}$ of pipe) to obtain the required pump head (in $\mathrm{ft}$ of water) to overcome frictional losses

\section{Chilled Water Pump:}

The chilled water pump must overcome pressure losses in a relatively long piping loop and through the tubing of the evaporator. There are a number of parameters that the other chiller component models must calculate first in order to determine the overall pressure rise that the pump must supply. The pressure drop for the chilled water and the condenser water are supplied from the heat exchanger models. The evaporator pressure drop $\left(\Delta \mathrm{p}_{\text {evap }}\right)$ is calculated based on a number of factors described in Section 4.1.3.1.

$$
\Delta \mathrm{p}_{\text {evap }}=\Delta \mathrm{p}_{\mathrm{chw}}
$$

The maximum distance that the chilled water must travel is related to the piping pressure drop that the pump must overcome. The worst case pipe (i.e. longest run) traverses the building as shown in Figure 37. The overall dimensions of the building to be chilled are calculated using the assumptions that the building footprint $\left(\mathrm{A}_{\text {building }}\right)$ is a square and the height of each floor is $12 \mathrm{ft}$. For a 4-story building, the pipe is required to reach a height ( $\left.\mathrm{H}_{\text {building }}\right)$ of $48 \mathrm{ft}$. The pipe continues along the length and the width of the building, which are assumed equal. For a 100,000 sq ft footprint, the length and the width ( $\mathrm{L}_{\text {building }}$ ) are calculated using the following equation.

$$
\mathrm{L}_{\text {building }}=\sqrt{\mathrm{A}_{\text {building }}}
$$

Therefore, the length of pipe $\left(\mathrm{L}_{\text {pipe }}\right)$ expected in a building with a 1000-ton chiller is: 


$$
\mathrm{L}_{\text {pipe }}=2 \cdot\left(\mathrm{H}_{\text {building }}+2 \cdot \mathrm{L}_{\text {building }}\right)
$$

The maximum pipe length is used to obtain a rough estimate of what the effective length of the piping ( $\mathrm{L}_{\text {pipe,eff }}$ ) is when considering fittings, valves and other obstructions to flow. It is assumed these features effectively increase the pipe length by $50 \%$.

$$
\mathrm{L}_{\text {pipe,eff }}=1.5 \cdot \mathrm{L}_{\text {pipe }}
$$

This effective pipe length is used to determine the required pump head due to friction losses $\left(\mathrm{h}_{\text {pump }}\right)$ in the pipe. A loss rate of $4 \mathrm{ft}$ of head per $100 \mathrm{ft}$ of equivalent length of pipe is assumed to estimate the required head for the chilled water pump (head pump).

$$
\text { head }_{\text {pump }}=0.04 \cdot \mathrm{L}_{\text {pipe,eff }}
$$

The pressure rise that must be generated in order to overcome this frictional head loss is calculated.

$$
\Delta \mathrm{p}_{\text {pipe }}=\rho_{\text {chw }} \cdot \mathrm{g} \cdot \text { head }_{\text {pump }}
$$

where $\rho_{\text {chw }}$ is the density of chilled water and $\mathrm{g}$ is the acceleration due to gravity.

The total head that the pump must supply $\left(\Delta \mathrm{p}_{\text {pump }}\right)$ is the sum of the frictional pressure losses through the piping as well as the pressure loss through the evaporator ( $\Delta \mathrm{p}_{\text {evap }}$, supplied by the evaporator model).

$$
\Delta \mathrm{p}_{\text {pump }}=\Delta \mathrm{p}_{\text {pipe }}+\Delta \mathrm{p}_{\text {evap }}
$$

Based on this chilled water pump model, the total pressure required is $53 \mathrm{psi}$.

\section{Condenser Water Pump:}

The condenser water flow rate is calculated using an energy balance in the condenser. However, the condenser water loop differs from the chilled water loop in that it is assumed to be an open hydronic system; therefore, the pump must overcome an 
additional pressure loss associated with the hydrostatic head required to move water to the top of a cooling tower. Although the condenser water pump does not have to overcome pressure loss through cooling coils, it does have to overcome the pressure drop through spray nozzles located with the cooling tower. Thus, there are four pressure drop terms rather than two as in the chilled water pressure drop calculations. The condenser water does not need to be circulated throughout the building; it merely needs to be pumped up to the cooling tower making the frictional losses due to piping lower, depending on the location of the cooling tower.

The pressure drop through the condenser tubes is calculated in Section 4.1.3.1.

$$
\Delta \mathrm{p}_{\mathrm{cond}}=\Delta \mathrm{p}_{\mathrm{cw}}
$$

The pressure drop through the piping is calculated in the same manner as that for the evaporator. The condenser water pipe length is estimated based on the height of the building, assuming that the cooling tower is on the roof.

$$
\mathrm{L}_{\text {pipe }}=2 \cdot \mathrm{H}_{\text {building }}
$$

The effective pipe length is longer than the actual length due to various flow obstacles.

$$
\mathrm{L}_{\text {pipe,eff }}=1.5 \cdot \mathrm{L}_{\text {pipe }}
$$

The head loss is assumed to be $4 \mathrm{ft}$ per $100 \mathrm{ft}$ of effective length.

$$
\text { head }_{\text {pipe }}=0.04 \cdot \mathrm{L}_{\text {pipe,eff }}
$$

The hydrostatic head $\left(\Delta \mathrm{p}_{\text {pipe,tower }}\right)$ is calculated based on the assumption that the cooling tower spray nozzles are $15 \mathrm{ft}$. higher than the tower basin. This corresponds to an added pressure supply requirement.

$$
\Delta \mathrm{p}_{\text {pipe,tower }}=\rho_{\mathrm{cw}} \cdot \mathrm{g} \cdot\left(\operatorname{head}_{\text {pipe }}+\text { head }_{\text {tower }}\right)
$$


where $\rho_{c w}$ is the density of the condenser water, and $g$ is the acceleration due to gravity.

The cooling tower nozzles are assumed to have a 20 psi pressure drop across them, a reasonable number for the type of nozzles that are used in a typical cooling tower of this size (Spraying Systems Co., Industrial Spray Products Catalog, 1993).

$$
\Delta \mathrm{p}_{\text {nozzle }}=20 \mathrm{psi}
$$

The overall pressure drop the pump is required to overcome on the condenser water-side is the sum of the individual pressure drops calculated for the condenser, condenser water piping, and the cooling tower spray nozzles.

$$
\Delta \mathrm{p}_{\text {pump }}=\Delta \mathrm{p}_{\text {cond }}+\Delta \mathrm{p}_{\text {pipe,tower }}+\Delta \mathrm{p}_{\text {nozzle }}
$$

The condenser water pump selected for this application provides a total pressure head of 41 psi.

\subsubsection{Performance}

The system model assumes that the chilled water pump has an isentropic efficiency of $80 \%$ ( $\left.\eta_{\text {pump }}\right)$, based on a Bell and Gossett Model $15106 G$ pump. Because the condenser water has a higher volumetric flow rate than the chilled water due to the higher load on the condenser, there is a larger pump used to handle the condenser water loop. The model assumes that the condenser water pump has an isentropic efficiency of 85\%, based on Bell and Gossett Model 151086 pump (Bell and Gossett Pump Selection (online), 2003). Figures 38 and 39 show where the cycle requirements fall on the pump curves for the selected pumps. 


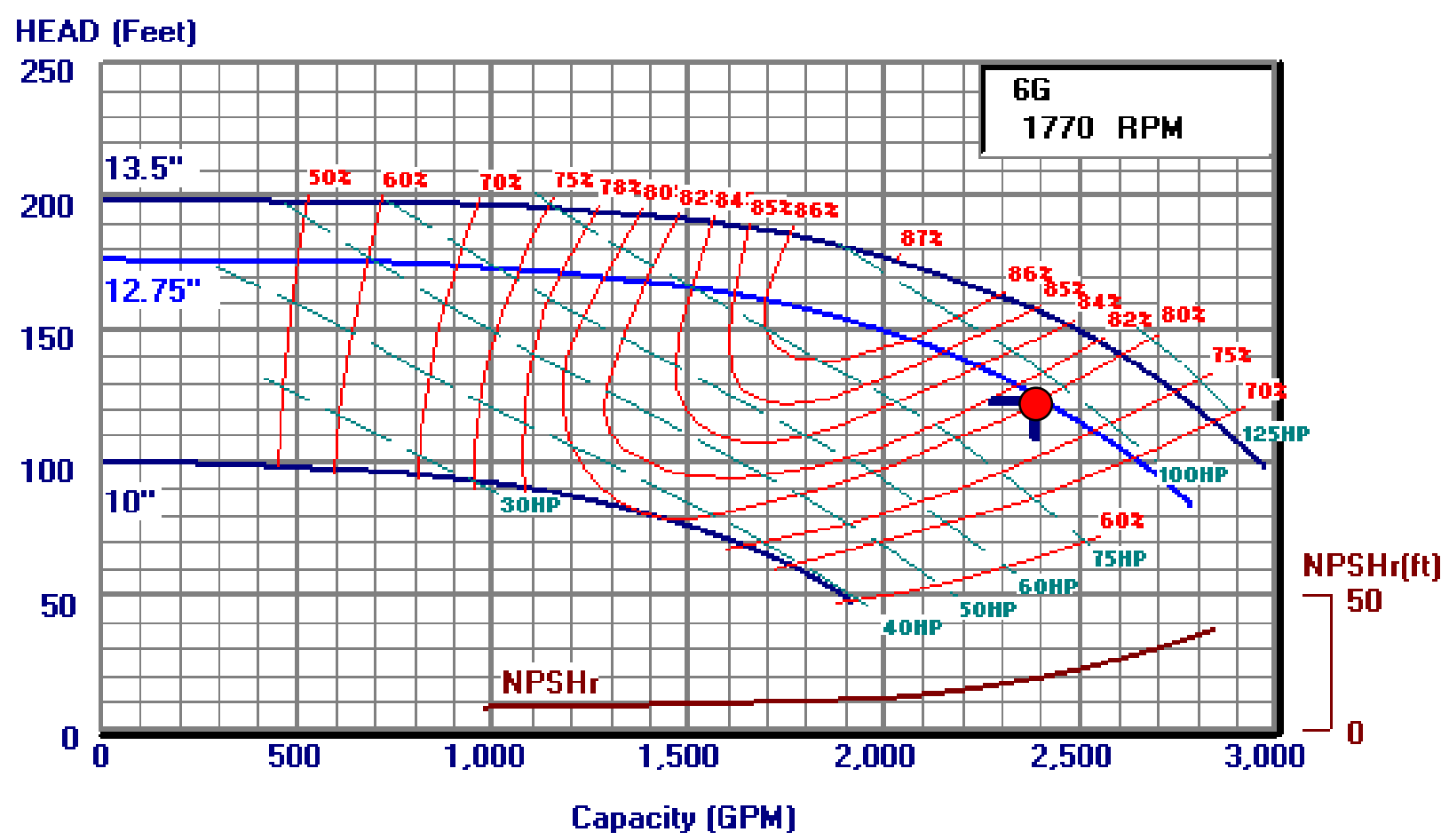

Figure 38 Pump curve illustrating the chilled water pump operating conditions for the Model $15106 G$ (courtesy of Bell and Gossett, 2003).

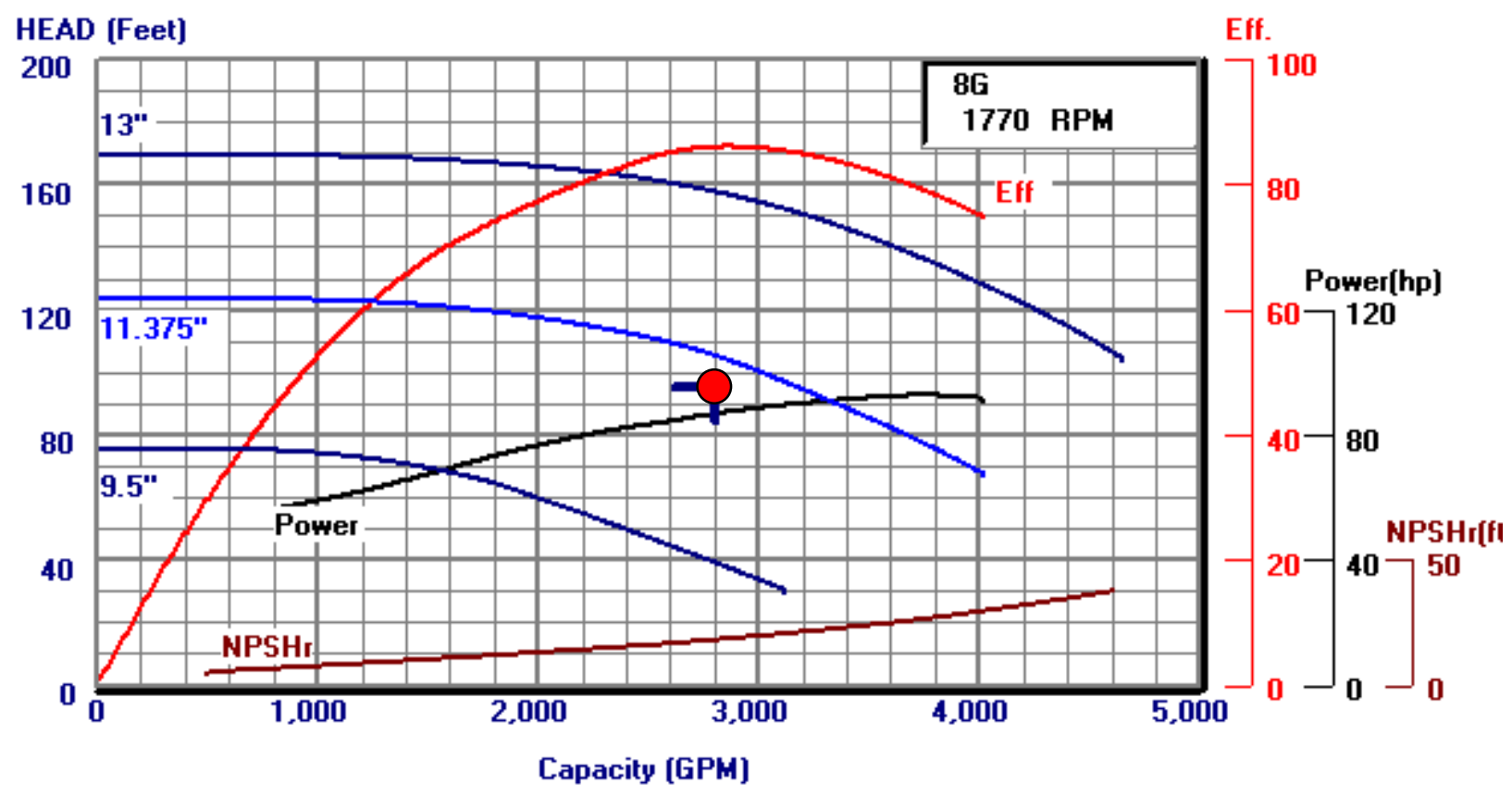

Figure 39 Pump curve illustrating the condenser water pump operating for the Model $15108 G$ (courtesy of Bell and Gossett, 2003). 
In order to determine the exact required pump work, the inlet and ideal exit conditions must be determined. The inlet enthalpy and entropy is defined by the chilled water return temperature $\left(54^{\circ} \mathrm{F}\right)$, and atmospheric pressure.

$$
\begin{aligned}
& \mathrm{h}_{\text {in }}=\operatorname{enthalpy}\left(\text { Water }, \mathrm{T}=54^{\circ} \mathrm{F}, \mathrm{p}=\mathrm{p}_{\text {atm }}\right) \\
& \mathrm{s}_{\text {in }}=\operatorname{entropy}\left(\text { Water }, \mathrm{T}=54^{\circ} \mathrm{F}, \mathrm{p}=\mathrm{p}_{\text {atm }}\right)
\end{aligned}
$$

The ideal (isentropic) exit condition is calculated using the required exit pressure and the inlet entropy.

$$
\mathrm{h}_{\mathrm{is}, \text { out }}=\text { enthalpy }\left(\text { Water, } \mathrm{p}=\mathrm{p}_{\mathrm{atm}}+\Delta \mathrm{p}_{\mathrm{pump}}, \mathrm{s}=\mathrm{s}_{\mathrm{in}}\right)
$$

The actual exit enthalpy is determined using the definition of isentropic efficiency.

$$
\eta_{\text {pump }}=\frac{\mathrm{h}_{\text {is,out }}-\mathrm{h}_{\text {in }}}{\mathrm{h}_{\text {out }}-\mathrm{h}_{\text {in }}}
$$

The mass flow rate of the chilled water $\left(\dot{\mathrm{m}}_{\text {chw }}\right)$ is calculated in the evaporator model using an energy balance. The required pump power is determined using the chilled water flow rate and the required enthalpy rise in the following equation.

$$
\dot{\mathrm{W}}_{\text {pump }}=\dot{\mathrm{m}}_{\text {chw }} \cdot\left(\mathrm{h}_{\text {out }}-\mathrm{h}_{\text {in }}\right)
$$

The same method is used to calculate the condenser water pump power requirement.

For an R-134a chiller, the pump power is a significant penalty on the system. The required power is approximately $92.2 \mathrm{hp}$ for the chilled water pump. Bell and Gossett matches a $100 \mathrm{hp}$ motor to this pump. The required for the condenser water pump is approximately $78.4 \mathrm{hp}$. Bell and Gossett matches this pump to a $100 \mathrm{hp}$ motor as well. The combination of chilled and condenser water pumps corresponds to approximately $18 \%$ of the total required power of the cycle. These calculated required pump powers match up well with the plots in Figures 38 and 39. 


\subsubsection{Cost}

There are two components to the cost for the water pumping systems: the cost of the pumps, and the cost of the energy required to run the pumps. There are two pumps required for the system, the chilled water pump and the condenser water pump. The chilled water pump, a Bell and Gossett model 1510 6G, costs $\$ 5950$. The condenser water pump, a Bell and Gossett model $15108 \mathrm{G}$, costs $\$ 6850$. The condenser water pump costs more than the chilled water pump due to its higher required flow rate, and head requirement. The energy cost of running the pumps is calculated for each cycle configuration in Chapter 5.

\subsection{Baseline, Water System Models}

Many of the baseline water system models are based on the same basic calculations developed for the single-stage R-134a cycle. The differences between the common component models for R-134a and water are described in Sections 4.2.1 through 4.2.3. There are also configuration possibilities that are not possible with the baseline R134a cycle. These include the use of direct-contact heat exchangers and intercooling. The models for these additional components are described in detail in Sections 4.2.4 through 4.2.6 and 4.2.8, where the last section pertains to purging, which is a penalty that is a consequence of using direct-contact heat exchangers.

\subsubsection{Compressor Model}

Water will not work in the same cycle configuration described for R-134a in Section 4.1, due to the higher required pressure ratio and volumetric flow rates at the compressor inlet. Current compressor technology does not permit a single stage 
centrifugal compressor with volumetric flow rates exceeding 150 times that for R-134a and a pressure ratio approaching 6. For this reason, staging the compression process is essential. Despite the inherent added expense of having more than one compressor, there are advantages to multistaging. Between compression stages, high temperature discharge vapor can be cooled from a superheated vapor back to near the vapor dome prior to entering the downstream compressor. This is known as intercooling. Figure 40 illustrates the T-s diagram of a flash intercooled and non-intercooled cycle. Notice the reduction in the area enclosed by the T-s diagram representing a reduction in the power consumption.

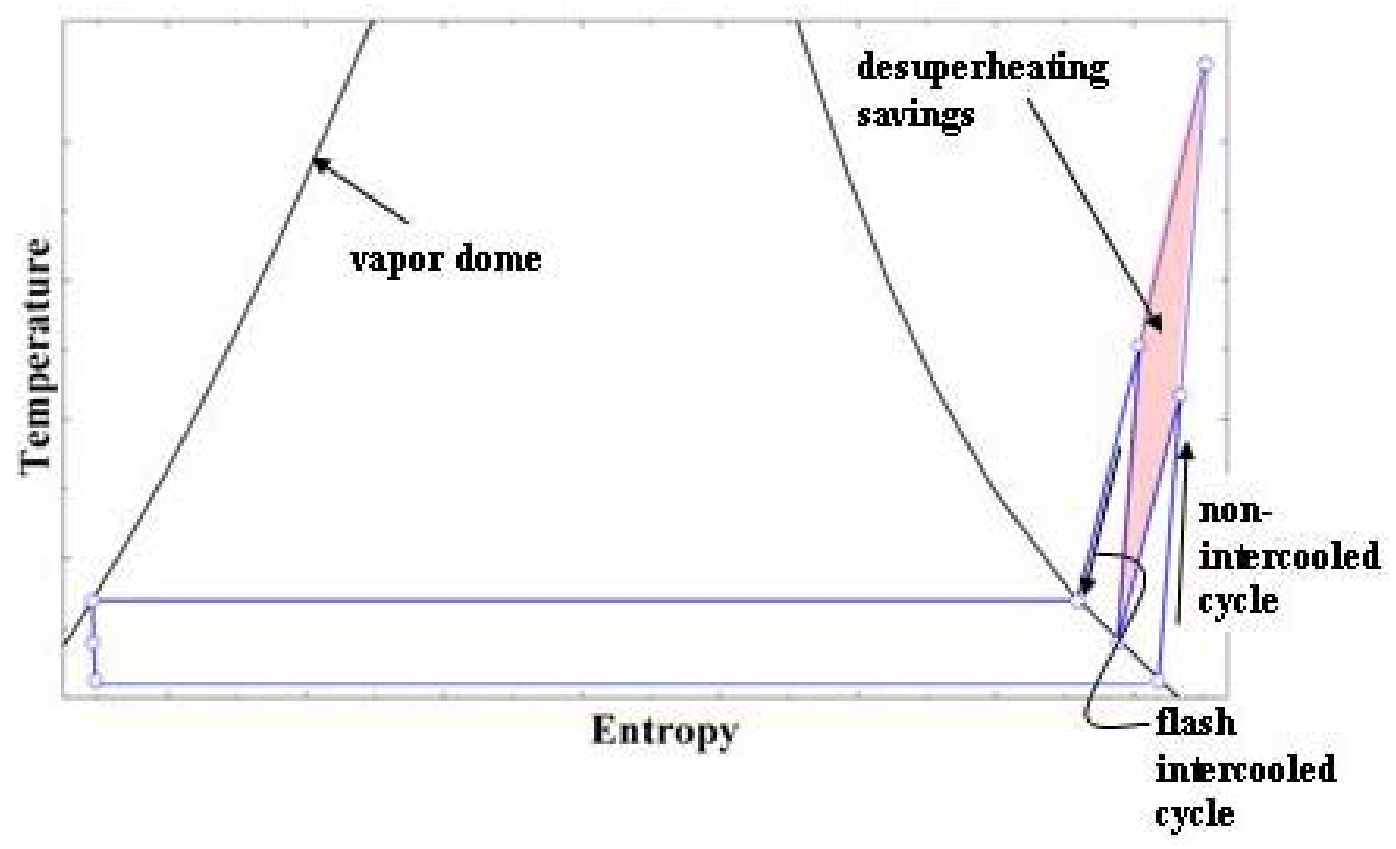

Figure 40 T-s diagram illustrating the savings of flash intercooling.

The various methods of intercooling were explored in detail in Chapter 3. The intercooler model is described in Section 4.2.6.

With water as a refrigerant, the two required compressors complicate the optimal cycle analysis. In particular, there is no clear answer to the question of how to divide the compression stages. An equal pressure ratio scheme was chosen in this report. Dividing 
the compression process into two processes of equal pressure ratio, however, does not ensure that the compressors will have the same size or performance. The high pressure compressor has a lower volumetric flow rate due to the greater gas density of the inlet vapor (see Table 12); however, this compressor requires more power because the absolute pressure rise that is must develop is larger than that of the low pressure compressor and therefore this compressor is penalized more significantly by the (assumed) fixed loss factors applied to the pressure recovery flow devices (e.g., the diffuser and vaneless space).

Table 12 Density and volumetric flow rates for the high and low pressure compressors using equal pressure ratio staging.

Low pressure compressor Inlet vapor density Inlet vapor volumetric flow rate High pressure compressor $\quad 1.09\left(10^{-3}\right) \mathrm{lbm} / \mathrm{ft}^{3} \quad 191,800 \mathrm{cfm}$

From a performance standpoint, it would be ideal to have different compressors that are perfectly matched to the operating conditions. To decrease manufacturing cost, practical two-stage compression system will likely use two identical compressors possibly running on the same shaft and therefore at identical speeds. In this case, both compressors will have slightly off-design performance characteristics. The compressor model developed for this project was intended to describe a centrifugal compressor operating at its design condition. It is therefore not possible to use this model as a direct simulation tool in order to determine the effect of operating two identical compressors slightly off-design. However, this is a conservative model in the sense that it overestimates the performance that can be achieved using a water cycle and therefore inclusion of this effect will only further degrade the economic viability of the water cycle. 
The following section is a description of the centrifugal compressor model used to determine the performance, size, and cost of a steam compressor in a water-based vapor compression refrigeration cycle.

\subsubsection{Performance}

The analysis of the effect of the cycle configurations described in Chapter 3 showed that 2-stages of compression is likely optimal if one considers the balance between capital cost against the reasonable expectations of compressor capacity. The marginal benefit in operating costs is not likely to justify the capital cost associated with additional compressor impellers. The operating conditions of the centrifugal compressors is similar in that the inlet conditions are both very close to a saturated vapor for a flash intercooled cycle; the only significant difference between the stages is that the highpressure compressor works with lower volumetric flows despite a higher mass flow due to intercooling. The centrifugal compressor model for water as a refrigerant is essentially identical to the R-134a compressor model. For a detailed description of the calculation process for the centrifugal compressor model, refer to Section 4.1.1.1.

For a pair of centrifugal compressors with water as the refrigerant, the performance parameters for the subcomponents that make up the compressor are assumed to be the same as to those used for the R-134a. The justification for maintaining the same performance metrics is related to examining the Reynolds number of each subcomponent for each of the refrigerants. Table 13 shows that the appropriate Reynolds numbers are within approximately a factor of 2 for the two refrigerants which suggests that the flow behavior within these devices and their fundamental performance will likely not change significantly. Therefore, there was no adjustment of the nozzle efficiency, the 
impeller diffusion coefficient, the impeller efficiency, the efficiency of the vaneless space, and the vaned diffuser coefficient of pressure recovery.

Table 13 Reynolds numbers for centrifugal compressor components with different

\begin{tabular}{|c|c|c|c|}
\hline refrigerants. & & & \\
\hline & $\mathbf{R e}_{\mathrm{R}-134 \mathrm{a}}$ & $\operatorname{Re}_{\mathrm{H} 2 \mathrm{O} \text { vap }}(1)$ & $\mathrm{Re}_{\mathrm{H} 2 \mathrm{O} \text { vap }}(2)$ \\
\hline Nozzle & 73359 & 102,000 & 161,600 \\
\hline Impeller & 52894 & 35,400 & 55,600 \\
\hline Diffuser & 108964 & 33,700 & 52,500 \\
\hline
\end{tabular}

Figure 41 provides a closer look at the T-s diagram corresponding to the states and processes associated with the steam moving through the low pressure centrifugal compressor.

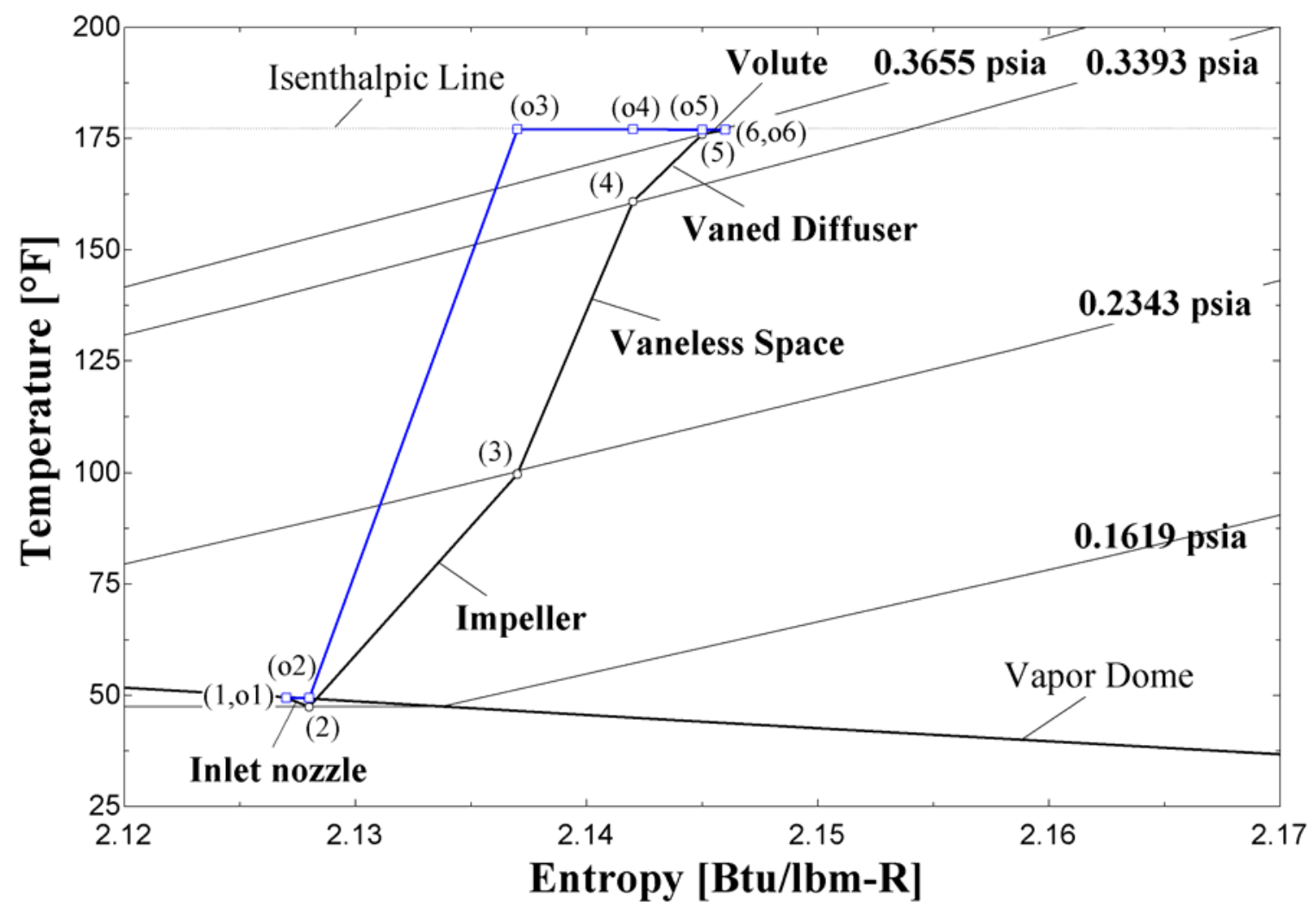

Figure 41 T-s diagram of water vapor centrifugal compressor.

\subsubsection{Size}

The centrifugal compressors required for a water vapor compression refrigeration cycle are significantly larger than a single compressor for an R-134a cycle operating 
under the same conditions. By dividing the compression process up into two-stages, the compression ratio for each water vapor compressor is slightly smaller than the R-134a compressor. Table 14 is a comparison of the size and performance of the two water vapor compressors to the single R-134a compressor.

Table 14 Size and performance of centrifugal compressors for water and R-134a cycles.

\begin{tabular}{|l|l|l|}
\hline & Water & R-134a \\
\hline $\mathbf{1}^{\text {st }}$ stage & & \\
- tip diameter & $110 \mathrm{in}$ & $12.23 \mathrm{in}$ \\
- vol. flow rate & $415,200 \mathrm{cfm}$ & $2,770 \mathrm{cfm}$ \\
- inlet pressure & $0.142 \mathrm{psia}$ & $52.51 \mathrm{psia}$ \\
- pressure ratio & 2.40 & 2.58 \\
- power & $338 \mathrm{hp}$ & $739 \mathrm{hp}$ \\
- rotational speed & $2,960 \mathrm{rpm}$ & $10,000 \mathrm{rpm}$ \\
- tip Mach number & 1.01 & 1.17 \\
\hline $\mathbf{2}^{\mathbf{2} \text { s }}$ stage & & \\
- tip diameter & $73.7 \mathrm{in}$ & \\
- vol. flow rate & $191,800 \mathrm{cfm}$ & \\
- inlet pressure & $0.341 \mathrm{psia}$ & \\
- pressure ratio & 2.40 & \\
- power & $375 \mathrm{hp}$ & \\
- rotational speed & $4,535 \mathrm{rpm}$ & \\
- tip Mach number & 1.01 & \\
\hline - total power & $714 \mathrm{hp}$ & $739 \mathrm{hp}$ \\
\hline
\end{tabular}

Although the pressure ratios of the water vapor compressors and the R-134a compressor are nearly identical, it can be seen that the tip diameters are not similar for any of the three compressors. The volumetric flow rate is a better scaling factor for the tip diameter. As a first approximation, the square of the tip diameter is approximately proportional to the volumetric flow rate.

\subsubsection{Investigation of Wet Compression}

One potential problem with a water-based refrigeration cycle arises when saturated vapor is introduced to the compressor, there is a possibility of two-phase flow at the exit of the compressor nozzle. This phenomenon may cause droplets of water to form 
and impact compressor impeller blades resulting in erosion and reducing reliability. The thermodynamics that lead to this situation are discussed more in Section 4.1.1.1 where the compressor model is developed. This is a concern that is also found in the power generation industry where the exit of a steam power turbine may be two-phase. Data has shown that (in a steam power plant) the steam will remain vapor in a non-equilibrium, supersaturated state provided that the nozzle causes the static enthalpy to drop less than $\sim 60 \mathrm{Btu} / \mathrm{lbm}(140 \mathrm{~kJ} / \mathrm{kg})$ below the saturation enthalpy at the corresponding pressure. Any additional decrease in the static enthalpy causes the flow to undergo a condensation shock and results in increased levels of blade erosion. Figure 42 illustrates the imaginary line that delineates the supersaturated and condensed states: the Wilson line (El Wakil, 1984). The situation in a water refrigeration cycle is similar and a similar behavior is expected in the compressor nozzle when a water compressor operates on saturated or near-saturated water vapor. Provided that the exit to the compressor inlet nozzle is above the Wilson line, no liquid water is expected on the blades and the associated potential erosion problems should be avoided. 


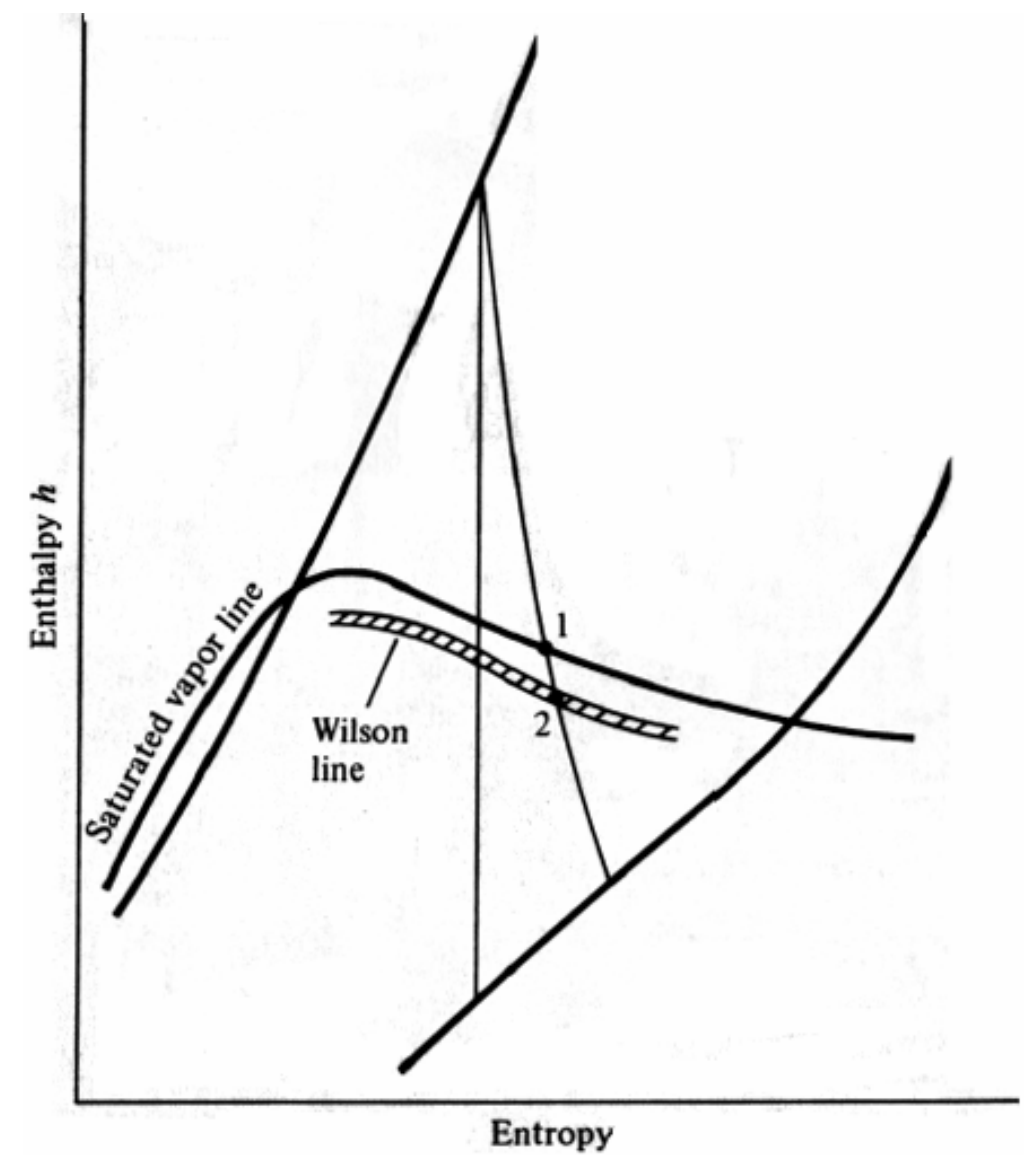

Figure 42 Mollier diagram illustrating the Wilson line (from El Wakil, 1984).

The amount of superheat necessary to keep the compressor nozzle exit outside the vapor dome is illustrated in Figure 43. For a 2-stage flash intercooled cycle with water as a refrigerant, the compressor requires $\sim 4.7^{\circ} \mathrm{F}\left(2.6^{\circ} \mathrm{C}\right)$ of superheat in order to stay out of the vapor dome and therefore ensure that condensation will not occur. Figure 43 also shows how the COP varies with superheat. As expected the performance drops with added superheat. 


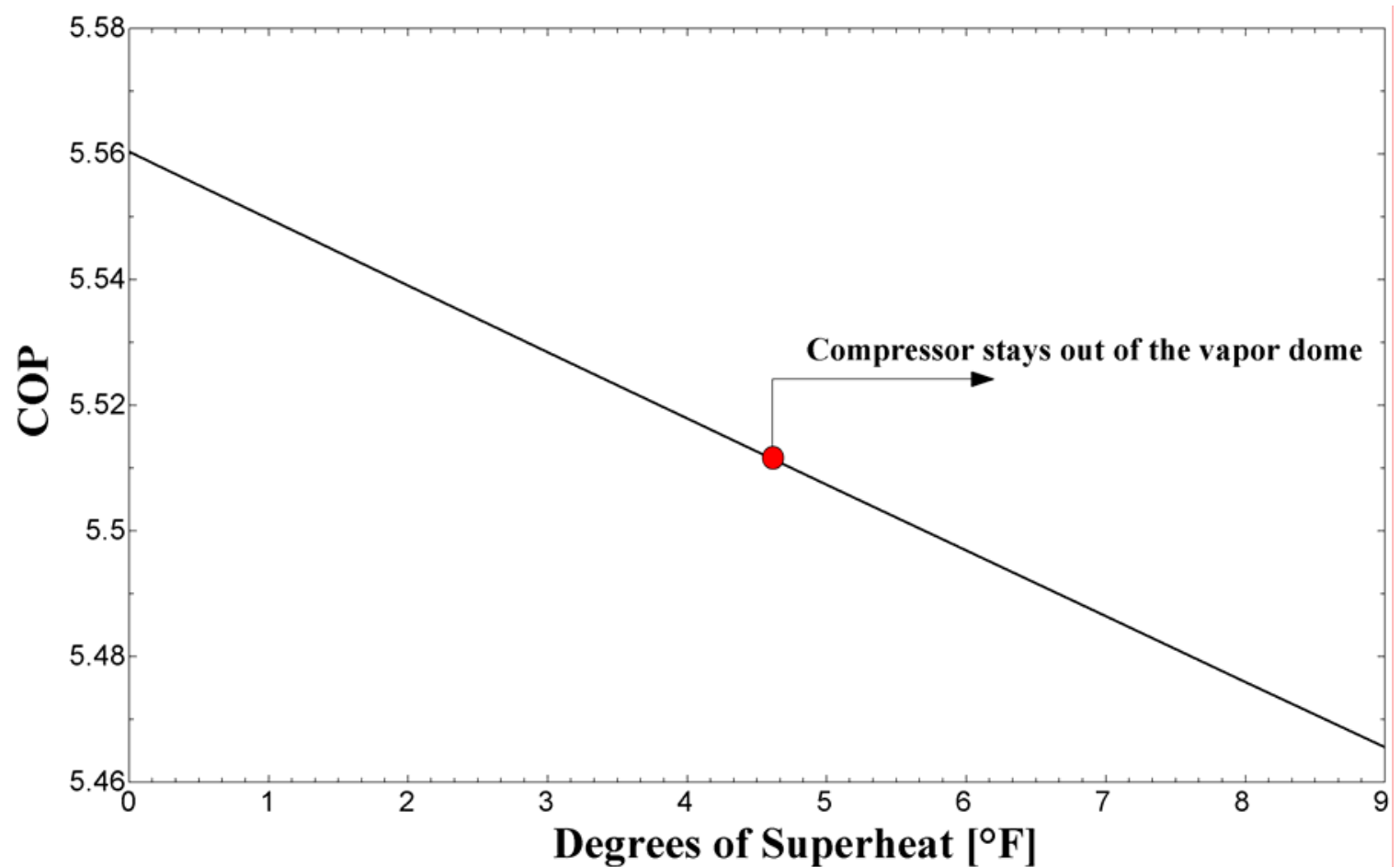

Figure $43 \mathrm{COP}$ vs. compressor suction superheat for a 2-stage flash intercooled cycle.

\subsubsection{Cost}

The cost of a centrifugal compressor is estimated based on the fixed costs associated with mass production ( $\mathrm{R} \& \mathrm{D}$, labor, overhead, etc.) and the material costs based on the square of the impeller tip diameter. The formula developed for the R-134a centrifugal compressor is used without modification for the water compressors. The centrifugal cost data is based on information from U.S. chiller manufacturers and is therefore associated with R134a compression technology. Applying these cost curves to the water compressors is a significant extrapolation of this data.

$$
\text { Material Cost }=\left(\frac{\$ 25.75}{\mathrm{in}^{2}}\right) \cdot \mathrm{d}_{\text {tip }}^{2}+\left(\frac{\$ 765}{\mathrm{in}}\right) \cdot \mathrm{d}_{\text {tip }}
$$

Table 15 summarizes the cost of the two water vapor compressors and compares them to the cost of the single R-134a compressor. 
Table 15 Cost of centrifugal compressors using water as a refrigerant.

\begin{tabular}{l|c|r} 
& Tip diameter [in] & Cost [\$] \\
\hline L.P. Compressor & 110 & 662,700 \\
H.P. Compressor & 74 & 326,800 \\
\hline & Total & 989,500 \\
\hline R-134a Compressor & 12.23 & 22,000
\end{tabular}

\subsubsection{Indirect-Contact Condenser Model}

The indirect condenser model for water as a refrigerant is similar to the indirect heat exchanger model developed for the R-134a cycle. The indirect condenser model uses the same ARTI specified conditions as a basis to determine all of the thermodynamic end-states. The model also has some flexibility with regard to geometric and hydrodynamic limits as it solves the appropriate heat transfer equations in order to establish the required heat exchanger thermal performance. Table 16 notes the significant difference between compressor discharge temperatures for the R-134a and water refrigeration cycles.

Table 16 Comparison between discharge temperatures for water and R-134a as refrigerants.

\begin{tabular}{c|c|c} 
& Two-stage water cycle & Single-stage R-134a cycle \\
\hline L.P Compressor & $207^{\circ} \mathrm{F}$ & $110^{\circ} \mathrm{F}$ \\
H.P. Compressor & $238^{\circ} \mathrm{F}$ &
\end{tabular}

\subsubsection{Performance}

The size of the heat exchanger is principally determined by the heat transfer coefficients on the tube- and the shell-side. When considering the use of water in a vapor compression refrigeration cycle operating at the ARTI specified conditions, it becomes important to consider refrigerant side pressure drop. If the required thermal performance leads to a heat exchanger size that results in large refrigerant-side pressure drop, the overall performance of the refrigeration system will be negatively impacted. Water- 
based refrigeration cycles are particularly sensitive to refrigerant-side pressure drop and consequently, particular attention needs to be paid to minimize this penalty on the system. There are geometric levers that can be exercised in an attempt to minimize the pressure drop such as the tube pitch. In contrast, refrigerant-side pressure drop is less of an issue in an R-134a condenser where the absolute pressure is upwards of 150 psia (10 atm) and the specific volume of the condensing vapor is much smaller. With water, the condenser operating pressure will be lower than 1 psia and the specific volume much higher, a combination that results in large pressure losses for a given flow rate and also makes the cycle more sensitive to pressure loss in general.

Figure 44 shows the variation of the refrigerant pressure drop with tube pitch. A pressure drop much greater than 0.01 psia is a maximum practical limit; above this value the COP begins to drop off dramatically. This small absolute pressure drop is important because the condenser pressure is only around 1 psia. To mitigate the effects of refrigerant-side pressure drop, the tube pitch is assumed to be greater than $\sim 3$ times the tube outer diameter. Pressure drop is an even more substantial problem in the evaporator, which will be discussed in Section 4.2.3. 


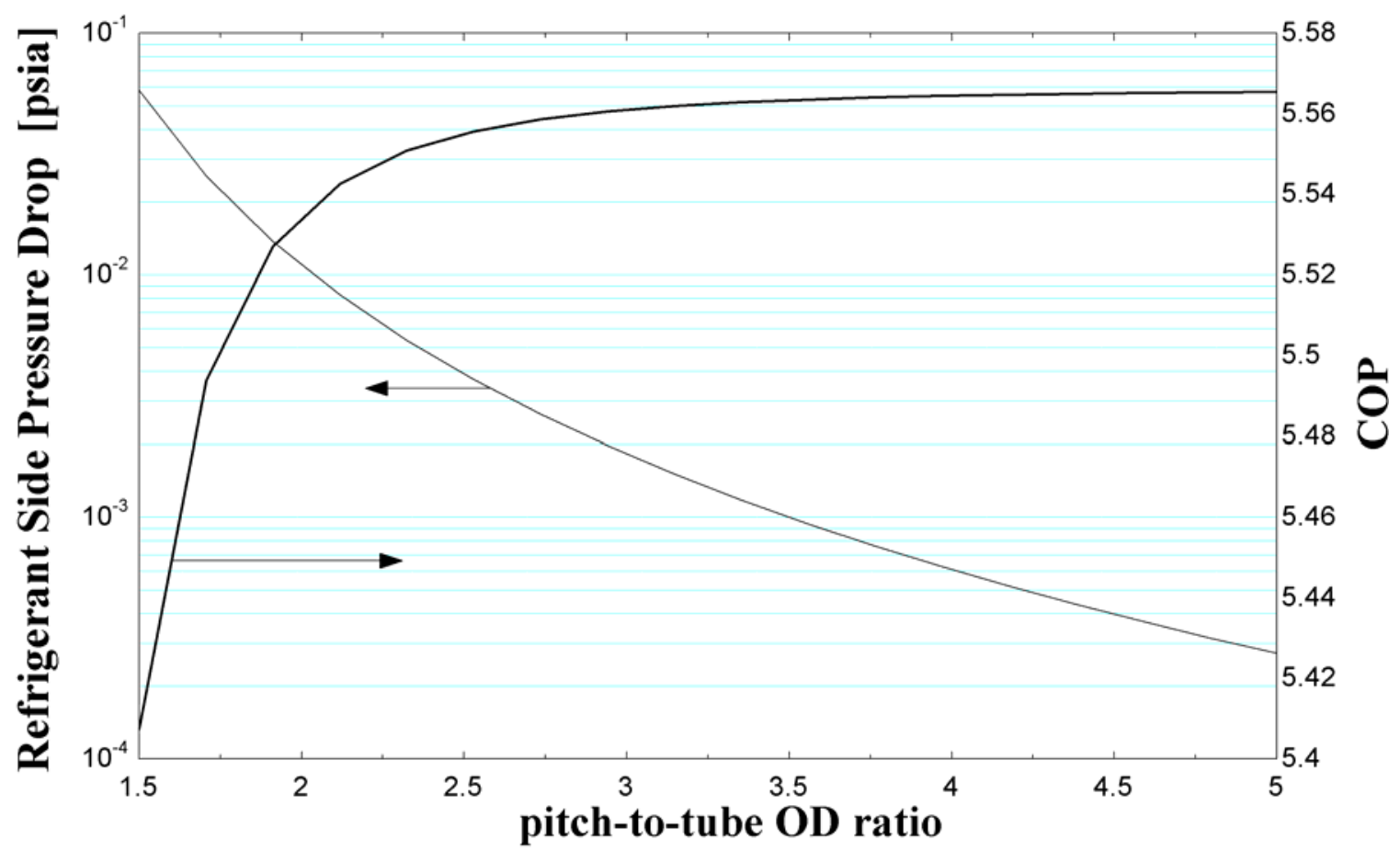

Figure 44 Plot of refrigerant pressure drop and COP variation with tube pitch.

Allowing the tube pitch to be large results in a low refrigerant-side pressure drop because it leads to a low refrigerant velocity. Low pressure drop also leads to a large heat exchanger with much of its internal volume not contributing to heat transfer. To illustrate this effect, Figure 45 shows the condenser shell diameter (a measure of the overall condenser size) over a range of varying pitch-to-tube OD ratios. The size of the condenser is almost a linear function of the pitch-tube OD ratio while the refrigerant-side pressure drop is a much stronger function of the pitch-tube OD ratio. The selection of an appropriate geometry for the condenser in a water-based refrigeration cycle must balance these competing effects. 


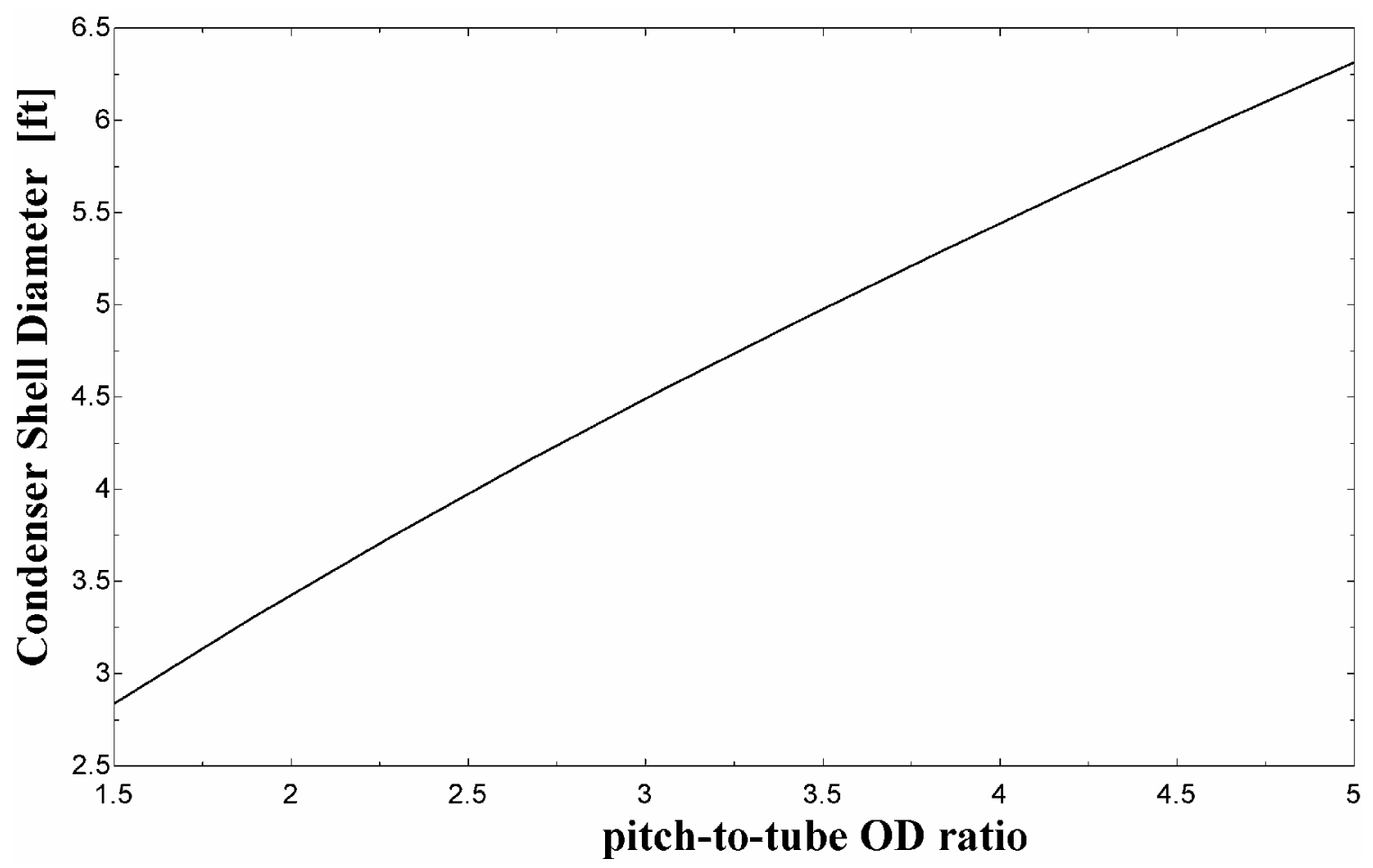

Figure 45 Plot of condenser shell diameter as a function of tube pitch.

The heat transfer coefficients for the tube-side (i.e., the cooling water side) are identical to those calculated for the R-134a condenser because the water velocity is specified. The heat transfer coefficients on the outside of the tubes differ based on the different thermophysical properties of the refrigerants but are calculated using the same correlations and applying the same correction factor to account for enhanced surfaces. These values are compared in Table 17, assuming the same tube pitch for both the R134a and steam condensers.

Table 17 Tube heat transfer coefficient comparison between water and R-134a.

\begin{tabular}{r|c|c} 
& Water $\left[\mathbf{B t u} / \mathbf{h}-\mathbf{f t}^{2}-\mathbf{R}\right]$ & $\mathbf{R}-\mathbf{1 3 4 a}\left[\mathbf{B t u} / \mathbf{h}-\mathbf{f t}^{2}-\mathbf{R}\right]$ \\
\hline Outside & 10,100 & 2,754 \\
Inside & 1,500 & 1,500
\end{tabular}

Table 17 indicates that if pressure drop were not considered, the condenser in a water-based refrigeration cycle would actually be slightly smaller than a comparable R134a condenser due to the higher refrigerant-side heat transfer coefficient. However, as 
previously discussed, the water-based refrigerant condenser has a much greater sensitivity to pressure drop and so tube pitch takes on a key role and increases the size of the heat exchanger.

\subsubsection{Size}

In order to minimize the refrigerant-side pressure drop in the water-based refrigeration cycle condenser, the tube pitch specification is increased from 1.5 (as with $\mathrm{R}-134 \mathrm{a})$ to 3 times the tube outer diameter. This larger tube pitch corresponds to a refrigerant-side pressure drop of just 0.001 psi or $\sim 2.6 \%$ of the total inlet refrigerant pressure. Table 18 shows the resulting dimensions and tube specifications of the shelland-tube condenser in the water-based refrigeration cycle.

Table 18 Indirect-contact condenser dimensions based on ARTI standard conditions for

\begin{tabular}{l|l|l|l} 
Dimension & Symbol & Water & R-134a \\
\hline Heat exchanger diameter & $\mathrm{D}_{\mathrm{HX}}$ & $66 \mathrm{in}$ & $36 \mathrm{in}$ \\
Heat exchanger length & $\mathrm{L}_{\mathrm{HX}}$ & $24 \mathrm{ft}$ & $13 \mathrm{ft}$ \\
Number of tubes & $\mathrm{N}_{\text {tubes }}$ & 908 & 910 \\
Tube pitch & Pitch & $2.25 \mathrm{in}$ & $1.125 \mathrm{in}$ \\
Approach temperature difference & $\Delta \mathrm{T}_{\text {approach }}$ & $0.4^{\circ} \mathrm{F}$ & $1.41^{\circ} \mathrm{F}$
\end{tabular}

The condenser for the water refrigeration cycle has a lower approach temperature and therefore performs better. This approach temperature was found to be nominally the economic optimal one in Chapter 5.

\subsubsection{Cost}

Like the R-134a condenser, the cost of an indirect-contact condenser is assumed to scale with size and the material cost is divided into two main parts, the shell cost and the tube cost. The shell cost is assumed to be a function of the volume enclosed by the shell $\left(\mathrm{V}_{\text {shell }}\right)$ in cubic feet. The total tube cost is assumed to be a function of the total tube 
length. The formula used to determine the cost of the shell and tubes for the R-134a heat exchangers is used without modification for the water cycle condenser. The following equations determine the shell cost in terms of $\$ / \mathrm{ft}^{3}$ enclosed and the tube cost in terms of $\$ / \mathrm{ft}$. The fixed costs associated with fabricating shell-and-tube condensers on a mass scale are approximately $60 \%$ of the sum of the shell and tube costs.

$$
\begin{gathered}
\text { Shell Cost }=\left(\frac{\$ 25.50}{\mathrm{ft}^{3}}\right) \cdot \frac{\pi}{4} \cdot \mathrm{D}_{\mathrm{HX}, \mathrm{ft}}^{2} \cdot \mathrm{L}_{\mathrm{HX}, \mathrm{ft}} \\
\text { Tube Cost }=\left(\frac{\$ 0.76}{\mathrm{ft}}\right) \cdot \mathrm{N}_{\text {tubes }} \cdot \mathrm{L}_{\mathrm{HX}, \mathrm{ft}}
\end{gathered}
$$

Table 19 shows how the shell cost and the tube costs compare for the water and the R134a cycle.

Table 19 Water and R-134a condenser cost comparison.

\begin{tabular}{l|c|c} 
& Two-stage water cycle & Single-stage R-134a cycle \\
\hline Shell Cost & $\$ 14,600$ & $\$ 2,330$ \\
Tube Cost & $\$ 16,500$ & $\$ 9,000$ \\
\hline Total Cost* & $\mathbf{\$ 4 9 , 5 0 0}$ & $\mathbf{\$ 1 8 , 3 0 0}$
\end{tabular}

\subsubsection{Indirect-Contact Evaporator Model}

The indirect evaporator model developed for the R-134a cycle forms the basis for the evaporator model used with the water cycle. In the same way that the condenser is very sensitive to pressure drop, the evaporator is approximately an order of magnitude more sensitive to the same pressure drop because the operating pressure in the waterbased cycle evaporator is much less, approximately 0.13 psia.

The evaporator for the water refrigeration cycle cannot operate in a flooded mode due to the cycle's sensitivity to hydrostatic head. Therefore, the heat transfer coefficient is calculated assuming falling film boiling over a horizontal tube bundle as opposed to 
saturated pool boiling. The following section is a description of the differences of the water cycle pressure drop and heat transfer coefficient calculations, relative to the R-134a cycle.

\subsubsection{Performance}

With the modifications to the evaporator model corresponding to the water vapor/liquid conditions on the shell side, the functionality of the evaporator model is largely the same as that for R-134a. Using the assumption that the bulk of the volume of fluid moving through the evaporator is in the vapor state implies that the pressure drop equations must be based on the vapor density and viscosity rather than liquid density and viscosity as in the flooded configuration. Thus, the refrigerant-side pressure drop is characterized by the vapor velocity as shown in the following equation for pressure drop through a tube bank (Incropera and DeWitt, 2002).

$$
\Delta \mathrm{p}_{\text {ref }}=\mathrm{N}_{\text {tube,vert }} \cdot \mathrm{N}_{\text {baffle }} \cdot \chi_{\text {tube, bank }} \cdot \mathrm{f}_{\text {tube,bank }} \cdot \rho_{\mathrm{v}, \text { ref }} \cdot \frac{\mathrm{v}_{\text {max,ref }}^{2}}{2}
$$

The maximum refrigerant velocity is based on the vapor density. Using mass conservation at the midpoint of the tube bundle, the maximum refrigerant velocity is determined.

$$
\mathrm{v}_{\text {max }, \text { ref }}=\frac{\dot{\mathrm{m}}_{\text {ref }}}{\rho_{\mathrm{v}, \text { ref }} \cdot \mathrm{L}_{\mathrm{HX}} \cdot \frac{\left(\mathrm{D}_{\mathrm{HX}}-\mathrm{N}_{\text {tube, vert }} \cdot \mathrm{d}_{\text {tube, } \mathrm{o}}\right)}{\mathrm{N}_{\text {baffle }}}}
$$

The heat transfer coefficient for the water cycle evaporator is calculated for laminar film boiling over horizontal tubes. This heat transfer coefficient correlation is related to the film Reynolds number $\left(\mathrm{Re}_{\mathrm{f}}\right)$. 


$$
\operatorname{Re}_{\mathrm{f}}=\frac{4 \cdot \Gamma}{\mu_{1, \mathrm{ref}}}
$$

where $\Gamma$ is defined as the mass flow rate divided by the perimeter of the tubes. Using the film Reynolds number, the heat transfer coefficient is calculated (Rohsenow, et al., 1998).

$$
\mathrm{htc}_{\mathrm{ref}}=\operatorname{Re}_{\mathrm{f}}^{-1 / 3} \cdot\left(\frac{4 \cdot \rho_{1, \mathrm{ref}}^{2} \cdot \mathrm{g} \cdot \mathrm{k}_{1, \mathrm{ref}}^{3}}{3 \cdot \mu_{1, \mathrm{ref}}^{2}}\right)^{1 / 3}
$$

The same correction factor used for the R134a evaporator to account for enhanced surfaces is applied here to the water evaporator. The evaporator heat transfer coefficients are compared for the water and R-134a cycles in Table 20.

\begin{tabular}{|c|c|c|}
\hline & Water & R-134a \\
\hline Outside, Btu/h-ft ${ }^{2}-\mathrm{R}$ & 1,670 & 1,540 \\
\hline Inside, Btu/h-ft' ${ }^{2}-R$ & 1,270 & 1,270 \\
\hline
\end{tabular}

Table 20 Evaporator tube heat transfer coefficient comparison.

The heat transfer coefficients for the tube-side (chilled water-side) are identical because the chilled water velocity is specified. The controlling heat transfer coefficient is on the shell-side (refrigerant-side).

\subsubsection{Size}

The indirect-contact evaporator for water is larger than the same component for the R-134a system. Table 21 is a comparison between the condenser sizes for both water and R-134a as refrigerants.

Table 21 Comparison of water and R-134a evaporators.

\begin{tabular}{l|l|l|l} 
Dimension & Symbol & Water & R-134a \\
\hline Heat exchanger diameter & $\mathrm{D}_{\mathrm{HX}}$ & $62 \mathrm{in}$ & $42 \mathrm{in}$ \\
Heat exchanger length & $\mathrm{L}_{\mathrm{HX}}$ & $20.5 \mathrm{ft}$ & $14 \mathrm{ft}$ \\
Number of tubes & $\mathrm{N}_{\text {tubes }}$ & 688 & 690 \\
Tube pitch & Pitch & $1.5 \mathrm{in}$ & $1.05 \mathrm{in}$ \\
Approach temperature difference & $\Delta \mathrm{T}_{\text {evap }}$ & $0.3^{\circ} \mathrm{F}$ & $1.23^{\circ} \mathrm{F}$
\end{tabular}


The evaporator for water as a refrigerant is approximately three times larger by volume than that for the R-134a evaporator. Again, notice that the performance of the water evaporator is higher as this approach temperature difference is found to be the most economically attractive in Chapter 5.

\subsubsection{Cost}

Like the R-134a evaporator, the cost of an indirect-contact evaporator is assumed to scale with its size and the material cost is divided into two main parts, the shell cost and the tube cost. The shell cost is assumed to be a function of the volume enclosed by the shell $\left(\mathrm{V}_{\text {shell }}\right)$ in cubic feet. The total tube cost is assumed to be a function of the total tube length. The formula used to determine the cost of the shell and tubes for the R-134a heat exchangers is used without modification for the water cycle evaporator. The following equations determine the shell cost in terms of $\$ / \mathrm{ft}^{3}$ enclosed and the tube cost in terms of $\$ / \mathrm{ft}$. The fixed costs associated with fabricating shell-and-tube evaporators on a mass scale are approximately $60 \%$ of the sum of the shell and tube costs.

$$
\begin{gathered}
\text { ShellCost }=\left(\frac{\$ 22.20}{\mathrm{ft}^{3}}\right) \cdot \frac{\pi}{4} \cdot \mathrm{D}_{\mathrm{HX}, \mathrm{ft}}^{2} \cdot \mathrm{L}_{\mathrm{HX}, \mathrm{ft}} \\
\text { Tube Cost }=\left(\frac{\$ 0.91}{\mathrm{ft}}\right) \cdot \mathrm{N}_{\text {tubes }} \cdot \mathrm{L}_{\mathrm{HX}, \mathrm{ft}}
\end{gathered}
$$

Table 22 shows that economically, the indirect water cycle evaporator is a net penalty when compared with an R-134a evaporator. Thus, a direct-contact water cycle evaporator (not a possibility for an R-134a cycle) may have a significant economic benefit. 
Table 22 Water and R-134a evaporator cost comparison.

\begin{tabular}{l|c|c} 
& Two-stage water cycle & Single-stage R-134a cycle \\
\hline Shell Cost & $\$ 9,400$ & $\$ 2,990$ \\
Tube Cost & $\$ 12,800$ & $\$ 8,790$ \\
\hline Total Cost* & $\$ 37,600$ & $\$ 19,900$ \\
$*$ Total Cost includes fixed cost &
\end{tabular}

\subsubsection{Direct-Contact Condenser Model}

Water is widely used as a medium for transporting energy in refrigeration applications (e.g., it represents the cooling load or heat rejection medium). Typically, heat is absorbed or rejected from a refrigeration system indirectly using a heat exchanger. Because heat exchangers rely on a wall to physically separate the water from the refrigerant, the overall efficiency of the refrigeration system is diminished and capital cost increased. The extent to which the refrigeration system will experience an operating penalty will depend on the heat exchanger approach ${ }^{3}$.

An alternative to an indirect-contact heat exchanger is a direct-contact heat exchanger. In a direct-contact heat exchanger, the water is brought into intimate contact with the refrigerant; therefore, direct-contact heat exchangers are only applicable to a very few refrigeration cycles where the heat transfer fluid is compatible with or identical to the refrigerant. Synthetic refrigerants and alternative natural refrigerants are not likely candidates for direct-contact heat exchange due to possible environmental or toxicity concerns. The potential to use a direct-contact condenser provides a unique means for cycles using water as a refrigerant to recoup both capital and operating costs as directcontact heat exchangers are likely to be both less expensive and have higher performance than indirect-contact heat exchangers.

\footnotetext{
${ }^{3}$ The "approach" is defined as the minimum difference in temperature between the refrigerant and water in the heat exchanger.
} 
Direct-contact condensers are typically found in power generation facilities as direct feedwater heaters. The major difference between a direct feedwater heater and the direct-contact condenser in a cycle using water as a refrigerant is the operating pressure. Whereas the direct feedwater heater typically operates at pressures in excess of 10 atmospheres, the direct-contact condenser in a refrigeration cycle must operate pressures less than $1 / 20^{\text {th }}$ of an atmosphere (approximately 200x lower). This is a major issue relative to the pressure loss in the direct-contact heat exchanger which affects both the size and performance of these heat exchangers. Note that in power generation systems, the direct feedwater heater is typically the most convenient location to purge the noncondensables from the system, for reasons that are described in Section 4.2.8.

The following sections are a description of the direct-contact condenser model and a prediction of its performance, size and cost.

\subsubsection{Performance}

In a direct-contact heat exchanger, the refrigerant is brought into intimate contact with the secondary working fluid. For heat exchanger calculations, the water acting as a primary refrigerant will be referred to as the "refrigerant" and the water acting as a secondary refrigerant will be referred to as the "condenser water".

Figure 46 shows a direct-contact condenser as a cylindrical shell with a fill media (packing) as well as the locations of the various flows and the corresponding state numbers. Table 23 describes each of the states. 
Table 23 States in a direct-contact condenser.

\begin{tabular}{c|l} 
State & Location \\
\hline 1 & Refrigerant inlet \\
2 & Refrigerant exit \\
3 & Condenser water supply \\
4 & Condenser water (after throttle) \\
5 & Condenser water (before pump) \\
6 & Condenser water return
\end{tabular}

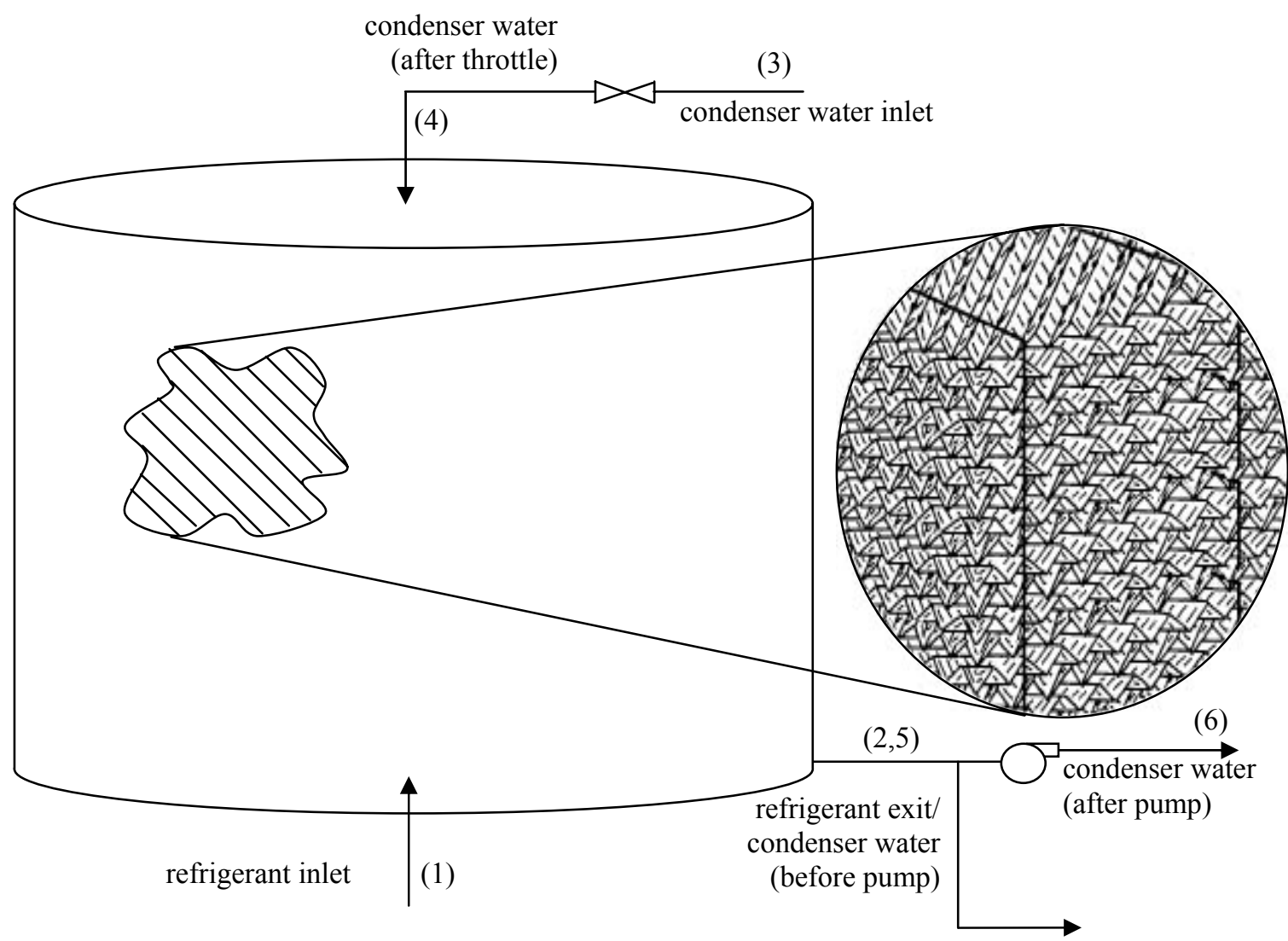

Figure 46 Schematic of direct-contact condenser with fill media.

The direct-contact condenser using fill media is modeled in this section. Initial modeling efforts focused on a direct-contact condenser in which no fill media was used; rather, heat and mass transfer occur by virtue of superheated discharge vapor being brought into direct-contact with very fine spray droplets of the cooler condenser water. The trajectory of a droplet was calculated based on the drag force exerted on the droplet by the vapor flow. The heat transfer characteristics of the droplets were determined based 
on the relative velocity between the droplets and the vapor. The droplet-to-vapor temperature difference requirement determined the time that the droplet had to fall in order to obtain a suitable residence time. This residence time requirement, coupled with the trajectory of the droplet based on the forces exerted on it, determined the length of the heat exchanger. Pressure drop considerations together with the spray characteristics of a nozzle (cone angle, droplet size, flow rate, etc.) determined the diameter of the heat exchanger.

The details of the "unfilled" direct-contact heat exchanger model are not reported here; the results of the model indicated that such a heat exchanger would be much too large to be practical. This conclusion was based, primarily, on the size of the droplets that could be achieved given the nozzle pressure available assuming practical pumping requirements. This conclusion is consistent with the descriptions of those cycles that use water as a refrigerant, included in the literature survey found in Chapter 2. Instead, a direct-contact heat exchanger using a porous, wetted packing material is considered here.

The refrigerant inlet state is calculated using the exit temperature and exit pressure predicted by the centrifugal compressor model. Note that these values are related to the cycle requirements and the characteristics of the heat exchanger; therefore, some iteration is required between the compressor and heat exchanger subprogram as well as the main cycle program.

$$
\begin{gathered}
\mathrm{T}_{1}=\mathrm{T}_{\text {condenser,in }} \\
\mathrm{p}_{1}=\mathrm{p}_{2}+\Delta \mathrm{p}_{\text {cond }}
\end{gathered}
$$

where $\Delta \mathrm{p}_{\text {cond }}$ is the direct-contact condenser pressure drop, which is based on the directcontact condenser geometry. 
The refrigerant inlet temperature and pressure are used to determine the remainder of the properties.

$$
\begin{aligned}
& \mathrm{h}_{1}=\operatorname{enthalpy}\left(\text { Steam, } \mathrm{T}=\mathrm{T}_{1}, \mathrm{p}=\mathrm{p}_{1}\right) \\
& \mathrm{s}_{1}=\operatorname{entropy}\left(\text { Steam, } \mathrm{T}=\mathrm{T}_{1}, \mathrm{p}=\mathrm{p}_{1}\right) \\
& \mathrm{v}_{1}=\operatorname{volume}\left(\text { Steam, } \mathrm{T}=\mathrm{T}_{1}, \mathrm{p}=\mathrm{p}_{1}\right)
\end{aligned}
$$

The refrigerant exit properties are found based on the exit pressure and quality. The exit pressure is a function of the inlet pressure and the pressure drop through the condenser, which is influenced by heat exchanger geometry. The condenser outlet state is assumed to be completely condensed with no subcooling and therefore the exit quality is zero.

$$
\begin{gathered}
\mathrm{T}_{2}=\mathrm{T}_{\text {condenser water, out }}+\Delta \mathrm{T}_{\text {cond }} \\
\mathrm{x}_{2}=0
\end{gathered}
$$

where $\Delta \mathrm{T}_{\text {cond }}$ is the direct-contact condenser approach temperature difference.

The pressure and quality are used to calculate the remainder of the state properties for the refrigerant exiting the direct-contact condenser.

$$
\begin{aligned}
& \mathrm{p}_{2}=\operatorname{pressure}\left(\text { Steam }, \mathrm{T}=\mathrm{T}_{2}, \text { quality }=\mathrm{x}_{2}\right) \\
& \mathrm{h}_{2}=\operatorname{enthalpy}\left(\text { Steam }, \mathrm{T}=\mathrm{T}_{2}, \text { quality }=\mathrm{x}_{2}\right) \\
& \mathrm{s}_{2}=\text { entropy }\left(\text { Steam }, \mathrm{T}=\mathrm{T}_{2}, \text { quality }=\mathrm{x}_{2}\right) \\
& \mathrm{v}_{2}=\operatorname{volume}\left(\text { Steam }, \mathrm{T}=\mathrm{T}_{2}, \text { quality }=\mathrm{x}_{2}\right)
\end{aligned}
$$

The condenser water is supplied and returned at the temperatures and pressures associated with the ARTI specified conditions. The condenser water enters the condenser at $85^{\circ} \mathrm{F}$ and 1 atmosphere and leaves at $95^{\circ} \mathrm{F}$ and atmospheric pressure plus some pressure required to overcome friction in the piping system ( $\mathrm{p}_{\text {drop,piping }}$ ) and hydrostatic head associated with a cooling tower ( $\mathrm{p}_{\text {drop,tower }}$ ).

$$
\mathrm{T}_{3}=85^{\circ} \mathrm{F}
$$




$$
\mathrm{p}_{3}=\mathrm{p}_{\mathrm{atm}}
$$

The remaining state properties for the entering condenser water are determined.

$$
\begin{aligned}
& \mathrm{h}_{3}=\text { enthalpy }\left(\text { Steam, } \mathrm{T}=\mathrm{T}_{3}, \mathrm{p}=\mathrm{p}_{3}\right) \\
& \mathrm{s}_{3}=\operatorname{entropy}\left(\text { Steam, } \mathrm{T}=\mathrm{T}_{3}, \mathrm{p}=\mathrm{p}_{3}\right) \\
& \mathrm{v}_{3}=\operatorname{volume}\left(\text { Steam, } \mathrm{T}=\mathrm{T}_{3}, \mathrm{p}=\mathrm{p}_{3}\right)
\end{aligned}
$$

Upon entering the condenser, the condenser water is throttled from atmospheric pressure to the prevailing condensing pressure through a number of nozzles that distribute the water as a spray that wets the packing material. The nozzles are considered to be isenthalpic.

$$
\begin{aligned}
& \mathrm{h}_{4}=\mathrm{h}_{3} \\
& \mathrm{p}_{4}=\mathrm{p}_{2}
\end{aligned}
$$

The remaining state properties for the throttled condenser water are determined.

$$
\begin{aligned}
& \mathrm{T}_{4}=\text { temperature }\left(\text { Steam, } \mathrm{p}=\mathrm{p}_{4}, \mathrm{~h}=\mathrm{h}_{4}\right) \\
& \mathrm{s}_{4}=\operatorname{entropy}\left(\text { Steam, } \mathrm{p}=\mathrm{p}_{4}, \mathrm{~h}=\mathrm{h}_{4}\right) \\
& \mathrm{v}_{4}=\operatorname{volume}\left(\text { Steam, } \mathrm{p}=\mathrm{p}_{4}, \mathrm{~h}=\mathrm{h}_{4}\right)
\end{aligned}
$$

After flowing through the condenser and coming into intimate contact with the refrigerant vapor, the condenser water reaches an intermediate temperature $\left(T_{5}\right)$ that is just below the required $95^{\circ} \mathrm{F}$ exit temperature. The pressure is set equal to the exiting refrigerant pressure.

$$
\mathrm{p}_{5}=\mathrm{p}_{2}
$$

The exiting condenser water is returned at high pressure: atmospheric pressure plus the pressure rise required to overcome pressure losses in the piping and cooling tower. The enthalpy is determined using the isentropic pump efficiency ( $\left.\eta_{\text {pump }}\right)$.

$$
\left.\mathrm{h}_{6 \mathrm{~s}}=\text { enthalpy(Steam, } \mathrm{p}=\mathrm{p}_{6}, \mathrm{~s}=\mathrm{s}_{5}\right)
$$




$$
\eta_{\text {pump }}=\frac{\mathrm{h}_{6 \mathrm{~s}}-\mathrm{h}_{5}}{\mathrm{~h}_{6}-\mathrm{h}_{5}}
$$

where $\mathrm{h}_{6 \mathrm{~s}}$ is the isentropic pump exit enthalpy.

The pressure and enthalpy of the condenser water prior to entering the pump are used to determine the remainder of the state properties.

$$
\begin{aligned}
& \mathrm{T}_{5}=\text { temperature }\left(\text { Steam, } \mathrm{p}=\mathrm{p}_{5}, \mathrm{~h}=\mathrm{h}_{5}\right) \\
& \mathrm{s}_{5}=\operatorname{entropy}\left(\text { Steam, } \mathrm{p}=\mathrm{p}_{5}, \mathrm{~h}=\mathrm{h}_{5}\right) \\
& \mathrm{v}_{5}=\operatorname{volume}\left(\text { Steam, } \mathrm{p}=\mathrm{p}_{5}, \mathrm{~h}=\mathrm{h}_{5}\right)
\end{aligned}
$$

The condenser water exit conditions are set by the ARTI standard conditions.

$$
\begin{gathered}
\mathrm{T}_{6}=95^{\circ} \mathrm{F} \\
\mathrm{p}_{6}=\mathrm{p}_{\mathrm{atm}}+\mathrm{p}_{\text {drop,piping }}+\mathrm{p}_{\text {drop,tower }}
\end{gathered}
$$

The remaining state properties for the returning condenser water are determined using this temperature and pressure.

$$
\begin{aligned}
& \mathrm{h}_{6}=\operatorname{enthalpy}\left(\text { Steam, } \mathrm{T}=\mathrm{T}_{6}, \mathrm{p}=\mathrm{p}_{6}\right) \\
& \mathrm{s}_{6}=\operatorname{entropy}\left(\text { Steam, } \mathrm{T}=\mathrm{T}_{6}, \mathrm{p}=\mathrm{p}_{6}\right) \\
& \mathrm{v}_{6}=\operatorname{volume}\left(\text { Steam, } \mathrm{T}=\mathrm{T}_{6}, \mathrm{p}=\mathrm{p}_{6}\right)
\end{aligned}
$$

The condenser water mass flow rate is found using an overall energy balance.

$$
\dot{\mathrm{m}}_{\mathrm{cw}}=\dot{\mathrm{m}}_{\mathrm{ref}} \cdot \frac{\left(\mathrm{h}_{1}-\mathrm{h}_{2}\right)}{\left(\mathrm{h}_{5}-\mathrm{h}_{4}\right)}
$$

The effectiveness - NTU method is used to estimate the total required conductance (UA) of the direct-contact heat exchanger. The effectiveness is defined in terms of the actual and maximum possible temperature change that the fluid stream could see. 


$$
\varepsilon=\frac{\mathrm{T}_{1}-\mathrm{T}_{2}}{\mathrm{~T}_{1}-\mathrm{T}_{4}}
$$

The number of transfer units (NTU) is found using the relationship for a heat exchanger with a capacity ratio of zero (Incropera and DeWitt, 2002).

$$
\mathrm{NTU}=-\ln (1-\varepsilon)
$$

The required conductance is calculated using the definition of NTU for any heat exchanger configuration and the minimum capacitance rate.

$$
\mathrm{NTU}=\frac{\mathrm{UA}}{\dot{\mathrm{C}}_{\text {min }}}
$$

The minimum capacitance rate $\left(\dot{\mathrm{C}}_{\min }\right)$ is based on the water side.

$$
\dot{\mathrm{C}}_{\text {min }}=\dot{\mathrm{m}}_{\mathrm{cw}} \cdot \mathrm{C}_{\mathrm{p}, \mathrm{cw}}
$$

To relate the total conductance calculated above as well as the pressure loss specification to a physical size and heat exchanger geometry, it is necessary to specify the packing geometry in order to estimate the heat transfer coefficient and friction factor. The packing material is assumed to be similar to Brentwood Industries Model CF1200 fill media (Brentwood Industries, 2003). This media has a high specific surface area of $69 \mathrm{ft}^{2} / \mathrm{ft}^{3}$, which is consistent with the high performance requirements for this application. The packing material consists of triangular flow passages stacked in a cross-corrugated fashion. Therefore, the characteristic hydraulic diameter is considered to be the length of one side of the passage $\left(\mathrm{L}_{\text {side }}\right)$, which is approximately 0.5 in $(12 \mathrm{~mm})$. In the best-case scenario, $100 \%$ of the packing material will be wetted.

The heat transfer coefficient is estimated based on forced film condensation in a tube. When the inlet vapor Reynolds number is less than $35,000\left(\operatorname{Re}=\frac{\rho_{\mathrm{v}} \cdot \mathrm{v} \cdot \mathrm{D}_{\mathrm{h}}}{\mu_{\mathrm{v}}}\right)$, a 
heat transfer coefficient can be calculated based on the following parameters: the acceleration due to gravity $(\mathrm{g})$, the liquid density $\left(\rho_{\mathrm{l}}\right)$, the vapor density $\left(\rho_{\mathrm{v}}\right)$, the thermal conductivity of the liquid $\left(\mathrm{k}_{1}\right)$, a modified heat of vaporization $\left(\mathrm{h}_{\mathrm{fg}}^{\prime}\right)$, the viscosity of the liquid $\left(\mu_{1}\right)$, the saturation temperature of the liquid at the condenser pressure $\left(\mathrm{T}_{\text {sat }}\right)$, the temperature of the condenser water $\left(T_{s}\right)$, and the tube hydraulic diameter $\left(D_{h}\right)$.

$$
\begin{aligned}
& \rho_{\mathrm{l}}=\operatorname{density}\left(\operatorname{Steam}, \mathrm{T}=\mathrm{T}_{2}, \text { quality }=0\right) \\
& \rho_{\mathrm{v}}=\operatorname{density}\left(\text { Steam }, \mathrm{T}=\mathrm{T}_{2}, \text { quality }=1\right) \\
& \mathrm{k}_{\mathrm{l}}=\text { conductivity }\left(\text { Steam }, \mathrm{T}=\mathrm{T}_{2}, \text { quality }=0\right) \\
& \mathrm{h}_{\mathrm{fg}}^{\prime}+\frac{3}{8} \cdot \mathrm{C}_{\mathrm{p}, 1} \cdot\left(\mathrm{T}_{\text {sat }}-\mathrm{T}_{\mathrm{s}}\right) \\
& \mu_{1}=\text { viscosity }\left(\text { Steam, } \mathrm{T}=\mathrm{T}_{2}, \text { quality }=0\right) \\
& \mathrm{T}_{\text {sat }}=\text { temperature }\left(\text { Steam }, \mathrm{p}=\mathrm{p}_{2}, \text { quality }=0\right) \\
& \mathrm{T}_{\mathrm{s}}=\frac{\mathrm{T}_{\text {condenser water, }, \mathrm{in}}+\mathrm{T}_{\text {condenser water,out }}}{2} \\
& \mathrm{D}_{\mathrm{h}} \approx \mathrm{L}_{\text {side }}
\end{aligned}
$$

The heat transfer coefficient is calculated using Equation (4.213).

$$
\overline{\mathrm{h}}_{\mathrm{D}}=0.555 \cdot\left[\frac{\mathrm{g} \cdot \rho_{1} \cdot\left(\rho_{1}-\rho_{\mathrm{v}}\right) \cdot \mathrm{k}_{1}^{3} \cdot \mathrm{h}_{\mathrm{fg}}^{\prime}}{\mu_{1} \cdot\left(\mathrm{T}_{\mathrm{sat}}-\mathrm{T}_{\mathrm{s}}\right) \cdot \mathrm{D}_{\mathrm{h}}}\right]^{1 / 4}
$$

The Reynolds number for the refrigerant vapor passing through the packing material is $\sim 329$, thus the preceding heat transfer correlation holds.

The specific surface area is used to determine the required heat exchanger volume $\left(\mathrm{V}_{\mathrm{HX}}\right)$.

$$
\mathrm{a}_{\mathrm{s}}=\eta_{\text {pack }} \cdot \frac{\mathrm{A}_{\mathrm{HX}}}{\mathrm{V}_{\mathrm{HX}}}
$$

where $a_{s}$ is the specific surface area of the fill media and $\eta_{\text {pack }}$ is the percentage of wetted surface of the total packing surface area (for this study, $\eta_{\text {pack }}=100 \%$ ). 
The pressure drop for the packing is determined based on experimental data conducted at the UW-Madison using air passing through the packing material wetted with water (Reindl, 1996). Contraction and exit losses are ignored and the data is used to determine an appropriate friction factor.

$$
\frac{\Delta \mathrm{p}_{\text {air }}}{\mathrm{L}_{\mathrm{HX}}}=\frac{\mathrm{f}}{\mathrm{D}_{\mathrm{h}}} \cdot \frac{1}{2} \cdot \rho_{\text {air }} \cdot \mathrm{v}_{\text {air }}^{2}
$$

Using the data and test conditions (pressure drop, packing length, air density, and velocity), the quantity $\frac{f}{D_{h}}$ is determined; $\frac{f}{D_{h}}$ is renamed ' $k$ ' in the following equation.

For refrigerant vapor moving through this same packing material, the k-value calculated above from the air data is assumed to be approximately independent of the Reynolds number. This k-value is used to estimate the pressure drop associated with the condensing refrigerant vapor flowing through the direct-contact condenser packing media.

$$
\frac{\Delta \mathrm{p}_{\text {vapor }}}{\mathrm{L}_{\mathrm{HX}}}=\mathrm{k} \cdot \frac{1}{2} \cdot \rho_{\mathrm{v}} \cdot\left(\frac{\mathrm{v}}{2}\right)^{2}
$$

Note that half the inlet refrigerant vapor velocity (v) is used in the preceding pressure drop equation as this represents the average velocity in the condenser.

$$
\mathrm{v}=\frac{2 \cdot \dot{\mathrm{m}}_{\mathrm{ref}}}{\rho_{\mathrm{v}} \cdot \pi \cdot \mathrm{D}_{\mathrm{HX}}^{2}}
$$

where $\mathrm{D}_{\mathrm{HX}}$ is the diameter of the heat exchanger.

The pressure loss cannot be neglected when sizing the direct-contact heat exchanger for this application. Based on cycle performance requirements, the maximum allowable pressure drop is set at $2 \%$ of the total refrigerant vapor condensing pressure. 
Combining the above equations, heat exchanger aspect ratio $\left(\mathrm{L}_{\mathrm{HX}} / \mathrm{D}_{\mathrm{HX}}\right)$ is to be set based only on the pressure loss; the smaller the allowable pressure loss, the smaller the aspect ratio (i.e., the heat exchanger becomes more "pancake" shaped). The required heat transfer area is known from the total conductance and the heat transfer coefficient.

$$
\mathrm{UA}=\mathrm{h} \cdot \mathrm{A}_{\mathrm{HX}}
$$

The total heat exchanger volume is based on the heat transfer area and the specific area of the packing. The volume together with the aspect ratio allows the diameter (and therefore length) of the heat exchanger to be determined.

$$
\mathrm{V}_{\mathrm{HX}}=\frac{\pi}{4} \cdot \mathrm{D}_{\mathrm{HX}}^{2} \cdot \mathrm{L}_{\mathrm{HX}}
$$

\subsubsection{Size}

The direct-contact condenser is significantly smaller than a comparable indirectcontact condenser for the same application. The dimensions of the direct-contact condenser are compared to that of the indirect-contact condenser required for the same operating conditions for water-as-a-refrigerant and R-134a in Table 24.

Table 24 Direct-contact condenser dimensions.

\begin{tabular}{c|c|c|c} 
Dimension & $\begin{array}{c}\text { Water Direct } \\
\text { Contact Condenser }\end{array}$ & $\begin{array}{c}\text { Water Indirect } \\
\text { Contact Condenser }\end{array}$ & $\begin{array}{c}\text { R-134a Indirect- } \\
\text { contact Condenser }\end{array}$ \\
\hline $\mathrm{D}_{\mathrm{HX}}, \mathrm{ft}$ & 8.2 & 5.5 & 3.0 \\
$\mathrm{~L}_{\mathrm{HX}}, \mathrm{ft}$ & 0.96 & 24 & 13.0 \\
$\mathrm{~V}_{\mathrm{HX}}, \mathrm{ft}^{3}$ & 50.7 & 570 & 91.9
\end{tabular}

Table 24 shows that the direct-contact condenser is smaller than its indirectcontact counterpart by a factor of approximately 10 for the water-cycle and is smaller by a factor of approximately 2 than the indirect condenser for the R-134a cycle. Even disregarding this savings in physical size, the direct-contact heat exchanger should be 
substantially lower cost based on its relatively simple construction, ease of headering, lack of pressure boundaries, and lower material cost.

\subsubsection{Cost}

The cost of a direct-contact heat exchanger is related to the volume enclosed by the shell and the amount of packing material present inside the shell. Because the packing material is directly proportional to the shell volume, the cost of a direct-contact condenser is entirely dependent on the shell volume. The equation developed for the cost of the shell for an indirect-contact heat exchanger is used without modification.

$$
\text { Shell Cost }=\left(\frac{\$ 25}{\mathrm{ft}^{3}}\right) \cdot \frac{\pi}{4} \cdot \mathrm{D}_{\mathrm{HX}, \mathrm{ft}}^{2} \cdot \mathrm{L}_{\mathrm{HX}, \mathrm{ft}}
$$

The packing material cost is calculated on a volume basis (Brentwood Industries, 2003).

$$
\text { Packing Cost }=\left(\frac{\$ 5.40}{\mathrm{ft}^{3}}\right) \cdot \frac{\pi}{4} \cdot \mathrm{D}_{\mathrm{HX}, \mathrm{ft}}^{2} \cdot \mathrm{L}_{\mathrm{HX}, \mathrm{ft}}
$$

The overall cost of a direct-contact condenser is compared to the indirect-contact condenser both for water as a refrigerant in Table 25 .

Table 25 Price comparison between direct and indirect condensers.

\subsubsection{Direct-Contact Evaporator Model}

The direct-contact evaporator model is similar to the direct-contact condenser model for water vapor as a refrigerant. The details of this model are described in this section. 


\subsubsection{Performance}

In a direct-contact heat exchanger, the working fluid used as a refrigerant is identical to the secondary distribution working fluid. For heat exchanger calculations, the water acting as a primary refrigerant will be referred to as the "refrigerant" and the water acting as a secondary refrigerant will be referred to as the "chilled water". Table 26 shows the locations of all the states in the direct-contact evaporator as illustrated in the schematic shown in Figure 47.

Table 26 Indexing for states in direct-contact evaporator.

\begin{tabular}{c|l} 
State & Location \\
\hline 1 & Refrigerant inlet \\
2 & Refrigerant exit \\
3 & Chilled water supply \\
4 & Chilled water after throttle \\
5 & Chilled water before pump \\
6 & Chilled water return
\end{tabular}




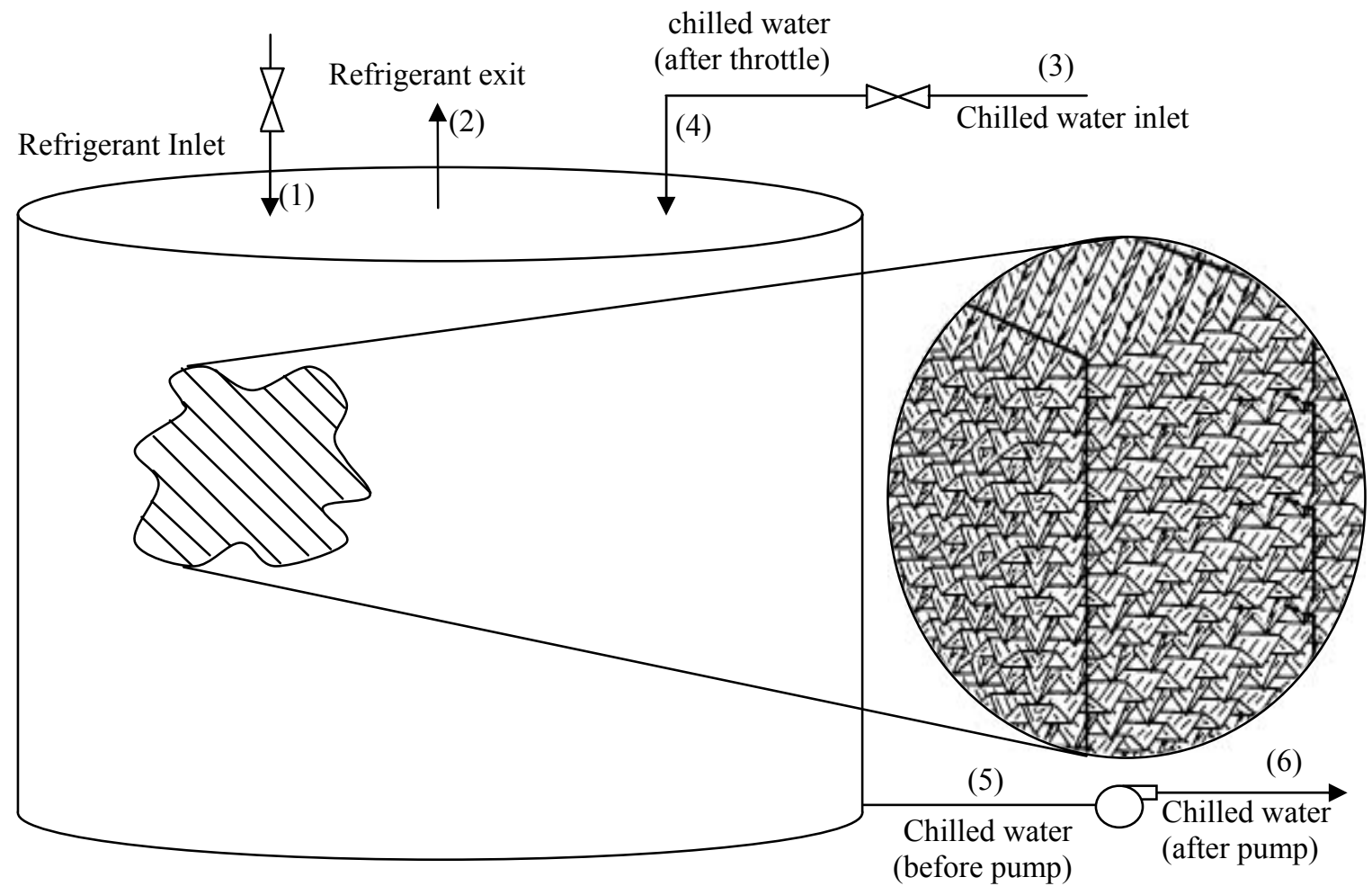

Figure 47 Direct-contact evaporator schematic.

The refrigerant side states are calculated using variables passed from both the main program and the condenser. The incoming refrigerant is assumed to undergo an isenthalpic throttling process. Thus, the enthalpy is passed from the condenser and used to determine the inlet quality based in the evaporator pressure.

$$
\begin{aligned}
& \mathrm{h}_{1}=\mathrm{h}_{\text {cond,out }} \\
& \mathrm{p}_{1}=\mathrm{p}_{2}+\Delta \mathrm{p}
\end{aligned}
$$

where $\Delta \mathrm{p}$ is the refrigerant pressure drop through the evaporator.

The remainder of the thermodynamic state properties is determined based on this pressure and enthalpy. 


$$
\begin{aligned}
& \mathrm{T}_{1}=\text { temperature }\left(\text { Steam, } \mathrm{p}=\mathrm{p}_{1}, \mathrm{~h}=\mathrm{h}_{1}\right) \\
& \mathrm{s}_{1}=\operatorname{entropy}\left(\text { Steam, } \mathrm{p}=\mathrm{p}_{1}, \mathrm{~h}=\mathrm{h}_{1}\right) \\
& \mathrm{v}_{1}=\operatorname{volume}\left(\text { Steam, } \mathrm{p}=\mathrm{p}_{1}, \mathrm{~h}=\mathrm{h}_{1}\right) \\
& \mathrm{x}_{1}=\text { quality }\left(\text { Steam, } \mathrm{p}=\mathrm{p}_{1}, \mathrm{~h}=\mathrm{h}_{1}\right)
\end{aligned}
$$

The refrigerant exit is assumed to be a saturated vapor with no superheat.

$$
\mathrm{x}_{2}=1
$$

The saturated evaporator temperature is determined based on a specified pinch point temperature difference $\left(\Delta \mathrm{T}_{\text {evap }}\right)$.

$$
\mathrm{T}_{2}=\mathrm{T}_{\text {chillwater,out }}-\Delta \mathrm{T}_{\text {evap }}
$$

The remainder of the state properties is based on temperature and quality.

$$
\begin{aligned}
& \mathrm{p}_{2}=\text { pressure }\left(\text { Steam }, \mathrm{T}=\mathrm{T}_{2}, \text { quality }=0\right) \\
& \mathrm{h}_{2}=\operatorname{enthalpy}\left(\text { Steam }, \mathrm{T}=\mathrm{T}_{2}, \text { quality }=0\right) \\
& \mathrm{s}_{2}=\operatorname{entropy}\left(\text { Steam }, \mathrm{T}=\mathrm{T}_{2}, \text { quality }=0\right) \\
& \mathrm{v}_{2}=\operatorname{volume}\left(\text { Steam }, \mathrm{T}=\mathrm{T}_{2}, \text { quality }=0\right)
\end{aligned}
$$

The chilled water inlet state is determined by the pressure and the temperature specified by ARTI condition.

$$
\begin{aligned}
& \mathrm{p}_{3}=1 \mathrm{~atm} \\
& \mathrm{~T}_{3}=54^{\circ} \mathrm{F}
\end{aligned}
$$

The remainder of the state properties for the incoming chilled water is determined based on temperature and pressure.

$$
\begin{aligned}
& \mathrm{h}_{3}=\operatorname{enthalpy}\left(\text { Steam, } \mathrm{T}=\mathrm{T}_{3}, \mathrm{p}=\mathrm{p}_{3}\right) \\
& \mathrm{s}_{3}=\operatorname{entropy}\left(\text { Steam, } \mathrm{T}=\mathrm{T}_{3}, \mathrm{p}=\mathrm{p}_{3}\right) \\
& \mathrm{v}_{3}=\operatorname{volume}\left(\text { Steam, } \mathrm{T}=\mathrm{T}_{3}, \mathrm{p}=\mathrm{p}_{3}\right)
\end{aligned}
$$

Upon entering the direct-contact evaporator, the chilled water must be throttled to the evaporator pressure. The throttling process is assumed to by isenthalpic. The chilled 
water properties downstream of the throttling valve are determined using the pressure and the enthalpy.

$$
\begin{aligned}
& \mathrm{p}_{4}=\mathrm{p}_{1} \\
& \mathrm{~h}_{4}=\mathrm{h}_{3}
\end{aligned}
$$

The remainder of the state properties is determined based on pressure and enthalpy.

$$
\begin{aligned}
& \mathrm{T}_{4}=\text { temperature }\left(\text { Steam, } \mathrm{p}=\mathrm{p}_{4}, \mathrm{~h}=\mathrm{h}_{4}\right) \\
& \mathrm{s}_{4}=\operatorname{entropy}\left(\text { Steam, } \mathrm{p}=\mathrm{p}_{4}, \mathrm{~h}=\mathrm{h}_{4}\right) \\
& \mathrm{v}_{4}=\operatorname{volume}\left(\text { Steam, } \mathrm{p}=\mathrm{p}_{4}, \mathrm{~h}=\mathrm{h}_{4}\right)
\end{aligned}
$$

Leaving the evaporator, the state of the chilled water prior to the pump is determined by the pump efficiency and the pressure drop through the evaporator.

$$
\begin{gathered}
\mathrm{p}_{5}=\mathrm{p}_{2}-\Delta \mathrm{p}_{\text {evap }} \\
\eta_{\text {pump }}=\frac{\mathrm{h}_{6 \mathrm{~s}}-\mathrm{h}_{5}}{\mathrm{~h}_{6}-\mathrm{h}_{5}}
\end{gathered}
$$

where $\mathrm{h}_{6 \mathrm{~s}}$ is the enthalpy corresponding to an isentropic increase in pressure to the water distribution system supply pressure.

The remainder of the state properties is calculated based on pressure and enthalpy.

$$
\begin{aligned}
& \mathrm{T}_{5}=\text { temperature }\left(\text { Steam, } \mathrm{p}=\mathrm{p}_{5}, \mathrm{~h}=\mathrm{h}_{5}\right) \\
& \mathrm{s}_{5}=\operatorname{entropy}\left(\text { Steam, } \mathrm{p}=\mathrm{p}_{5}, \mathrm{~h}=\mathrm{h}_{5}\right) \\
& \mathrm{v}_{5}=\operatorname{volume}\left(\text { Steam, } \mathrm{p}=\mathrm{p}_{5}, \mathrm{~h}=\mathrm{h}_{5}\right)
\end{aligned}
$$

The chilled water return properties are known from the ARTI specified conditions.

$$
\begin{aligned}
& \mathrm{p}_{6}=1 \mathrm{~atm} \\
& \mathrm{~T}_{6}=44^{\circ} \mathrm{F}
\end{aligned}
$$

The remainder of the state properties is determined by the temperature and pressure. 


$$
\begin{aligned}
& \mathrm{h}_{6}=\text { enthalpy }\left(\text { Steam, } \mathrm{T}=\mathrm{T}_{6}, \mathrm{p}=\mathrm{p}_{6}\right) \\
& \mathrm{s}_{6}=\operatorname{entropy}\left(\text { Steam, } \mathrm{T}=\mathrm{T}_{6}, \mathrm{p}=\mathrm{p}_{6}\right) \\
& \mathrm{v}_{6}=\operatorname{volume}\left(\text { Steam, } \mathrm{T}=\mathrm{T}_{6}, \mathrm{p}=\mathrm{p}_{6}\right) \\
& \mathrm{h}_{6 \mathrm{~s}}=\text { enthalpy }\left(\text { Steam, } \mathrm{p}=\mathrm{p}_{6}, \mathrm{~s}=\mathrm{s}_{5}\right)
\end{aligned}
$$

With the properties for all direct-contact evaporator state points determined, an energy balance is used to determine the required mass flow rates of both the refrigerant and the chilled water. ARTI specifies that the refrigeration system must handle a 1000ton refrigeration load (CoolCapacity $=1000$ tons $)$

$$
\begin{gathered}
\text { CoolCapacity }=\dot{\mathrm{m}}_{\mathrm{ref}} \cdot\left(\mathrm{h}_{2}-\mathrm{h}_{1}\right) \\
\dot{\mathrm{m}}_{\mathrm{chw}}=\frac{\text { CoolCapacity }}{\mathrm{h}_{4}-\mathrm{h}_{6}}
\end{gathered}
$$

The $\varepsilon$-NTU method is used to relate the heat transfer to the heat exchanger geometry. The heat exchanger effectiveness $(\varepsilon)$ is a measure of the maximum theoretical heat transfer relative to the actual heat transferred by the heat exchanger.

$$
\varepsilon=\frac{\mathrm{T}_{3}-\mathrm{T}_{6}}{\mathrm{~T}_{3}-\mathrm{T}_{2}}
$$

For a heat exchanger with one stream undergoing a phase change, the number of transfer units (NTU) is calculated in terms of the heat exchanger effectiveness according to:

$$
\mathrm{NTU}=-\ln (1-\varepsilon)
$$

Using the definition of the NTU, the heat exchanger effectiveness is related to the heat exchanger conductance (UA).

$$
\mathrm{NTU}=\frac{\mathrm{UA}}{\dot{\mathrm{C}}_{\text {min }}}
$$


The heat transfer coefficient is found using the same method described for the direct-contact condenser.

\subsubsection{Size}

The size of the direct-contact evaporator is much smaller than the indirect-contact evaporator required for this application. Table 27 is a comparison between the directand indirect-contact evaporators for both refrigerants.

Table 27 Evaporator dimensional comparison of water direct/indirect and R-134a cycles.

\begin{tabular}{c|c|c|c} 
Dimension & $\begin{array}{c}\text { Water Direct } \\
\text { Contact Evaporator }\end{array}$ & $\begin{array}{c}\text { Water Indirect- } \\
\text { contact Evaporator }\end{array}$ & $\begin{array}{c}\text { R-134a Indirect- } \\
\text { contact Evaporator }\end{array}$ \\
\hline $\mathrm{D}_{\mathrm{HX}}, \mathrm{ft}$ & 9.53 & 5.1 & 3.5 \\
$\mathrm{~L}_{\mathrm{HX}}, \mathrm{ft}$ & .93 & 20.5 & 14 \\
$\mathrm{~V}_{\mathrm{HX}}, \mathrm{ft}^{3}$ & 66.3 & 430 & 134.7
\end{tabular}

The size disparity will show up in the next section as a significant savings over the indirect-contact evaporator for both water as a refrigerant and for R-134a as a refrigerant.

\subsubsection{Cost}

The cost of a direct-contact heat exchanger is related to the volume enclosed by the shell and the amount of packing material present inside the shell. Because the amount of packing material is directly proportional to the shell volume, the cost of a directcontact evaporator is entirely dependent on the shell volume. The equation developed for the cost of the shell for an indirect-contact heat exchanger is used without modification.

$$
\text { Shell Cost }=\left(\frac{\$ 22.20}{\mathrm{ft}^{3}}\right) \cdot \frac{\pi}{4} \cdot \mathrm{D}_{\mathrm{HX}, \mathrm{ft}}^{2} \cdot \mathrm{L}_{\mathrm{HX}, \mathrm{ft}}
$$

The packing material cost is calculated on a volume basis (Brentwood Industries, 2003). 


$$
\text { Packing Cost }=\left(\frac{\$ 5.40}{\mathrm{ft}^{3}}\right) \cdot \frac{\pi}{4} \cdot \mathrm{D}_{\mathrm{HX}, \mathrm{ft}}^{2} \cdot \mathrm{L}_{\mathrm{HX}, \mathrm{ft}}
$$

The overall cost of a direct-contact evaporator is compared to the indirect-contact evaporator in Table 28.

Table 28 Price comparison between direct and indirect evaporators.

\begin{tabular}{c|c} 
Direct-contact Evaporator & Indirect-contact Evaporator \\
\hline$\$ 3,100$ & $\$ 37,600$
\end{tabular}

\subsubsection{Intercooler}

Flash intercooling was chosen for the two-stage water as refrigerant cycle. Flash intercooling implies a direct-contact heat exchanger. The intercooling process is nearly identical to the process associated with the direct-contact condenser or evaporator. However, the intercooler is intended only for desuperheating, whereas the condenser is dedicated to liquefaction. Because the vapor velocity is so high through the intercooler, it is advantageous to use spray nozzles to affect the cooling. The intercooler model determines the appropriate intercooler size to desuperheat the refrigerant exiting the lowpressure compressor as well as the associated pressure drop and the required liquid flow rate given an approach temperature specification (here approach temperature difference is related to how close the vapor comes to the vapor dome). The intercooler is assumed to be a smooth tube that has a diameter equal to the shroud of the high pressure compressor inlet. At the inlet to the intercooler is a bank of spray nozzles that will atomize the liquid water. The atomization process is assumed to be isenthalpic.

Spray nozzles are modeled rather than packing material, because the required heat transfer rate is much lower than in either the evaporator or the condenser as shown in Table 29. If an intercooler with packing material was chosen, the result would be a very small required surface area, the pancake shaped aspect ratio would be even more 
pronounced since the packing material has a higher pressure drop per unit length of vapor travel.

Table 29 Comparison of sizes of direct-contact heat exchangers (packing material used).

\begin{tabular}{r|c|c|c} 
& Evaporator & Condenser & Intercooler \\
\hline $\mathrm{L}_{\mathrm{HX}}$, in & 11.2 & 11.5 & 1.4 \\
$\mathrm{D}_{\mathrm{HX}}, \mathrm{ft}$ & 9.53 & 8.2 & 3.1 \\
$\mathrm{~A}_{\mathrm{HX}}, \mathrm{ft}^{2}$ & 4,574 & 3,498 & 59.4 \\
Heat Transfer, Btu/min & 200,000 & 233,000 & 13,000
\end{tabular}

The following sections are descriptions of the performance, size and cost of the intercooler model.

\subsubsection{Performance}

The performance of the intercooler is more important than its size, because of its inherently low capital cost relative to the other water cycle components. The intercooler size is approximately one order of magnitude smaller than the other heat exchangers. When compared with the cost of these other heat exchangers, the intercooler cost is negligible. For this reason, the size and cost of the intercooler are neglected in the economic analysis and the performance is set as a design parameter. Again, this is conservative as it slightly underestimates the capital cost of the water cycle.

Pressure drop is neglected in the intercooler; this assumption is justified because the pressure drop in a smooth tube with a diameter set equal to the compressor inlet shroud is minimal. The pressure drop per foot length of tube can be calculated.

$$
\frac{\Delta \mathrm{p}}{\mathrm{L}_{\text {int }}}=\frac{\mathrm{f}}{\mathrm{D}_{\text {int }}} \cdot \rho_{\mathrm{v}} \cdot \frac{\mathrm{v}^{2}}{2}
$$

where $f$ is the friction factor for the given tube and Reynolds number, $L_{\text {int }}, D_{\text {int }}$ are the intercooler length and diameter, $\rho_{\mathrm{v}}$ is the vapor density, and $\mathrm{v}$ is the vapor velocity. 
The pressure drop per foot $\left(\frac{\Delta \mathrm{p}}{\mathrm{L}_{\text {int }}} \mathrm{psi} / \mathrm{ft}\right)$ is calculated to be less than $1 \mathrm{e}-4 \mathrm{psi} / \mathrm{ft}$. Assuming that the intercooler length will be on the order of 5 feet, this pressure drop can be neglected in the performance calculations.

\subsubsection{Pump Model}

The pumping requirements for the water as a refrigerant system vary depending on the cycle configuration (i.e. indirect-contact heat exchangers v. direct-contact heat exchangers). The pumping requirements for the water-cycle evaporator are nearly identical to that for the R-134a cycle, because the water flow rates are identical and the calculation of the pressure drops are similar. The pumping requirement for the watercycle condenser can differ from the R-134a cycle due to differences in the cycle COP. If direct-contact heat exchange is selected for the cycle, an added pumping load is associated with the need to re-pressurize the condenser water and the chilled water to atmospheric pressure.

Refer to Section 4.1.4 for a description of pressure loss in a typical building piping system for a given cooling load. The pumping load associated with a water cycle using indirect-contact heat exchangers is estimated using a process that is identical to that used for the R-134a cycle. However, the added pump head requirement due to the directcontact heat exchangers is significant due to the high vacuum in the water-cycle. Because the water cycle operates under nearly vacuum conditions, the direct condenser pressure drop can be approximated by a drop equal to atmospheric pressure (14.7 psia). Thus, the overall pressure that the water pump must supply includes the pressure drop 
through the piping, the throttle, the heat exchanger, and for the condenser, the cooling tower.

\subsubsection{Performance}

This section provides a description of the pumps that are required to maintain adequate flow through the water-cycle. The pumping load on the cycle can be broken down into sub-categories: the pressure drop through the heat exchanger (tubing or packing), the pressure drop through the throttle, the pressure drop through piping, and through the cooling tower. Table 30 is a comparison of the condenser water and the chilled water pumping requirement for the direct-contact and indirect heat exchanger configurations.

Table 30 Comparison of pumping requirements for various heat exchanger configurations.

\begin{tabular}{l|c|c|c|c} 
& \multicolumn{2}{|c|}{$\begin{array}{c}\text { Indirect Heat } \\
\text { Exchangers }\end{array}$} & \multicolumn{2}{c}{$\begin{array}{c}\text { Direct-contact Heat } \\
\text { Exchangers }\end{array}$} \\
\hline & $\begin{array}{c}\text { Condenser } \\
\text { Water }\end{array}$ & $\begin{array}{c}\text { Chilled } \\
\text { Water }\end{array}$ & $\begin{array}{c}\text { Condenser } \\
\text { Water }\end{array}$ & $\begin{array}{c}\text { Chilled } \\
\text { Water }\end{array}$ \\
\hline Head Loss & 0.10 & 0.0 & 0.0 & 0.0 \\
Heat Exchanger, $\mathrm{ft}$ & $\mathrm{N} / \mathrm{A}$ & $\mathrm{N} / \mathrm{A}$ & 34.04 & 33.9 \\
Piping, $\mathrm{ft}$ & 5.8 & 81.7 & 5.8 & 81.7 \\
Cooling Tower, $\mathrm{ft}$ & 61.4 & $\mathrm{~N} / \mathrm{A}$ & 61.4 & $\mathrm{~N} / \mathrm{A}$ \\
\hline \hline Total Head, ft & 67.3 & 81.7 & 101.2 & 115.6 \\
\hline Vol. Flow Rate, gpm & 2804 & 2384 & 2804 & 2384
\end{tabular}

For the indirect heat exchanger configuration, the system model assumes that the condenser water pump has an isentropic efficiency of $82.3 \%$ based on a Bell and Gossett Model $15108 G$ pump. Because the condenser water has a higher volumetric flow rate than the chilled water, there is a larger pump used to handle the condenser water loop. The model assumes that the chilled water pump has an isentropic efficiency of $67.9 \%$ 
based on a Bell and Gossett Model 1510 6G pump (Bell and Gossett Pump Selection (online), 2003).

For the direct-contact heat exchanger configuration, the system model assumes that the condenser water pump has an isentropic efficiency of $85.8 \%$ based on a Bell and Gossett Model $15108 G$ pump. The model assumes that the chilled water pump has an isentropic efficiency of 78.7\% based on the Bell and Gossett Model 15106 pump.

Figures 48 through 51 are pump curves that indicate the individual pump requirements listed in Table 30 (the dot on each figure represents the nominal pump operating condition).

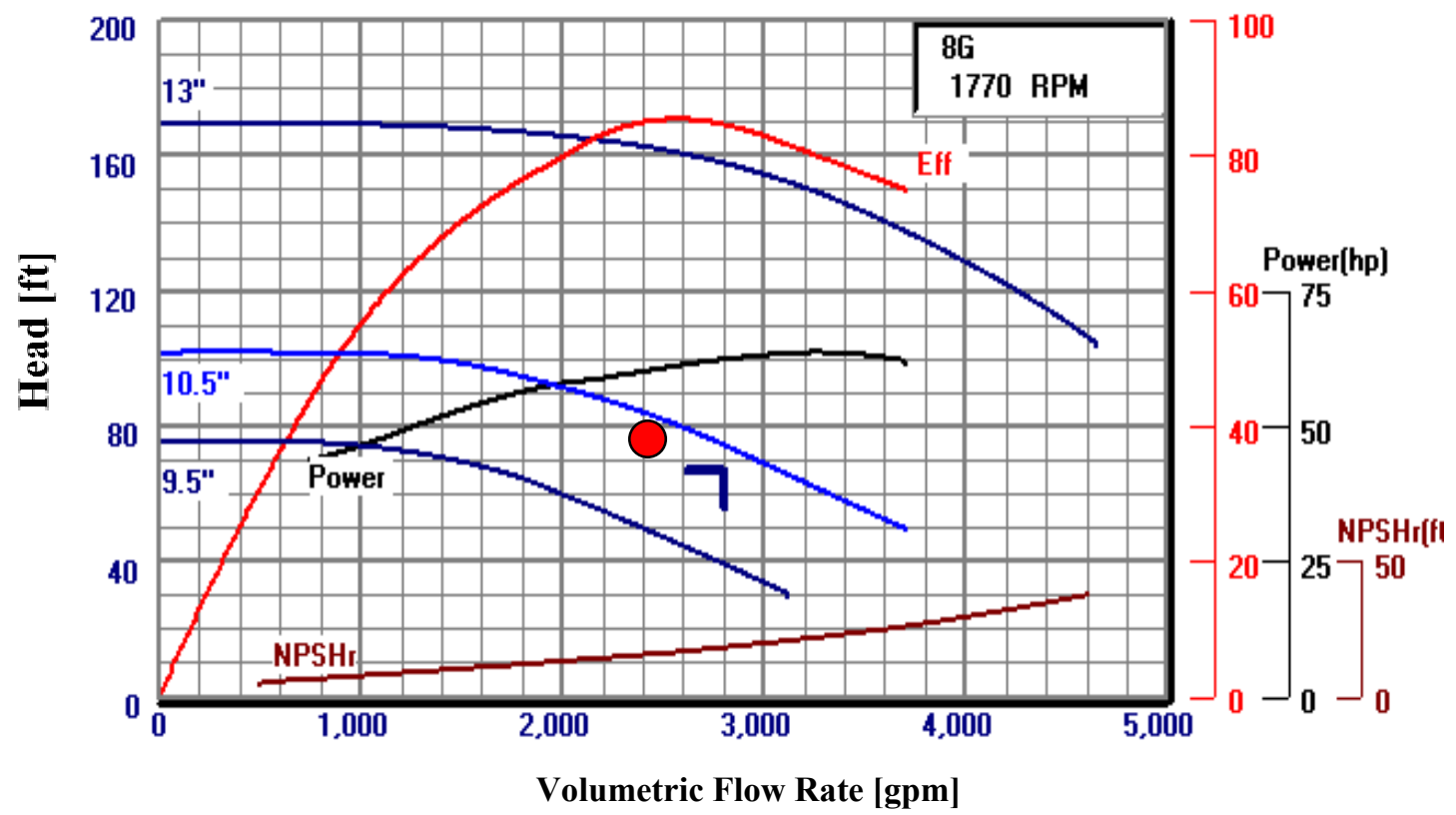

Figure 48 Operating conditions for condenser water pump for indirect-contact HX on the pump curve for Model 1510 8G (courtesy of Bell and Gossett, 2003). 


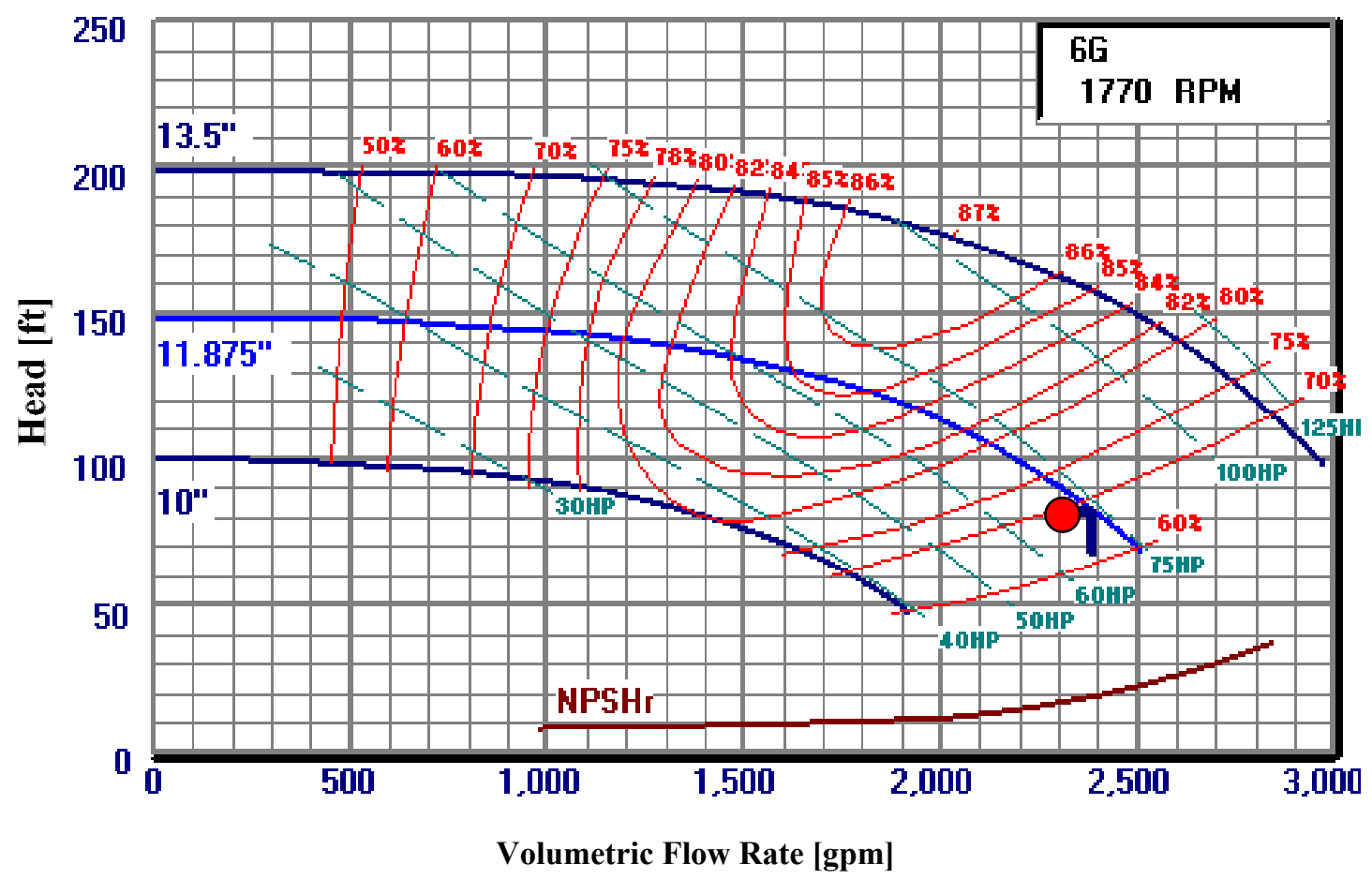

Figure 49 Operating conditions for chilled water pump for indirect-contact HX on the pump curve for Model 1510 6G (courtesy of Bell and Gossett, 2003).

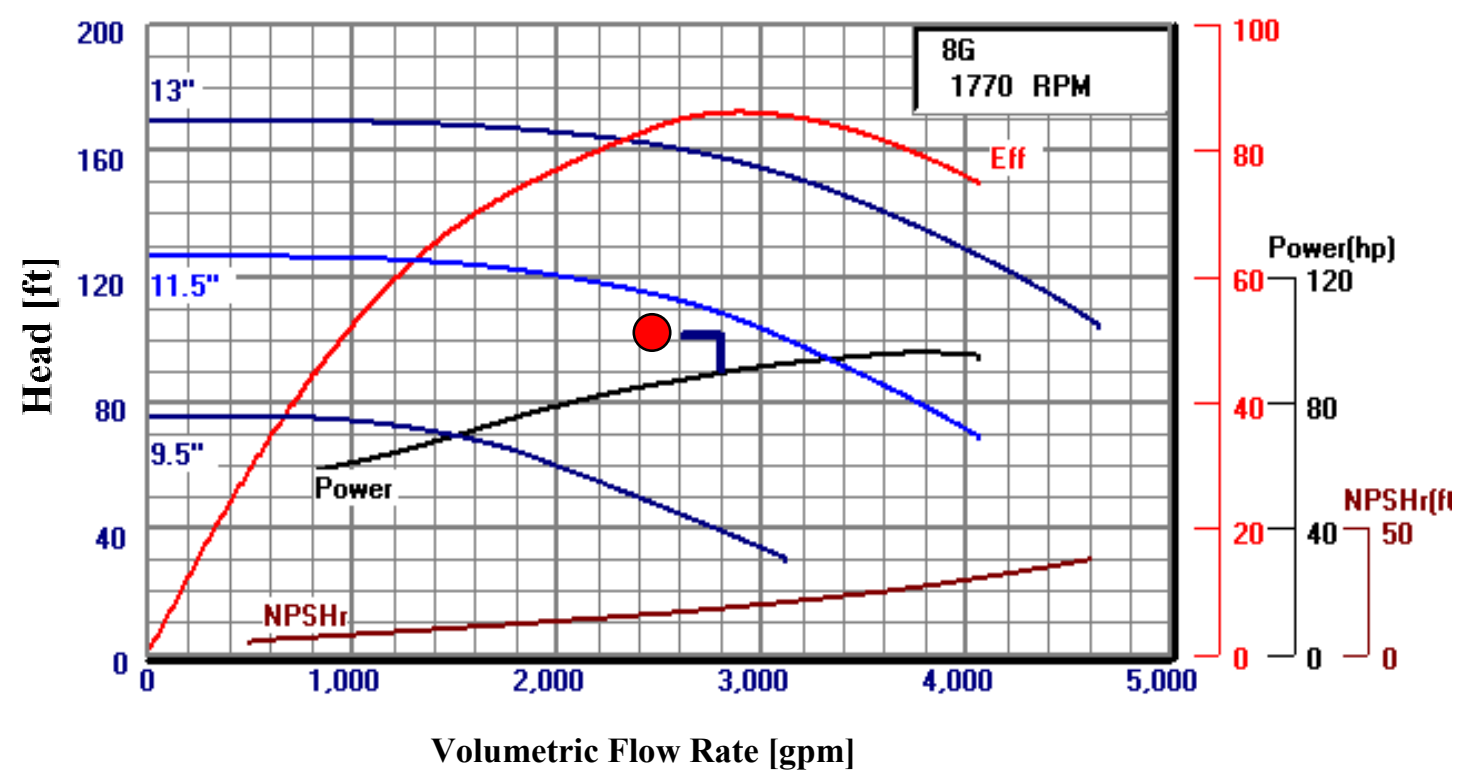

Figure 50 Operating conditions for condenser water pump for direct-contact HX on the pump curve for Model $15108 G$ (courtesy of Bell and Gossett, 2003). 


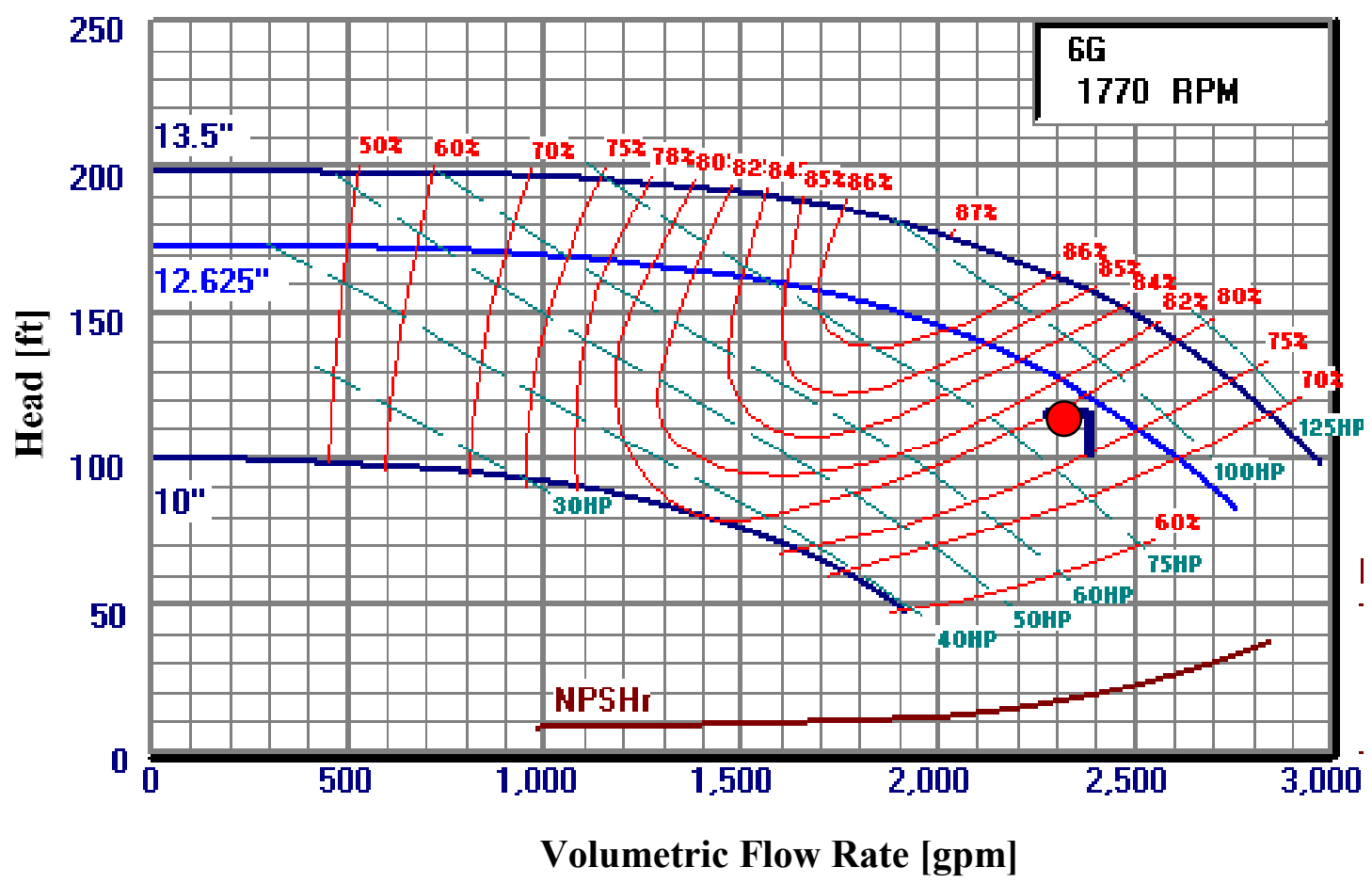

Figure 51 Operating condition for chilled water pump for direct-contact HX on the pump curve for Model 1510 6G (courtesy of Bell and Gossett, 2003).

\subsubsection{Cost}

There are two components to the cost of the water pumping systems: the cost of the pumps, and the cost of the energy required to run the pumps. There are two pumps required for the system, the chilled water pump and the condenser water pump. The chilled water pump, a Bell and Gossett model 1510 6G, costs $\$ 5950$. The condenser water pump, a Bell and Gossett model $15108 \mathrm{G}$, costs $\$ 6850$. The condenser water pump costs more than the chilled water pump due to its larger required flow rate and head. The energy cost of running the pumps is calculated for each cycle configuration in Chapter 5.

\subsubsection{Purging System}

When considering a system that utilizes a direct-contact heat exchanger, purging noncondensables becomes a significant issue. The water entering the direct-contact heat exchanger will contain some air that will escape upon expansion to a low pressure. Air 
significantly degrades heat exchanger performance and places an additional load on the compressors. The amount of air depends on many parameters related to the specifics of the overall system. In a direct-contact condenser, the water may flow through a cooling tower where the concentration of air absorbed will increase. In other systems, the amount of air in the water may be dependent on the hermeticity of the system. However, in any system a substantial amount of air will have to be removed upon startup.

The specific design of a purging system is beyond the scope of this project. The objective of this section is to bound the cycle penalty associated with purging in order to point out its importance. It is important to note that the economic analyses presented in Chapter 5 do not include any estimate of the purging cost due to the large uncertainty in the actual purge requirements. Therefore, these analyses are conservative relative to the cost of any system using a direct-contact heat exchanger. The following section is a description of the purging requirements and related hardware.

\subsubsection{Performance}

The water cycle economic analysis concentrates in large part on the benefit associated with a direct-contact heat exchanger relative to an indirect shell-and-tube heat exchanger. The performance of a direct-contact heat exchanger is inherently better than an indirect heat exchanger due to the lower achievable pinch-point temperature differences. The direct-contact heat exchanger is also less costly due to its relatively simpler construction and smaller size.

However, when either the condenser water or chilled water supplied to a directcontact heat exchanger are expanded to the condenser or evaporator pressures, the solubility of air in the water decreases and therefore noncondensables in the water will be 
released. Any direct-contact condenser system in which liquid phase water is brought into intimate contact with air will absorb noncondensables. Air released from the water upon its expansion into the direct-contact evaporator will tend to build up in the refrigeration system; less air is removed in the liquid water pumped out of the heat exchanger than is brought into the system by the chilled water because the solubility of air in water is proportional to pressure. The presence of significant amounts of air in the system will degrade the heat transfer capability of the condenser and reduce the capacity of the compressor.

One possibility for preventing this accumulation of air is the use of a secondary loop (e.g., a heat exchange circuit that thermally connects the condenser water with the cooling tower). The added pumps and heat exchangers associated with this loop will result in a substantially higher system cost.

In order to quantify the purging costs, it is necessary to determine the amount of air that is dissolved in water at the condenser water supply temperature. Figure 52 shows the variation of nitrogen solubility in water as a function of both temperature and pressure. Here it is assumed that nitrogen solubility in water is similar to the solubility of air.

With any water-as-refrigerant system (direct- or indirect-contact heat exchangers), there will tend to be water leakage through seals and joints. Although this leakage rate is difficult to quantify, it will inevitably create the need for a purging system. This system would be significantly smaller for the cycle with indirect-contact heat exchangers however for the reasons listed above. 
At start-up, the initial condenser or chilled water charge will be saturated with air; the extra air at start-up places a higher load on the compressor, making it more difficult to reach steady state. This results in a penalty on the COP which is the departure from a theoretical COP predicted without accounting for air infiltration. The air purging system will remove some water vapor along with air. This water must be made up somewhere in the system and this adds to the load on the purge system. 


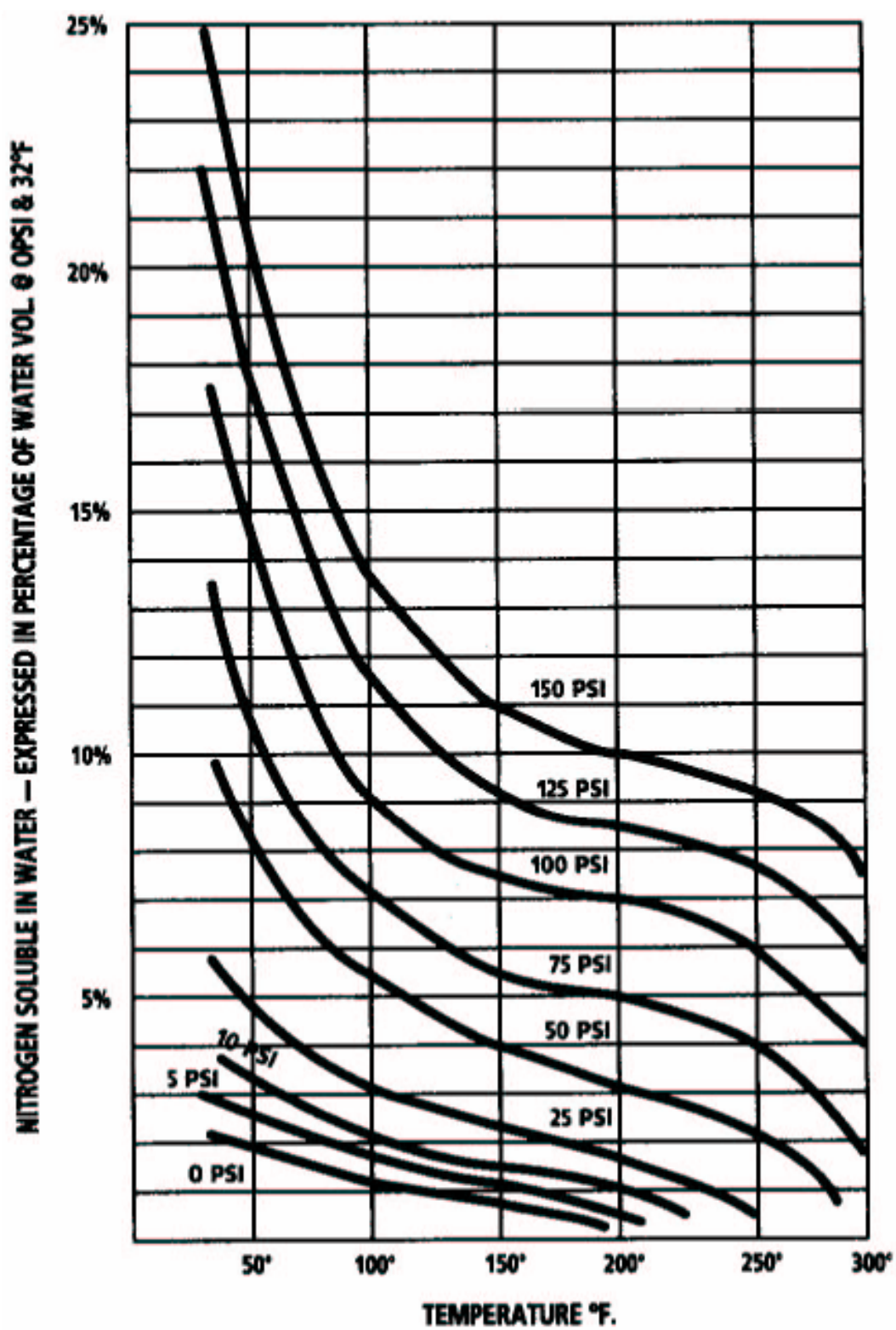

Figure 52 Nitrogen solubility in water as a function of temperature and pressure (Bell and Gossett, 1998). 
It is possible to quantify the purge penalty associated with a direct-contact condenser in which water from a cooling tower is saturated with air and throttled into the system. The amount of air that must be purged from the system in this situation is approximately $1200 \mathrm{cfm}$ (volumetric flow rate at the condenser pressure). In the bestcase scenario, assuming that the air can be completely separated from the refrigerant water vapor and purged from the system, the purging unit would have to pump $1200 \mathrm{cfm}$ of air from the condenser. For a more realistic scenario, it is assumed that half refrigerant water vapor and half air is pulled from the system, resulting in a $2400 \mathrm{cfm}$ load on the purger. Figure 53 shows how the electrical power requirements for several commercially available vacuum pumps vary with flow capacity (Cole-Parmer, 2003). The best fit line to the data is also shown in Figure 53.

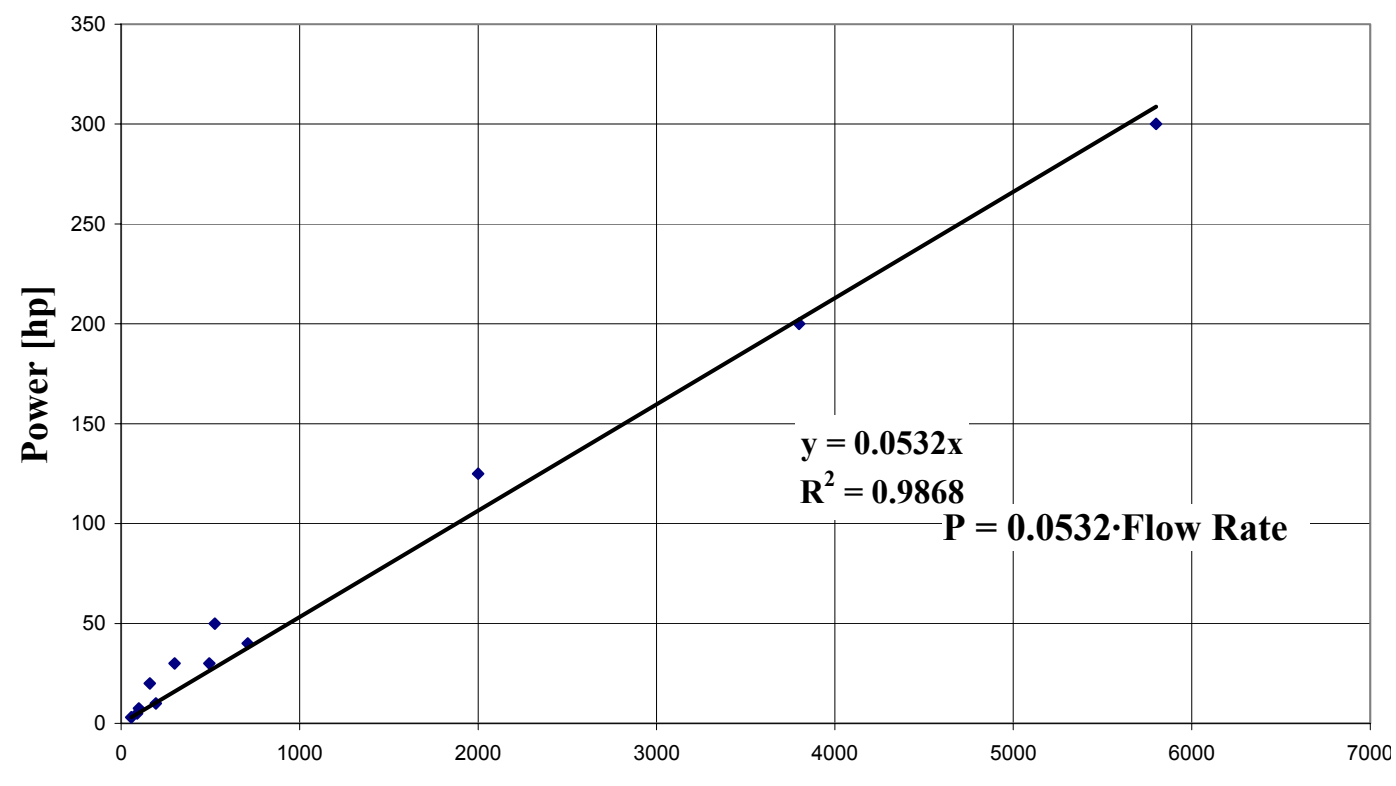

Flow Rate [cfm]

Figure 53 Approximate pump power requirement versus air flow rate.

Based on the approximate pump performance indicated by Figure 53, it is possible to estimate that the input power for the best case scenario, $1200 \mathrm{cfm}$, is $64 \mathrm{hp}$ and for a more realistic scenario, $2400 \mathrm{cfm}$, is $128 \mathrm{hp}$. This is between 10 and 20 percent 
of the total input power to the cycle and therefore represents a comparable and very significant penalty on the overall cycle COP. 


\section{CHAPTER 5 SYSTEM ANALYSIS}

The component level models communicate with the main program in order to form a complete vapor compression refrigeration cycle. These component level models receive inputs that can be used as variables to minimize the life-cycle cost of the refrigeration system. The following sections describe the optimization of the R-134a cycle as well as the optimization of the four water cycle permutations presented in Chapter 4.

\subsection{Economic Model}

Economics will determine whether a water as refrigerant cycle will be feasible when compared with more typical refrigerants like R-134a and others. First costs (capital costs) can vary widely between two chillers, and yet they may have similar life-cycle costs provided that a reduction in operating costs balances a higher capital cost. Because a water-based vapor compression refrigeration cycle has very large and expensive compressors relative to those of other typical refrigerants, it must also possess a higher COP in order for it to be economically attractive on a life-cycle basis. A greater the difference between COPs results in a faster payback time for water as a refrigerant. This section describes the economic model used to evaluate the life-cycle cost for the systems analyzed.

The life-cycle cost (LCC) of a refrigeration unit can be evaluated using a present worth analysis, which takes into consideration capital, operating, and other financial considerations. Fiscal parameters that pertain to a refrigeration system investment decision include tax rates: income tax, property tax, and others. Also, such monetary 
figures as interest rates and inflation rates figure heavily into such a large investment. Other important factors include the expected length of service, maintenance costs, and applicable loan duration. This large number of factors included make an economic analysis somewhat of a test of foresight. The $P_{1}, P_{2}$ approach described here simplifies this analysis by consolidating these variables into two parameters. This approach is attractive in that it becomes relatively easy to determine how sensitive any conclusion is to either of these two parameters and, by extension, how sensitive the result is to changes in the economic factors that contribute to these parameters.

$P_{1}, P_{2}$ Approach

Understanding the time value of money is imperative to making wise investments. The life-cycle cost associated with investing in either an R-134a cycle or a water as a refrigerant cycle is evaluated using the $\mathrm{P}_{1}, \mathrm{P}_{2}$ method. $\mathrm{P}_{1}$ is a multiplier that takes the present value of future energy costs into consideration. It modifies the operating cost so that it will be comparable in real dollars to the capital cost that is incurred up-front. $\mathrm{P}_{1}$ is defined by the following equation.

$$
P_{1}=(1-t) \cdot P W F(N, f, d)
$$

where

$t$ is the effective tax rate $(\%)$

$\mathrm{N}$ is the period of economic analysis (in years)

$\mathrm{f}$ is the fuel inflation rate $(\%)$

$\mathrm{d}$ is the market discount rate $(\%)$

$\mathrm{P}_{2}$ is a multiplier that takes all of the economic parameters pertaining to capital costs into consideration. This multiplier varies depending on amount of the capital cost 
financed, applicable taxes, projected inflation rates, and other considerations. $\mathrm{P}_{2}$ is defined by the following equation.

$$
\begin{aligned}
& P_{2}=D+(1-D) \cdot \frac{\operatorname{PWF}\left(N_{1}, 0, d\right)}{\operatorname{PWF}\left(N_{L}, 0, m\right)} \\
& -t \cdot(1-D) \cdot\left[\operatorname{PWF}\left(N_{1}, m, d\right) \cdot\left(m-\frac{1}{\operatorname{PWF}\left(N_{L}, 0, m\right)}\right)+\frac{\operatorname{PWF}\left(N_{1}, 0, d\right)}{\operatorname{PWF}\left(N_{L}, 0, m\right)}\right] \\
& +[p \cdot(1-t)+M \cdot(1-c \cdot t)] \cdot \operatorname{PWF}(N, i, d)-\frac{c \cdot t}{N_{D}} \cdot \operatorname{PWF}\left(N_{2}, 0, d\right)-S-r
\end{aligned}
$$

where

$\mathrm{D}$ is the ratio of down payment to initial investment

$\mathrm{m}$ is the annual mortgage interest rate (\%)

$i$ is the general inflation rate $(\%)$

$\mathrm{N}$ is the period of economic analysis (in years)

$\mathrm{N}_{\mathrm{L}}$ is the term of loan (in years)

$\mathrm{N}_{1}$ is the number years over which mortgage payments contribute to the analysis (usually the minimum of $\mathrm{N}$ or $\mathrm{N}_{\mathrm{L}}$ )

$\mathrm{N}_{\mathrm{D}}$ is the depreciation lifetime in years

$\mathrm{N}_{2}$ is the number of years over which depreciation contributes to the analysis usually the minimum of $\mathrm{N}$ or $\mathrm{N}_{\mathrm{D}}$ )

$\mathrm{M}$ is the ratio of maintenance, insurance, and other incidental costs to the first costs

$\mathrm{p}$ is the property tax rate based on assessed value $\mathrm{c}$ is the either 1 for commercial investment or 0 for a residential investment 
$t$ is the effective income tax rate where

Effective Tax Rate $=$ Fed Tax Rate + State Tax Rate - Fed Tax Rate $\cdot$ State Tax Rate

$\mathrm{S}$ is the ratio of resale value at end of period of analysis to initial investment

$r$ is the effective state and federal income tax rate

$\mathrm{P}_{2}$ is better understood when broken down into its terms. The first term, $\mathrm{D}$, is simply a ratio of the down-payment to the initial principal investment. The downpayment ratio is not multiplied by a present worth factor, because it is paid in present dollars. The second term is present worth of the loan taken to pay the remainder of the principal investment, (1-D). The third term, $t(1-D)$, is related to the present value of the income tax benefit of interest payments over the term of the loan. The fourth term, $\mathrm{p}(1-$ t), is related to the present worth of the cost of property tax for owning the piece of refrigeration equipment. The fifth term, $M(1-c t)$, is the present value of the refrigeration system maintenance cost. This term also accounts for the fact that maintenance cost is tax deductible for business. The sixth term, $\mathrm{ct} / \mathrm{N}_{\mathrm{D}}$, accounts for the present worth of depreciation for tax purposes. Note that this does not take into consideration the Modified Accelerated Cost Recovery System (MACRS) depreciation schedule, but rather is an approximation for linear depreciation. The final terms, the ratio of the resale value to the initial principal investment $\mathrm{S}$ and the effective state and federal tax rates, $\mathrm{r}$, are not multiplied by present worth factors. $\mathrm{S}$ is the salvage value at the end of the analysis period in present dollars and $r$ is assumed to be a constant rate over the economic analysis period. 
Using these multipliers, the life-cycle cost (LCC) is defined by the following equation in real (today's) dollars.

where

$$
\mathrm{LCC}=\mathrm{P}_{1} \cdot \mathrm{C}_{\mathrm{O}}+\mathrm{P}_{2} \cdot \mathrm{C}_{\mathrm{F}}
$$

$\mathrm{C}_{\mathrm{O}}$ is the first year operating cost

$\mathrm{C}_{\mathrm{F}}$ is the capital cost (first cost)

Determining the first cost of each component has been described section-bysection in Chapter 4. For comparison of water with R-134a, an equal number of "equivalent full-load hours"4 was selected, based on a chiller operating in an office building in Miami, FL (requiring a relatively large number of "equivalent full load hours"). It has been established that the R-134a cycle has a lower COP than the optimal flash intercooled water as refrigerant cycle. Therefore, basing the required "equivalent full-load hours" in Miami will cause the water cycle to appear better economically relative to R-134a than if comparing based on the "equivalent full-load hours" in Madison, WI. The first year operating cost used for the following optimization is based on approximate current energy costs (assumed to be $\$ 0.08 / \mathrm{kW}-\mathrm{h}$, Wisconsin Public Service Corporation, 2004) and "equivalent full-load hours" for a chiller operating in Miami, FL (The Trane Co., 1965).

\subsection{System Optimization}

Using the $\mathrm{P}_{1}, \mathrm{P}_{2}$ approach for economic analysis, the sizes of the components within the system can be varied to minimize life-cycle cost subject to a constraint (refrigeration system capacity). The key components include the heat exchangers and 
compressors. The sizes of the compressors are fixed by the required refrigeration load and system requirements. Here, the sizes of the heat exchangers are optimized by varying their approach temperatures to minimize the life-cycle cost. Pressure drop is set as a design parameter and is assumed to be equal for both indirect and direct-contact heat exchangers. Both parameters are strong levers for optimizing the operating costs and the initial capital cost. Cost data is well established in R-134a cycles and these figures are used as a basis to extrapolate life-cycle costs for the four water cycles. The R-134a system is calibrated to match closely with state-of-the-art refrigeration systems.

\subsubsection{Conventional R-134a System}

A conventional R-134a system is a single-stage, non-intercooled cycle with only indirect-contact heat exchangers. As a review of the analysis completed in Chapter 4, the component costs predicted by the R-134a cycle model are shown in Table 31 . The predictions are compared with the costs associated with state-of-the-art components in an identically sized cycle, provided by several major chiller manufacturers (ARTI, 2004).

Table 31 Individual component costs.

\begin{tabular}{l|c|c} 
& $\begin{array}{c}\text { Cost } \begin{array}{c}\text { Reported } \\
\text { by Chiller } \\
\text { Manufacturers* }\end{array} \\
\text { Component }\end{array}$ & Predicted Cost \\
\hline Compressor & $\$ 20,000$ & $\$ 22,300$ \\
Condenser & $\$ 18,300$ & $\$ 18,000$ \\
Evaporator & $\$ 19,400$ & $\$ 19,900$
\end{tabular}

*average of several responses from various manufacturers

The ability of the model to predict both the size and price of the compressor over a range of refrigeration capacity is shown in Table 32. Figures 54 and 55 illustrate the predicted size and cost of a compressor for an R-134a cycle as a function of capacity.

\footnotetext{
${ }^{4}$ Equivalent full-load hours is an estimate of the number of hours a chiller system would have to run at fullload to equal to actual full- and part-load hour requirements throughout a normal year.
} 
Note that the good agreement between the model and the industry response ensures a relatively accurate prediction of the initial cost associated with the conventional refrigerant cycle. Also, the ability of the model to accurately predict impeller size and compressor cost over a range of capacity lends some confidence in its ability to extrapolate to the significantly larger sizes associated with the water as a refrigerant cycles.

Table 32 Comparison of average compressor size and cost with model predictions for

\begin{tabular}{c|c|c|c|c}
$\begin{array}{c}\text { Capacity } \\
\text { (tons) }\end{array}$ & $\begin{array}{c}\text { Tip diameter } \\
\text { (inch) reported by } \\
\text { Industry* }\end{array}$ & $\begin{array}{c}\text { R-134a chiller. } \\
\text { Tip diameter } \\
\text { (inch) predicted by } \\
\text { model }\end{array}$ & $\begin{array}{c}\text { Cost (\$) } \\
\text { reported by } \\
\text { industry* }\end{array}$ & $\begin{array}{c}\text { Cost (\$) } \\
\text { predicted by } \\
\text { model }\end{array}$ \\
\hline 150 & 5.01 & 4.8 & 10,470 & 7,100 \\
250 & $N / A$ & 6.2 & 12,430 & 9,600 \\
350 & 8.34 & 7.33 & 13,980 & 11,700 \\
500 & 9.17 & 8.76 & 13,990 & 14,500 \\
1000 & 12.54 & 12.32 & 20,050 & 22,300 \\
1500 & 15.4 & 14.95 & 31,750 & 28,700
\end{tabular}

*average of several responses from various manufacturers

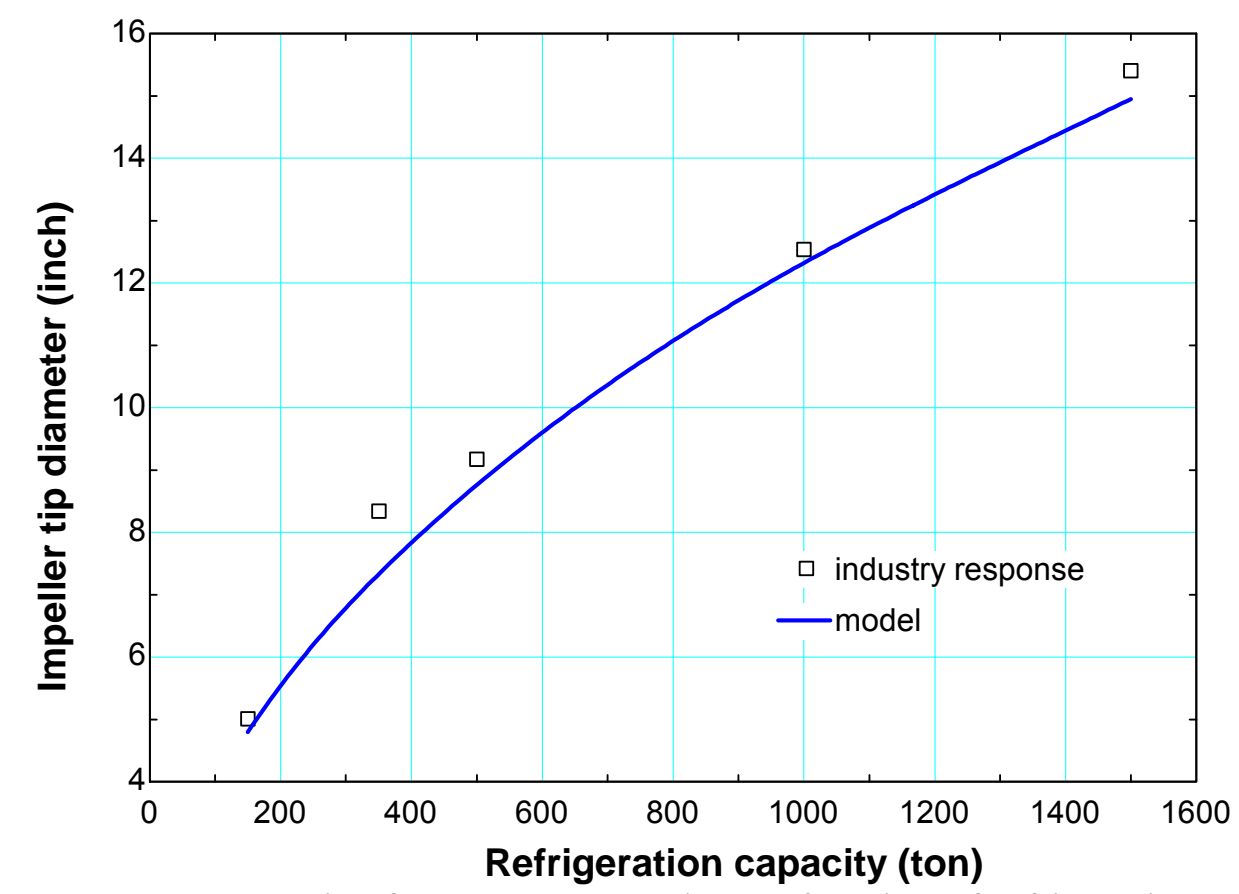

Figure 54 Compressor size for an R-134a cycle as a function of refrigeration capacity reported by industry and predicted by model. 


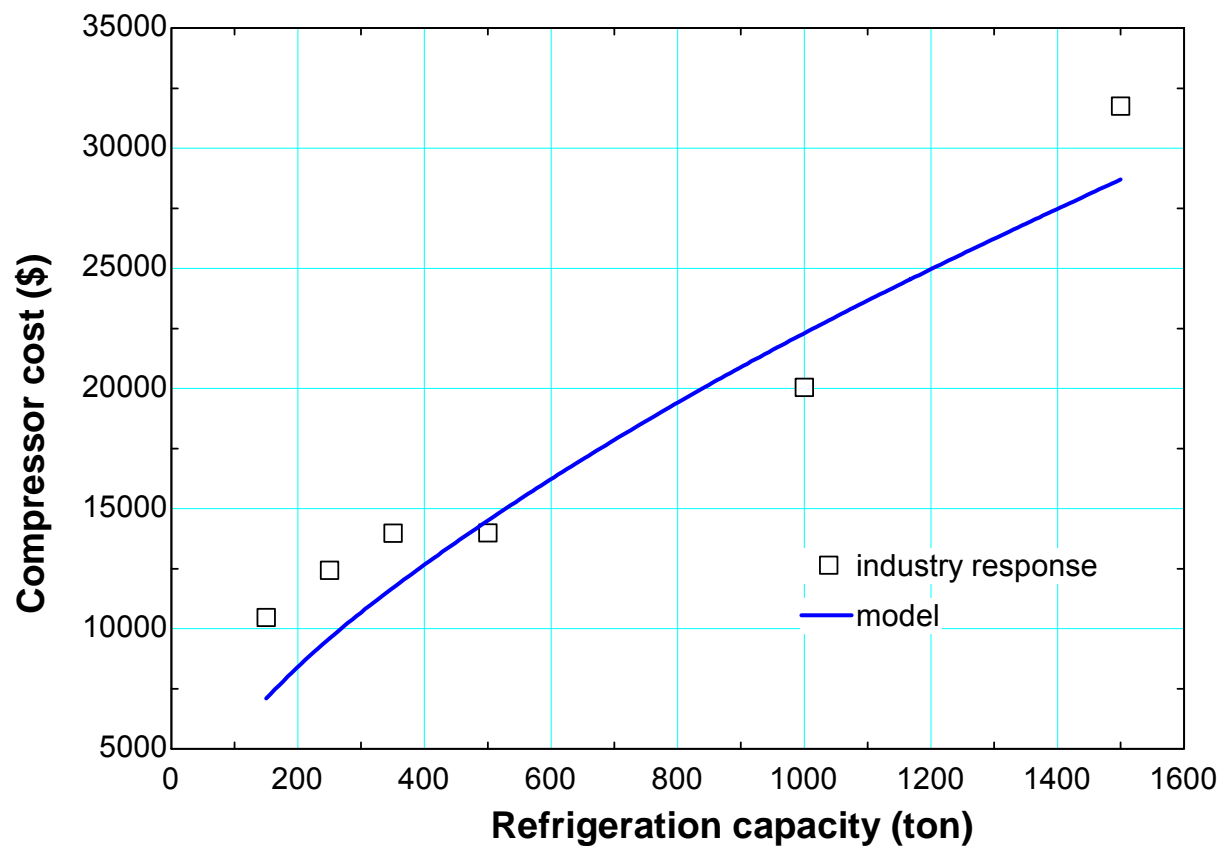

Figure 55 Compressor cost for an R-134a cycle as a function of refrigeration capacity reported by industry and predicted by model.

Table 33 illustrates the ability of the model to accurately predict the size, characteristics, and cost of an evaporator for a 1000 ton chiller.

Table 33 Comparison of industry average R-134a evaporator with model predictions.

Characteristic

Number of tubes

Shell diameter, in

Shell length, $f t$

Approach temperature, ${ }^{\circ} \mathrm{F}$

Refrigerant pressure, psia

Cost

* average of several responses from various manufacturers Predicted by model

\begin{tabular}{c|c}
644 & 690 \\
42.8 & 42 \\
13.3 & 14 \\
1.23 & 1.23 \\
52.6 & 52.5 \\
$\$ 19,400$ & $\$ 19,900$
\end{tabular}

$\$ 19,900$

Table 34 illustrates the ability of the model to accurately predict the size, characteristics, and cost of a condenser for a 1000-ton chiller. 
Table 34 Comparison of industry average R-134a condenser with model predictions.

\begin{tabular}{|c|c|c|}
\hline Characteristic & Reported by industry & Predicted by model \\
\hline Number of tubes & 930 & 910 \\
\hline Shell diameter, in & 36.3 & 36 \\
\hline Shell length, $f t$ & 13.3 & 13 \\
\hline Approach temperature, ${ }^{\circ} \mathrm{F}$ & 1.42 & 1.41 \\
\hline Refrigerant pressure, psia & 125.4 & 131.5 \\
\hline Cost & $\$ 18,300$ & $\$ 18,000$ \\
\hline
\end{tabular}

\subsubsection{Water, Indirect-Contact Condenser/Indirect-Contact Evaporator}

\section{System}

For a water as refrigerant cycle with an indirect-contact condenser and evaporator, the life-cycle cost is expected to be higher than for any of the other permutations due to the relatively high capital cost of indirect heat exchangers as compared with the directcontact heat exchangers.

The optimal size of the direct-contact heat exchangers are obtained by varying the pinch-point temperature difference of the evaporator and condenser. Figures 56 through 58 illustrate the operating cost, capital cost, lifecycle cost and as a function of the evaporator pinch-point temperature difference (all else fixed). Note that a small pinchpoint temperature difference implies a large capital cost and a low operating cost while a large pinch-point temperature difference leads to a small capital cost but a larger operating cost. In between these extremes lies a pinch-point temperature difference that minimizes the life cycle cost. 


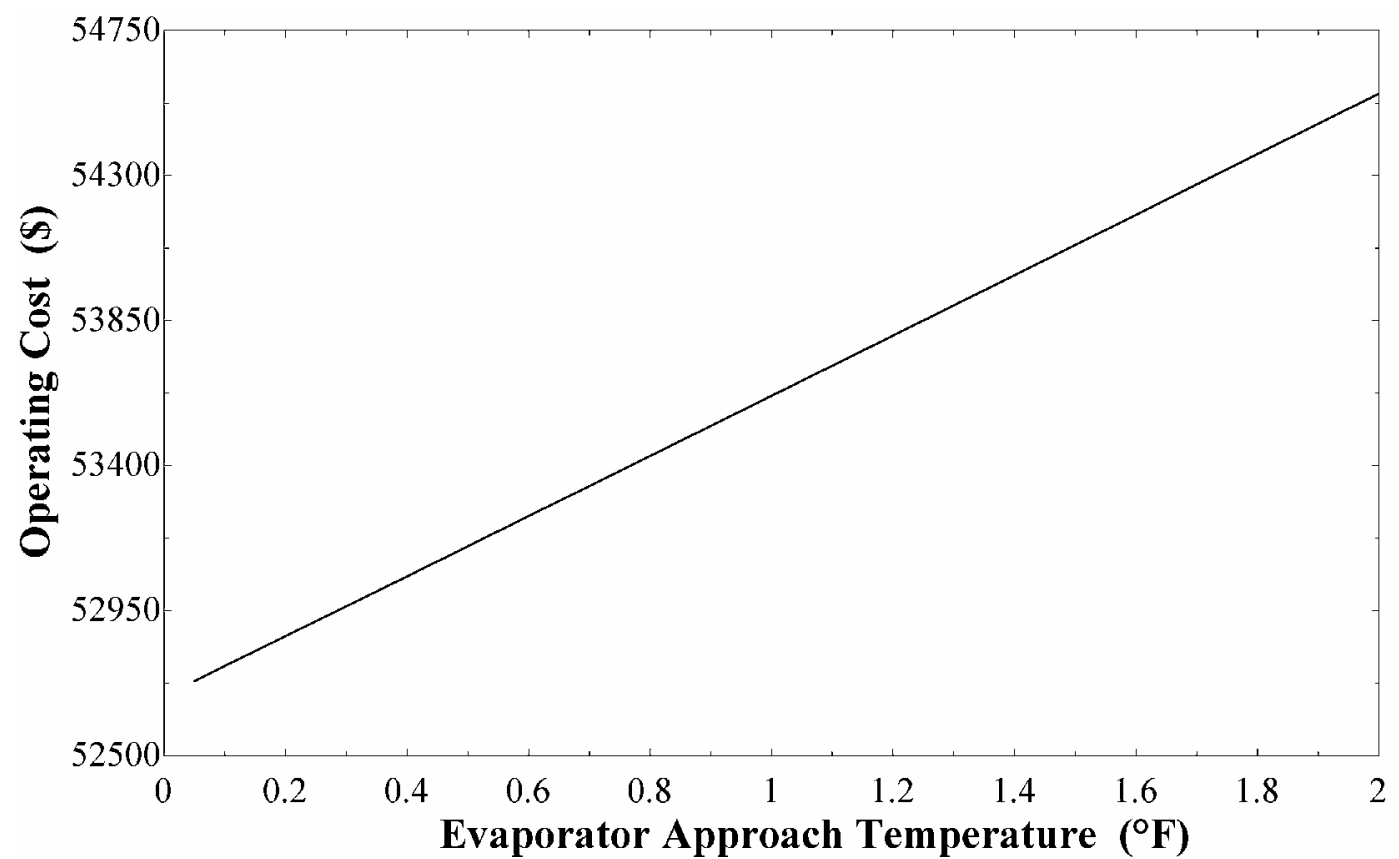

Figure 56 Operating cost as a function of evaporator pinch-point temperature difference for an indirect condenser/indirect evaporator water cycle.

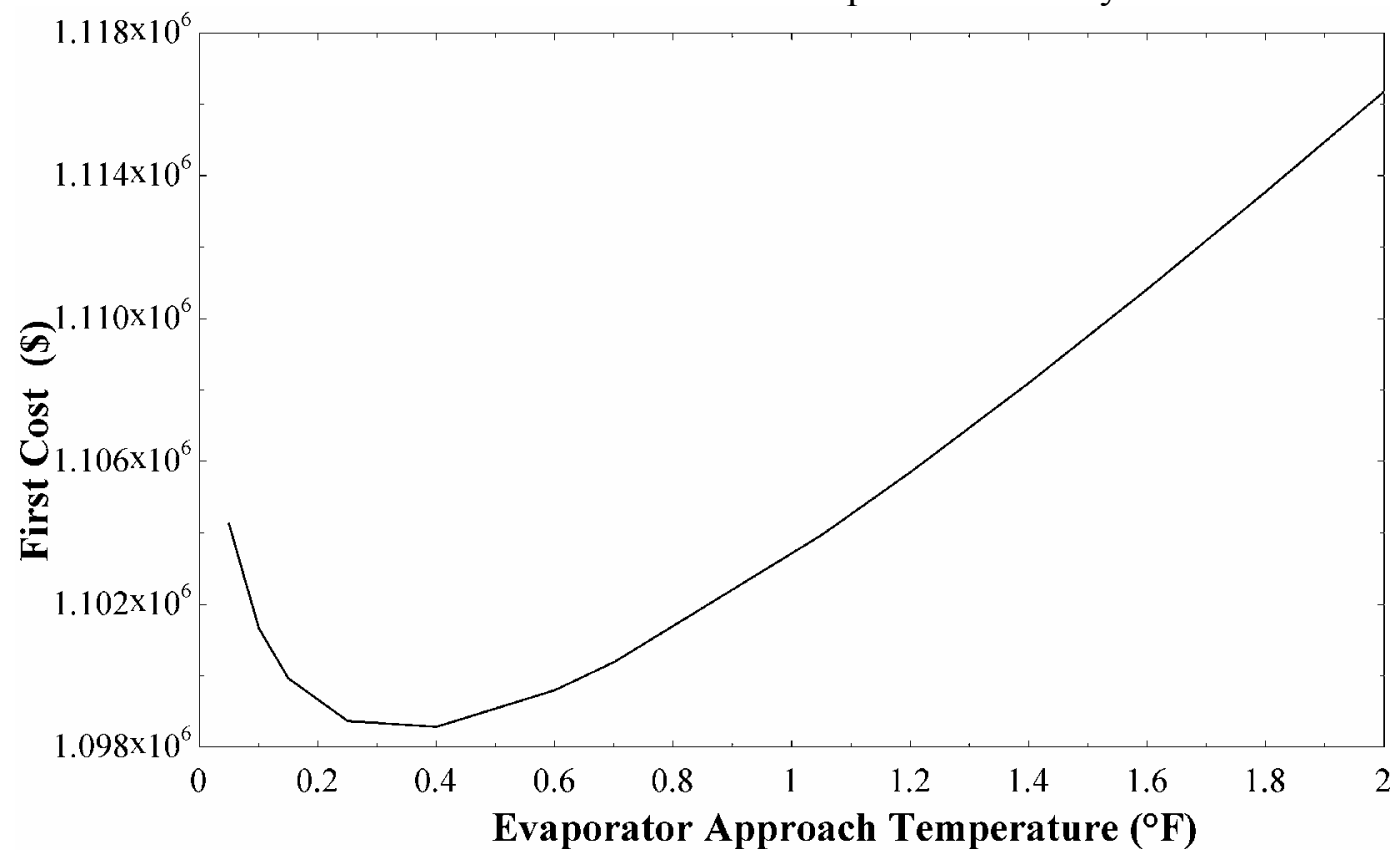

Figure 57 First capital cost as a function of evaporator pinch-point temperature difference for an indirect condenser/indirect evaporator water cycle. 


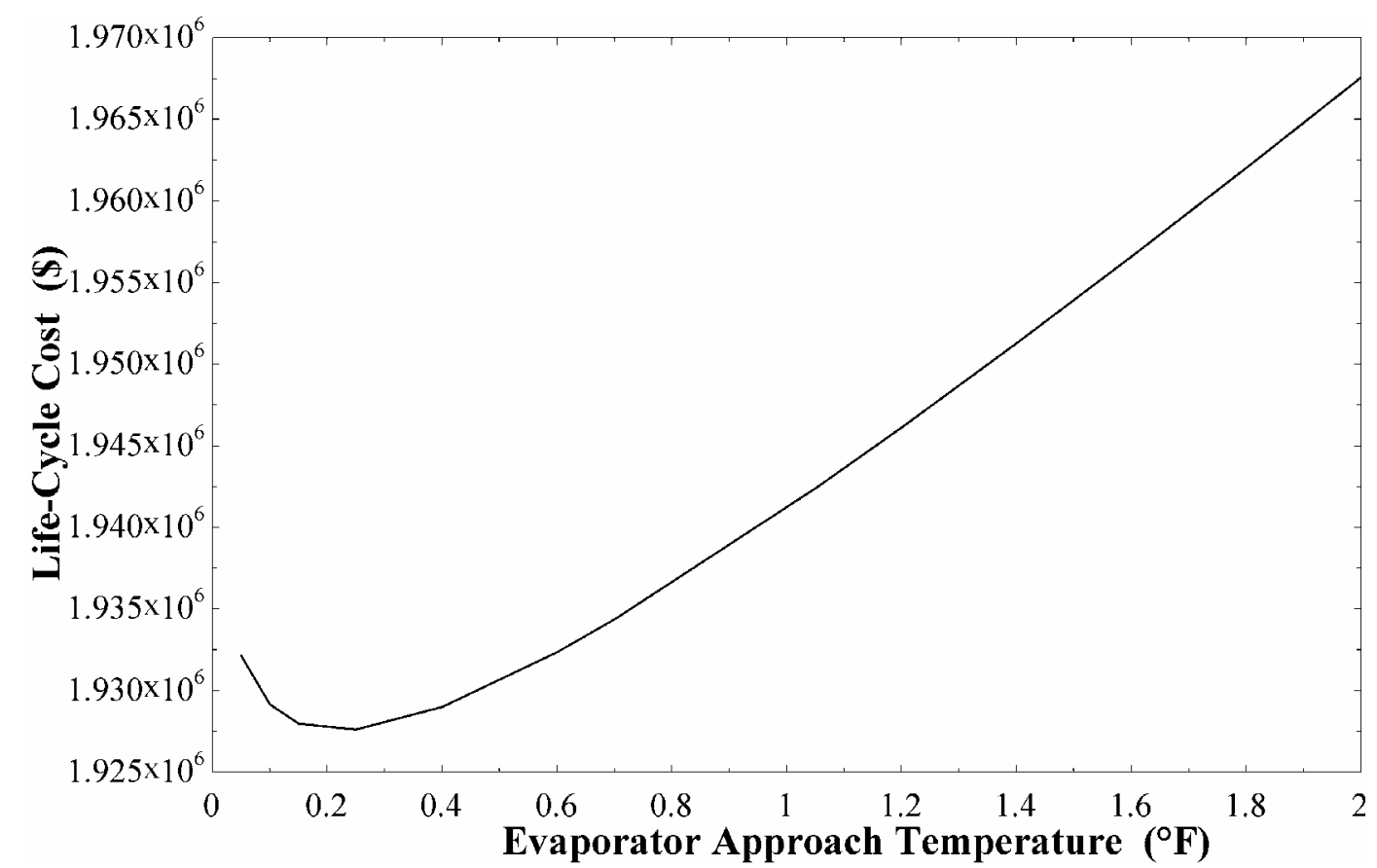

Figure 58 Life-cycle cost as a function of evaporator pinch-point temperature difference for an indirect condenser/indirect evaporator water cycle.

Figure 59 illustrates the life-cycle cost as a function of the condenser pinch-point illustrating that a similar optimal sized condenser exists that minimizes the life-cycle costs. 


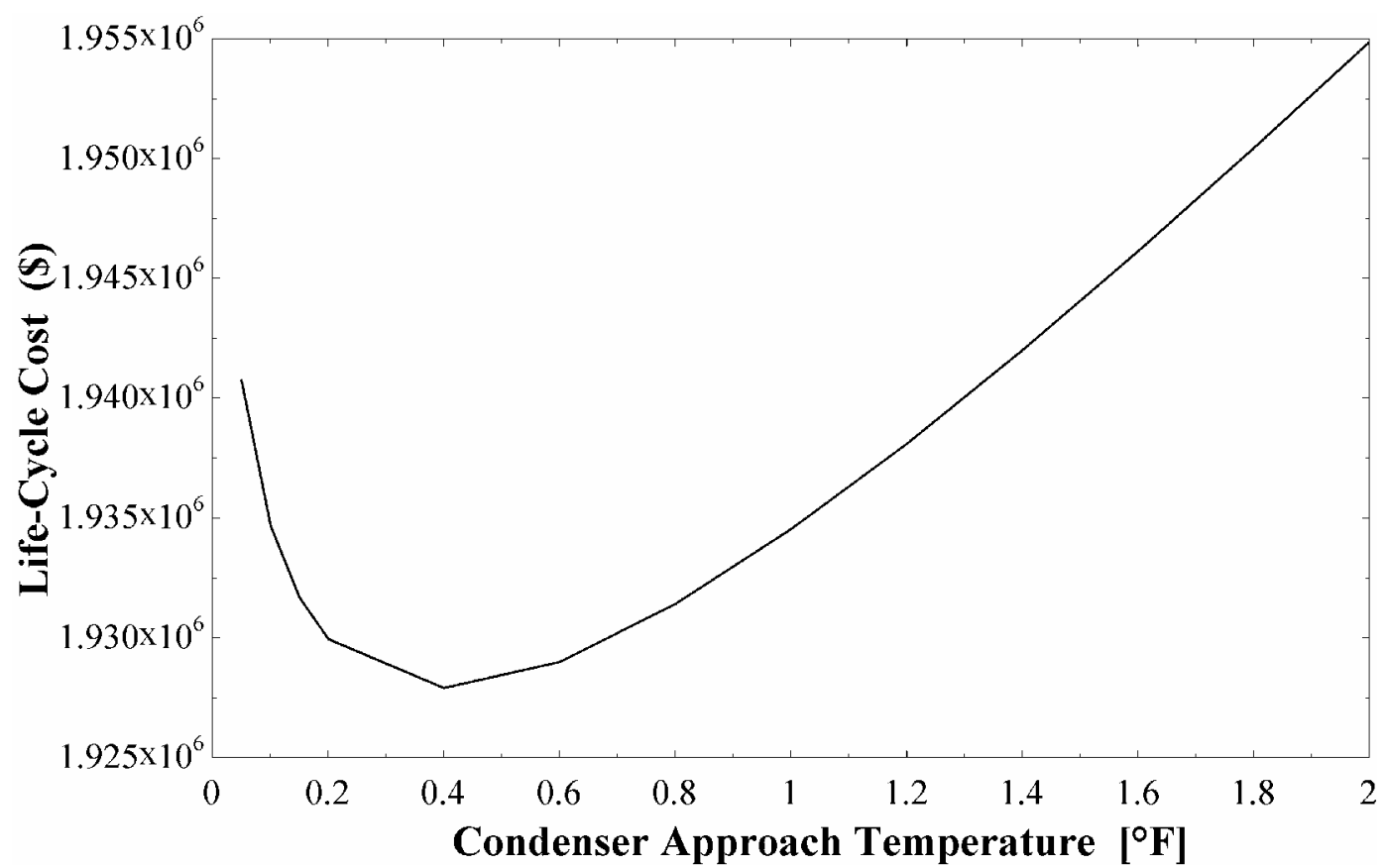

Figure 59 Life-cycle cost as a function of condenser pinch-point temperature difference for an indirect condenser/indirect evaporator water cycle.

Although these components were optimized separately it can be shown that the optimal sizes are relatively unaffected over a broad range. For this optimized cycle, the costs of components associated with a 1000-ton water as refrigerant cycle are compared with the R-134a cycle in Table 35. Tables 36 and 37 compare the optimal evaporator and condenser configurations for these cycles. One of the most striking differences between the R-134a and water cycles lies in the cost of the compressors. As discussed in Section 1.1.1, there are two compressors required for the water cycle, both of which are significantly larger and more expensive than required for the R-134a cycle.

Table 35 Cost comparison of components in 1000-ton water (indirect condenser/indirect evaporator) and R-134a chiller cycles.

\begin{tabular}{l|l|l} 
Component & $\begin{array}{l}\text { R-134a Model } \\
\text { Predicted Cost }\end{array}$ & $\begin{array}{l}\text { Water Model } \\
\text { Predicted Cost }\end{array}$ \\
\hline Compressor \#1 & $\$ 20,900$ & $\$ 668,400$ \\
Compressor \#2 & N/A & $\$ 329,700$ \\
Condenser & $\$ 18,300$ & $\$ 49,500$ \\
Evaporator & $\$ 19,900$ & $\$ 37,600$
\end{tabular}


Table 36 Comparison of water (indirect condenser/indirect evaporator) and R-134a evaporators for 1000-ton chiller systems.

\begin{tabular}{l|c|c} 
Evaporator & $\mathbf{R}-134 \boldsymbol{a}$ Model & Water Model \\
\hline Number of tubes & 690 & 715 \\
Shell diameter, in & 42 & 62 \\
Shell length, $\mathrm{ft}$ & 14 & 20.5 \\
Approach temperature, ${ }^{\circ} \mathrm{F}$ & 1.23 & 0.3 \\
Refrigerant pressure, psia & 52.5 & 0.14 \\
Cost & $\$ 19,900$ & $\$ 37,600$
\end{tabular}

Table 37 Comparison of water (indirect condenser/indirect evaporator) and R-134a condensers for 1000-ton chiller systems.

\begin{tabular}{l|c|c} 
Condenser & $\boldsymbol{R}-134 \boldsymbol{a}$ Model & Water Model \\
\hline Number of tubes & 910 & 900 \\
Shell diameter, in & 36 & 66 \\
Shell length, $\mathrm{ft}$ & 13 & 24 \\
Approach temperature, ${ }^{\circ} \mathrm{F}$ & 1.41 & 0.4 \\
Refrigerant pressure, psia & 131.5 & 0.83 \\
Cost & $\$ 18,300$ & $\$ 49,500$
\end{tabular}

\subsubsection{Water, Direct-Contact Condenser/Indirect-Contact Evaporator System}

For a water-based vapor compression chiller system using a direct-contact condenser and an indirect-contact evaporator, the overall cost of the system should be improved compared to the chiller with both indirect-contact heat exchangers. In fact, the cost of the direct-contact condenser for water as a refrigerant is significantly less than the corresponding R-134a condenser. Figure 60 illustrates the life-cycle cost of the water as a refrigerant cycle as a function of the pinch-point temperature difference for the directcontact condenser (all else constant). Notice that the optimal pinch-point temperature difference is extremely small for the direct-contact heat exchanger due to the relatively low cost of this device as compared with an indirect-contact heat exchanger. 


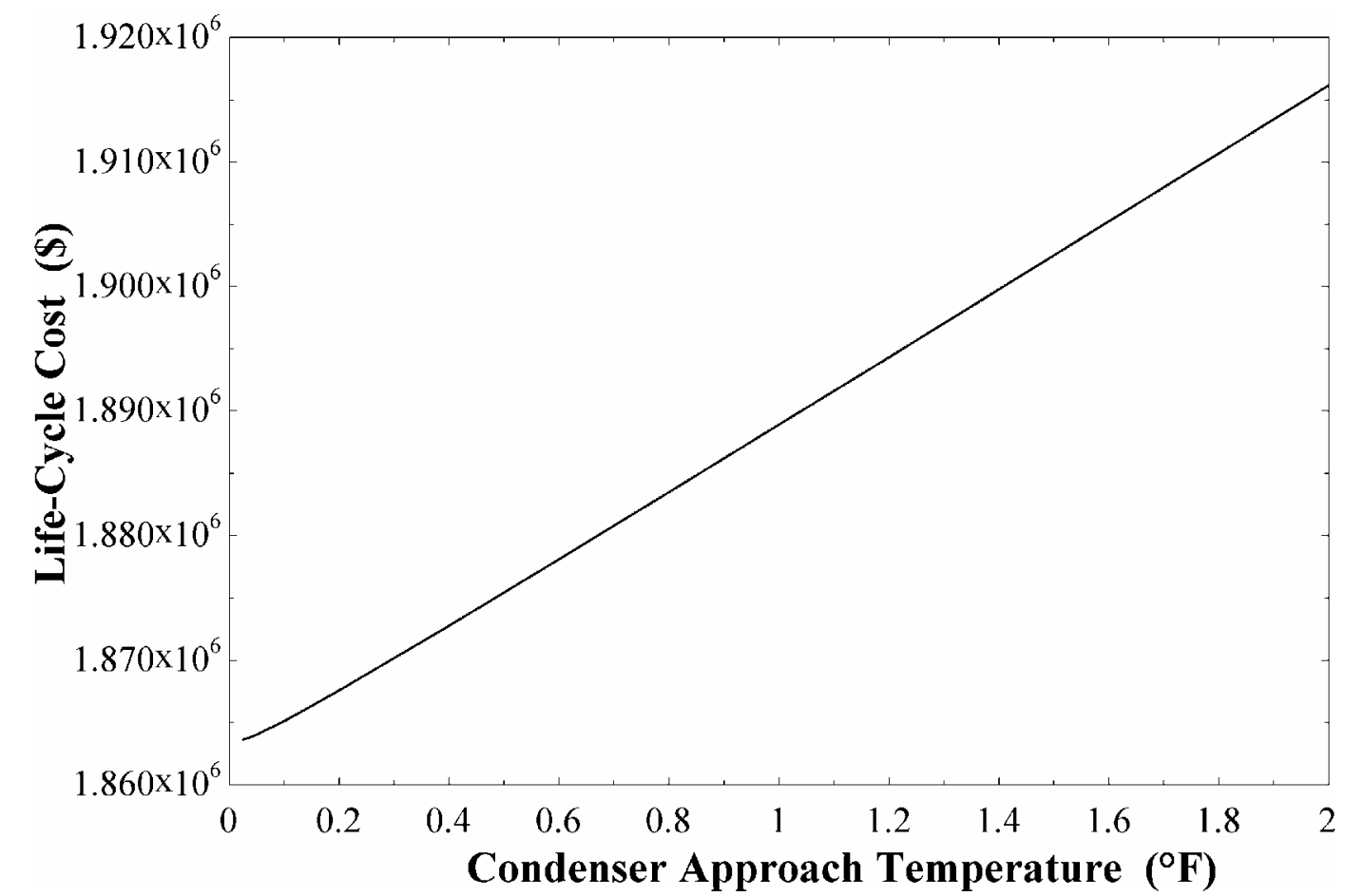

Figure 60 Life-cycle cost as a function of condenser pinch-point temperature difference for a direct condenser/indirect evaporator water cycle.

The results of the optimized component models are compared with the standard R-134a cycle in Tables 38 through 40.

Table 38 Cost comparison of components in 1000-ton water (direct condenser/indirect evaporator) and R-134a chiller cycles.

\begin{tabular}{l|c|c} 
Component & $\begin{array}{c}\boldsymbol{R}-134 a \text { Model } \\
\text { Predicted Cost }\end{array}$ & $\begin{array}{c}\text { Water Model } \\
\text { Predicted Cost }\end{array}$ \\
\hline Compressor \#1 & $\$ 20,900$ & $\$ 665,700$ \\
Compressor \#2 & N/A & $\$ 328,300$ \\
Condenser & $\$ 18,300$ & $\$ 2,400$ \\
Evaporator & $\$ 19,400$ & $\$ 37,600$
\end{tabular}

Table 39 Comparison of water and R-134a indirect-contact evaporators for 1000-ton chiller systems.

\begin{tabular}{l|c|c} 
Evaporator & R-134a Model & Water Model \\
\hline Number of tubes & 690 & 690 \\
Shell diameter, in & 42 & 62 \\
Shell length, $f t$ & 14 & 20.5 \\
Approach temperature, ${ }^{\circ} \mathrm{F}$ & 1.23 & 0.3 \\
Refrigerant pressure, psia & 52.5 & 0.14 \\
Cost & $\$ 19,900$ & $\$ 37,600$
\end{tabular}


Table 40 Comparison of direct-contact water condenser and R-134a indirect-contact condenser for 1000-ton chiller systems.

\begin{tabular}{l|c|c} 
Condenser & R-134a Model & Water Model \\
\hline Number of tubes & 910 & N/A \\
Shell diameter, in & 36 & 97 \\
Shell length, $f t$ & 13 & .95 \\
Approach temperature, ${ }^{\circ} \mathrm{F}$ & 1.41 & 0.025 \\
Refrigerant pressure, psia & 131.5 & 0.83 \\
Cost & $\$ 18,300$ & $\$ 2,400$
\end{tabular}

\subsubsection{Water, Indirect-Contact Condenser/Direct-Contact Evaporator System}

For a water as refrigerant chiller with an indirect-contact condenser and a directcontact evaporator system, the direct-contact evaporator has a beneficial effect on the overall cost of the system. Like the direct-contact condenser, the direct-contact evaporator is not just a net benefit over the indirect-contact evaporator but also over the R-134a evaporator. Figure 61 illustrates the life-cycle cost of a water system with an indirect-contact condenser and a direct-contact evaporator as a function of the directcontact evaporator pinch-point temperature difference. As shown previously with the direct-contact condenser, the optimal pinch-point temperature difference is negligibly small. 


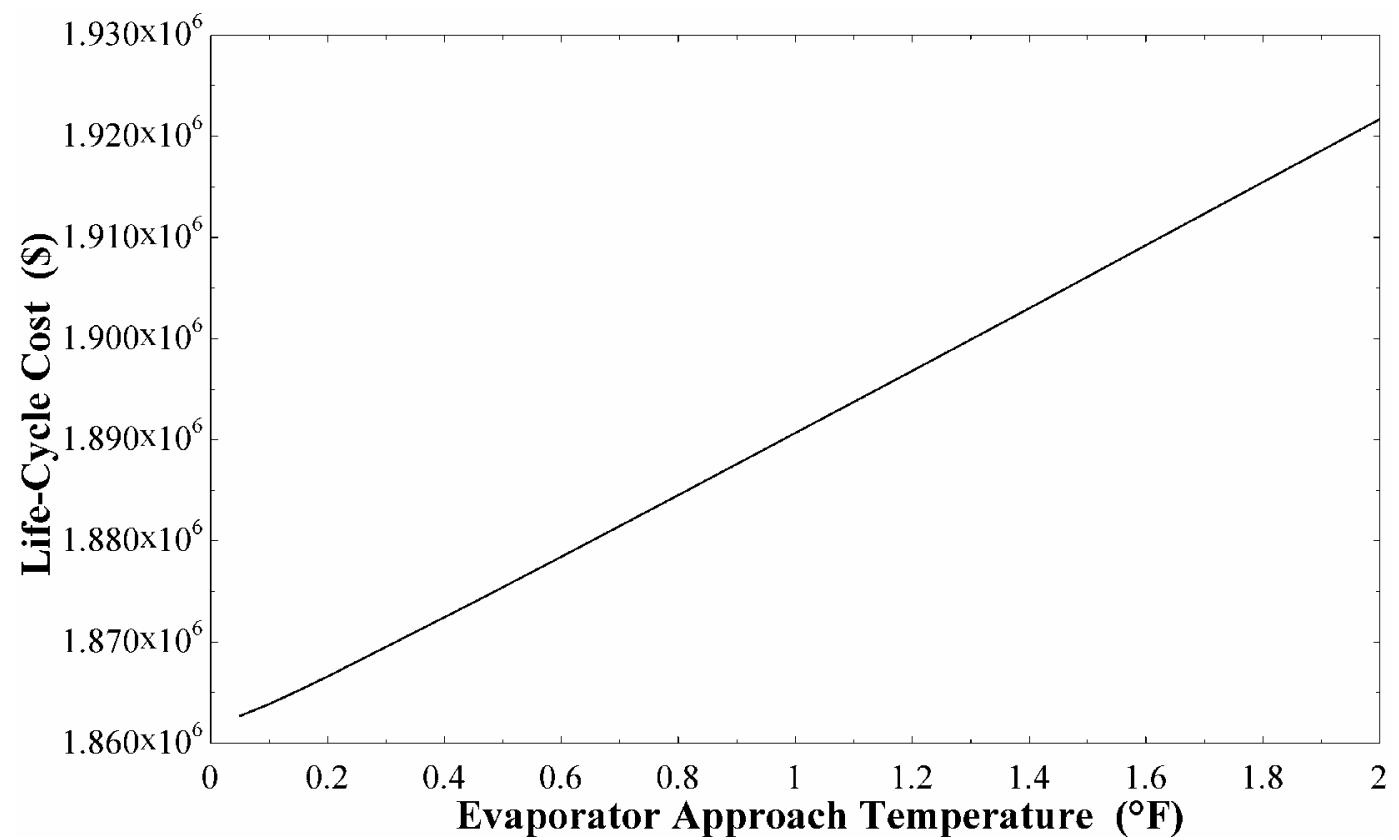

Figure 61 Life-cycle cost of a water cycle with an indirect-contact condenser and directcontact evaporator as a function of the evaporator pinch-point temperature difference.

The results of the optimized indirect-contact condenser and direct-contact evaporator chiller system are compared with those of the R-134a chiller in Tables 41 through 43. It should be noted that the first cost of this cycle and that described in Section 5.2.4 are comparable because they each have one indirect-contact and one directcontact heat exchanger.

Table 41 Cost comparison of components in 1000-ton water and R-134a chiller cycles.

\begin{tabular}{l|l|l} 
Component & $\begin{array}{l}\boldsymbol{R}-134 a \text { Model } \\
\text { Predicted Cost }\end{array}$ & $\begin{array}{c}\text { Water Model } \\
\text { Predicted Cost }\end{array}$ \\
\hline Compressor \#1 & $\$ 20,900$ & $\$ 668,200$ \\
Compressor \#2 & $N / A$ & $\$ 329,500$ \\
Condenser & $\$ 18,300$ & $\$ 30,400$ \\
Evaporator & $\$ 19,400$ & $\$ 3,100$
\end{tabular}


Table 42 Comparison of direct-contact water evaporator and R-134a indirect-contact evaporator for 1000-ton chiller systems.

\begin{tabular}{l|c|c} 
Evaporator & R-134a Model & Water Model \\
\hline Number of tubes & 690 & N/A \\
Shell diameter, in & 42 & 114 \\
Shell length, $\mathrm{ft}$ & 14 & 0.94 \\
Approach temperature, ${ }^{\circ} \mathrm{F}$ & 1.23 & 0.015 \\
Refrigerant pressure, psia & 52.5 & 0.15 \\
Cost & $\$ 19,900$ & $\$ 3,100$
\end{tabular}

Table 43 Comparison of water and R-134a condensers for 1000-ton chiller systems.

\begin{tabular}{l|c|c} 
Condenser & R-134a Model & Water Model \\
\hline Number of tubes & 910 & 900 \\
Shell diameter, in & 36 & 54 \\
Shell length, $\mathrm{ft}$ & 13 & 19 \\
Approach temperature, ${ }^{\circ} \mathrm{F}$ & 1.41 & 0.2 \\
Refrigerant pressure, psia & 131.5 & 0.83 \\
Cost & $\$ 18,300$ & $\$ 30,400$
\end{tabular}

\subsubsection{Water, Direct-Contact Condenser/Direct-Contact Evaporator System}

For a water as refrigerant chiller system, the direct-contact condenser and evaporator permutation is the most attractive from a first cost perspective. A comparison of the components of the standard R-134a cycle with those of a water cycle with directcontact heat exchangers is shown in Tables 44 through 46 . This cycle is selected as the preferred cycle, confirmed by the analysis in the next section.

Table 44 Cost comparison of components in 1000-ton water and R-134a chiller cycles.

\begin{tabular}{l|l|l} 
Component & $\begin{array}{l}\boldsymbol{R}-134 a \text { Model } \\
\text { Predicted Cost }\end{array}$ & $\begin{array}{c}\text { Water Model } \\
\text { Predicted Cost }\end{array}$ \\
\hline Compressor \#1 & $\$ 20,900$ & $\$ 662,400$ \\
Compressor \#2 & N/A & $\$ 326,700$ \\
Condenser & $\$ 18,300$ & $\$ 2,500$ \\
Evaporator & $\$ 19,400$ & $\$ 3,100$
\end{tabular}


Table 45 Comparison of direct-contact water evaporator and R-134a indirect-contact evaporator for 1000-ton chiller systems.

\begin{tabular}{l|c|c} 
Evaporator & R-134a Model & Water Model \\
\hline Number of tubes & 690 & N/A \\
Shell diameter, in & 42 & 115 \\
Shell length, $\mathrm{ft}$ & 14 & 0.95 \\
Approach temperature, ${ }^{\circ} \mathrm{F}$ & 1.23 & 0.015 \\
Refrigerant pressure, psia & 52.5 & 0.15 \\
Cost & $\$ 19,900$ & $\$ 3,100$
\end{tabular}

Table 46 Comparison of direct-contact water condenser and R-134a indirect-contact condenser for 1000-ton chiller systems.

\begin{tabular}{l|c|c} 
Condenser & $\boldsymbol{R}-134 a$ Model & Water Model \\
\hline Number of tubes & 910 & N/A \\
Shell diameter, in & 36 & 100 \\
Shell length, $f t$ & 13 & 0.96 \\
Approach temperature, ${ }^{\circ} \mathrm{F}$ & 1.41 & 0.015 \\
Refrigerant pressure, psia & 131.5 & 0.83 \\
Cost & $\$ 18,300$ & $\$ 2,500$
\end{tabular}

Based on the cost data, the direct-contact condenser and evaporator design is the most attractive permutation of the water cycles. The shell size for both the direct-contact condenser and evaporator is smaller than their indirect-contact counterparts. Also, the packing material is less expensive than the tubing for an equivalent performance. However, this project does not consider the purging issues related to the use of directcontact heat exchangers, in particular a direct-contact condenser. Purging noncondensables from the condenser will result in a significant COP penalty on the cycle.

\subsection{Economic Comparison}

In order for water as a refrigerant to be an economically viable refrigerant, the loss in initial capital cost must at least balance the savings in operating cost over the life of the unit. The break-even initial capital cost can be determined based on the performance projections for the R-134a and water cycles and the capital cost of the R- 
134a cycle. These quantities can be estimated most accurately and therefore the breakeven capital cost of the water cycle provides a convenient method for presenting the economic results. The breakeven cost of the water cycle is compared with the first cost of the water cycle based on the models described here. This first cost estimate requires a significant extrapolation from currently manufactured equipment and therefore has some significant uncertainty associated with it.

The following equation is used to determine the break even first cost.

$$
\mathrm{C}_{\mathrm{F}, \text { water }}=\frac{\mathrm{P}_{1} \cdot \mathrm{C}_{\mathrm{O}, \mathrm{R} 134 \mathrm{a}}+\mathrm{P}_{2} \cdot \mathrm{C}_{\mathrm{F}, \mathrm{R} 134 \mathrm{a}}-\mathrm{P}_{1} \cdot \mathrm{C}_{\mathrm{O} \text {,water }}}{\mathrm{P}_{2}}
$$

where $\mathrm{C}_{\mathrm{F} \text {,water }}$ is the maximum allowable first cost of a competing water cycle, $\mathrm{C}_{\mathrm{F}, \mathrm{R}-134 \mathrm{a}}$ is the first cost for the R-134a cycle, and $\mathrm{C}_{\mathrm{O} \text {,water }}$ and $\mathrm{C}_{\mathrm{O}, \mathrm{R}-134 \mathrm{a}}$ are the yearly operating costs associated with a water and R-134a cycle, respectively. For the four possible permutations of the flash intercooled water cycle considered, Table 47 summarizes the results of a 20 -year life cycle analysis. Table 47 shows both the maximum allowable (based on the R-134a cycle) and the estimated first cost of each water cycle permutation. The permutations correspond to the various combinations of direct and indirect heat exchangers that are possible.

\begin{tabular}{|c|c|c|c|c|}
\hline $\begin{array}{l}\text { Cycle } \\
\text { Configuration }\end{array}$ & $\begin{array}{l}\text { indirect cond. } \\
\text { indirect evap }\end{array}$ & $\begin{array}{l}\text { indirect cond. } \\
\text { direct evap. }\end{array}$ & $\begin{array}{l}\text { direct cond. } \\
\text { indirect evap. }\end{array}$ & $\begin{array}{l}\text { direct cond } \\
\text { direct evap }\end{array}$ \\
\hline Compressor(1) & $\$ 668,400$ & 668,200 & 665,700 & 662,400 \\
\hline Compressor(2) & $\$ 329,600$ & 329,500 & 328,300 & 326,700 \\
\hline Condenser & $\$ 49,500$ & 30,400 & 2,400 & 2,500 \\
\hline Evaporator & $\$ 37,600$ & 3,100 & 37,600 & 3,100 \\
\hline $\begin{array}{l}\text { Predicted Actual } \\
\text { First Cost }\end{array}$ & $\$ 1,098,000$ & $1,045,900$ & $1,048,760$ & $1,007,600$ \\
\hline $\begin{array}{l}\text { Max Allowable } \\
\text { First Cost }\end{array}$ & $\$ 83,700$ & 83,900 & 85,900 & 88,400 \\
\hline Water LCC (20-yr) & $\$ 1,928,000$ & $1,862,000$ & $1,864,000$ & $1,813,000$ \\
\hline$R-134 a$ LCC (20-yr) & $\$ 785,000$ & $N / A$ & N/A & $N / A$ \\
\hline
\end{tabular}


Table 47 indicates that there is no permutation of a water cycle is economically competitive with an R-134a cycle over a 20-year horizon as in each case the predicted actual first cost far exceeds the maximum allowable first cost for the water cycle. Even allowing for the uncertainty associated with the water cycle equipment cost, the large discrepancy between these numbers seems to indicate little chance that the water cycle will be economically competitive. The most attractive water cycle takes advantage of the cheaper, higher performance direct-contact heat exchangers to accomplish both the evaporation and condensation functions; the benefit of this is significant but insufficient to overcome the very high cost of the centrifugal compressors. These compressors cannot together require more than a nominally $\$ 85 \mathrm{k}$ premium when compared to the R134a cycle equipment.

Figure 62 illustrates the maximum allowable first cost as a function of the lifecycle duration for the direct condenser/direct evaporator permutation.

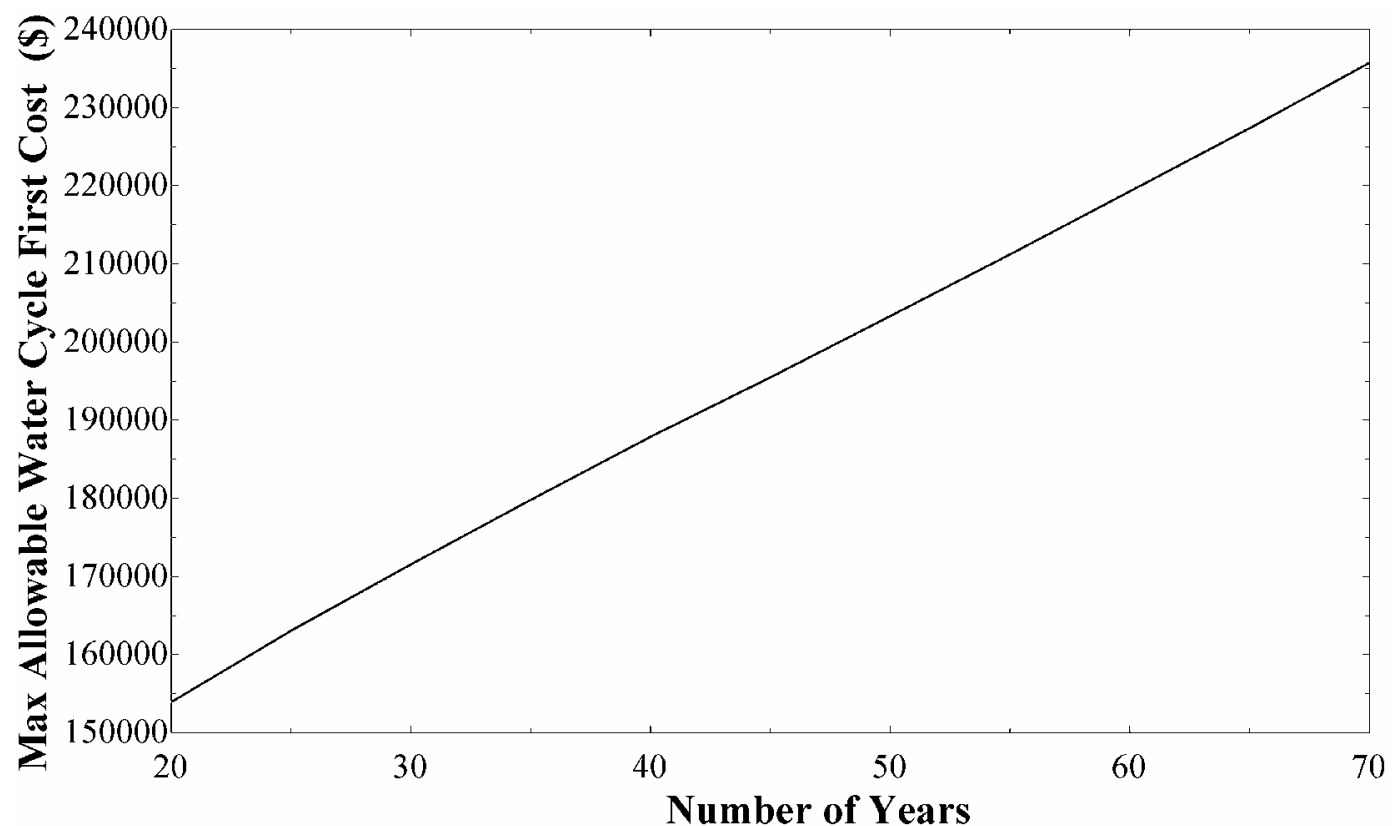

Figure 62 Maximum allowable first cost as a function of the lifetime for a water cycle with a direct-contact condenser and direct-contact evaporator. 
Notice that over an extremely long life-cycle, the maximum allowable first cost does eventually approach the actual first cost. However, the greater likelihood that contamination or fouling issues will degrade the performance of the water cycle over this may eliminate its advantage over the R-134a cycle.

Note that Table 47 provides a convenient means of illustrating the results of this study. The greatest uncertainty in the economic analysis lies in predicting the first cost of a production chiller using water as a refrigerant. However, the maximum allowable first cost that can be tolerated depends only on the current first cost of a centrifugal chiller and the performance of the water and R-134a cycles; these are quantities that can be predicted with some degree of accuracy.

Table 48 indicates the effect that various economic parameters have on the P1 and P2 values. In Table 48, each of the economic inputs is varied by $\pm 20 \%$ and the resulting change in the P1 and P2 parameters are recorded. Table 48 therefore yields some insight into the possible variation in these parameters due to the uncertainties in these economic variables.

Table 48 Effect of economic variables on P1 and P2.

\begin{tabular}{l|c|c|c} 
Variable & Value $\pm \mathbf{2 0} \%$ & $\mathbf{P 1} \pm \boldsymbol{\delta P}_{\mathbf{1}}$ & $\mathbf{P 2} \pm \mathbf{\delta P}_{\mathbf{2}}$ \\
\hline Discount rate & $0.05 \pm 0.01$ & $11.43 \pm 1.143$ & $1.205 \pm 0.146$ \\
Down payment fraction & $0.20 \pm 0.04$ & $11.43 \pm 0$ & $1.205 \pm 0.00178$ \\
Fuel inflation rate & $0.05 \pm 0.01$ & $11.43 \pm 1.034$ & $1.205 \pm 0$ \\
Market interest rate & $0.02 \pm 0.004$ & $11.43 \pm 0$ & $1.205 \pm 0.03242$ \\
Mortgage interest rate & $0.075 \pm 0.015$ & $11.43 \pm 0$ & $1.205 \pm 0.1951$ \\
Depreciation lifetime & $39 \pm 7.8$ & $11.43 \pm 0$ & $1.205 \pm 0.02556$ \\
\hline Term of loan & $20 \pm 4$ & $11.43 \pm 0$ & $1.205 \pm 0.06257$ \\
Period of analysis & $20 \pm 4$ & $11.43 \pm 2.286$ & $1.205 \pm 0.1847$ \\
Property tax & $0.035 \pm 0.007$ & $11.43 \pm 0$ & $1.205 \pm 0.6159$ \\
Salvage fraction & $0.20 \pm 0.04$ & $11.43 \pm 0$ & $1.205 \pm 0.04$ \\
Effective tax rate & $0.40 \pm 0.08$ & $11.43 \pm 1.524$ & $1.205 \pm 0.2773$
\end{tabular}




\subsection{Specification of Preferred Cycle}

Based on the economic analysis presented in Section 5.3, the preferred cycle is the 2-stage flash intercooled cycle with a direct-contact evaporator and condenser. Optimized by approach temperature, Table 49 shows the specifications of the compressors for the preferred cycle. Refer to Tables 45 and 46 for the specifications of the heat exchangers for the direct-contact condenser and evaporator cycle.

Table 49 Preferred cycle compressor specifications.

\begin{tabular}{l|c|c} 
Compressors & L.P. Compressor & H.P. Compressor \\
\hline Tip diameter, $f t$ & 9.2 & 6.1 \\
Rotational speed, rpm & 2,960 & 4,535 \\
Specific speed & 0.519 & 0.522 \\
Cost, \$US & 662,500 & 326,700
\end{tabular}




\section{CHAPTER 6 CONCLUSIONS}

On a thermodynamic and environmental basis, water as a refrigerant compares well with other more typical refrigerants. It has a high theoretical COP, zero ozone depletion potential and a global warming potential less than 1. It is safe and non-toxic and it can be used as both a primary and secondary refrigerant in many applications. However, it has some serious practical drawbacks that are primarily associated with the compression process. The high specific volume vapor at the compressor suction coupled with a large pressure ratio provides a challenging compression process relative to other refrigerants.

The dominant losses in a water-as-refrigerant cycle are related to the superheat during the compression process. The throttling losses for this refrigerant are very small. This observation, coupled with the large pressure ratio required for the cycle, motivated the consideration of various cycle configurations which could provide intercooling. It was found that even one stage of intercooling was capable of elevating the theoretical $\mathrm{COP}$ of a water as refrigerant cycle to slightly above that of a single-stage R-134a cycle. The various methods of intercooling that were explored included economization, indirect intercooling, flash intercooled, liquid subcooling, and direct intercooling.

The economized cycle utilizes the flash gas generated in the expansion process. This gas is a small fraction of the flow due to the high enthalpy of vaporization of water, and this method of intercooling is therefore ineffective. The indirect intercooled cycle is very effective and it raises the COP of the water cycle for one stage of intercooling above that of the R-134a cycle. However, the high capital cost associated with an added indirect-contact heat exchanger and the associated pressure loss makes it less attractive 
than other cycle configurations. Flash intercooling was determined to have the right balance of performance, low pressure drop, and low capital cost. The liquid subcooled cycle and direct intercooled cycles are attractive for higher pressure refrigerants, but the requirement of standing water greatly diminishes the cycle $\mathrm{COP}$ for water as a refrigerant due to the increase in compressor pressure ratio associated with the hydrostatic head.

Based on the preceding discussion, the most promising cycle configuration was determined to be the 2-stage flash intercooled vapor compression cycle. Detailed component level models were developed to allow a more accurate simulation of this system. In order to provide a baseline for comparison, these component-level models were also used to simulate a conventional, R-134a chiller.

The centrifugal compressor model that was developed for this purpose is a meanline, on-design model. The model sufficiently captures the physics associated with the sub-components and processes that together make up the centrifugal compression process in order to allow an accurate specification of size, performance, and cost.

Heat exchanger models were developed to simulate both the shell-and-tube, indirect-contact heat exchangers and the direct-contact heat exchangers. A wetted packing material was found to provide the most attractive direct-contact heat exchange geometry. These models are used to size a heat exchanger for a particular operating condition subject to a specified performance. For water as a refrigerant, the operating pressures are on the order of 0.1 psia. Therefore, pressure drops that would be negligible in other cycles have a profound, negative impact on a water as refrigerant cycle. The need to control the pressure drop in the heat exchangers for the water as refrigerant cycle required that the indirect-contact heat exchangers to be larger and more expensive than 
their R-134a counterparts. However, the heat transfer coefficients for the water cycle were somewhat higher due to the transport properties of water. The higher heat transfer coefficients resulted in a smaller required heat exchange area; however this effect did not overcome the more stringent pressure drop requirement. Thus, heat exchangers in the water cycle were more expensive than for the R-134a cycle. The direct-contact heat exchangers were found to perform better and be much less expensive than any indirectcontact heat exchanger.

In order to verify the performance and geometry predicted by these component models and also calibrate the models used to estimate the cost of these components a survey of three U.S. chiller manufacturers was developed at the UW-Madison and executed by ARTI. The results of the responses were averaged and together used to verify the models and develop cost correlations. It was found that the centrifugal compressor model was able to predict the size and performance of an R-134a compressor for a wide range of refrigeration capacities. The indirect heat exchanger models were capable of predicting the overall size and geometry of the evaporator and condenser within a 1000-ton R-134a chiller.

The 2-stage flash intercooled cycle with direct-contact heat exchangers was determined to be the ideal vapor compression cycle with water as a refrigerant. The lifecycle cost of such a cycle was compared with the life cycle cost of a conventional R134a. The results of this comparison were presented in terms of the predicted actual first cost of the water as a refrigerant cycle and the allowable first cost for the cycle based on the requirement that it be economically equivalent to a conventional chiller over a 20 year life. It was found that the predicted actual first cost was nominally $12 x$ larger than 
what could be economically justified based on the increase in performance. The advantage of presenting the results in this manner is that the major uncertainties in the analysis are concentrated in the actual first cost of a water as refrigerant cycle. The operating costs of the conventional and water as refrigerant cycle and the capital cost of a conventional chiller can be predicted with some accuracy. Therefore, the maximum allowable capital cost for the water cycle can be estimated fairly well. The large discrepancy between the maximum allowable and estimated capital cost for the water cycle indicate that the use of water as a refrigerant is not economically viable now and is unlikely to be economically viable in the near future.

This analysis is somewhat conservative in that it likely overestimates the performance and underestimates the cost associated with the water as a refrigeration cycle. There are several practical issues that were identified but ignored in the analysis. For example, the combination of high pressure ratio and large volumetric flow rate requires the use of a multi-stage centrifugal compressor. The results of the compressor analysis indicate that there is the possibility of condensation and blade erosion at the impeller blade inlet due to the acceleration of the saturated vapor through the suction nozzle.

Also, the use of direct-contact heat exchangers, particularly a direct-contact condenser, is likely to introduce practical issues related to contamination. The secondary refrigerant will be exposed to noncondensables such as air and other contaminants and therefore transport some amount of these undesirable materials into the cycle. Filtration and purging may become a significant problem that would negatively impact the cycle performance. 


\section{REFERENCE LIST FOR WATER VAPOR COMPRESSION CYCLES}

Albring, P., Heinrich, G., 1998, “Turbo Chiller with Water as a Refrigerant”, IIF/IIR Oslo, 1998.

Albring, P., Burandt, B., Heinrich, G., 2000, "Direct Evaporation in Refrigeration Systems with Water as Refrigerant", $20^{\text {th }}$ International Congress of Refrigeration, IIR/IIF, Sydney.

American Society of Heating Refrigerating, and Air-Conditioning Engineers, 1997, "Pocket Guide for Air Conditioning Heating Ventilation Refrigeration", Atlanta, GA.

ARI, Chiller Performance and Cost Information, Personal Communication, Steve Szymurski, (2004).

Barba, D., DiFelice, R., 1984, "Heat Transfer in Turbulent Flow on a Horizontal Tube Falling Film Evaporator. A Theoretical Approach", $8^{\text {th }}$ Int'l Congress of Chemical Engineering, Chemical Equipment Design and Automation, Prague, pp. 325-333.

Balje, O.E., 1981, “Turbomachines: A Guide to Design, Selection, and Theory”, Wiley and Sons, N.Y.

Bell and Gossett/ITT, 1998, "Fluid Handling Training Seminar", Bell and Gossett, Bulletin TEH-1196A.

Braun, J.E., 1988, "Methodologies for the Design and Control of Central Cooling Plants", PhD Thesis, University of Wisconsin - Madison, WI.

Brickman, R.A., Boehm, R.F., 1995, "Numerical Simulation and Comments on Sieve Tray Spray-Column Direct-contact Heat Exchangers", Proceedings of the ASME Heat Transfer Division, v. 317-1, pp. 459-467.

Cengel, Y.A., Boles, M.A., 2002, “Thermodynamics - An Engineering Approach, $4^{\text {th }}$ Edition", McGraw-Hill.

Chisholm, D., 1985, “Two-Phase Flow in Heat Exchangers and Pipelines”, Heat Transfer Engineering, v. 6, n. 2, pp. 48-57.

Cole-Parmer Catalog, 2003-2004, “Compressors”, pp. 221-226.

De Andres, M.C., Hoo, E., Zangrando, F., 1995, "Performance of Direct-Contact Heat and Mass Exchangers with Steam-Gas Mixtures at Subatmospheric Pressures", Int'1 Journal of Heat and Mass Transfer, v. 39, n. 5, pp. 965-973. 
Deguerce, B., Banquet, F., Denisart, J.P., Favrat, D., 1984, "Use of Twin Screw Compressor for Steam Compression", $2^{\text {nd }}$ Int'l Symposium on the Large Scale Applications of Heat Pumps, York, England, pp. 189-196.

Devotta, S. Padalkar, A.S., Joshi, S.N., Sawant, N.N., and Sane, N.K., 2000, "Comparative assessment of $\mathrm{CO}_{2}$ for window air-conditioners", Proceedings of the $4^{\text {th }}$ IIR-Gustav Lorentzen Conference on Natural Working Fluids at Purdue.

Edwards, B.F., Wilder, J.W., Scime, E.E., 2001, "Dynamics of Falling Raindrops", European Journal of Physics, v. 22, pp. 113-118.

Electric Power Research Institute, 1998, "Electric Chiller Handbook".

El Wakil, M.M., 1984, “Powerplant Technology”, McGraw-Hill, Inc.

Fair, J.R., 1972, "Designing Direct-Contact Coolers/Condensers", Journal of Chemical Engineering, pp. 91-100.

Fletcher, L.S., Sernas, V., Galowin, L.S., 1974, "Evaporation from Thin Water Films on Horizontal Tubes", Ind. Eng. Chem, Process Des. Develop., v. 13, n.3, pp. 265-269.

Ford, J.D., Lekic, A., 1973, "Rate of Growth of Drops During Condensation", Int'l Journal of Heat and Mass Transfer, b.16, pp. 61-64.

Goodheart, K.A., 2000, “Low Firing Temperature Absorption Chiller System”, Master's Thesis, University of Wisconsin - Madison, WI.

Grazzini, G., D’Albero, M., 1998, “A Jet-Pump Inverse Cycle with Water Pumping Column", IIF/IIR - Oslo.

Honda, H., Takamatsu, H., Takata, N., 1999, "Experimental Measurements for Condensation of Downward Flowing R123/R-134a in a Staggered Bundle of Horizontal Low-finned Tubes with Four Fin Geometries", Int'1 Journal of Refrigeration, v. 22, pp. 615-624.

Hundy, G.F., Pham, H.M., 2001, "Effect of Refrigerant Choice on Efficiency in AirConditioning", Annual Conference of the Institute of Refrigeration, pp. 1-17.

Incropera, F.P., DeWitt, D.P., 2002, “Introduction to Heat Transfer, $4^{\text {th }}$ Edition”, John Wiley and Sons.

Kauffeld, M., Minds, G., Madsboll, H., 1997, "Water as Refrigerant”, LEGO Plant.

Kowalski, G.J., Loud, R.L., Kreith, F., "Experimental Investigation of a Direct-contact Heat Exchanger with Structured Packing". 
Kuhnl-Kinel, J., "New Age Water Chillers with Water as Refrigerant", CERN Geneva, Switzerland.

Lee, Y., Zeng, Y., Shigechi, T., 1989, "Conjugated Heat Transfer of Nucleate Pool Boiling on a Horizontal Tube", Int'1 Journal of Multiphase Flow, v. 16, n. 3, pp. 421-428.

Liu, J.P., 1975, “The Evaporating Falling Film on Horizontal Tubes”.

Liu, Z., Soedel, W., 1995, “A Mathematical Model for Simulating Liquid and Vapor Two-Phase Compression Process and Investigating Slugging Problems in Compressors", Journal of HVAC\&R Research, v. 1, n. 2, pp. 99-109.

Lund, S., 1998, "District Heat Assisted Ejector Cycle Refrigeration Plant for Process Cooling and Air-Conditioning Purposes”, IIF/IIR - pp. 71-80, Oslo.

Mudawar, I., Estes, K.A., 1996, "Optimizing and Predicting CHF in Spray Cooling of a Square Surface”, Journal of Heat Transfer, v. 118, pp. 672-679.

Müller, N., 2001, "Design of Compressor Impellers for Water as a Refrigerant", ASHRAE Transactions, Research, pp. 214-222.

Mussulman, R., Warrington, R., 1983, “Analysis of a liquid/gas direct-contact heat exchanger concept”, Journal of Energy, v 7, n 6, pp. 732-734.

Olivieri, J., Singh, T., 1996, "Psychrometrics: Theory and Practice", ASHRAE Publication, Research Project 872-RP.

Ortiz, M.G., Campo, A., Capozzi, V., Ochoa, D., Zinguer, M., 1982, "Direct-contact Heat Exchanger: An Experimental Study", Proceedings of the $7^{\text {th }}$ Int'l Heat Transfer Conference, Munchen, Germany, v. 6, pp. 337-340.

Owens, W.L., 1978, "Correlation of Thin Film Evaporation Heat Transfer Coefficients for Horizontal Tubes", Proceedings of the $5^{\text {th }}$ Ocean Thermal Energy Conversion Conference, v. 3, pp. VI71-VI89, Miami Beach.

Rao, D.N., Mohtadi, M.F., 1983, “A Novel Direct-contact Heat Exchange System for Efficient Heating of Water", ASME-JSME Thermal Engineering Joint Conf. v.2, pp. 113122, Honolulu.

Reindl, D.T., 1996, "Experimental Validation of Mathematical Models for Cooling by Direct-Contact Sprays", Proceedings from the EPRI International Sustainable Thermal Energy Storage Conference, Minneapolis, pp. 147-150, August.

Rice, W., 1981, "Calculated Characteristics of a Hydraulic Refrigeration System", Journal of Energy, v. 5, n. 6, pp. 323-330. 
Rini, D.P., Chen, R.H., Chow, L.C., 2002, "Bubble Behavior and Nucleate Boiling Heat Transfer in Saturated FC-72 Spray Cooling", Journal of Heat Transfer, v. 124, pp. 63-72.

Rohsenow, W.M., Hartnett, J.P., Cho, Y.I., 1998, "Handbook of Heat Transfer, 3 rd Edition".

Schick, R.J., Knasiak, K.F., 2000, "Spray Characterization For Wet Compression Gas Cooling Applications", $8^{\text {th }}$ Int'l Conference on Liquid Atomization and Spray Systems, California.

Schoonover, K.G., Ren, W.M., Ghiaasiaan, S.M., Abdel-Khalik, S.I., 1996, "Mechanistic Modeling of Desuperheater Performance", ISA Transactions, v. 35, pp. 45-51.

Sheer, T.J., Mitchley, S.R., 1998, "Vacuum Boiling in a Water Vapour Refrigeration System”, IIF/IIR - pp. 53-61, Oslo.

Sideman, S., Moalem, D., 1974, "Direct-contact Heat Exchangers: Comparison of Counter and Co-Current Condensers", Int'1 Journal of Multiphase Flow, v. 1, pp 555-572.

Stene, J., 1996, "Compression Systems with Natural Working Fluids", Proceedings of the $5^{\text {th }}$ Int'1 Energy Agency Conference on Heat Pumping Technologies, v. 1, pp. 215-222.

The Trane Company, 1965, "Trane Air Conditioning Manual”.

Tillner-Roth, R., Baehr, H.D., 1994, “An International Standard Formulation for the Thermodynamic Properties of 1,1,1,2-Tetrafluoroethane (HFC-134a) for Temperatures from $170 \mathrm{~K}$ to $455 \mathrm{~K}$ and Pressures up to $70 \mathrm{MPa}$,, J. Phys. Chem, Ref. Data, Vol. 23, No. 5.

Van Orshoven, D., 1991, "The use of water as a refrigerant - an exploratory investigation", Master's Thesis, University of Wisconsin - Madison, WI.

Van Orshoven, D., Klein, S.A., Beckman, W.A., 1993, “An Investigation of Water as a Refrigerant”, Journal of Energy Resources Technology, v. 115, pp. 257-263.

White, F.M., 1999, "Fluid Mechanics, $4^{\text {th }}$ Edition”, McGraw-Hill Co.

Wiesner, F.J., Caswell, H.E., 1959, "How Refrigerant Properties Affect Impeller Dimensions”, ASHRAE Journal, pp.31-37,104-106,114, October, 1959.

Wight, S.E., Tsukasa, Y., Le Drew, D’Orsi, N.C., B.A., 2000, “The Efficiency Limits of Water Vapor Compressors", Concepts ETI Final Report, Contract \# 605-10010.

Yuan, Q.S., Blaise, J.C., 1988, “Water - A Working Fluid for CFC Replacement”, Rev. Int. Froid, v. 11, pp. 243-247. 
Websites:

Bell and Gossett Pump Selection - www.bellgossett.com/selectpumps.stm

Brentwood Industries - www.brentwoodindustries.com/cool/cooll.htm

McMaster-Carr - www.mcmaster.com

Spraying Systems, Co. - www.spray.com

Wisconsin Public Service Corporation -

http://www.wisconsinpublicservice.com/business/wielecrate.asp 


\section{APPENDIX A: EES CODE}

\section{EES Code for four permutations of flash-intercooled water cycles:}

Note: Many variables are defined in Diagram Window, depicted at bottom.

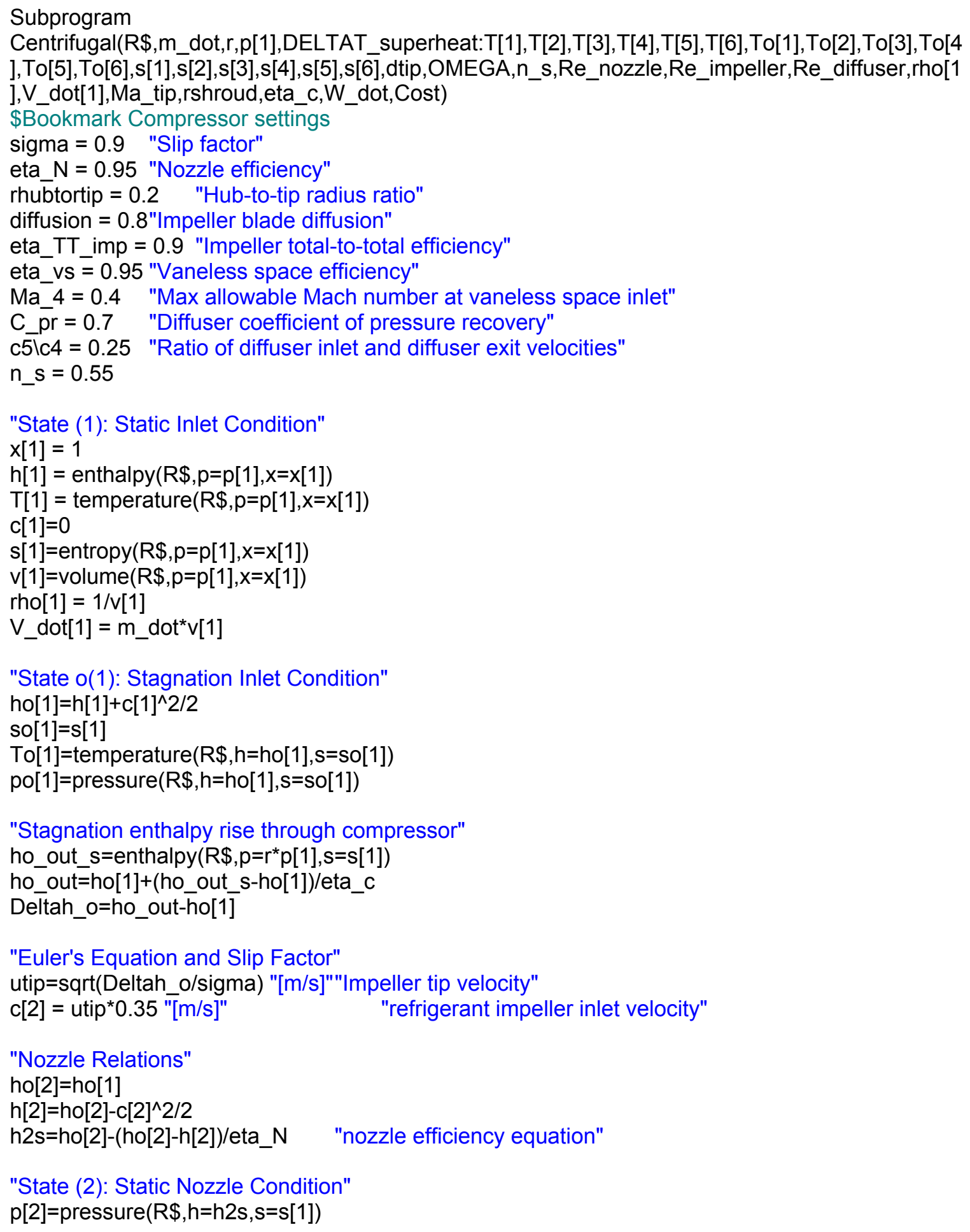


$\mathrm{s}[2]=\operatorname{entropy}(\mathrm{R} \$, \mathrm{p}=\mathrm{p}[2], \mathrm{h}=\mathrm{h}[2])$

$v[2]=$ volume $(R \$, p=p[2], h=h[2])$

$\mathrm{T}[2]=$ temperature $(\mathrm{R} \$, \mathrm{p}=\mathrm{p}[2], \mathrm{h}=\mathrm{h}[2])$

$x[2]=$ quality $(R \$, p=p[2], h=h[2])$

"State o(2): Stagnation Nozzle Condition"

$\mathrm{so}[2]=\mathrm{s}[2]$

To[2]=temperature $(\mathrm{R} \$, \mathrm{~h}=\mathrm{ho}[2], \mathrm{s}=\mathrm{so}[2])$

$\mathrm{Po}[2]=$ pressure $(\mathrm{R} \$, \mathrm{~h}=\mathrm{ho}[2], \mathrm{s}=\mathrm{so}[2])$

"Impeller Geometry"

OMEGA=n_s*Deltah_o^0.75/(m_dot* $\left.{ }^{*}[1]\right)^{\wedge} 0.5$ "Impeller rotational velocity"

rtip=utip/omega

dtip $=2^{*}$ rtip

rhub $=$ rhubtortip*rtip

rshroud=sqrt(rhub^2+m_dot* $v[2] /\left(c[2]^{*}\right.$ pi $\left.)\right)$

"Geometric constraint of hub-to-tip ratio"

"Mass conservation"

"Blade entrance velocities"

u_hub = omega*rhub

"kinematic relationship between rotational speed

and impeller speed"

u_shroud = omega ${ }^{*}$ rshroud

"Calculate the hub and shroud blade angles"

Beta_hub $=\arctan \left(\right.$ u_hub/c[2]) ${ }^{*}$ convert(rad,deg)

"from velocity vector diagrams"

Beta_shroud $=\arctan (\text { u_shroud/c[2] })^{\star}$ convert $($ rad,deg $)$

"Determine the relative velocity at the inlet shroud

and the relative Mach number at the inlet shroud"

w_shroud $=\operatorname{sqrt}\left(u \_s h r o u d^{\wedge} 2+c[2]^{\wedge} 2\right)$

Ma_r_shroud $=w \_s h r o u d /$ soundspeed $(R \$, T=T[2], P=P[2])$

Ma_shroud $=c[2] /$ soundspeed $(R \$, T=T[2], P=P[2])$

"Impeller Blade diffusion"

w_tip $=$ diffusion ${ }^{*} w \_$shroud

"diffusion of fluid momentum across impeller

blade"

c_slip $=(1 \text {-sigma })^{*}$ utip

c[3]=sqrt((w_tip^2-c_slip^2)+utip^2*sigma^2)

"from velocity vector diagrams"

"State o(3): Impeller Exit Stagnation State"

ho3s=ho[2]+eta_TT_imp*(ho[3]-ho[2])

"impeller efficiency equation"

$\mathrm{Po}[3]=$ pressure $(\mathrm{R} \$, \overline{\mathrm{s}}=\mathrm{so}[2], \mathrm{h}=\mathrm{ho} 3 \mathrm{~s})$

To[3] =temperature $(\mathrm{R} \$, \mathrm{P}=\mathrm{Po}[3], \mathrm{h}=\mathrm{ho}[3])$

ho[3]=ho_out

so[3]=entropy $(\mathrm{R} \$, \mathrm{P}=\mathrm{Po}[3], \mathrm{h}=\mathrm{ho}[3])$

"State (3): Impeller Exit Static State"

$\mathrm{h}[3]=\mathrm{ho}[3]-\mathrm{c}[3]^{\wedge} 2 / 2$

$\mathrm{s}[3]=\mathrm{so}[3]$

$\mathrm{T}[3]=$ temperature $(\mathrm{R} \$, \mathrm{~h}=\mathrm{h}[3], \mathrm{s}=\mathrm{s}[3])$

$v[3]=$ volume $(R \$, h=h[3], s=s[3])$

$P[3]=$ pressure $(R \$, h=h[3], s=s[3])$

"Tip Speed Checks"

Ma_tip $=c[3] / S O U N D S P E E D(R \$, T=T[3], p=p[3])$

Ma_r_tip=w_tip/soundspeed $(R \$, T=T[3], p=p[3])$

"Conservation of angular momentum in vaneless space" 


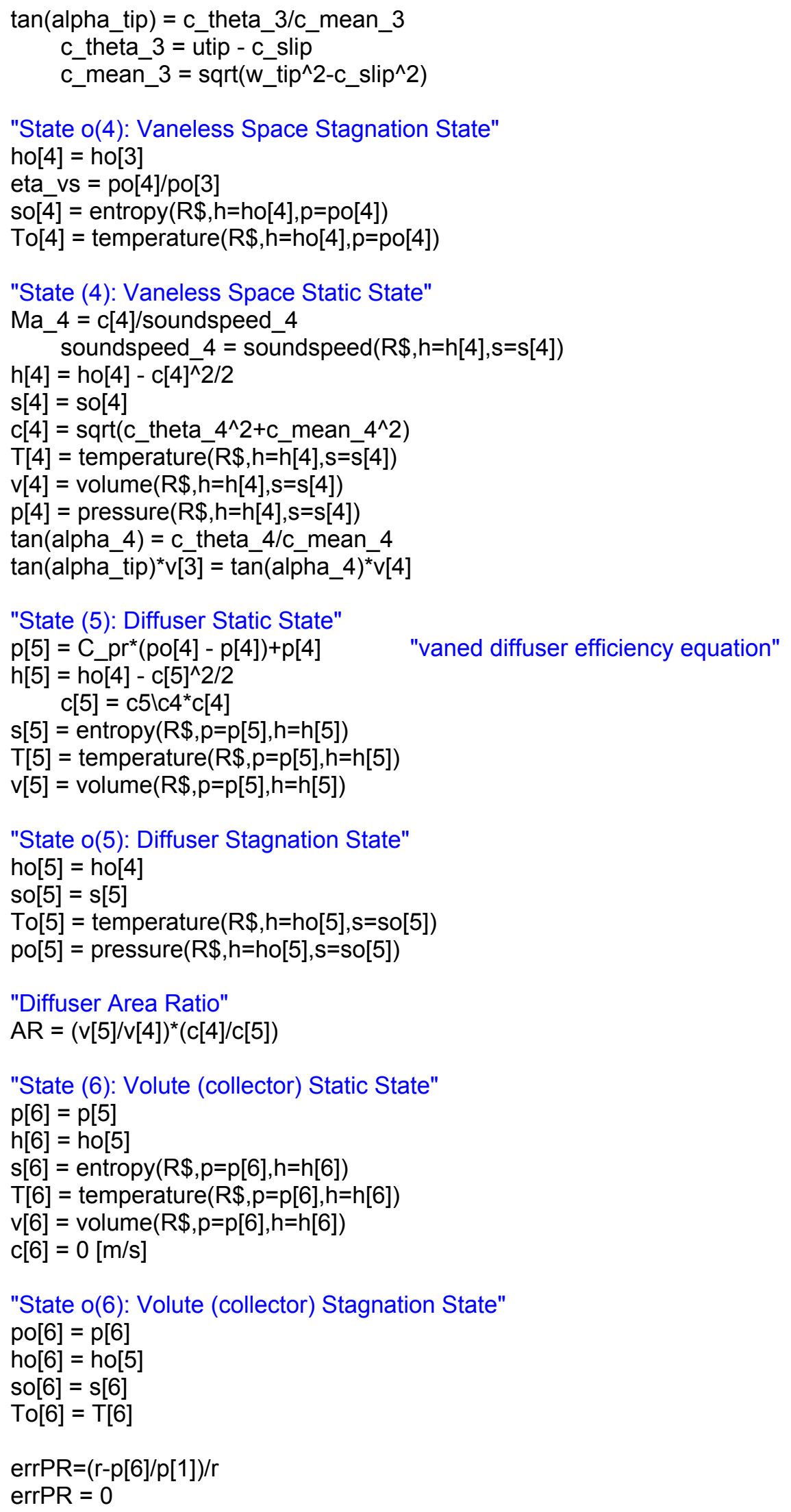




$$
\begin{aligned}
& \mathrm{s}[4]=\text { entropy }(\mathrm{R} \$, T=T[4], p=p[4]) \\
& \mathrm{v}[4]=\text { volume }(\mathrm{R} \$, T=T[4], p=p[4])
\end{aligned}
$$

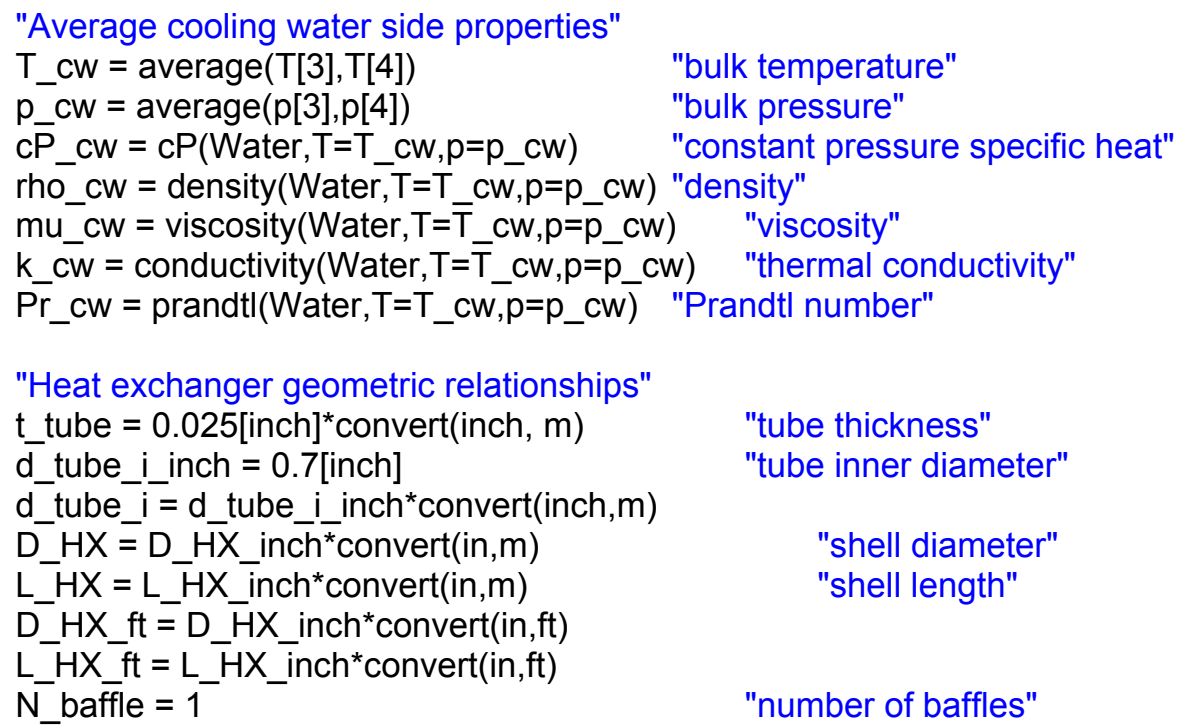




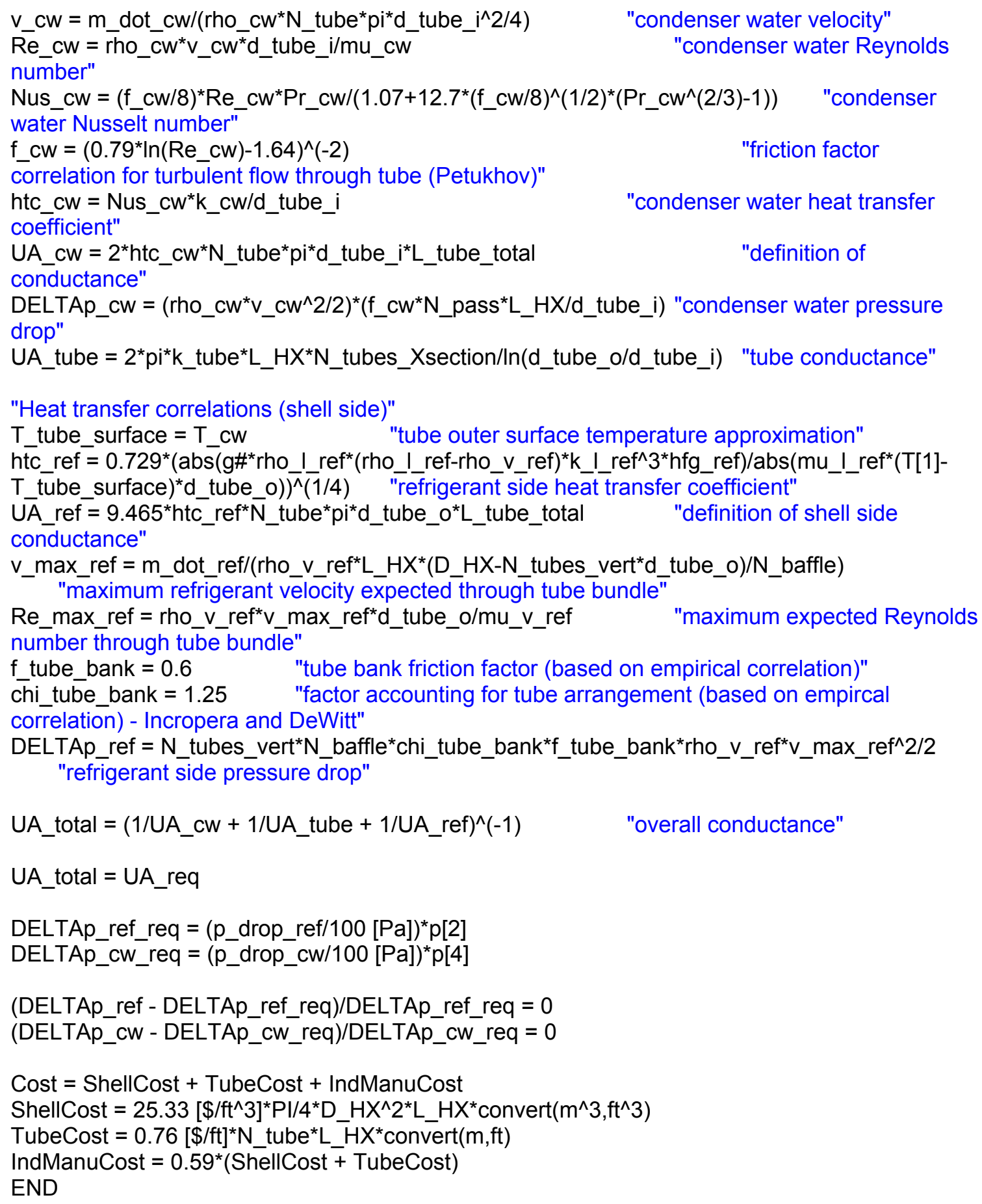


"State 1: Refrigerant Inlet"

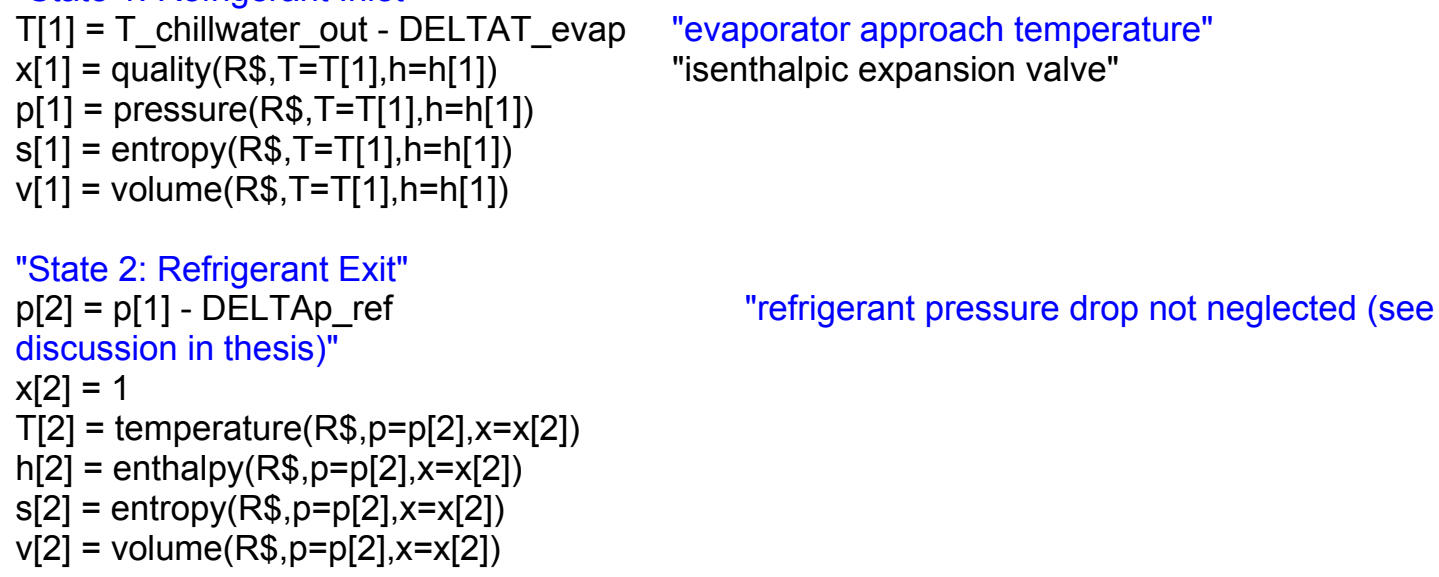

"State 3: Chilled Water Inlet"
$\mathrm{T}[3]=\mathrm{T}$ _chillwater_in $\quad$ "chilled water inlet temperature"
$p[3]=\left(1+p \_d r o p \_c h w / 100[P a]\right)^{*} p_{-}$atm $\quad$ "chilled water pressure drop"
$\mathrm{h}[3]=$ enthalpy $($ Water, $\mathrm{T}=\mathrm{T}[3], \mathrm{p}=\mathrm{p}[\overline{3}])$
$\mathrm{s}[3]=$ entropy $($ Water, $T=T[3], p=p[3])$
$\mathrm{v}[3]=$ volume $($ Water $, T=T[3], p=p[3])$
$\mathrm{x}[3]=$ quality $($ Water $, \mathrm{T}=\mathrm{T}[3], \mathrm{p}=\mathrm{p}[3])$

"State 4: Chilled Water Exit"

$\mathrm{T}[4]=\mathrm{T}$ _chillwater_out $\quad$ "chilled water exit temperature"

$\mathrm{p}[4]=\mathrm{p} \_$atm

$\mathrm{h}[4]=$ enthalpy $($ Water, $\mathrm{p}=\mathrm{p}[4], \mathrm{T}=\mathrm{T}[4])$

$\mathrm{s}[4]=$ entropy $($ Water, $\mathrm{p}=\mathrm{p}[4], \mathrm{T}=\mathrm{T}[4])$

$\mathrm{v}[4]=$ volume $($ Water $, p=p[4], T=T[4])$

$x[4]=$ quality $($ Water, $p=p[4], T=T[4])$

"Chilled water properties"

p_chw $=p[4]$

T_chw $=$ average $(\mathrm{T}[3], \mathrm{T}[4])$

"bulk pressure"

C_p_chw $=c P\left(\right.$ 'Water', $\left.p=p \_c h w, T=T \_c h w\right)$ "bulk temperature"

Rho_chw $=$ density('Water', $p=p \_c h w, T=T$ chw) "density"

mu_chw = viscosity('Water', $p=p_{-}$chw, $T=T_{-}$chw) "viscosity"

k_ch $w=$ conductivity('Water', $p=p_{-} \_$chw, $\left.T=\bar{T} \_c h w\right) \quad$ "thermal conductivity"

Pr_chw $=$ prandtl('Water', $\left.p=p_{-} \_c h \bar{w}, T=T \_c h \bar{w}\right) \quad$ "Prandtl number"

"Heat exchanger geometry"

t_tube $=0.025[\text { inch }]^{*}$ convert(inch, $\mathrm{m}$ )

d_tube_i_inch $=0.7[$ inch]

"tube thickness"

d_tube_i $=$ d_tube_i_inch* convert(inch, $m$ )

D_HX = D_HX_inch ${ }^{*}$ convert(inch, $m$ ) "shell diameter"

$L_{-} H X=L \_$HX inch*onvert(inch, $m$ )

D_HX_ft $=$ D_HX_inch*onvert(inch, ft $)$

$\mathrm{L}_{-} \mathrm{HX}_{-} \mathrm{ft}=\mathrm{L}_{-} \mathrm{H} X{ }_{-}$inch ${ }^{*}$ convert (inch, $\mathrm{ft}$ )

N_baffle $=1$

"number of baffles"

"Tube Properties"

k_tube = k_(T\$,converttemp $(\mathrm{C}, \mathrm{K},(\mathrm{T}[3]+\mathrm{T}[4]) / 2))$ "tube thermal conductivity"

"Refrigerant properties"

rho_I_ref $=\operatorname{density}(\mathrm{R} \$, \mathrm{~T}=\mathrm{T}[2], \mathrm{x}=0) \quad$ "liquid refrigerant density" 


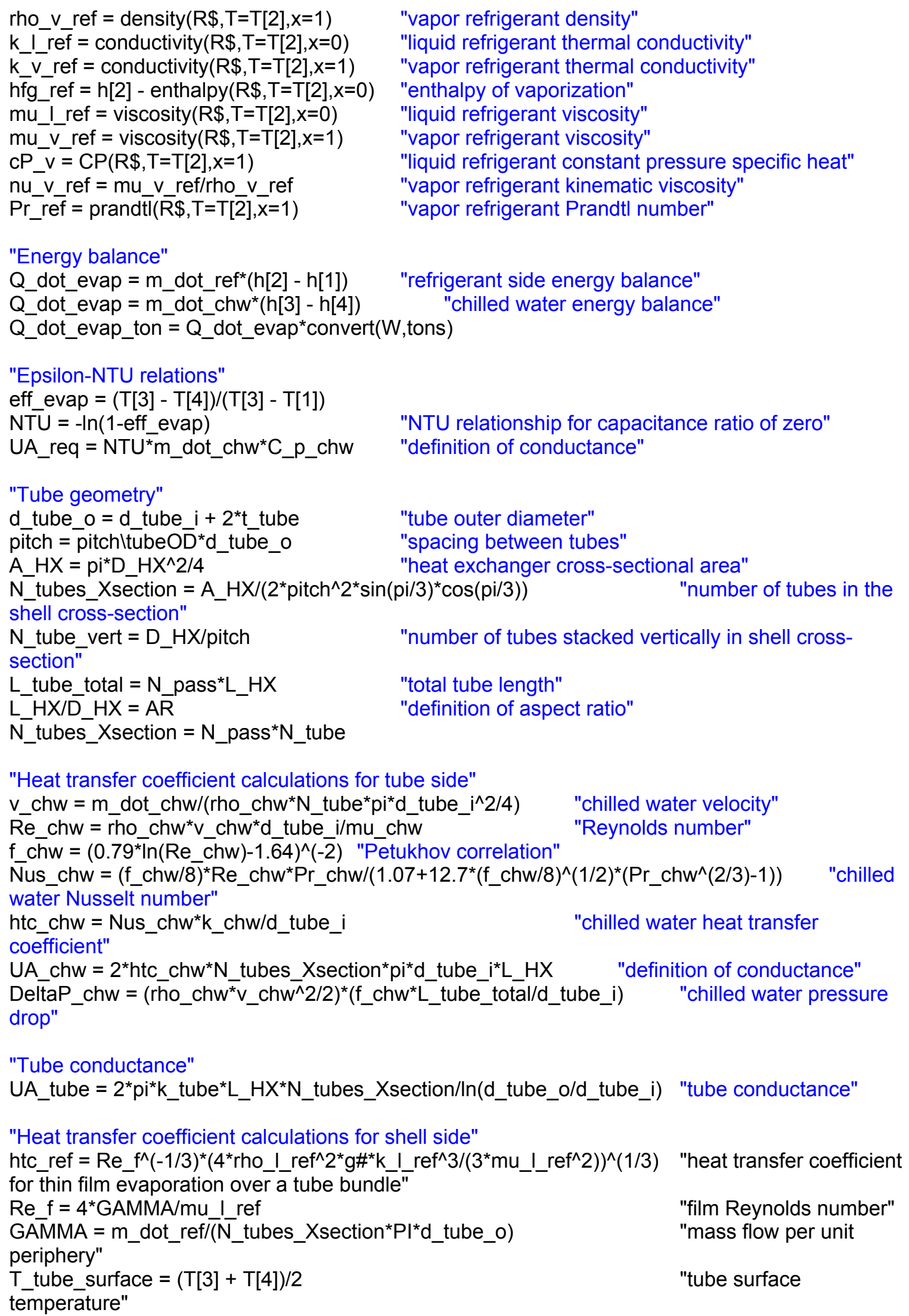




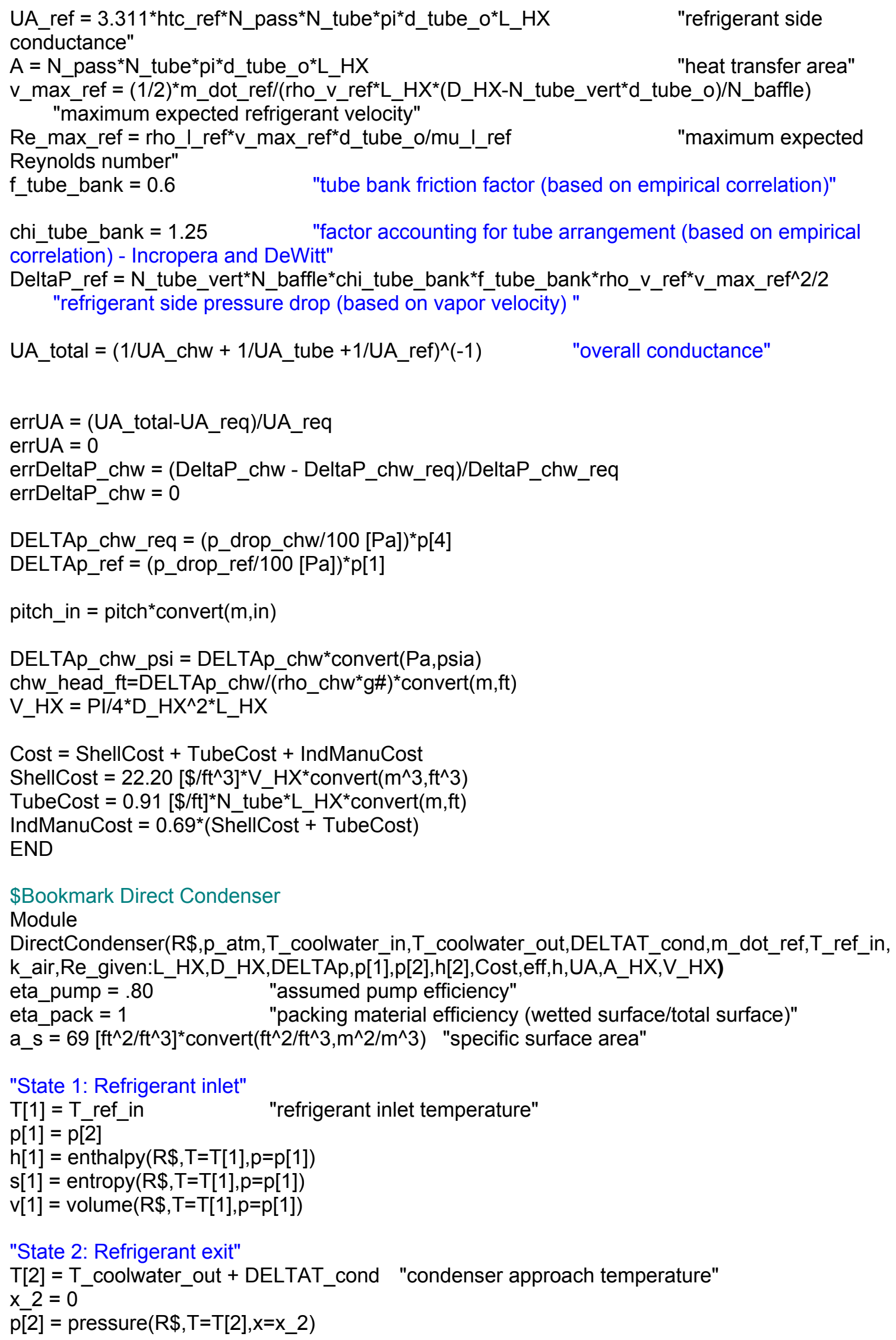

"State 2: Refrigerant exit"

$\mathrm{T}[2]=\mathrm{T}$ _coolwater_out + DELTAT_cond "condenser approach temperature" $x+2=0$

$\mathrm{p}[2]=$ pressure $\left(\mathrm{R} \$, \mathrm{~T}=\mathrm{T}[2], \mathrm{x}=\mathrm{x} \_2\right)$ 


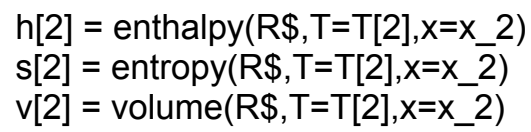

"State 3: Cooling water inlet"

$\mathrm{T}[3]=\mathrm{T}$ _coolwater_in $\quad$ "condenser water inlet temperature"

$\mathrm{p}[3]=\mathrm{p} \_$atm

$\mathrm{h}[3]=$ enthalpy $(\mathrm{R} \$, \mathrm{~T}=\mathrm{T}[3], \mathrm{p}=\mathrm{p}[3])$

$\mathrm{s}[3]=\operatorname{entropy}(R \$, T=T[3], p=p[3])$

$v[3]=$ volume $(R \$, T=T[3], p=p[3])$

"State 4: Cooling water after expansion valve"

$\mathrm{p}[4]=\mathrm{p}[1]$

$\mathrm{h}[4]=\mathrm{h}[3]$

$\mathrm{T}[4]=$ temperature $(\mathrm{R} \$, \mathrm{p}=\mathrm{p}[4], \mathrm{h}=\mathrm{h}[4])$

$\mathrm{s}[4]=$ entropy $(R \$, p=p[4], h=h[4])$

$v[4]=\operatorname{volume}(R \$, p=p[4], h=h[4])$

"State 5: Cooling water before pump"

$\mathrm{p}[5]=\mathrm{p}[2]$

eta_pump $=\left(h \_6 s-h[5]\right) /(h[6]-h[5])$ "pump efficiency"

$\mathrm{T}[5]=$ temperature $(R \$, p=p[5], h=h[5])$

$s[5]=$ entropy $(R \$, p=p[5], h=h[5])$

$v[5]=\operatorname{volume}(R \$, p=p[5], h=h[5])$

"State 6: Cooling water exit"

$\mathrm{p}[6]=\mathrm{p} \_$atm

$\mathrm{T}[6]=\mathrm{T}$ _coolwater_out

$h[6]=$ enthalpy $(R \$, \bar{T}=T[6], p=p[6])$

"condenser water exit temperature"

$s[6]=\operatorname{entropy}(R \$, T=T[6], p=p[6])$

$v[6]=$ volume $(R \$, T=T[6], p=p[6])$

$h \_6 s=$ enthalpy $(R \$, p=p[6], s=s[5])$

"Energy Balance"

$\mathrm{m}$ _dot_cw $=\mathrm{m}$ _dot_ref* $(\mathrm{h}[1]-\mathrm{h}[2]) /(\mathrm{h}[5]-\mathrm{h}[4])$ "condenser water side energy balance"

$\mathrm{q}=\mathrm{m} \_$dot_ref* $(\mathrm{h}[1]-\mathrm{h}[2])$

"refrigerant side energy balance"

"Effectiveness-NTU method"

$\mathrm{C} \_\mathrm{w}=\mathrm{m} \_$dot_cw* $\mathrm{CP}($ Water, $\mathrm{T}=\mathrm{T}[4], \mathrm{p}=\mathrm{p}[4])$

$\mathrm{Eff}=(\mathrm{T}[\overline{5}]-\mathrm{T}[3]) /(\mathrm{T}[2]-\mathrm{T}[3])$

NTU $=-\ln (1-$ eff $)$

"condenser water heat capacity"

"definition of effectiveness"

NTU $=$ UA/C_w "definition of NTU for capacitance ratio of zero" "definition of NTU"

rho_I = density $(\mathrm{R} \$, \mathrm{~T}=\mathrm{T}[2], \mathrm{x}=0) \quad$ "liquid refrigerant density"

rho_ $v=\operatorname{density}(\mathrm{R} \$, \mathrm{~T}=\mathrm{T}[2], \mathrm{x}=1) \quad$ "vapor refrigerant density"

$\mathrm{k} \_\mathrm{I}=$ conductivity $(\mathrm{R} \$, \mathrm{~T}=\mathrm{T}[2], \mathrm{x}=0) \quad$ "liquid refrigerant thermal conductivity"

$\mathrm{hfg}=$ enthalpy $(\mathrm{R} \$, \mathrm{~T}=\mathrm{T}[2], \mathrm{x}=1)-\mathrm{h}[2] \quad$ "enthalpy of vaporization"

$\mathrm{cP} \_\mathrm{I}=\mathrm{CP}(\mathrm{R} \$, \mathrm{~T}=\mathrm{T}[2], \mathrm{x}=0) \quad$ "liquid refrigerant constant pressure specific heat"

T_sat $=\mathrm{T}[2] \quad$ "refrigerant saturation temperature"

T_s $=(T$ _coolwater_in + T_coolwater_out $) / 2$ "average condenser water temperature"

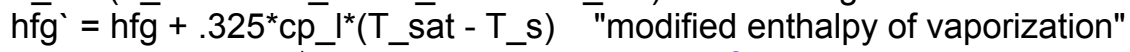

mu_I = viscosity $(R \$, \bar{T}=T[\overline{2}], x=0) \quad$ "liquid refrigerant viscosity"

mu_v $=$ viscosity $(R \$, T=T[2], x=1) \quad$ "vapor refrigerant viscosity"

$\mathrm{AR}=\mathrm{L}_{-} \mathrm{HX} / \mathrm{D}_{-} \mathrm{HX} \quad$ "definition of aspect ratio"

L_side $=0.01 \overline{2}[\mathrm{~m}] \quad$ "length of corrugation" 


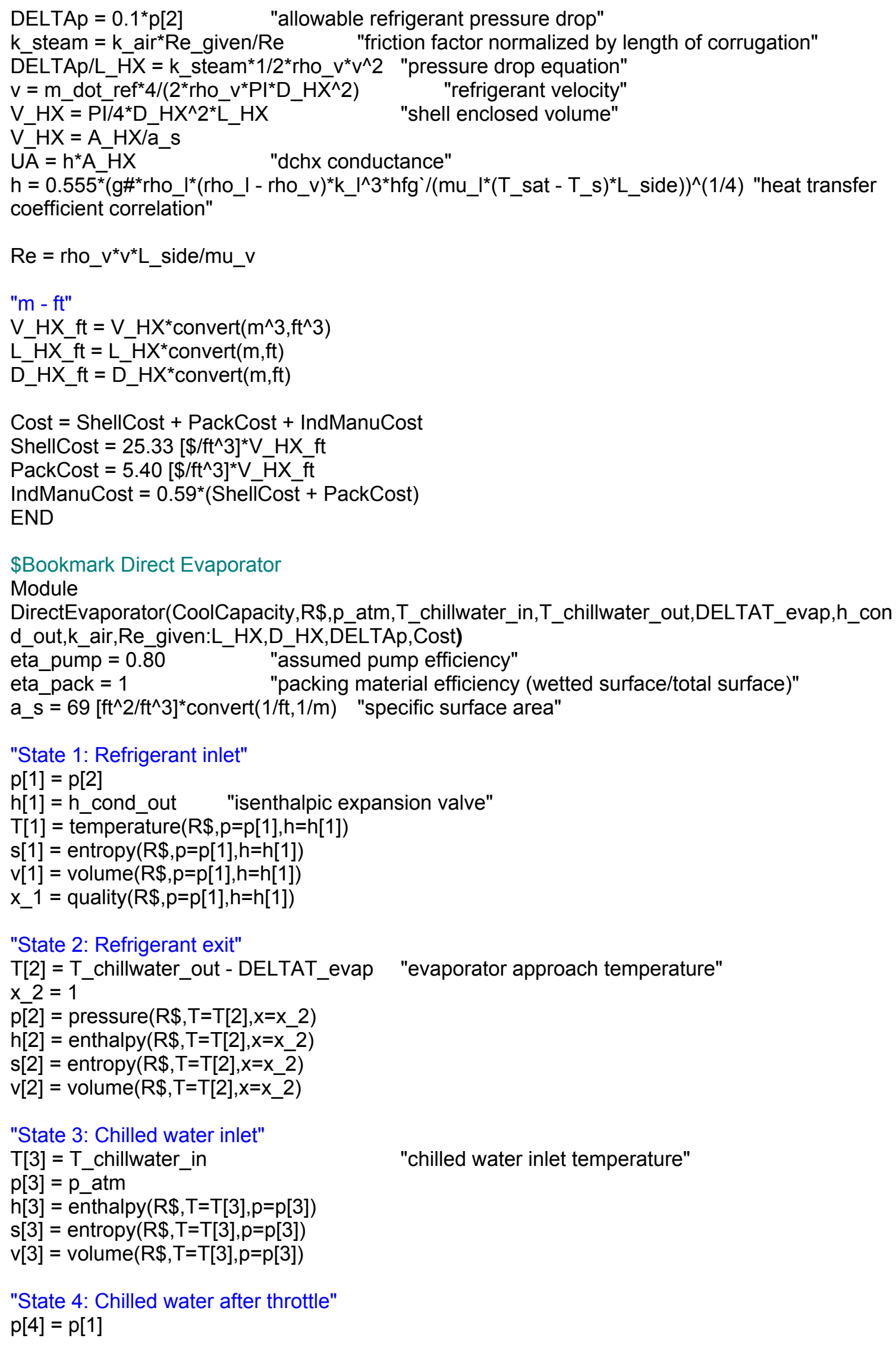

"State 2: Refrigerant exit"

T[2] = T_chillwater_out - DELTAT_evap "evaporator approach temperature"

$\times 2=1$

$\mathrm{p}[2]=$ pressure $\left(\mathrm{R} \$, \mathrm{~T}=\mathrm{T}[2], \mathrm{x}=\mathrm{x} \_2\right)$

$\mathrm{h}[2]=$ enthalpy $\left(\mathrm{R} \$, \mathrm{~T}=\mathrm{T}[2], \mathrm{x}=\mathrm{x} \_2\right)$

$\mathrm{s}[2]=$ entropy $\left(\mathrm{R} \$, T=T[2], \mathrm{x}=\mathrm{x} \_\right.$2 $)$

$\mathrm{v}[2]=$ volume $\left(\mathrm{R} \$, \mathrm{~T}=\mathrm{T}[2], \mathrm{x}=\mathrm{x} \_2\right)$

"State 3: Chilled water inlet"

$\mathrm{T}[3]=\mathrm{T}$ _chillwater_in

"chilled water inlet temperature"

$\mathrm{p}[3]=\mathrm{p} \_$atm

$\mathrm{h}[3]=$ enthalpy $(\mathrm{R} \$, \mathrm{~T}=\mathrm{T}[3], \mathrm{p}=\mathrm{p}[3])$

$s[3]=\operatorname{entropy}(R \$, T=T[3], p=p[3])$

$v[3]=$ volume $(R \$, T=T[3], p=p[3])$

"State 4: Chilled water after throttle" $\mathrm{p}[4]=\mathrm{p}[1]$ 


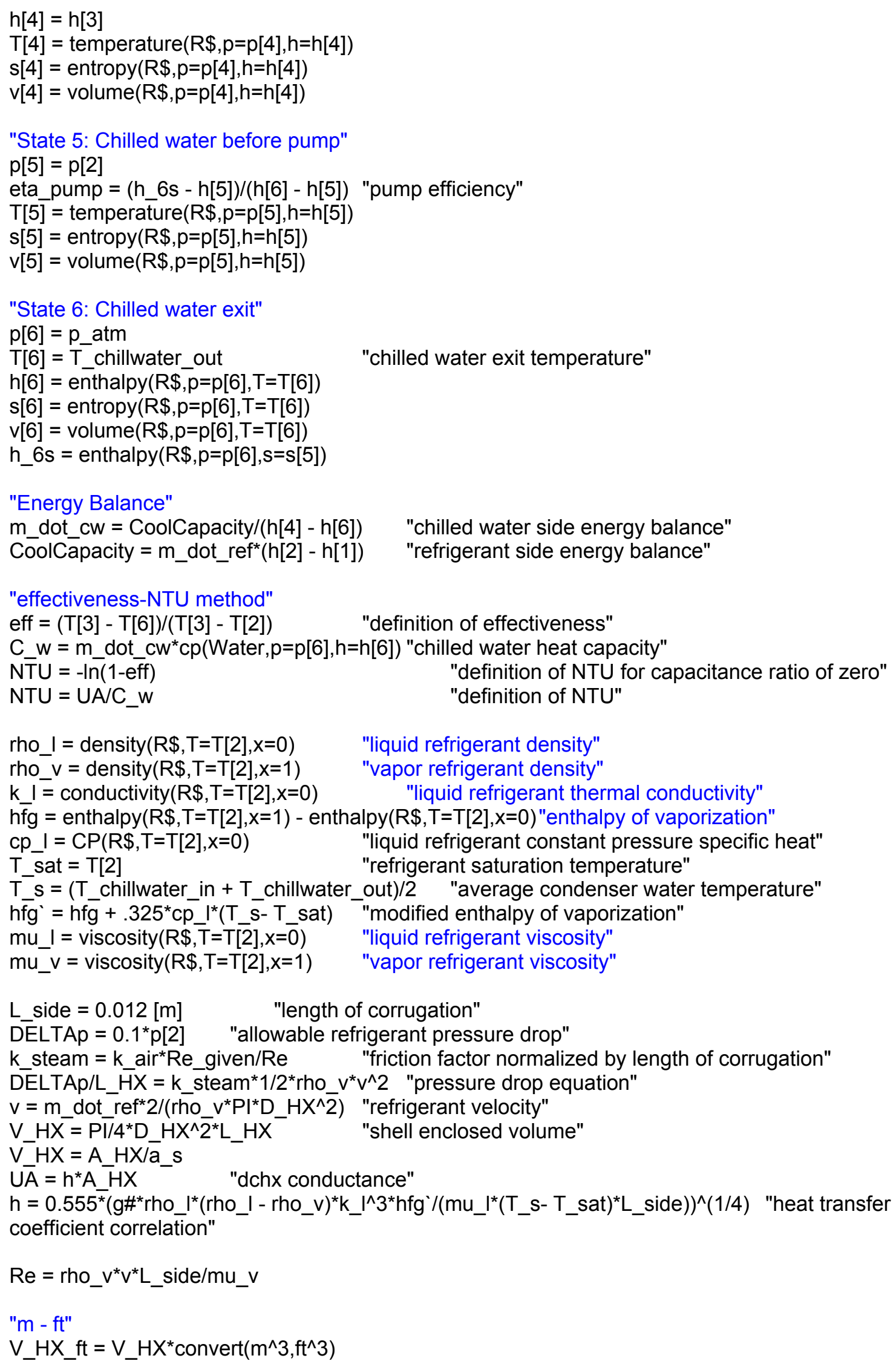




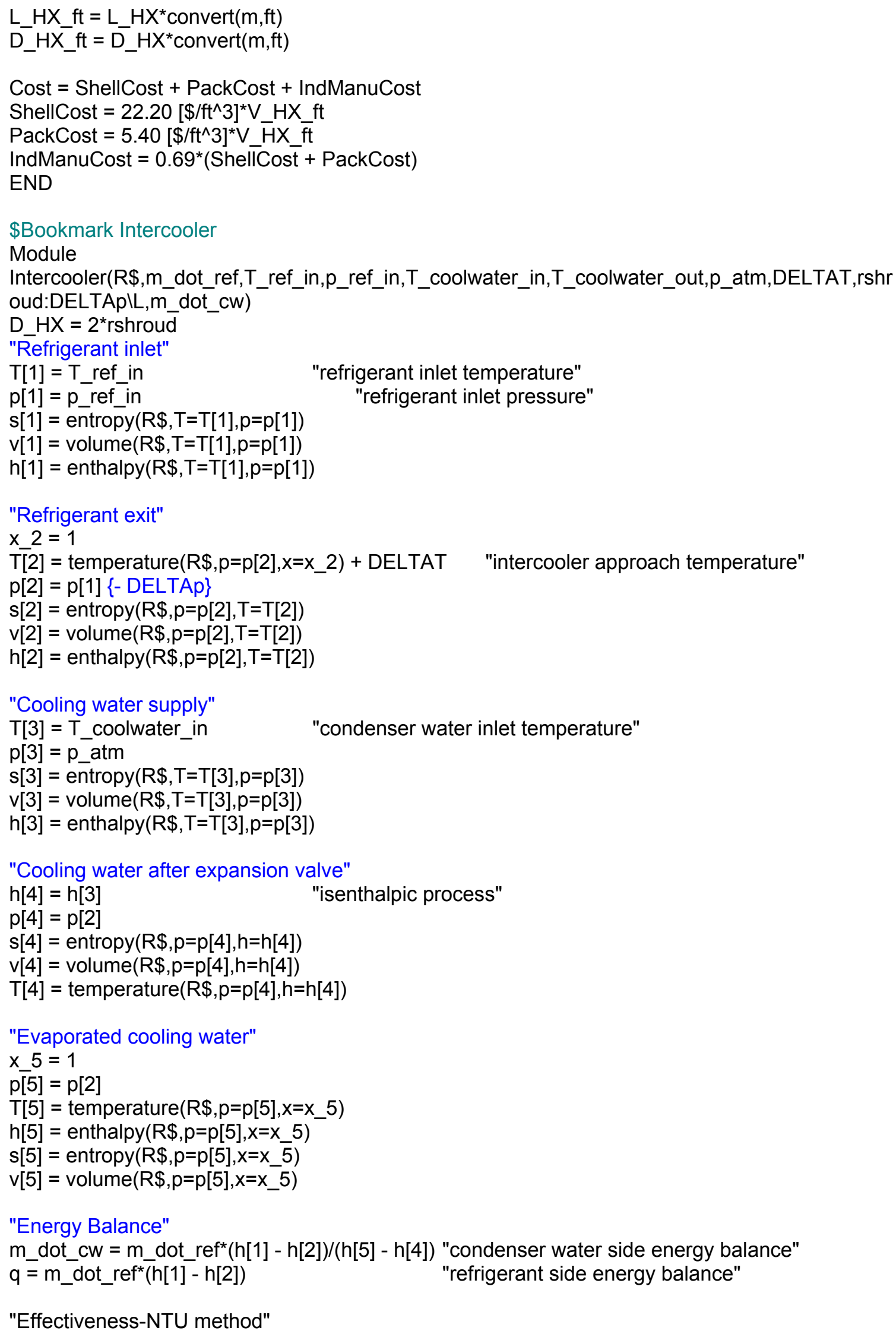

"Cooling water after expansion valve" 


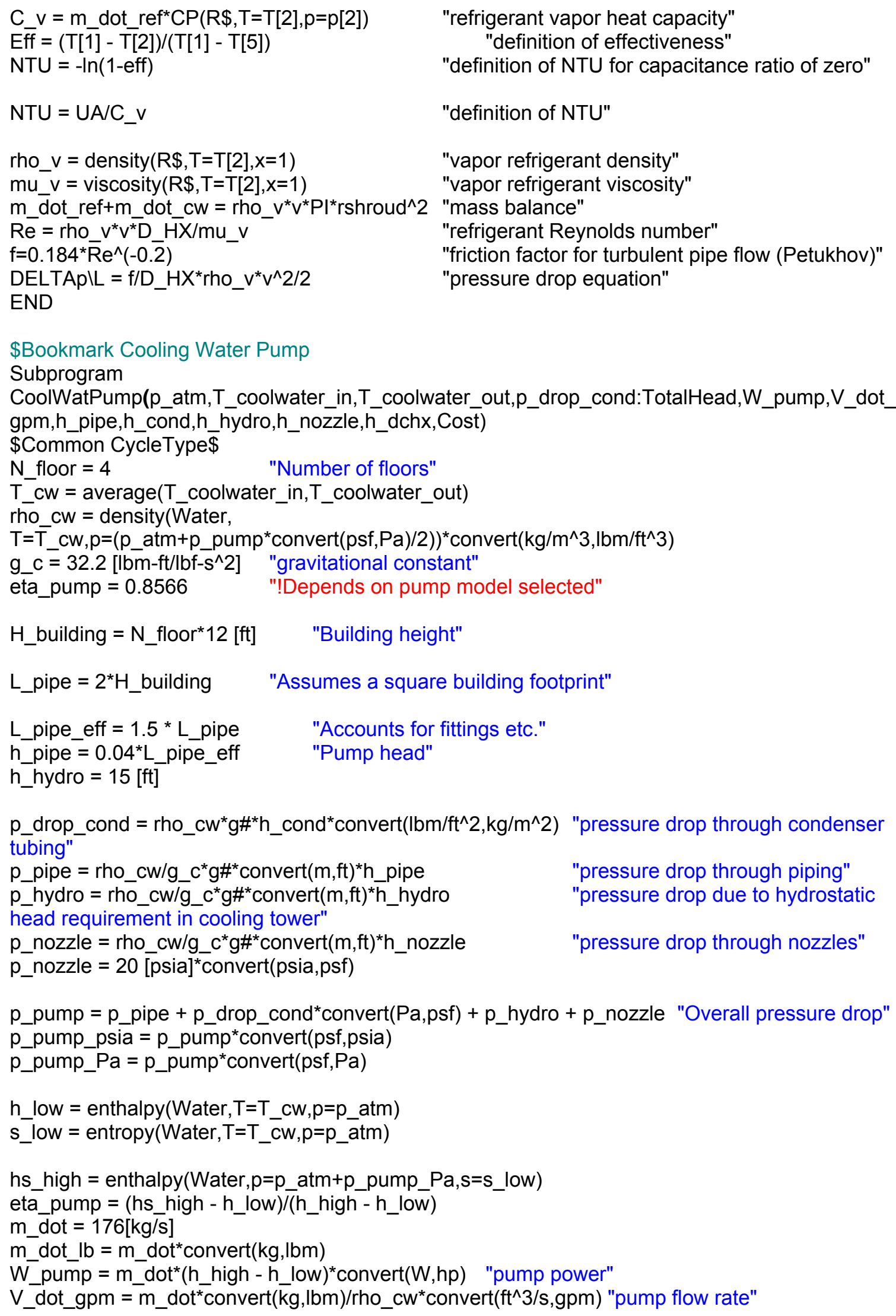




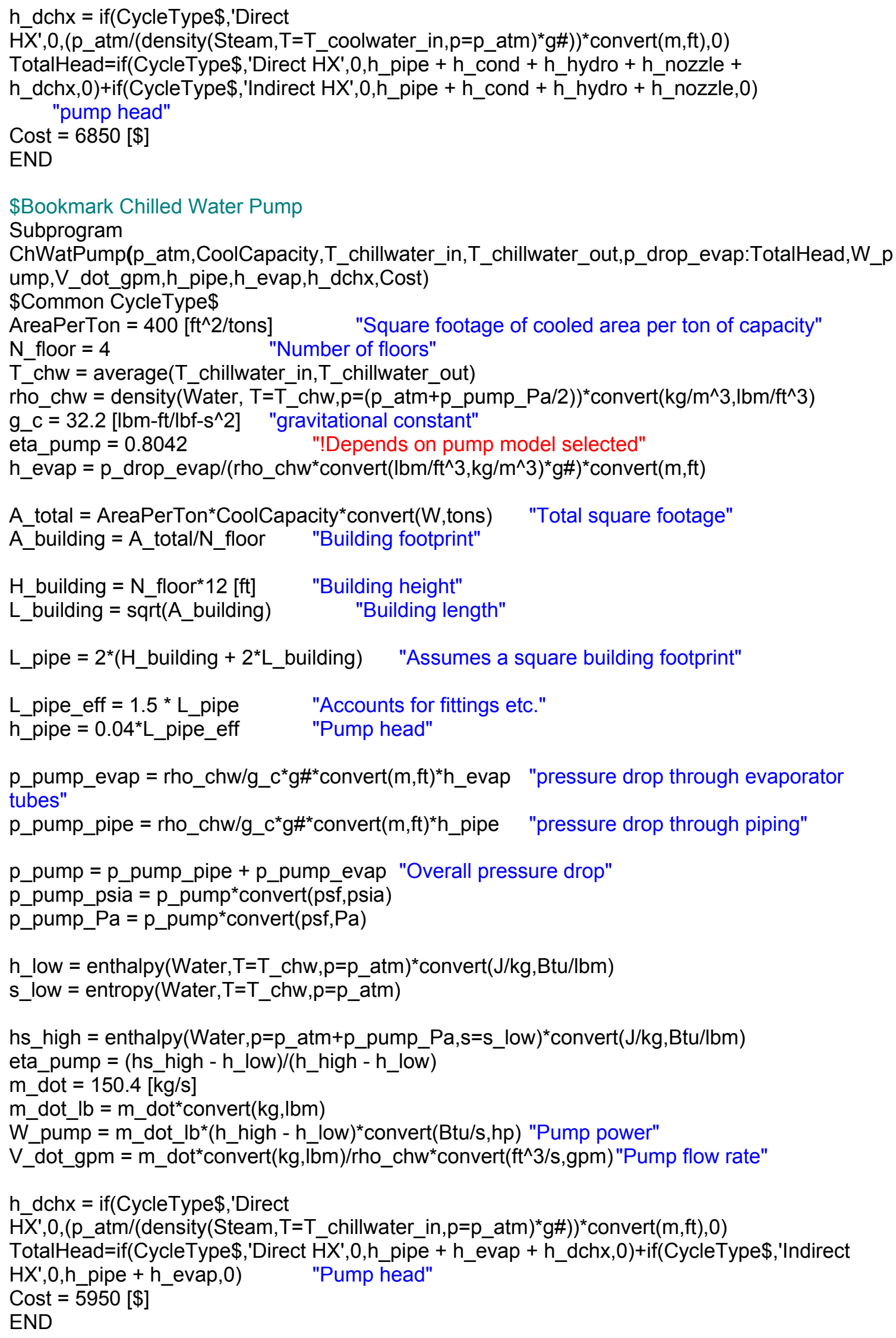


\$Bookmark Cost Analysis

Subprogram

CostAnalysis(City\$,EnergyCost,W_dot,CompCost,EvapCost,CondCost,PumpCost,HrsYr,n_yr:LC $\mathrm{C}, \mathrm{OC}, \mathrm{E})$

TCC $=$ CompCost + CondCost + EvapCost + PumpCost

"Economic parameters"

$\mathrm{DP}=0.20 \quad$ "ratio of down payment to initial investment"

$\mathrm{f}=0.05 \quad$ "fuel inflation rate"

$\mathrm{d}=0.05 \quad$ "market discount rate"

$\mathrm{m}=0.075 \quad$ "annual mortgage interest rate"

$\mathrm{i}=0.02 \quad$ "general inflation rate"

$\mathrm{N}=20 \quad$ "period of economic analysis"

$\mathrm{N} L=20 \quad$ "term of loan"

$\mathrm{N}_{-} 1=\mathrm{MIN}\left(\mathrm{N}, \mathrm{N} \_\mathrm{L}\right) \quad$ "years over which mortgage payment scontribute to the analysis (min of $\mathrm{N}$ or N_L)"

N_D = $39 \quad$ "depreciation lifetime in years"

N_2 = MIN (N,N_D) "years over which depreciation contributes to the analysis ( $\min$ of $\mathrm{N}$ or

N-D)"

$\mathrm{p}=0.035 \quad$ "property tax rate based on assessed value"

$\mathrm{c}=1 \quad$ "either 1 for commerical investment or 0 for a residential investment"

$\mathrm{t}=0.40 \quad$ "effective income tax rate"

$S=0.20 \quad$ "ratio of resale value at end of period of analysis to initial investment"

$\mathrm{OC}=$ EnergyCost ${ }^{*} \mathrm{~W} \_$dot ${ }^{*} \mathrm{Hrs} Y \mathrm{r} \quad$ "First year operating cost"

$\mathrm{E}=\mathrm{TCC} \quad$ "First cost of the system"

$\mathrm{LCC}=\mathrm{P} \_1^{*} \mathrm{OC}+\mathrm{P} 2^{*} \mathrm{E}$

$P \_1=(1-t)^{*} P W \overline{F F}(N, f, d)$

P_2 $=$ DP + (1-DP)* $\left(P W F\left(N \_1,0, d\right) / P W F\left(N \_L, 0, m\right)\right)-t^{*}(1-D P)^{*}\left(P W F\left(N \_1, m, d\right)^{*}(m-\right.$

1/PWF(N_L,0,m) $\left.)+P W F\left(N \_1,0, d\right) / P W F\left(N \_L, 0, m\right)\right)+\left(p^{*}(1-t)+M^{*}\left(1-c^{*} t\right)\right)^{*} P W F(N, i, d)-$

$C^{*} t / N \_D^{*} P W F\left(N \_2,0, d\right)-S-t$

END

\$Bookmark Main Program

$\mathrm{R} \$=$ 'Steam'

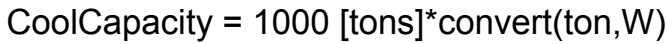

\{ARI Conditions\}

T_chillwater_in $=$ CONVERTTEMP $(F, C, 54)$

T_chillwater_out $=$ CONVERTTEMP $(F, C, 44) \quad$ "chilled water exit temperature"

$\mathrm{T}_{\text {_coolwater in }}$ = CONVERTTEMP $(\mathrm{F}, \mathrm{C}, 85)$ "condenser water inlet temperature"

T_coolwater_out $=$ CONVERTTEMP $(F, C, 95)$ "condenser water exit temperature"

DELTAT_cond = DELTAT_cond_F*convert $(F, C) \quad$ "Condenser approach temperature"

DELTAT_evap $=$ DELTAT_evap_F*convert $(F, C) \quad$ "Evaporator approach temperature"

DELTAT_superheat $=1[\bar{F}]^{*}$ convert $(F, C)$

DELTAT_int $=$ DELTAT_cond

DELTAT_int_F = DELTATT_cond_F

N_stages $=\overline{2} \quad$ "number of compression stages"

"Set up guess values (help EES iteration loop - these equations have no bearing on solution other than that it helps EES converge)"

$\mathrm{Tg}=16[\mathrm{C}]$

$\mathrm{Pg}=$ pressure $(\mathrm{R} \$, \mathrm{~T}=\mathrm{Tg}, \mathrm{x}=1)^{*} 0.8$

$\mathrm{vg}=$ volume $(\mathrm{R} \$, \mathrm{~T}=\mathrm{Tg}, \mathrm{P}=\mathrm{Pg})$

sg=entropy $(\mathrm{R} \$, \mathrm{~T}=\mathrm{Tg}, \mathrm{P}=\mathrm{Pg})$

$\mathrm{hg}=$ enthalpy $(\mathrm{R} \$, \mathrm{~T}=\mathrm{Tg}, \mathrm{P}=\mathrm{Pg})$

soundspeedg $=$ soundspeed $(R \$, T=T g, p=P g)$ 


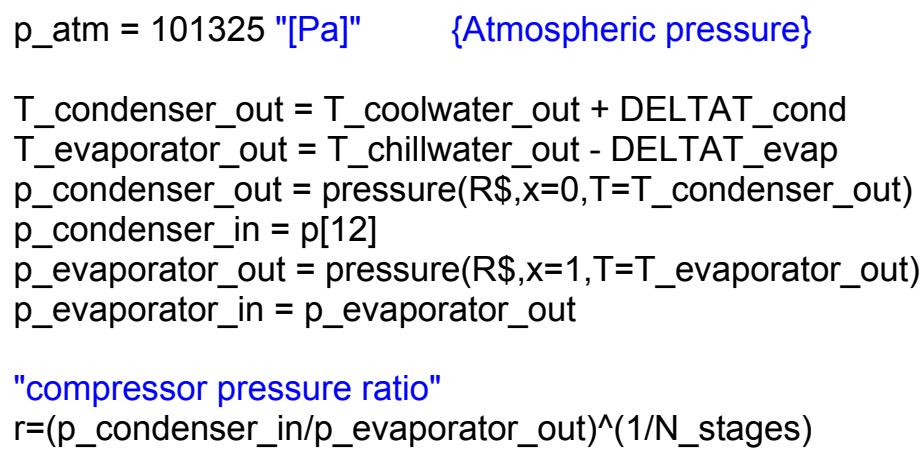




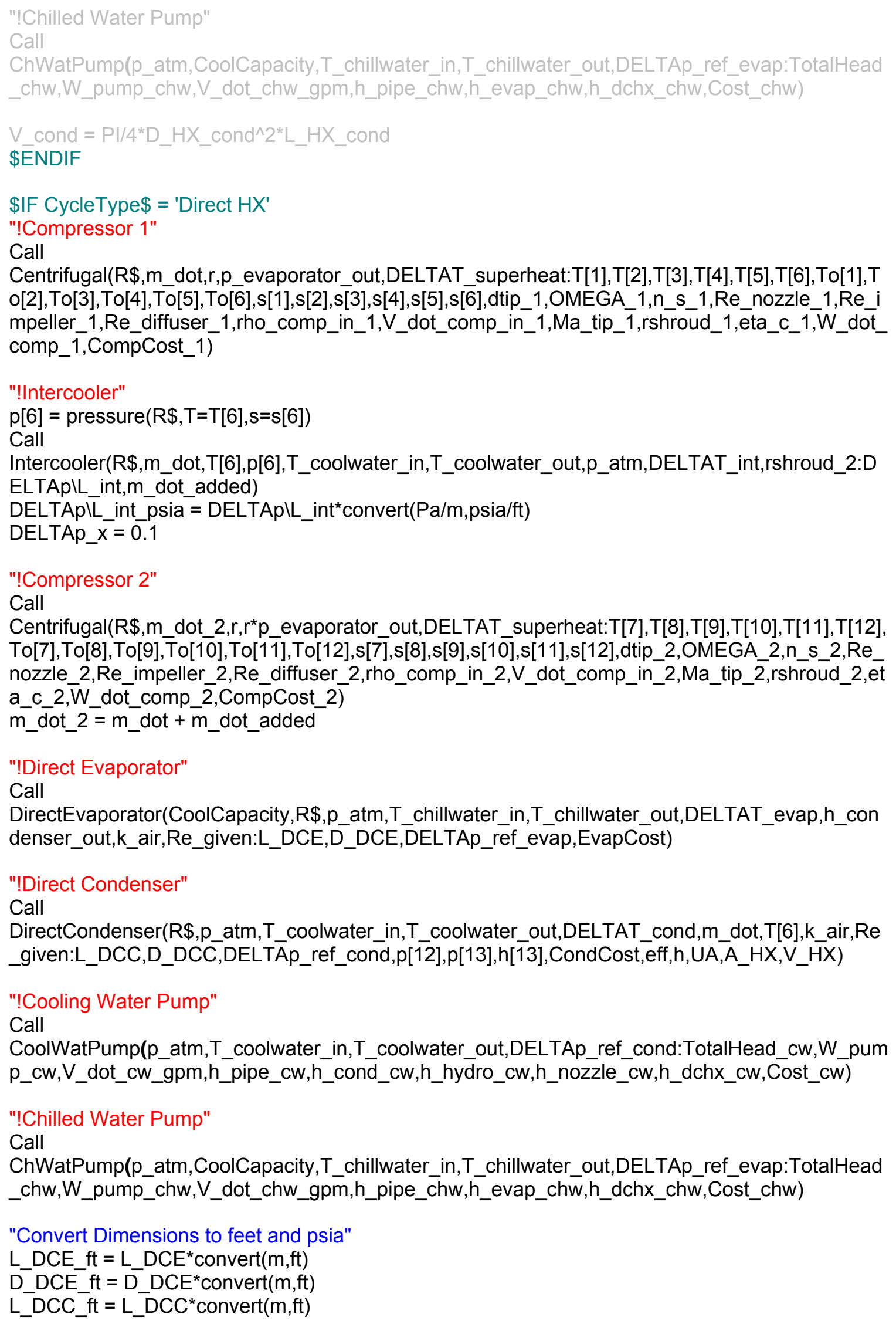




\section{D_DCC_ft $=$ D_DCC ${ }^{*}$ convert $(m, \mathrm{ft})$ \\ $\mathrm{V}$ evap $=\mathrm{PI} / 4^{\bar{*}} \mathrm{D}$ DCE $\mathrm{ft}^{\wedge} 2^{*} \mathrm{~L}$ DCE $\mathrm{ft}$ \\ $V_{-}^{-}$cond $=P I / 4{ }^{*} D_{-} D_{C C}{ }_{-} \mathrm{ft}^{\wedge}{ }^{*} \mathrm{~L}_{-}{ }^{-} \mathrm{DCC}_{-} \mathrm{ft}$ \\ \$ENDIF}

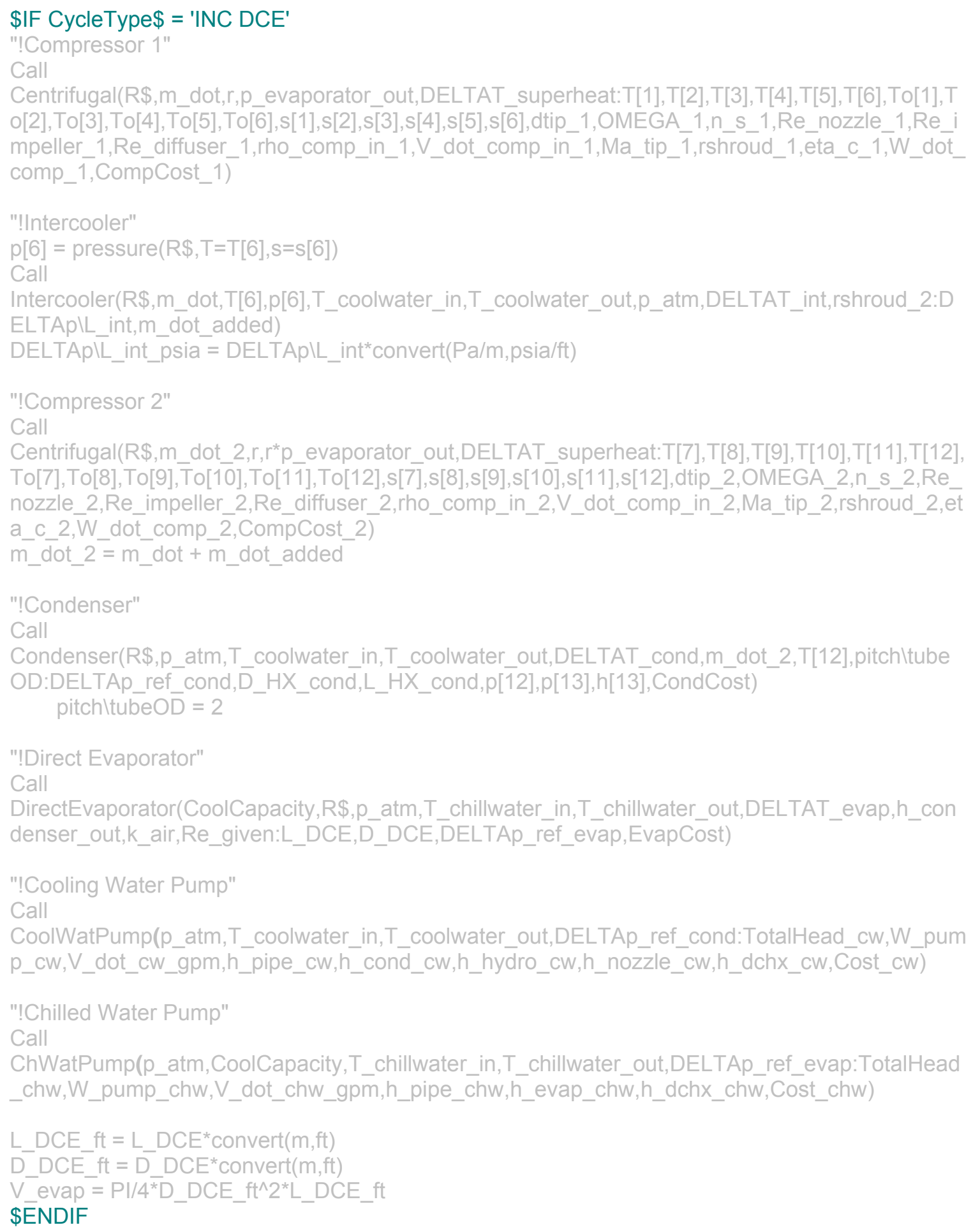




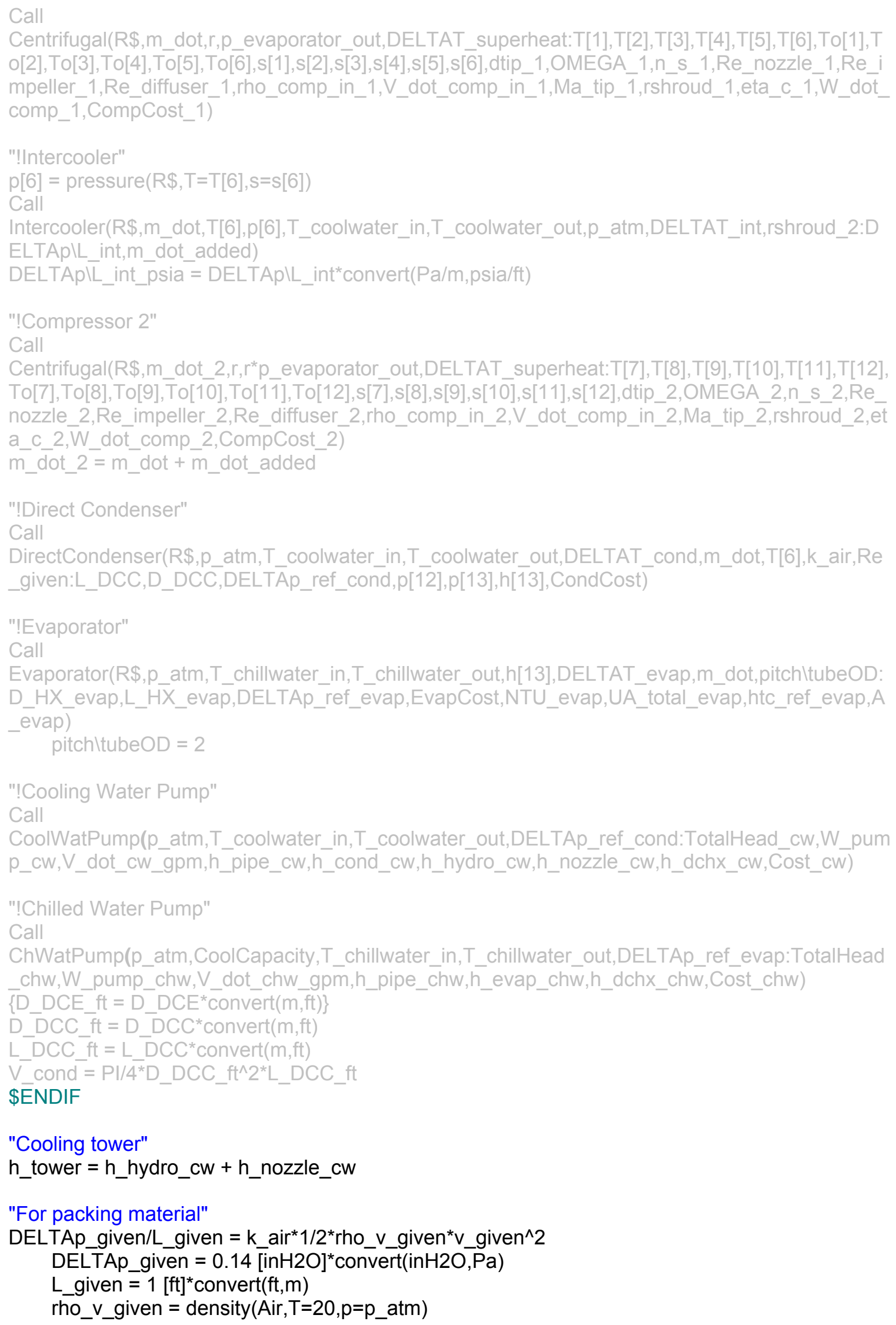




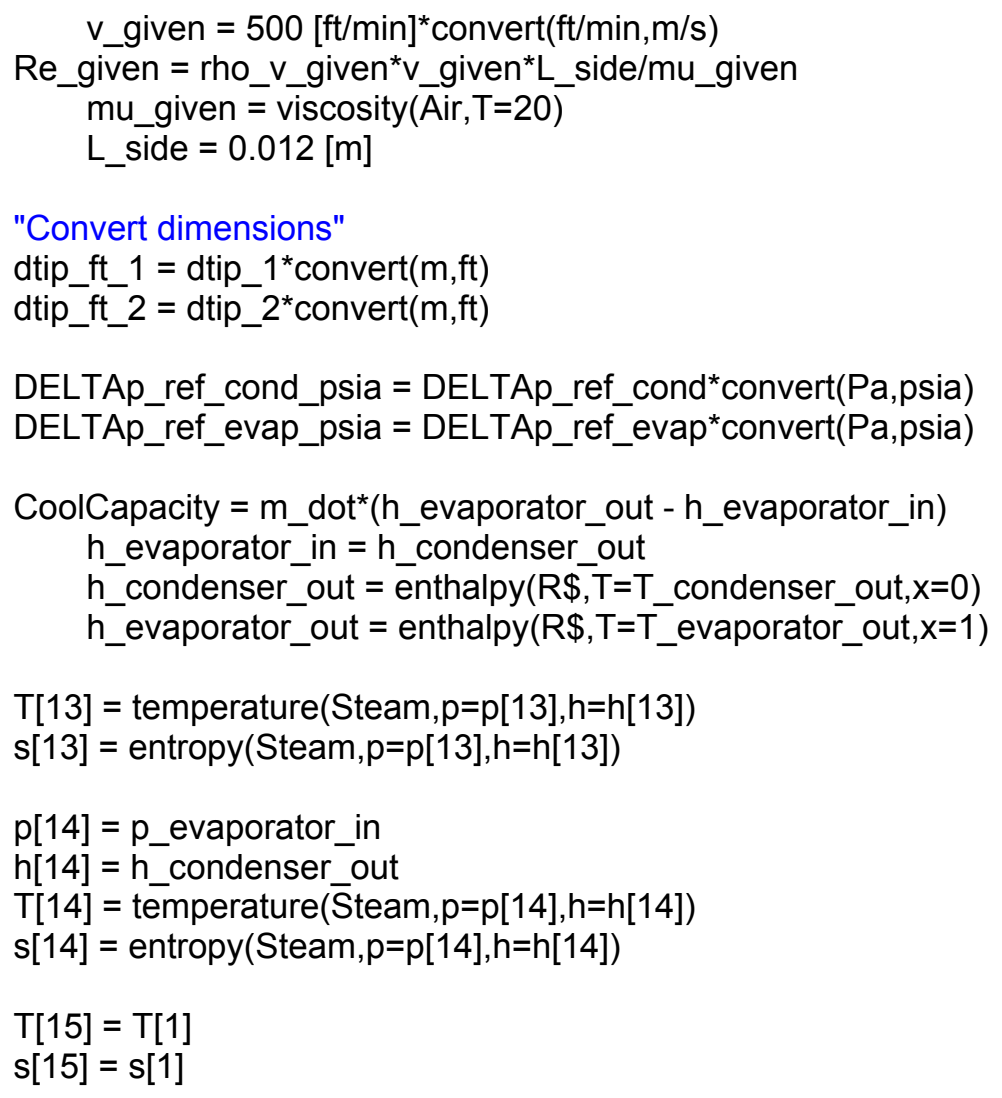

Call CostAnalysis(City\$, EnergyCost, W_dot, CompCost, EvapCost, CondCost, PumpCost,HrsYr,Years: LCC,OC,E) 


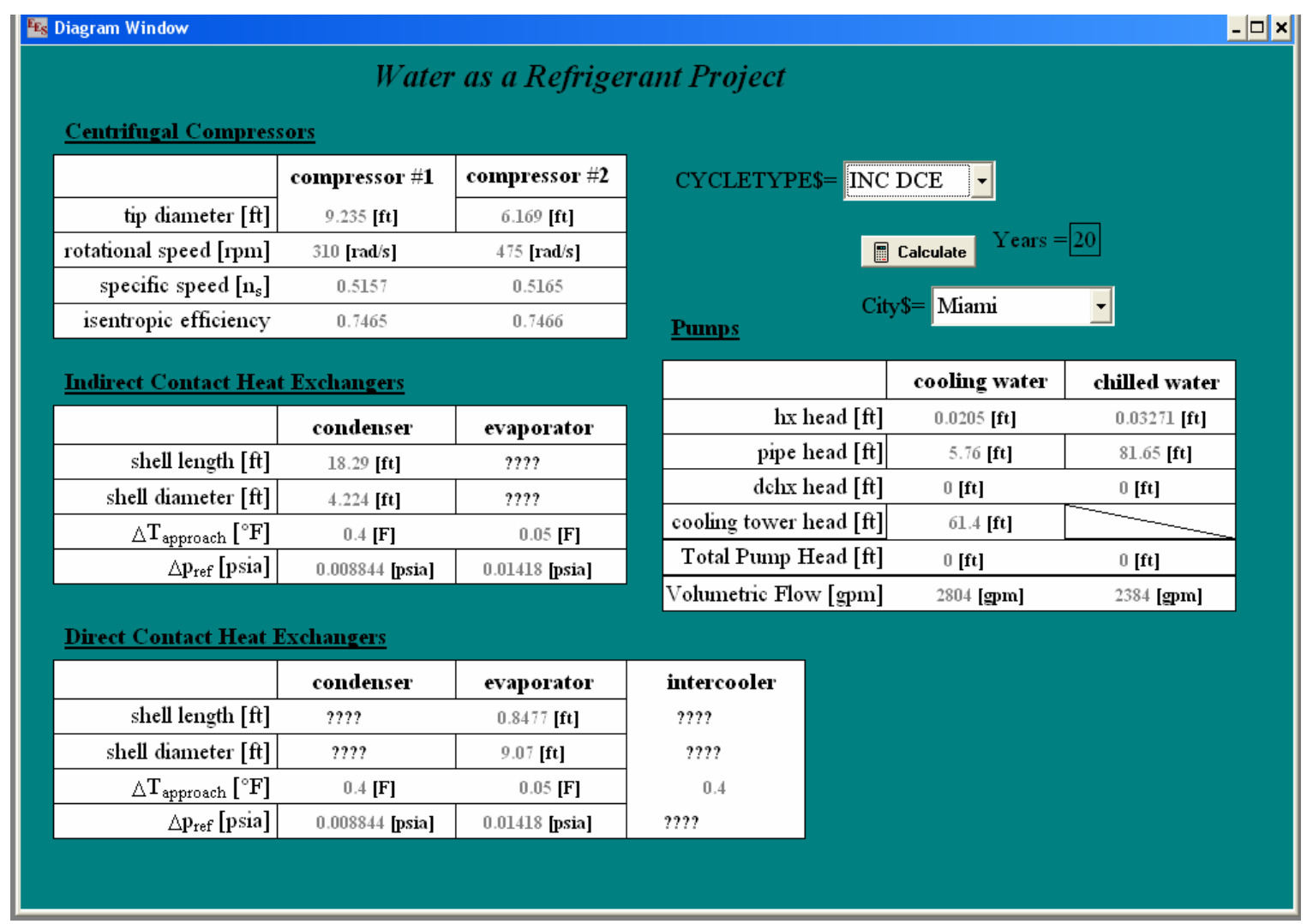

Figure 63 Screen capture of diagram window used for EES program.

\section{EES Code for R134a Cycle}

Module

Centrifugal(R\$,T_evaporator_out,DELTAT_superheat,p[1],p[6],r,m_dot:T[1],T[2],T[3],T[4],T[5],T[6 ],p[2],p[3],p[4],p[5],s[1],s[2],s[3],s[4],s[5],s[6], v[1],v[2],v[3],v[4],v[5],v[6],h[1],h[2],h[3],h[4],h[5],h[6],r tip,n_s,OMEGA,V_dot_cfm,W_dot,Cost)

"Compressor Calculations"

\$Bookmark Compressor settings

sigma $=0.9$ "Slip factor"

eta_N $=0.95$ "Nozzle efficiency"

rhubtortip $=0.2 \quad$ "Hub-to-tip radius ratio"

diffusion $=0.8 "$ Impeller blade diffusion"

eta_TT_imp $=0.9$ "Impeller total-to-total efficiency"

eta_vs $=0.95$ "Vaneless space efficiency"

Ma_4 $=0.8$ "Max allowable Mach number at vaneless space inlet"

C_pr $=0.7 \quad$ "Diffuser coefficient of pressure recovery"

c5lc4 $=0.25$ "Ratio of diffuser inlet and diffuser exit velocities"

$\mathrm{n} \_\mathrm{s}=0.58$

"State (1): Static Inlet Condition"

$\mathrm{T}[1]=\mathrm{T}$ evaporator_out + DELTAT_superheat

$\mathrm{x}[1]=1$

$\mathrm{h}[1]=$ enthalpy $(\mathrm{R} \$, \mathrm{~T}=\mathrm{T}[1], \mathrm{x}=\mathrm{x}[1])$

$c[1]=0$ 
$s[1]=$ entropy $(R \$, T=T[1], x=x[1])$

$v[1]=\operatorname{volume}(R \$, T=T[1], x=x[1])$

"State o(1): Stagnation Inlet Condition"

$\mathrm{ho}[1]=\mathrm{h}[1]+\mathrm{c}[1]^{\wedge} 2 / 2$

$\mathrm{so}[1]=\mathrm{s}[1]$

To[1] =temperature $(\mathrm{R} \$, \mathrm{~h}=\mathrm{ho}[1], \mathrm{s}=\mathrm{so}[1])$

po[1] $=$ pressure $(R \$, h=h o[1], s=s o[1])$

"Stagnation enthalpy rise through compressor"

ho_out_s=enthalpy $\left(R \$, p=r^{*} p[1], s=s[1]\right)$

ho_out=ho[1]+(ho_out_s-ho[1])/eta_c

Deltah_o=ho_out-ho[1]

"Euler's Equation and Slip Factor"

utip=sqrt(Deltah_o/sigma) "[m/s]" "Impeller tip velocity"

$c[2]=u^{*}{ }^{*} 0.35 \overline{ } \overline{ }[\mathrm{m} / \mathrm{s}] " \quad$ "refrigerant impeller inlet velocity"

"Nozzle Relations"

ho[2]=ho[1]

$\mathrm{h}[2]=\mathrm{ho}[2]-\mathrm{c}[2]^{\wedge} 2 / 2$

h2s=ho[2]-(ho[2]-h[2])/eta_N "nozzle efficiency equation"

"State (2): Static Nozzle Condition"

$\mathrm{p}[2]=$ pressure $(\mathrm{R} \$, \mathrm{~h}=\mathrm{h} 2 \mathrm{~s}, \mathrm{~s}=\mathrm{s}[1])$

$\mathrm{s}[2]=$ entropy $(\mathrm{R} \$, P=P[2], \mathrm{h}=\mathrm{h}[2])$

$v[2]=$ volume $(R \$, P=P[2], h=h[2])$

$\mathrm{T}[2]=$ temperature $(\mathrm{R} \$, P=P[2], h=h[2])$

$x[2]=$ quality $(R \$, P=P[2], h=h[2])$

"State o(2): Stagnation Nozzle Condition"

$\mathrm{so}[2]=\mathrm{s}[2]$

To[2]=temperature $(\mathrm{R} \$, \mathrm{~h}=\mathrm{ho}[2], \mathrm{s}=\mathrm{so}[2])$

$\mathrm{Po}[2]=$ pressure $(\mathrm{R} \$, \mathrm{~h}=\mathrm{ho}[2], \mathrm{s}=\mathrm{so}[2])$

"Impeller Geometry"

OMEGA=n_sDeltah_o^0.75/(m_dot* $\left.{ }^{*}[1]\right)^{\wedge} 0.5$ "Impeller rotational velocity"

rtip=utip/omega

dtip $=2^{*}$ rtip

rhub $=$ rhubtortip ${ }^{*}$ rtip

rshroud=sqrt(rhub^2+m_dot $\left.{ }^{\star} v[2] /\left(c[2]^{\star} p i\right)\right)$

"Geometric constraint of hub-to-tip ratio"

"Mass conservation"

"Blade entrance velocities"

u_hub = omega*rhub

"kinematic relationship between rotational speed

and impeller speed"

u_shroud $=$ omega ${ }^{*}$ rshroud

"Calculate the hub and shroud blade angles"

Beta_hub $=\arctan \left(\right.$ u_hub/c[2]) ${ }^{*}$ convert(rad,deg)

"from velocity vector diagrams"

Beta_shroud $=\arctan \left(u \_s h r o u d / c[2]\right)^{*} \operatorname{convert}($ rad, deg $)$

"Determine the relative velocity at the inlet shroud and the relative Mach number at the inlet shroud"

w_shroud $=\operatorname{sqrt}\left(\right.$ u_shroud $\left.2+c[2]^{\wedge} 2\right)$

"Impeller Blade diffusion" 


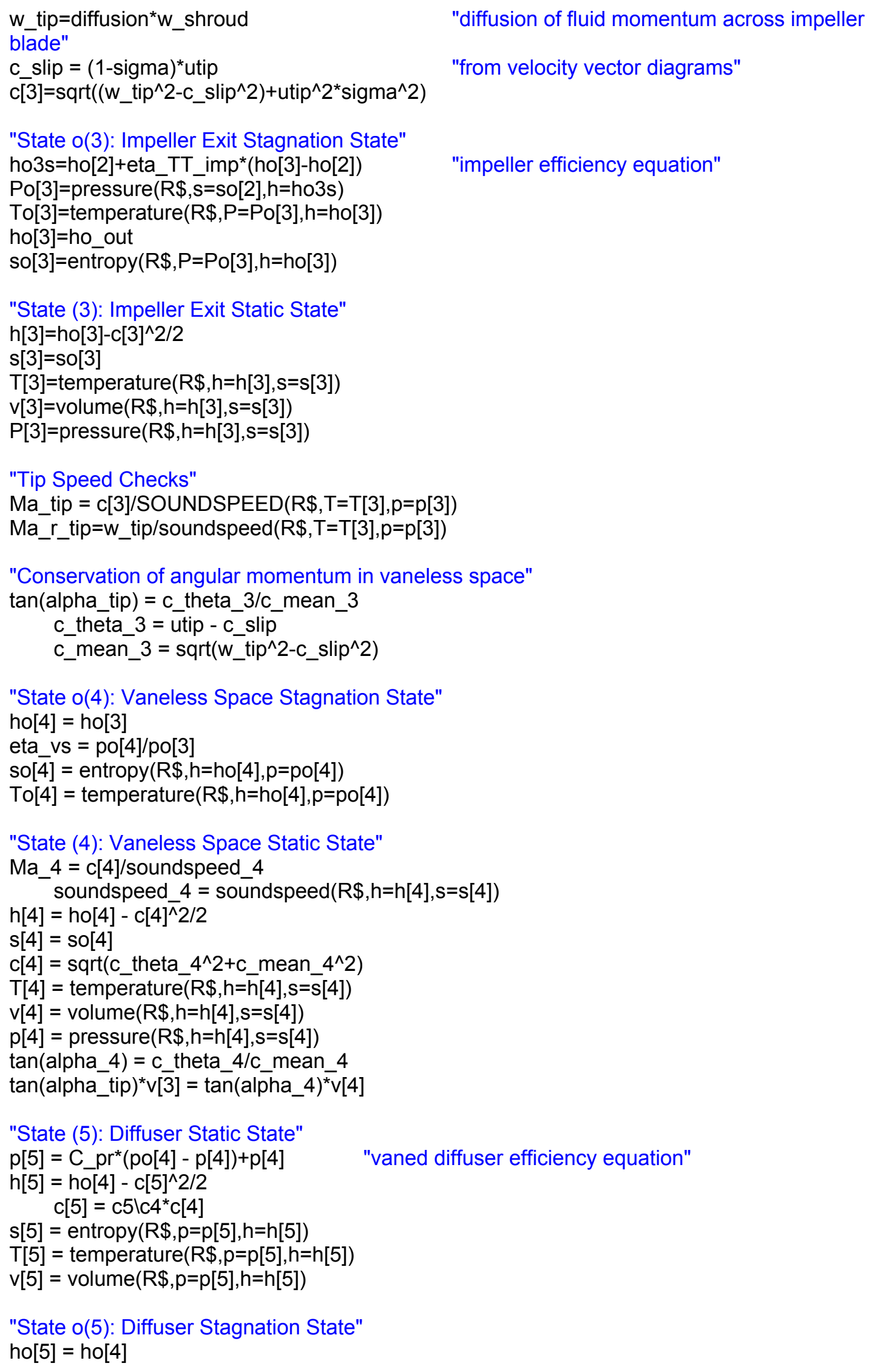

"State o(5): Diffuser Stagnation State" ho[5] = ho[4] 


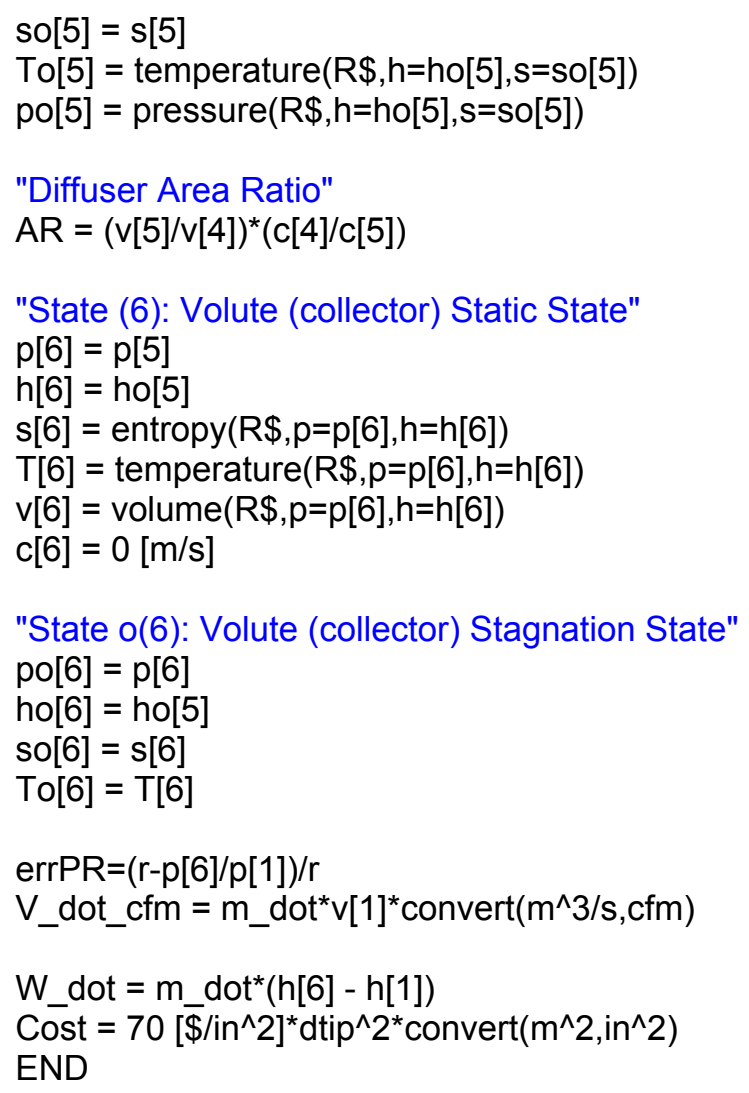

"State 2: Refrigerant out"

$\mathrm{x}[2]=0$

$\mathrm{T}[2]=\mathrm{T}$ _coolwater_out + DELTAT_cond "Condenser approach temperature"

$\mathrm{p}[2]=$ pressure $(\mathrm{R} \$, \mathrm{~T}=\mathrm{T}[2], \mathrm{x}=\mathrm{x}[2])$

$\mathrm{h}[2]=$ enthalpy $(\mathrm{R} \$, \mathrm{~T}=\mathrm{T}[2], \mathrm{x}=\mathrm{x}[2])$

$\mathrm{s}[2]=$ entropy $(\mathrm{R} \$, \mathrm{~T}=\mathrm{T}[2], \mathrm{x}=\mathrm{x}[2])$

$v[2]=$ volume $(R \$, T=T[2], x=x[2])$

"State 3: Cooling water in"

$\mathrm{T}[3]=\mathrm{T}$ _coolwater_in

$\mathrm{p}[3]=\left(1+\mathrm{p} \_ \text {drop_cw/100 }[\mathrm{Pa}]\right)^{*} \mathrm{p}[4]$

$\mathrm{h}[3]=$ enthalpy $($ Water, $\mathrm{T}=\mathrm{T}[3], \mathrm{p}=\mathrm{p}[3])$

"condenser water inlet temperature"

$\mathrm{s}[3]=$ entropy $($ Water, $\mathrm{T}=\mathrm{T}[3], \mathrm{p}=\mathrm{p}[3])$

"condenser water pressure drop"

$\mathrm{v}[3]=$ volume $($ Water $, T=T[3], p=p[3])$ 
"State 4: Cooling water out"

$\mathrm{T}[4]=\mathrm{T}$ _coolwater_out

"condenser water exit temperature"

$\mathrm{p}[4]=\mathrm{p}$ atm

$\mathrm{h}[4]=$ enthalpy $($ Water, $\mathrm{T}=\mathrm{T}[4], \mathrm{p}=\mathrm{p}[4])$

$\mathrm{s}[4]=$ entropy $($ Water, $\mathrm{T}=\mathrm{T}[4], \mathrm{p}=\mathrm{p}[4])$

$v[4]=$ volume $($ Water $, T=T[4], p=p[4])$

"Average cooling water side properties"

T_cW = average $(\mathrm{T}[3], \mathrm{T}[4])$

p_cw $=$ average $(p[3], p[4])$

"bulk temperature"

"bulk pressure"

$c P \_c w=c P\left(\right.$ Water, $\left.T=T \_c w, p=p \_c w\right) \quad$ "constant pressure specific heat"

rho_cw $=$ density $\left(\right.$ Water, $\left.T=T+c w, p=p \_c w\right)$ "density"

mu_cw $=$ viscosity $\left(\right.$ Water $\left., T=\bar{T} \_c w, p=\bar{p}_{-} c w\right)$ "viscosity"

$\mathrm{k} \_\mathrm{cW}=$ conductivity $\left(\mathrm{Water}, \mathrm{T}=\overline{\mathrm{T}} \_\mathrm{cW}, \mathrm{p}=\overline{\mathrm{p}} \mathrm{c} \mathrm{cW}\right) \quad$ "thermal conductivity"

Pr_cw $=$ prandtl $\left(\right.$ Water, $\left.T=T \_c w, p=p_{-} c w\right)$ "Prandtl number"

vol_cw $=$ volume $\left(\right.$ Water, $\left.T=\bar{T} \_c w, p=\bar{p} \_c w\right)$ "specific volume"

"Heat exchanger geometric relationships"

d_tube_i_inch $=0.7$

"tube inner diameter"

d_tube_i $=$ d_tube_i_inch*onvert(in, $m$ )

$\mathrm{t}$ tube $=0.025[\mathrm{in}]^{*}$ convert(in, $\mathrm{m}$ )

D_HX = D_HX_inch ${ }^{*}$ convert(in, $\left.m\right)$

$\mathrm{L}_{-} \mathrm{HX}=\mathrm{L} \_\mathrm{HX}$ inch*convert(in, $\mathrm{m}$ )

D_HX_ft $=$ D_HX_inch*convert(in,ft)

$\mathrm{L}_{-} \mathrm{HX} \mathrm{ft}_{-}=\mathrm{L} \_\mathrm{HX}$ inch ${ }^{*}$ convert(in,ft)

N_baffle $=10$

"tube thickness"

"shell diameter"

"shell length"

"number of baffles"

"Tube properties"

k_tube = k_(T\$,CONVERTTEMP $\left.\left(C, K, T \_c w\right)\right)$ "tube thermal conductivity"

"Refrigerant side properties"

rho_I_ref $=\operatorname{density}(\mathrm{R} \$, \mathrm{~T}=\mathrm{T}[2], \mathrm{x}=0) \quad$ "liquid refrigerant density"

rho_v_ref $=\operatorname{density}(\mathrm{R} \$, \mathrm{~T}=\mathrm{T}[2], \mathrm{x}=1) \quad$ "vapor refrigerant density"

k_I_ref = conductivity $(R \$, T=T[2], x=0) \quad$ "liquid refrigerant thermal conductivity"

hfg_ref = enthalpy $(R \$, T=T[2], x=1)-h[2] \quad$ "enthalpy of vaporization"

mu_I_ref $=$ viscosity $(R \$, T=T[2], x=0) \quad$ "liquid refrigerant viscosity"

mu_v_ref $=$ viscosity $(R \$, T=T[2], x=1) \quad$ "vapor refrigerant viscosity"

"Energy balance"

Q_dot_cond $=m$ _dot_ref* $(\mathrm{h}[1]-\mathrm{h}[2]) \quad$ "refrigerant side energy balance"

Q_dot_cond $=m_{-}$dot_cw* $(\mathrm{h}[4]-\mathrm{h}[3]) \quad$ "condenser water side energy balance"

Q_dot_cond_ton $=\mathrm{Q}_{-}$dot_cond ${ }^{*}$ convert $(\mathrm{W}$,tons $)$

"Epsilon-NTU relationships"

eff_cond $=(T[4]-T[3]) /(T[2]-T[3])$

NTU $=$-In(1-eff_cond)

UA_req $=N T U^{\bar{*}} \mathrm{~m} \_$dot $\_\mathrm{CW}^{*} \mathrm{CP} \_\mathrm{cW}$

"NTU relationship for capacitance ratio of zero"

"definition of conductance"

"Tube geometry"

d_tube_o $=$ d_tube_i $+2^{*} t$ tube

pitch $=$ pitchltubeO $\bar{D}^{*}$ d_tube_o

"tube outer diameter"

"spacing between tubes"

A $H X=p i^{*} D H X^{\wedge} 2 / 4$

"heat exchanger shell cross-sectional area"

N_tubes_Xsection $=$ A_HX/ $\left(2^{*} \mathrm{pitch}^{\wedge} 2^{*} \sin (\mathrm{pi} / 3)^{*} \cos (\mathrm{pi} / 3)\right)$ "number of tubes in the shell crosssection" 


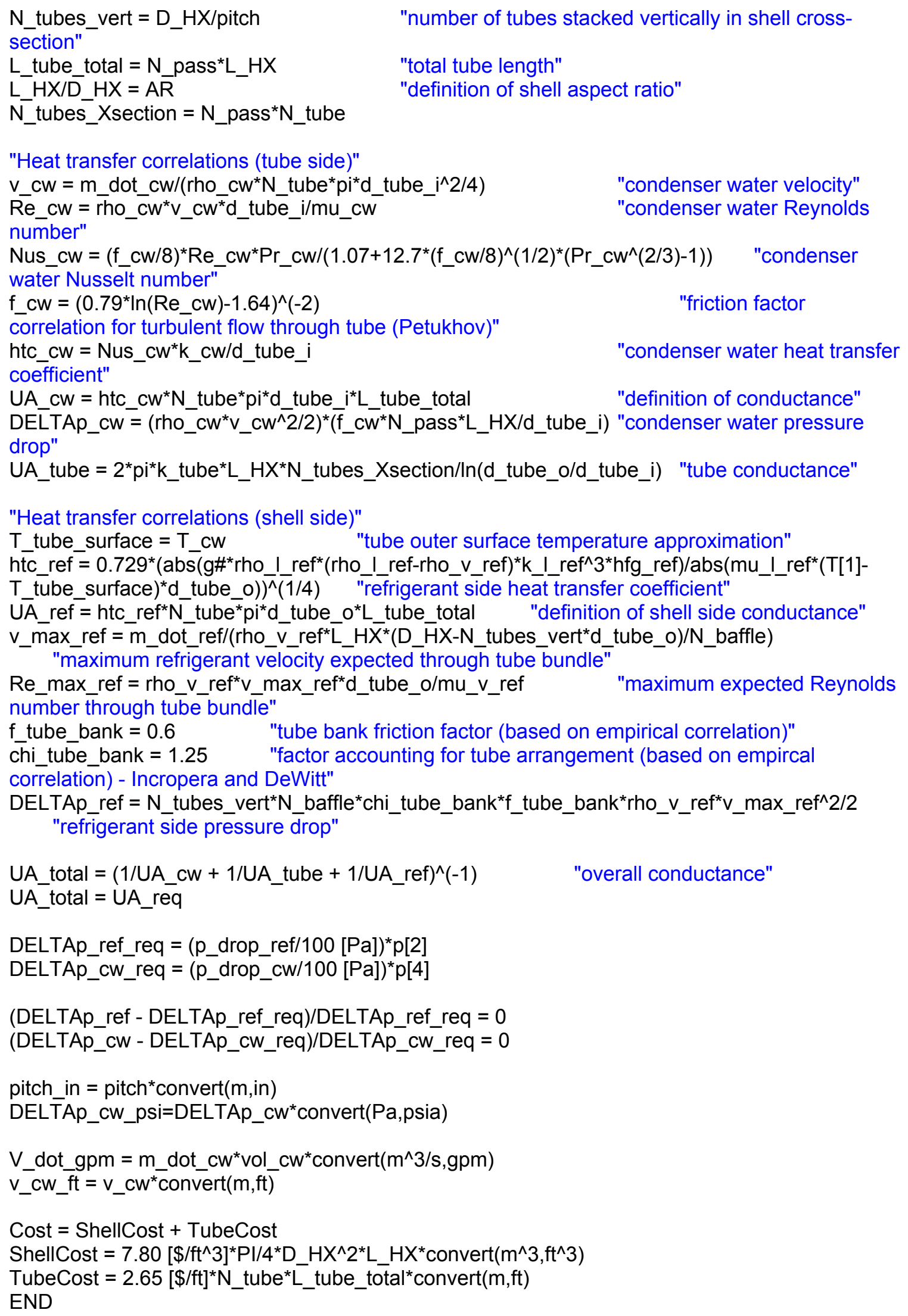




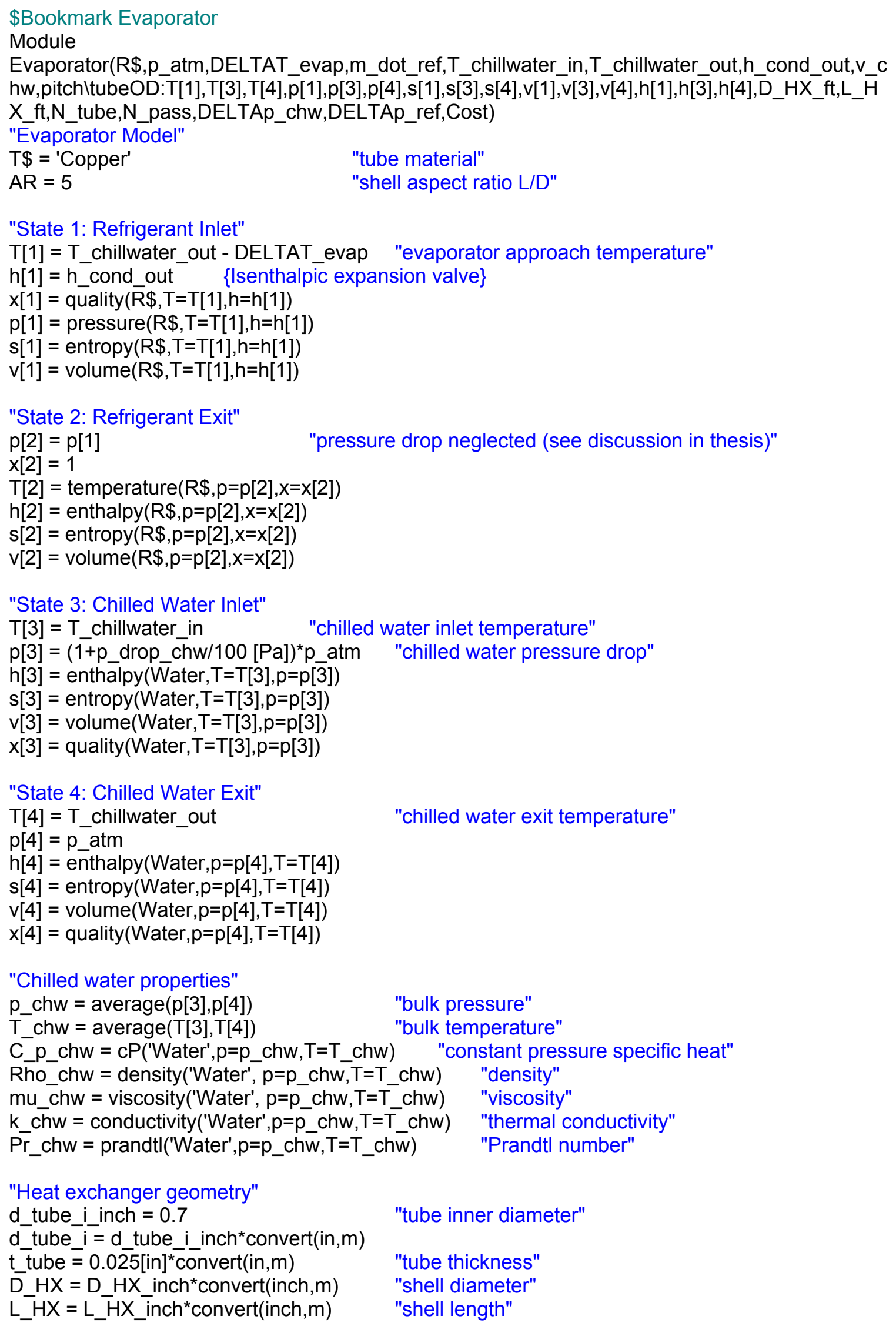


D_HX_ft $=$ D_HX_inch ${ }^{*}$ convert(inch,ft)

$\mathrm{L} \_\mathrm{HX} \_\mathrm{ft}=\mathrm{L} \_\mathrm{HX}$ inch ${ }^{*}$ convert(inch,ft)

N_baffle $=1 \overline{0}$

"number of baffles"

"Tube Properties"

k_tube $=$ k_(T\$,converttemp $(\mathrm{C}, \mathrm{K},(\mathrm{T}[3]+\mathrm{T}[4]) / 2))$ "tube thermal conductivity"

"Refrigerant properties"

rho _ ref $=\operatorname{density}(\mathrm{R} \$, \mathrm{~T}=\mathrm{T}[2], \mathrm{x}=0)$

rho_v_ref $=\operatorname{density}(\mathrm{R} \$, \mathrm{~T}=\mathrm{T}[2], \mathrm{x}=1)$

k_I_ref $=$ conductivity $(R \$, T=T[2], x=0)$

"liquid refrigerant density"

"vapor refrigerant density"

k_v_ref = conductivity $(R \$, T=T[2], x=1)$

"liquid refrigerant thermal conductivity"

hfg_ref $=h[2]-$ enthalpy $(R \$, T=T[2], x=0)$

"vapor refrigerant thermal conductivity"

mu_I_ref $=$ viscosity $(R \$, T=T[2], x=0)$

"enthalpy of vaporization"

mu_v_ref $=$ viscosity $(R \$, T=T[2], x=1)$

"liquid refrigerant viscosity"

"vapor refrigerant viscosity"

$c P \_v=C P(R \$, T=T[2], x=1)$

"liquid refrigerant constant pressure specific heat"

nu_v_ref $=$ mu_v_ref/rho_v_ref

Pr_ref $=$ prandt $(\bar{R} \$, T=T[\overline{2}], \bar{x}=1)$

"vapor refrigerant kinematic viscosity"

"vapor refrigerant Prandtl number"

"Energy balance"

Q_dot_evap $=$ m_dot_ref $f^{*}(\mathrm{~h}[2]-\mathrm{h}[1]) \quad$ "refrigerant side energy balance"

Q_dot_evap $=m_{-}$dot_chw* $(\mathrm{h}[3]-\mathrm{h}[4])$

Q_dot_evap_ton $=$ Q_dot_evap ${ }^{*}$ convert $(W$,tons $)$

"Epsilon-NTU relations"

eff_evap $=(T[3]-T[4]) /(T[3]-T[1])$

NTU $=-\ln (1$-eff_evap)

UA_req $=$ NTU ${ }^{\bar{*}} \mathrm{~m} \_$dot $\_c h w^{*} \mathrm{C} \_p \_c h w$

"NTU relationship for capacitance ratio of zero"

"definition of conductance"

"Tube geometry"

d_tube_o = d_tube_i $+2^{*}$ t_tube

pitch $=\overline{\text { pitchltubeO }} \bar{D}^{*}$ d_tube_o

"tube outer diameter"

"spacing between tubes"

A $H X=p i{ }^{*} D H X^{\wedge} 2 / 4$

"heat exchanger cross-sectional area"

N_tubes_Xsection $=$ A_HX/(2* pitch $\left.^{\wedge} 2^{*} \sin (\mathrm{pi} / 3)^{*} \cos (\mathrm{pi} / 3)\right) \quad$ "number of tubes in the shell cross-section"

N_tube_vert $=$ D_HX/pitch

section"

L_tube_total $=$ N_pass* ${ }^{*} \_H X$

L_HX/D_HX $=A \bar{R}$

"chilled water energy balance"

N_tubes_Xsection $=\mathrm{N} \_$pass ${ }^{*} \mathrm{~N} \_$tube

"Heat transfer coefficient calculations for tube side"

v_chw $=$ m_dot_chw/(rho_chw ${ }^{*} N \_t u b e^{*} \mathrm{pi}^{*} \mathrm{~d} \_$tube_i^2/4) "chilled water velocity"

Re_chw $=$ rho chw $^{*} v$ chw $^{*}$ d tube $\mathrm{i} / \mathrm{mu}$ chw

"number of tubes stacked vertically in shell cross-

"total tube length"

"definition of aspect ratio"

$f \_c \bar{h} w=\left(0.79^{\star} \ln \left(\operatorname{Re} \_ \text {chw }\right)-1 . \overline{6} 4\right)^{\wedge}(-\overline{2})$ "Petukhov correlation"

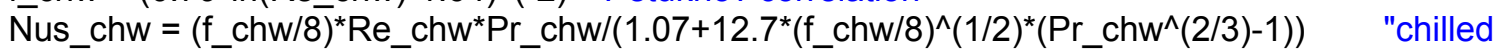
water Nusselt number"

htc_chw $=$ Nus_chw*k_chw/d_tube_i

"chilled water heat transfer

coefficient"

UA_chw $=h t c \_c h w^{*} N \_t u b e s \_X s e c t i o n{ }^{*} \mathrm{pi}^{*} \mathrm{~d} \_$tube_i*L_HX "definition of conductance"

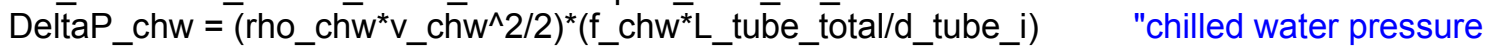
drop"

"Tube conductance"

UA_tube $=2{ }^{*} \mathrm{pi}^{*} \mathrm{k} \_t u b{ }^{*} \mathrm{~L} \_H X^{*} \mathrm{~N} \_t$ tubes_Xsection/In(d_tube_o/d_tube_i) "tube conductance" 
"Heat transfer coefficient calculations for shell side"

DELTAT_e = T_tube_surface - T[1]

"tube surface superheat"

$\mathrm{C}=0.62$ "for horizontal cylinders"

hfg_prime $=$ hfg_ref $+0.80^{*} \mathrm{CP} \_\mathrm{v}^{*} \mathrm{DELTAT}$ e $\quad$ "modified enthalpy of vaporization"

Nus_D_bar $=\mathrm{C}^{*}\left(\mathrm{~g} \#^{*}(\right.$ rho_I_ref -

rho_v_ref)*hfg_prime*d_tube_o^3/(nu_v_ref*k_v_ref* DELTAT_e $))^{\wedge}(1 / 4)$ "heat transfer coefficient correlation for condensation over a tube bundle"

T_tube_surface $=(T[3]+T[4]) / 2$

"tube surface temperature"

htc_ref $=$ Nus_D_bar*k_I_ref/d_tube_o

"refrigerant side heat transfer

coefficient"

UA_ref $=$ htc_ref ${ }^{*} N \_p a s s^{*} N \_t u b e^{*} p^{*}{ }^{*} d$ tube_o* ${ }^{*} \_H X \quad$ "definition of conductance"

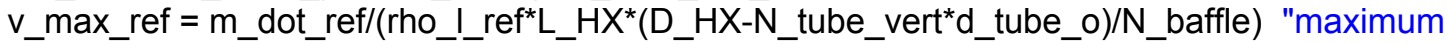
expected refrigerant velocity through tube bundle"

Re_max_ref $=$ rho_I_ref*v_max_ref*d_tube_o/mu_I_ref "maximum expected Reynolds number"

f_tube_bank $=0.6 \quad$ "tube bank friction factor (based on empirical correlation)"

chi_tube_bank $=1.25 \quad$ "factor accounting for tube arrangement (based on empirical

correlation) - Incropera and DeWitt"

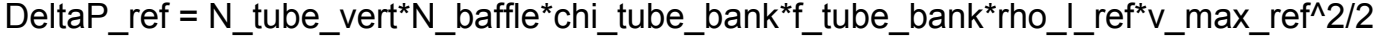

"refrigerant side pressure drop"

UA_total $=(1 / \text { UA_chw }+1 / \text { UA_tube }+1 / \text { UA_ref })^{\wedge}(-1) \quad$ "overall conductance"

errUA $=(U A$ _total-UA_req $) / U A \_r e q$

errUA $=0$

errDeltaP_chw $=($ DeltaP_chw - DeltaP_chw_req $) / D e l t a P \_c h w \_r e q$

errDeltaP_chw $=0$

DELTAp_chw_req $=\left(p \_d r o p \_c h w / 100[P a]\right)^{*} p[4]$

DELTAp_ref $=(\text { p_drop_ref } / 100[P a])^{\star} p[1]$

pitch_in $=$ pitch ${ }^{*} \operatorname{convert}(\mathrm{m}, \mathrm{in})$

DELTAp_chw_psi $=$ DELTAp_chw*convert(Pa,psia)

chw_head_ft=DELTAp_chw/(rho_chw* $\left.{ }^{*} \#\right)^{*} \operatorname{convert}(\mathrm{m}, \mathrm{ft})$

Cost $=$ ShellCost + TubeCost

ShellCost $=7.80\left[\$ / \mathrm{ft}^{\wedge} 3\right]^{*} \mathrm{PI} / 4^{*} \mathrm{D} \_H X^{\wedge} 2^{*} \mathrm{~L} \_H X^{*}$ convert $\left(\mathrm{m}^{\wedge} 3, \mathrm{ft}^{\wedge} 3\right)$

TubeCost $=2.65[\$ / \mathrm{ft}]^{*} \mathrm{~N} \_$tube ${ }^{*} \mathrm{~L} \_$tube_total ${ }^{*} \operatorname{convert}(\mathrm{m}, \mathrm{ft})$

END

\$Bookmark Chilled Water Pump

Subprogram

ChWatPump(p_atm,CoolCapacity,T_chillwater_in,T_chillwater_out,p_drop_evap:TotalHead,W_p ump,V_dot_gpm,W_dot,Cost)

AreaPerTon $=400\left[\bar{f}^{\wedge} \wedge /\right.$ tons $] \quad$ "Square footage of cooled area per ton of capacity"

$\mathrm{N}$ floor $=4 \quad$ "Number of floors"

T_chw $=$ average $(T$ chillwater_in, $T$ _chillwater_out $)$

rho_chw $=\operatorname{density}\left(\bar{W} \text { ater, } T=T_{-} \text {chw, } p=\left(p \_a t m+p \_p u m p \_P a / 2\right)\right)^{*} \operatorname{convert}\left(\mathrm{kg} / \mathrm{m}^{\wedge} 3, \mathrm{lbm} / \mathrm{ft}^{\wedge} 3\right)$

g_c $=32.2\left[\mathrm{lbm}-\mathrm{ft} / \mathrm{lbf}-\mathrm{s}^{\wedge} 2\right] \quad$ "gravitational constant"

eta_pump $=0.8042 \quad$ "!Depends on pump model selected"

h_evap $=$ p_drop_evap/(rho_chw* ${ }^{*}$ convert $\left.\left(\mathrm{lbm} / \mathrm{ft}^{\wedge} 3, \mathrm{~kg} / \mathrm{m}^{\wedge} 3\right)^{*} \mathrm{~g} \#\right)^{*} \operatorname{convert}(\mathrm{m}, \mathrm{ft})$

A_total $=$ AreaPerTon ${ }^{*}$ CoolCapacity ${ }^{*}$ convert(W,tons) $\quad$ "Total square footage"

A_building = A_total/N_floor "Building footprint" 


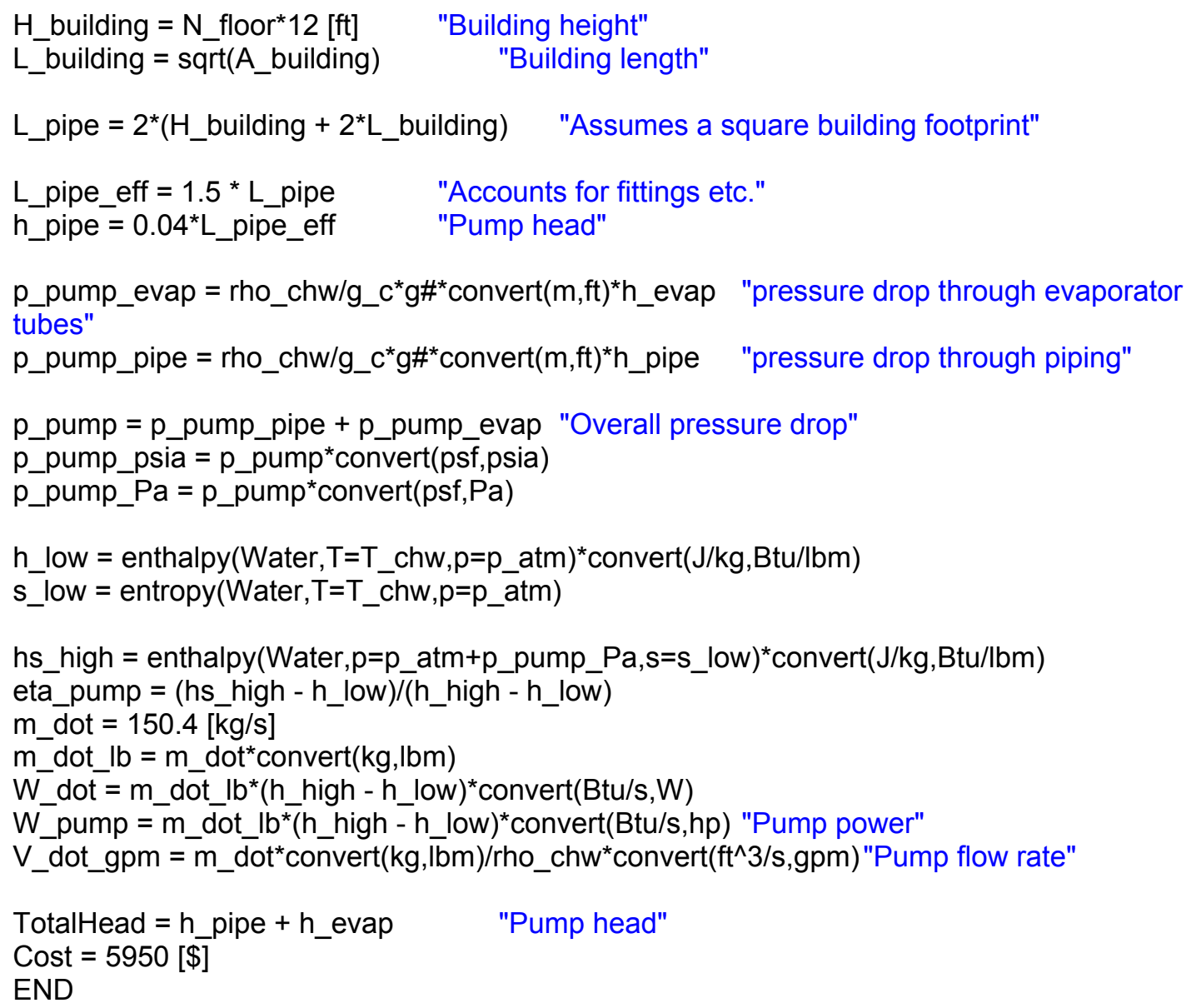




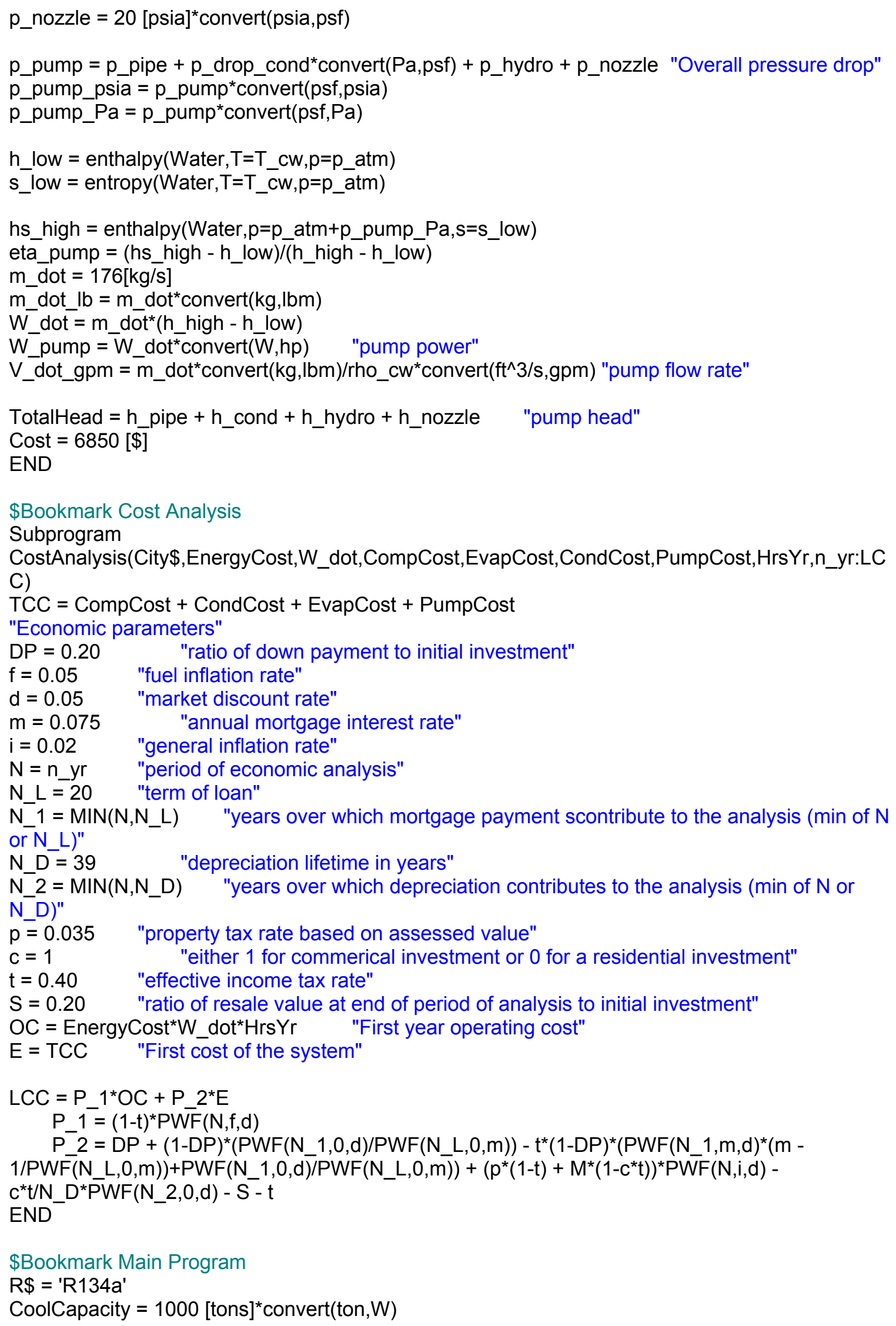


\{ARI Conditions\}

T_chillwater_in = CONVERTTEMP $(F, C, 54) \quad$ "chilled water inlet temperature"

T_chillwater_out $=$ CONVERTTEMP $(F, C, 44) \quad$ "chilled water exit temperature"

T_coolwater_in = CONVERTTEMP $(\mathrm{F}, \mathrm{C}, 85) \quad$ "condenser water inlet temperature"

T_coolwater_out $=$ CONVERTTEMP $(F, C, 95) \quad$ "condenser water exit temperature"

DELTAT_cond $=$ DELTAT_cond_F*convert $(F, C)$

DELTAT_cond_F $=5[\mathrm{~F}]$ "Condenser approach temperature"

DELTAT_evap $=$ DELTAT_evap_F*convert $(F, C)$

DELTAT_evap_F $=3.5[\mathrm{~F}]$ "Evaporator approach temperature"

DELTAT_superheat $=0[\mathrm{C}]$

"Set up guess values (help EES iteration loop - these equations have no bearing on solution other than that it helps EES converge)"

$\mathrm{Tg}=16[\mathrm{C}]$

$\mathrm{Pg}=$ pressure $(\mathrm{R} \$, \mathrm{~T}=\mathrm{Tg}, \mathrm{x}=1)^{*} 0.8$

$v g=$ volume $(R \$, T=T g, P=P g)$

$\mathrm{sg}=$ entropy $(\mathrm{R} \$, \mathrm{~T}=\mathrm{Tg}, \mathrm{P}=\mathrm{Pg})$

hg=enthalpy $(\mathrm{R} \$, \mathrm{~T}=\mathrm{Tg}, \mathrm{P}=\mathrm{Pg})$

soundspeedg $=$ soundspeed $(R \$, T=T g, p=P g)$

p_atm = 101325 "[Pa]" $\quad$ AAtmospheric pressure

T_condenser_out $=T$ _coolwater_out + DELTAT_cond

T_evaporator_out $=T_{-}$_chillwater_out - DELTAT_evap

$\mathrm{p} \_$condenser_out $=$pressure $(\mathrm{R} \$, \mathrm{x}=0, \mathrm{~T}=\mathrm{T}$ _condenser_out $)$

p_condenser_in $=p \_c o n d e n s e r \_o u t+D E L T A p \_c o n d$

p_evaporator_out $=$ pressure $(R \overline{\$}, x=1, T=T$ evaporator_out $)$

p_evaporator_in = p_evaporator_out + DELTAp_ref_evap

CoolCapacity $=m_{-} \operatorname{dot}^{*}(\mathrm{~h}[1]-\mathrm{h}[9])$

"Specify ALL pressures"

$r=p \_c o n d e n s e r$ in/p_evaporator_out "Compressor Pressure Ratio"

$\mathrm{p}[1]=\mathrm{p} \_$evaporator_out

$\mathrm{p}[6]=\mathrm{r}^{\star} \mathrm{p}[1]$

$\mathrm{p}[7]=\mathrm{p}[6]-\mathrm{h}$ _fraction* $\mathrm{p}_{-} d \mathrm{drop} \_\mathrm{cw}$

$h \_$fraction $=(h[7]-\bar{h}[8]) /(\bar{h}[6]-h[8])$

$\mathrm{h}[7]=$ enthalpy $(R \$, p=p[7], x=x[7])$

$$
x[7]=1
$$

$\mathrm{T}[7]=$ temperature $(\mathrm{R} \$, \mathrm{p}=\mathrm{p}[7], \mathrm{x}=\mathrm{x}[7])$

$s[7]=\operatorname{entropy}(R \$, p=p[7], x=x[7])$

$v[7]=$ volume $(R \$, p=p[7], x=x[7])$

d_tip_in $=2^{*}$ rtip ${ }^{*}$ convert $(\mathrm{m}$,in)

OMEGA_rpm $=$ OMEGA ${ }^{*}$ convert(rad/s,rpm)

CompressorPower $=m_{-} \operatorname{dot}^{*}(h[6]-h[1])^{*} \operatorname{convert}(\mathrm{W}, \mathrm{hp})$

v_cw $=$ v_cw_ft*convert $(\mathrm{ft} / \mathrm{s}, \mathrm{m} / \mathrm{s})$ "!"

$v_{-}$chw $=\overline{8}[\mathrm{ft} / \mathrm{s}]^{*}$ convert(ft/s,m/s) "!"

$v_{-} \mathrm{CW}_{-} \mathrm{ft}=8[\mathrm{ft} / \mathrm{s}]$

"Subprogram calls"

Call

Centrifugal(R\$,T_evaporator_out,DELTAT_superheat,p[1],p[6],r,m_dot:T[1],T[2],T[3],T[4],T[5],T[6 ],p[2],p[3],p[4],p[5],s[1],s[2],s[3],s[4],s[5],s[6],v[1],v[2],v[3],v[4],v[5],v[6],h[1],h[2],h[3],h[4],h[5],h[6],r tip,n_s,OMEGA,V_dot_comp,W_dot_comp,CompCost) 
pitchltubeOD = 1.5

Call

Condenser(R\$,m_dot,T[6],p[6],T_coolwater_in,T_coolwater_out,DELTAT_cond,p_atm,v_cw,pitch ItubeOD:T[8],T[10],T[11],p[8],p[10],p[11],s[8],s[10],s[11],h[8],h[10],h[11],v[8],v[10],v[11],D_HX_co nd,L_HX_cond,N_tubes_cond,N_pass_cond,p_drop_cw,Nus,DELTAp_cond,CondCost)

Call

Evaporator(R\$,p_atm,DELTAT_evap,m_dot,T_chillwater_in,T_chillwater_out,h[8],v_chw,pitchltub eOD:T[9],T[12],T[13],p[9],p[12],p[13],s[9],s[12],s[13],v[9], v[12],v[13],h[9], h[12],h[13],D_HX_evap,L HX_evap,N_tubes_evap,N_pass_evap,p_drop_chw,DELTAp_ref_evap,EvapCost)

DELTAp_ref_evap_psi $=$ DELTAp_ref_evap*convert(Pa,psia)

Call

ChWatPump(p_atm,CoolCapacity,T_chillwater_in,T_chillwater_out,p_drop_chw:TotalChilledWate rHead,P_pump_chw,V_dot_gpm_chw,W_dot_pump_chw,PumpCost_chw)

Call

CoolWatPump(p_atm,T_coolwater_in,T_coolwater_out,p_drop_cw:TotalCoolingWaterHead,P_pu mp_cw,V_dot_gpm_cw,W_dot_pump_cw,PumpCost_cw)

p_1_psia $=p[1]^{*}$ convert $(P a, p s i a)$

p_drop_cw_psia $=$ p_drop_cw ${ }^{*}$ convert $(P a, p s i a)$

\$Bookmark Cost

City\$ = 'Miami'

Call

CostAnalysis(City\$,EnergyCost,W_dot,CompCost,EvapCost,CondCost,PumpCost,HrsYr,n_yr:LC

C)

EnergyCost $=0.08[\$ / \mathrm{kW}-\mathrm{h}]$

PumpCost $=$ PumpCost_chw + PumpCost_cw

n_yr $=20$

$\mathrm{HrsYr}=1060[\mathrm{~h}]$

W_dot $=(\text { W_dot_comp }+ \text { W_dot_pump_chw }+ \text { W_dot_pump_cw })^{*} \operatorname{convert}(\mathrm{W}, \mathrm{kW})$

$\mathrm{COP}=\mathrm{Q}$ in $/ \mathrm{W}$ dot

Q_in = CoolCapacity ${ }^{*}$ convert $(\mathrm{W}, \mathrm{kW})$ 